\title{
Modeling excited states by random walks in biomolecules of increasing complexity
}

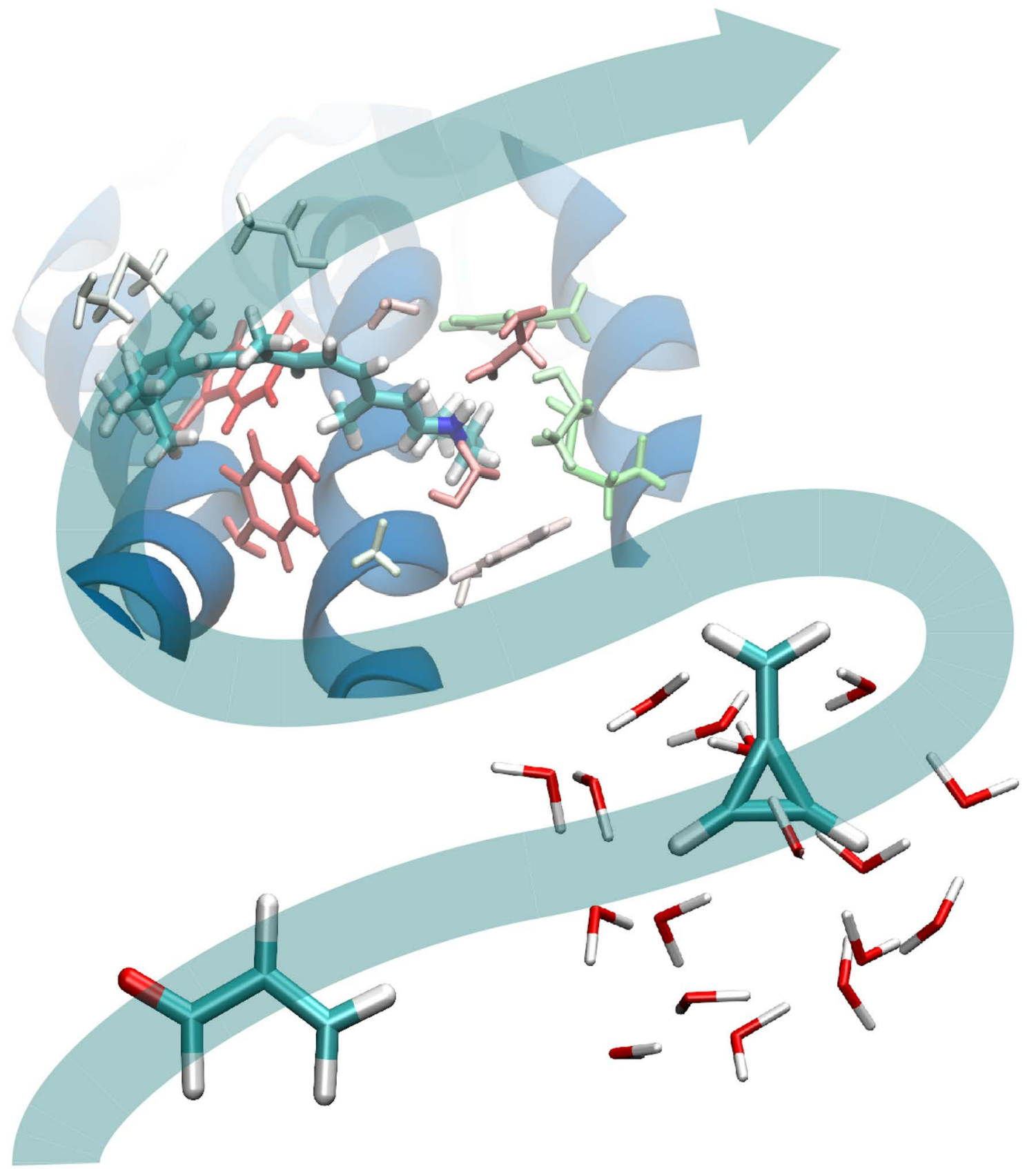


MODELING EXCITED STATES BY RANDOM WALKS IN

BIOMOLECULES OF INCREASING COMPLEXITY

Riccardo Guareschi 
Promotion committee:

Prof. dr. ir. J. W. M. Hilgenkamp University of Twente, Chairman

Prof. dr. ir. J. W. M. Hilgenkamp University of Twente, Secretary

Prof. dr. C. Filippi

University of Twente, Supervisor

Prof. dr. W. J. Briels

University of Twente

Dr. G. H. L. A. Brocks

University of Twente

Prof. dr. C. Amovilli

University of Pisa

Prof. dr. ir. G. C. Groenenboom

Radboud Universiteit Nijmegen

Dr. ir. B. Ensing

University of Amsterdam

R. Guareschi

Modeling excited states by random walks in biomolecules of increasing complexity

Ph.D. Thesis, University of Twente, Enschede

ISBN: 978-90-365-4164-0

Copyright (C) 2016 by R. Guareschi

No part of this work may be reproduced by print, photocopy or any other means without the permission in writing from the author.

DOI: $10.3990 / 1.9789036541640$

Online version: http://dx.doi.org/10.3990/1.9789036541640

Riccardo Guareschi

r.guareschi@utwente.nl

Printed by: Gildeprint Drukkerijen - www.gildeprint.nl 


\section{MODELING EXCITED STATES BY RANDOM WALKS IN BIOMOLECULES OF INCREASING COMPLEXITY}

\section{DISSERTATION}

to obtain

the degree of doctor at the University of Twente, on the authority of the rector magnificus, Prof. dr. H. Brinksma, on account of the decision of the graduation committee,

to be publicly defended on Friday $9^{\text {th }}$ of September 2016 at 12:45

by

\section{Riccardo Guareschi}

born on October 20, 1988

in Bologna, Italy 
This doctoral dissertation is approved by:

Prof. dr. C. Filippi

Promotor 



\section{Contents}

1 Introduction $\quad 1$

1.1 Predictive calculations for excited states? . . . . . . . . . . . 1

1.2 Computational spectroscopy: State of the art . . . . . . . . . 3

1.3 This thesis . . . . . . . . . . . . . . 6

2 Theoretical Methods $\quad 17$

2.1 Introduction . . . . . . . . . . . . . . . . . . 17

2.2 Quantum Mechanical Calculations . . . . . . . . . . . . . 17

2.3 Multiscale Techniques . . . . . . . . . . . . . . . . . 36

2.4 Computational Details . . . . . . . . . . . . . . . . . 41

3 Ground- and excited-state geometries of small molecules with QMC 49

3.1 Introduction . . . . . . . . . . . . . . . 50

3.2 Methods .......................... 51

3.3 Computational details . . . . . . . . . . . . . . . 54

3.4 Results . . . . . . . . . . . . . . . . . . 55

3.5 Conclusions . . . . . . . . . . . . . . . 77

4 Excited-state structures in PCM: A QMC and DFT study 85

4.1 Introduction . . . . . . . . . . . . . . . . 85

4.2 Methods . . . . . . . . . . . . . . . . . . 87

4.3 Computational details . . . . . . . . . . . . . . . . . . . 93

4.4 Results . . . . . . . . . . . . . . . . . . . . . . 94

4.5 Conclusions . . . . . . . . . . . . . . . 105

5 Introducing QMC in polarizable force fields for excited states 113

5.1 Introduction . . . . . . . . . . . . . . . . . . 114

5.2 Methods . . . . . . . . . . . . . . . . . . 115

5.3 Computational details . . . . . . . . . . . . . . . . . . . . 119

5.4 Results . . . . . . . . . . . . . . . . . . . . . . . . . . . . . . . . . . 124

5.5 Conclusions . . . . . . . . . . . . . . . . 130

6 Rhodopsin: Quantum effects from classical modeling 137

6.1 Introduction . . . . . . . . . . . . . . . . . . . 138

6.2 Results . . . . . . . . . . . . . . . . . 140 
6.3 Discussion and conclusions . . . . . . . . . . . . . . . . . . 147

6.4 Computational methods . . . . . . . . . . . . . . . . . . . 149

List of publications 156

$\begin{array}{ll}\text { Samenvatting } & 160\end{array}$

$\begin{array}{ll}\text { Acknowledgements } & 163\end{array}$ 


\section{Chapter 1}

\section{Introduction}

\subsection{Predictive calculations for excited states?}

The absorption of visible light and its conversion to other forms of energy is at the heart of some of the most fundamental processes in physics and biology. Upon light absorption, photoactive molecules (chromophores) undergo a series of transformations, triggering responses that may extend over several spatial and temporal scales. When chlorophyll molecules absorb visible light, for instance, they undergo an electronic excitation that triggers the process of photosynthesis in plants. Another familiar example is the primary event of vision: Light absorption stimulates a conformational change of the photosensitive component in the retina, which is followed by a cascade of chemical reactions culminating in the stimulation of the optical nerve. The ability of molecules to absorb light is also routinely exploited in the field of energy production in solar cells and many other applications. Deepening our physical understanding of the primary excitation processes and of the subsequent energy transfer in these photo-systems is important both from a fundamental point of view and because of existing and potential applications in biology, biotechnology and artificial photosynthetic devices.

The problem of describing photoexcitation processes is inherently quantum mechanical and falls in the realm of first-principle electronic structure computations. The exposure of a molecule to light determines an electronic transition from its ground to an excited state, where the system exhibits new chemical and physical properties, for a limited fraction of time before decaying back to the ground state. Many different factors can affect the process of electronic excitation such as the structural features of the system or the interactions between the chromophore and the other molecules surrounding it. Recent years have witnessed rapid growth in the experimental, quantitative uncovering of molecular processes induced by light absorption in complex (bio)systems. Comparison with theory is increasingly necessary to unravel the microscopic mechanisms underlying experimental observations. Despite significant progress in electronic structure theory and some noteworthy successes, however, it remains far from trivial to reliably compute the excitation properties of even relatively small photoactive molecules, let alone understand the complex 
spectral behavior of a natural bio-system.

Theoretically, predicting the optical properties of photoactive systems is difficult since it entails solving the Schrödinger equation with a consistent accuracy for ground and excited states, treating at the same time a realistically large model of the (bio)system. The theoretical approach should also allow a dynamical description of ground-state fluctuations and of photo-induced dynamical effects, namely, follow conformational changes in the excited state with a uniformly accurate description of the potential energy surface (PES) of the state of interest and of other energetically close states. A schematic representation of some of the processes which might follow light absorption is given in Fig. 1.1.

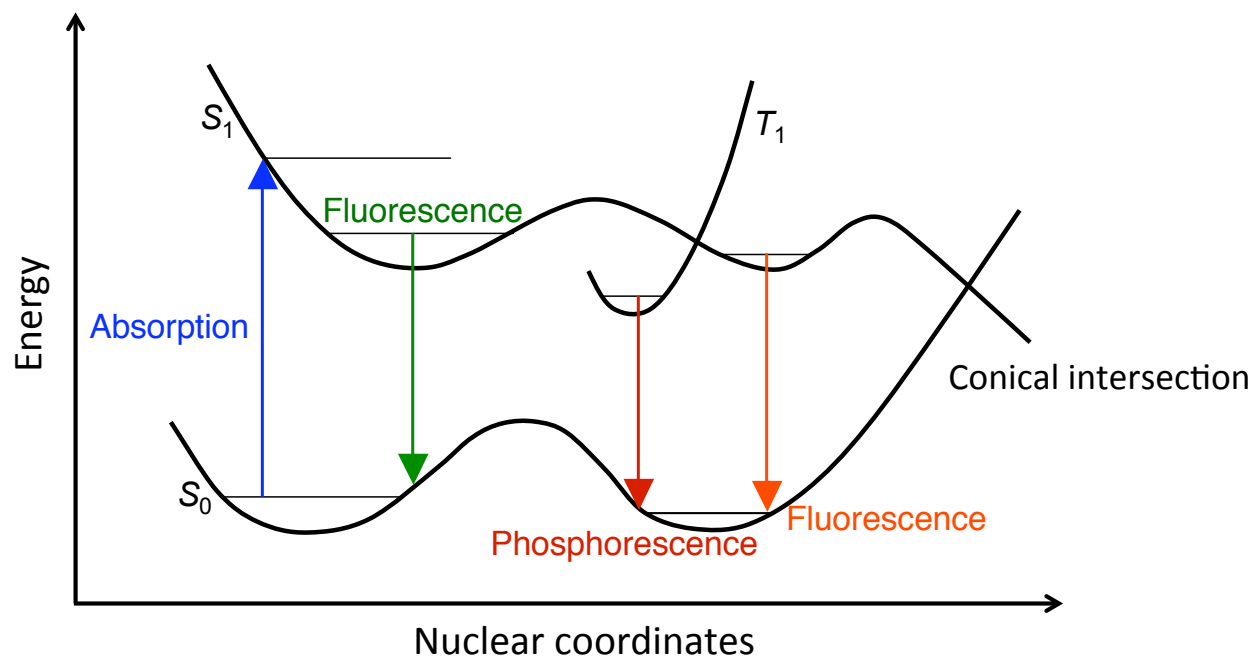

Figure 1.1: Schematic representation of the ground and an excited-state potential energy surface and of the pathways initiated by light absorption.

In most cases, ground state properties of large systems can be reliably and efficiently computed from first principles, and sufficient knowledge has also been accumulated to establish the accuracy of particular calculations. More specifically, ground-state properties can be described using density functional theory (DFT) [1] in combination with ab-initio molecular dynamics [2] to study the thermal fluctuations of the chromophore and its immediate surroundings. In biosystems, protein-chromophore interactions in the ground state can for instance be included via mixed quantum mechanics-in-molecular mechanics (QM/MM) simulations, where the photoactive site is described quantum mechanically and the interaction with the rest of the macromolecule is treated as a sea of point charges using an atomic force field $[3,4]$. On the other hand, it is generally rather difficult to reliably compute excitation properties and there are often problems with the approaches employed in the study of photoactive molecules, especially far away from the Franck-Condon region. Timedependent (TD)DFT [5] has become the standard tool for electronic structure calculations of excited states when applicable, particularly when other methods prove to be too expensive. However, known shortcomings of approximate TDDFT sometimes 
severely affect its accuracy; in particular, the domain of applicability of standard TDDFT is seriously restricted when it comes to photochemical reactions [6]. The border defining what is a conservative "safe use" of TDDFT continues to expand with the development of new functionals and techniques [7-10]. Yet, at the moment, the theoretical vacuum created by the limitations of TDDFT has been filled by traditional quantum chemical methods despite their less favorable scaling with system size, which often imposes compromises on accuracy in the case of large photosensitive molecules. Moreover, as already mentioned, the chromophore interacts with its environment, which can be as simple as an homogeneous solution or, at the other extreme, very complex with specific and directional interactions as in the case of a chromophore embedded in a protein. In either case, the study of light absorption must also account for these interactions, whose description is instead often oversimplified and kept unchanged in the photoexcitation process using for instance a static point-charge approach.

Since currently available computational techniques have limited applicability, progress in computational spectroscopy hinges on the ability to develop new computational tools for excited states that meet the many requirements listed above as well as a reliable set of reference data to assess the performance of existing and newly proposed approaches. In this work, we present our contributions in these directions, namely, the development of novel multi-scale excited-state approaches in the framework of accurate quantum Monte Carlo (QMC) methods [11-13], the construction of robust reference data for molecular excited-state structures of systems of increasing complexity (from prototypical isolated to solvated chromophores), and the analysis of the nature of polarization and the theoretical schemes needed to describe it in the challenging case of the photoactive protein rhodopsin.

\subsection{Computational spectroscopy: State of the art}

In the development of theoretical methods for excited states, the focus has primarily been on the evaluation of vertical excitation energies of molecular systems in the gas phase. Thanks to the availability of various (experimental and theoretical) routes to obtain reliable ground-state geometries, one can then investigate the performance of an approach in characterizing the excited-state potential energy surface in the FranckCondon region, being confident that errors coming from the estimate of the groundstate structure will only be minor. Moreover, a gas-phase study with the suppression of external interactions allows the clear identification of the intrinsic ability of a certain quantum method to predict these excitation energies. In the literature, one can find a large number of studies and benchmark work that follow this strategy [14-18].

Nonetheless, if one is interested in photochemistry, it is important to move beyond the primary process of light absorption, with fluorescence being the next important process an excited-state method should accurately describe. In this event, a molecule emits a photon from the minimum of its excited-state PES (see Figure 1.1) and the energy of the photon is estimated as the vertical gap between this point and the PES of the ground state. We are now departing from the Franck-Condon region 
and the quality of the results clearly depends on the ability of a given method to provide accurate interatomic excited-state forces to correctly characterize the excitedstate PES in the neighborhood of this minimum. The many available methods typically display large differences in their performance both in terms of computational cost and accuracy of the resulting excited-state structures [19-23].

Therefore, in the study of excited states, one faces multiple difficulties: Once one has implemented geometrical energy gradients at a certain level of theory, one needs to identify an approach that can be as reference to access the quality of the obtained results. For instance, TDDFT can be readily employed to obtain excitedstate geometries also in fairly large systems but the results strongly depend on the chosen exchange-correlation functional and on the physical nature of the excitation considered with severe limitations, in particular, for charge-transfer or multiconfigurational excited states [24,25]. Finding a robust reference method is however a non-trivial task. For instance, the coupled cluster approximate singles and doubles (CC2) method is becoming quite popular in this field since it can be applied to relatively large systems (thanks to the so-called reduction-of-virtual-space scheme [26]) but it does not always represent on improvement on TDDFT as we will also show in this thesis. Multireference pertubation theories are generally more effective but the construction of the zero-order wave function is often rather tricky and the results can be very sensitive to the choice of ingredients in the calculation, especially when the perturbation correction is large. We were ourselves surprised on how difficult a small and apparently harmless acrolein molecule in its $\pi \rightarrow \pi^{*}$ state can be and how long (weeks!) we needed to play to obtain stable excitation energies and geometries with these perturbation approaches. This general scenario and our own experience has deeply convinced us of the need to further push the very different quantum Monte Carlo techniques for the computation of excited-state structures and, in the process of testing them, provide reliable reference data for the structural properties in the excited states of prototypical organic molecules.

The problems illustrated so far in the study of excited-state properties increase when one considers a molecule surrounded by an environment, which affects its molecular properties already in the ground state. The environment can for instance significantly distort the optimal gas-phase geometry or shift the relative position of the ground- and excited-state PES's, changing the wavelength of the light that is absorbed. To simulate the presence of an environment, it is necessary to determine whether it acts as a simple "spectator" in the excitation process of the chromophore, carrying only an external perturbation, or if it actively participates as it could happen in the case of charge-transfer or resonance excitations between the chromophore and the surrounding molecules. The most commonly used multiscale approaches are appropriate to describe the first eventuality but are often mindlessly employed in the study of all sorts of bio-chromophore: The system is partitioned in two regions with the chromophore treated quantum mechanically $(\mathrm{QM})$, and the rest of the environment treated with the cheaper methods of the classical molecular mechanics (MM), where a set of static point charges optimized within a force field are placed on the positions of the atoms of the environment. Although very successful for the treatment of ground states, there is mounting evidence from work of our and other groups [27-32] 
that such a scheme is not suitable in the study of the absorption properties of various photobiological systems: The use of frozen embedding relies on the implicit assumption that the photoexcitation does not perturb the surroundings of the active region, which is surely far from realisitc in many common situations where the electronic distributions of the states involved in the electronic transition are significantly different. If various studies have finally started to discuss the optimal combined choice of approaches to describe the chomrophore-environment coupling in computing absorption properties, very little is known on the structural excited-state response beyond the gas phase. A TDDFT study of optimal excited-state geometries of prototypical molecules in the simplest model for a solvent, namely, the polarizable continuum model (PCM), has recently appeared, totally lacking however any reference data to assess the correctness of the results [33]. This is a gap we try to fill with this thesis. Overall, the excited-state structural relaxation of a chromophore and its dependence on the model chosen to couple it to its surroundings is an issue still largely unexplored in computational spectroscopy. As we discuss later, our findings on rhodopsin absorption open several questions on how this coupling should be described away from the Franck-Condon region.

If we remain within a computationally affordable quantum-in-classical partition scheme, the embedding model can for instance be improved by employing polarizable force fields (QM/MMpol) [34-37], which introduce classical induced dipoles to take into account mutual polarization effects between the quantum and the classical systems in the ground state and in response to the electronic excitation, thus going beyond the frozen-environment approximation. In the state-specific formulation, one induces the classical dipoles to minimize the polarization energy given by the classical part with respect to a certain electronic density of the quantum region, either the one of the ground or the excited state. Therefore, there is not a unique external potential for all the electronic states but it is possible to optimize the interactions with a specific state. In the case of the simulation of light absorption, differential electrostatic effects are fully included in computing the excitation energies with such a scheme.

Such a formulation is clearly superior to point-charge embedding but only captures the electrostatic response of the environment in equilibrium with the chromophore and still misses "resonance" effects, which would be recoverd in a quantum formulation including coupled excitations chromophore-chromophore/environmentenvironment. Perhaps naïfly, we were believing these contributions to be generally relatively small, especially in a system like rhodopsin where the excitation is characterized by large charge variations on the chromophore and the environment consists of amino acids featuring energetically much higher excitations. We were mistaken and we demonstrate so in this thesis exploiting a feature of polarizable classical embedding that is not well known and sometimes poorly understood in literature: One can use a polarizable classical set of dipoles (the same holds for the PCM) to mimic this type of interactions. In a linear-response formulation, the dipoles respond not to the change in electronic density of the chromophore but to the transition density between the states involved in the excitation, generating a potential which effectively couples the states on the chromphore and therefore introduces effects which are truly 
non-classical. This approach recovers the resonant polarization effects of the environment coupled to the excitation of the chromophore and does not account for electrostatic relaxation effects, which are instead captured in the state-specific approach. Importantly, it signals us the need of a treatment with goes beyond a description in terms of the product of wave functions of the chromophore and the environment. Finally, it also allow us to identify the amino acids which respond electrostatically and/or resonantly, which, in rhodopsin, form a relatively large shell surrounding the chromophores, too large for a purely quantum treatment using a high-level correlated approach. In a real system, electrostatic and resonance effects of course coexist, concurring in the collective effects observed at the experimental level, and should be described simultaneously in a simulation. A polarizable embedding in a state-specific and a linear-response fashion allows us to recover them separately and to account for them in an additive manner in the computation of the excitation energy.

If a complete quantum treatment is not possible for the system under study, it remains an open question how to handle the excited-state structural optimization of a chromophore in a complex environment. Should one use a state-specific or a linear-response approach if we maintain a QM/MMpol description of the system? Should these corrections contribute additively to the interatomic excited-state forces? A recent study [38] using TDDFT in a PCM solvent has shown for a set of small molecules that the excited-state geometries obtained in the state-specific and linearresponse schemes are rather similar. This finding is surprising and possibly due to the particular choice of systems or to the TDDFT approach: A transition characterized by a small oscillator strength and a large change in molecular dipole moment will give a small correction in linear response and a significant differential polarization contribution to the excitation energy. Therefore, one would expect the optimization to give rather different excited-state structures in the two formulations. All of this clearly shows that the issue is very open and requires more scrutiny with further benchmark studies on prototypical systems as well as more problematic chromophores, focusing not on vertical excitation energies but on more difficult points of the excited-state PES of a molecule such as for instance near a conical intersection region. In this thesis, we have developed the needed tools in the framework of quantum Monte Carlo methods which, together with the insight gained on the prototypical photoactive protein rhodopsin, will be instrumental in future studies to further explore the role of the environment description on the structural relaxation in the excited state.

\subsection{This thesis}

In this thesis, we will deepen the discussion on the issues presented in the previous Section, focusing on the results obtained with quantum Monte Carlo on the excitedstate geometry optimizations of prototypical molecules (in comparison with TDDFT and other wave function-based methods) first in the gas phase and then in a PCM solvent. We will subsequently illustrate how to model the environment more realistically through charges and induced dipoles to describe light absorption of organic chromophores and of a complex photoactive protein, comparing static and polariz- 
able embedding conditions.

In Chapter 2, we present the different theoretical methods we use and also further develop in this work. We describe the basic principles behind wave-function based approaches, focusing on the continuum quantum Monte Carlo methods and on the multi-reference perturbation techniques that are most commonly used for the calculation of excited-state properties of (bio)molecular systems. In addition, we give a brief overview of density functional theory and its extension to the treatment of excited states, namely, time-dependent density functional theory.

In Chapter 2, we also describe the general features of different multiscale approaches. We begin with the polarizable continuum model (PCM) $[39,40]$, which provides a simplified description of the environment as a classical dielectric around a molecule and which we here combine with QMC for the structural optimization of solvated molecules in the excited state. The presence of this polarizable continuum medium is simulated through a distribution of point charges placed on the surface of a cavity formed by a set of interlocking spheres centered on the atoms. Their magnitude is determined by the dielectric constant of the medium and by the charge density of the molecule enclosed in the cavity. Despite its simplicity, this model is very useful to represent the bulk mean effects of a solvent but cannot account for specific interactions between a molecule and its surroundings.

In coupling quantum mechanics to treat a photoactive system and classical mechanics to represent the environment, one can attempt to achieve a more realistic description, preserving the discrete nature of the environment in a so-called quantummechanics-in-molecular-mechanics approach (QM/MM). This replaces the atoms of the embedding system with point charges which perturb the chromophore via their external electric field $[3,4,41]$. This embedding scheme is widely used in computational chemistry and can be very effective in recovering ground-state effects but, in the study of excited states, is sometimes rather inaccurate since the perturbation induced by the external field is the same for all states of interest. The application of this approach to excited states can be improved by including also atomic polarizable dipoles [34-36]. This allows a more flexible description of the quantum-classical interactions and the use of state-specific dipoles to account for the relaxation of the environment upon excitation of the chromophore often leads to significant improvements with respect to static QM/MM embedding. The nature of the effects captured by this polarizable scheme will be the focus of the last two Chapters.

In Chapter 3, we begin with a gas-phase study to assess the quality of the excitedstate geometries computed with several electronic structure methods for small prototypical chromophores. This comparison serves a double purpose. First, we want to investigate the performance of the simplest flavor of QMC (variational Monte Carlo) in optimizing excited-state structures and its robustness with respect to the various ingredients entering the trial wave function. Thanks to recent developments, the efficient computation of energy gradients in QMC has become possible [42,43] but relatively few applications have appeared so far in the literature. QMC has in fact been extensively employed in the study of ground-state properties and, more recently, our group has pushed its development and application to the computation of excitation energies of prototypical chromophores [30,32,44-48]. Its use for the optimization of 
excited-state geometries is new and a thorough comparison with other popular correlated approaches is therefore timely. In addition, with this study, we want to offer a reliable benchmarking set of optimal excited-state geometries which can be used as reference in the development of novel DFT functionals targeting excited states. With relatively few exceptions [21-23,49], the benchmarking of TDDFT has so far focused on the calculations of vertical excitation energies, also in part due to the lack of good comparison data for structural parameters.

In this Chapter, we confirm the reliability of QMC in the calculation of molecular gradients, showing that the ground- and excited-state QMC geometries agree very well with those computed with different perturbation approaches. Furthermore, the QMC estimates are very robust as compared to these perturbation techniques, which are sometimes rather sensitive to the computational parameters entering the calculation. This good performance combined with the favorable scaling with system size a mere $\mathcal{O}\left(N^{4}\right)$ with the number of electrons $N$ - renders this method particularly appealing for the structural optimization of larger molecules, especially in cases where other methods are inaccurate or not reliable. Finally, our calculations well illustrate the inadequacy of TDDFT in predicting structural changes in the excited state if the optimization brings us far from the Franck-Condon region. The spread in the TDDFT structures obtained with the different exchange-correlation functionals is also rather large and does not reveal any clear relation between a particular family of functionals and the quality of the corresponding optimized structures as illustrated in Figure 1.2.

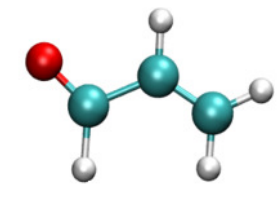

acrolein

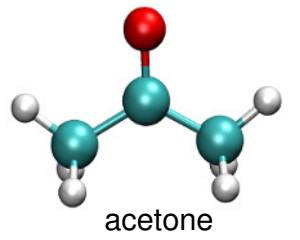

acetone

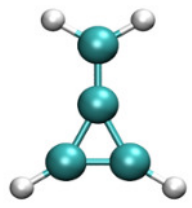

MCP

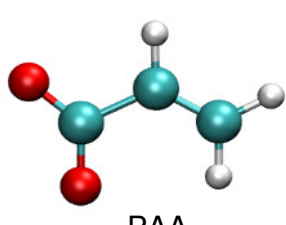

PAA

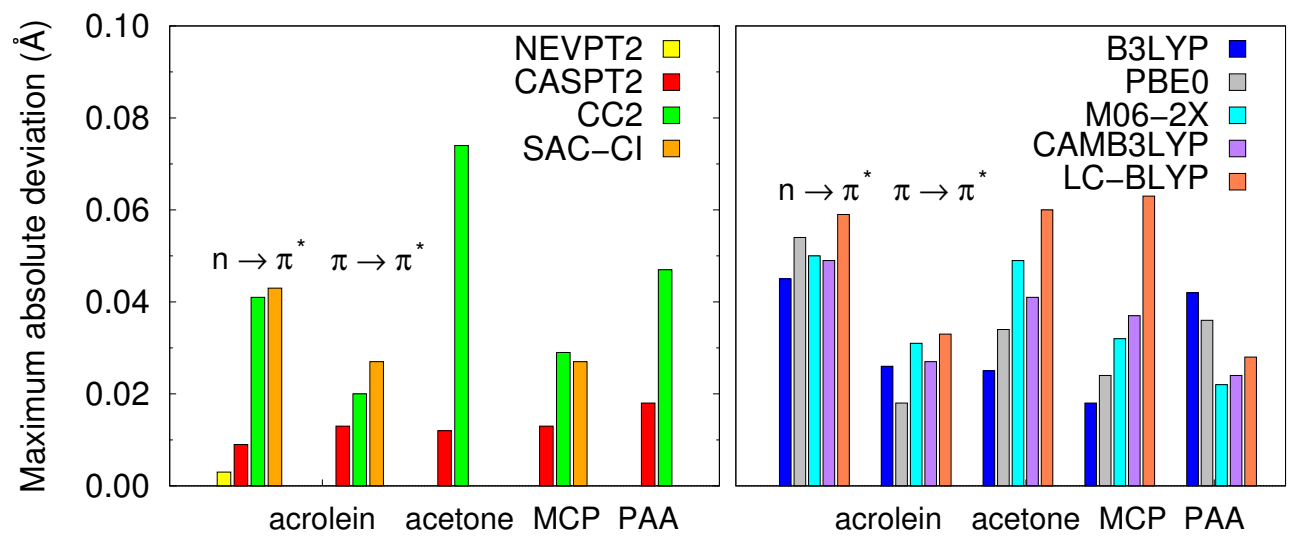

Figure 1.2: Comparison of the maximum absolute deviations $(\AA)$ of the optimal excited-state geometries of the molecules depicted above, computed with wavefunction-based methods (left) and TDDFT (right) with respect to the optimal geometries optimized within QMC.

Strong of the findings of Chapter 3, we combine for the first time QMC with 
the polarizable continuum model to perform excited-state geometry optimization in solution in Chapter 4. In our implementation, the external field of the continuum medium, representing here the bulk of a water solution, is determined selfconsistently with the molecular wave function during the QMC optimization of the solute geometry. The spheres that define the cavity follow the atoms of the solute during the optimization. Our interest here is to develop a computational tool in the framework of QMC methods, which will allow us to gain a "quick" qualitative understanding in future applications of how a photoexcited chomophore responds structurally to the presence of a medium. Furthermore, no benchmark data exist to assess the quality of the response of the excited-state structures computed with TDDFT to the presence of an environment, not even modeled with the simplest PCM model. In this Chapter, we show that all investigated TDDFT functionals overestimate the excited-state geometrical response to the presence of a solvent, sometimes giving bond variations opposite in trend to QMC.

In Chapter 5, we abandon the topic of geometry optimization and focus on modeling absorption properties in a more realistic discrete representation of a responsive environment. This diversion was prompted by the difficulties we encountered in recent years to reproduce the experimental absorption maxima of various photoactive proteins when employing a static QM/MM point-charge description. While intended as a first step in the study of structural relaxation in photoexcited rhodopsin, this project has become the center of my thesis and will be the focus of the last two Chapters. In Chapter 5, we develop a hybrid scheme of QMC in classical charges and polarizable dipoles (QMC/MMpol) and investigate the ability of such a multiscale method to describe the excitations of two small representative molecules, namely, methylenecyclopropene and $s$-trans acrolein in water solution. To do so, we move in two directions: On the one hand, in the same spirit of the previous Chapters, we employ a variety of electronic structure techniques to treat the quantum solute. On the other hand, we analyze the dependence of the results on the different formulations chosen to model the environment. In particular, we show that static point-charge embedding does not recover important physical effects present in the solute-solvent coupling. This embedding can be effectively improved if the dipoles are self-consistently brought to equilibrium with the electronic density of the different states. In this framework, the classical part perturbs the quantum subsystem and in turn responds to the variations of its electronic distribution upon photoexcitation of the quantum part. Mathematically, this means that the Schrödinger equation for the quantum subsystem is coupled with the classical equation that determines the external potential and one has to solve the two problems iteratively, as schematized in Figure 1.3.

Using such an embedding method, we can investigate different polarization conditions to identify the nature of the solute-solvent coupling. A first option is to optimize the dipoles with respect to the ground-state solute density, and then compute the excitation energies in the field of this improved, yet frozen, environment. A second possibility is to determine two different external potentials by generating two sets of dipoles, separately equilibrated with the two states involved in the electronic transition, thus recovering the response of the environment to the excitation. The compar- 


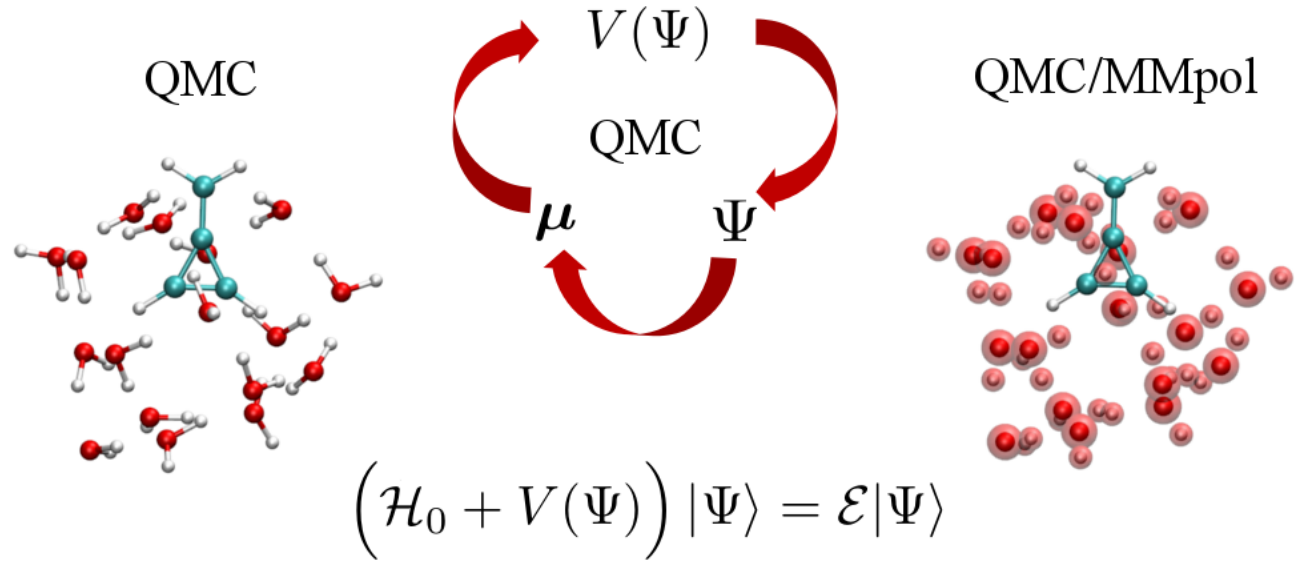

Figure 1.3: Schematic representation of the self-consistent cycle between the solute wave function and the external potential. $\mathcal{H}_{0}$ represents the Hamiltonian of the isolated molecule in the field of the static point charges.

ison of these results with supermolecular calculations, where both solute and solvent are treated quantum mechanically, reveals that state-specific embedding produces superior results than the use of a frozen environment (point charges or ground-state optimized dipoles), in particular, when the chromophore exhibits a large variation of the molecular dipole moment between ground and excited state and the solutesolvent coupling is dominated by electrostatic effects.

With this superior tool to treat polarization when a point-charge description of the environment is not adequate, in Chapter 6, we move to the difficult case of light absorption in rhodopsin. Rhodopsin is the opsin responsible for dim-light vision in humans and belongs to the family of visual opsins [50-53]. Most opsins share the same photosentive component, namely, the 11-cis retinal, which is covalently linked to the backbone of the protein via an imine bond with the adjacent lysine residue, thus creating the retinal protonated Schiff base (RPSB) chromophore (see Figure 1.4).

Even though they share the same chromophore, the proteins belonging to the opsin family absorb over a wide range of frequencies. In humans, for instance, rhodopsin absorbs at $500 \mathrm{~nm}$, while the other photoreceptors for color vision absorb around $425 \mathrm{~nm}$ (blue), $530 \mathrm{~nm}$ (green), and $560 \mathrm{~nm}$ (red). For other animals, the spectral coverage can span a much wider range, from 300 to $700 \mathrm{~nm}$ [54]. This effect, called opsin shift, is the result of many specific interactions between the chromophore and the protein environment, which have been intensively studied both experimentally and theoretically but whose nature is still to be clarified [55]. Qualitatively, two opposite effects are present. As shown in Fig. 1.4, the negative counterion placed in the proximity of the positive nitrogen of RPSB stabilizes its ground state more the excited state, thus inducing a blue-shift of the excitation with respect to the gas phase. The excited state is in fact characterized by charge migration from the $\beta$-ionone 


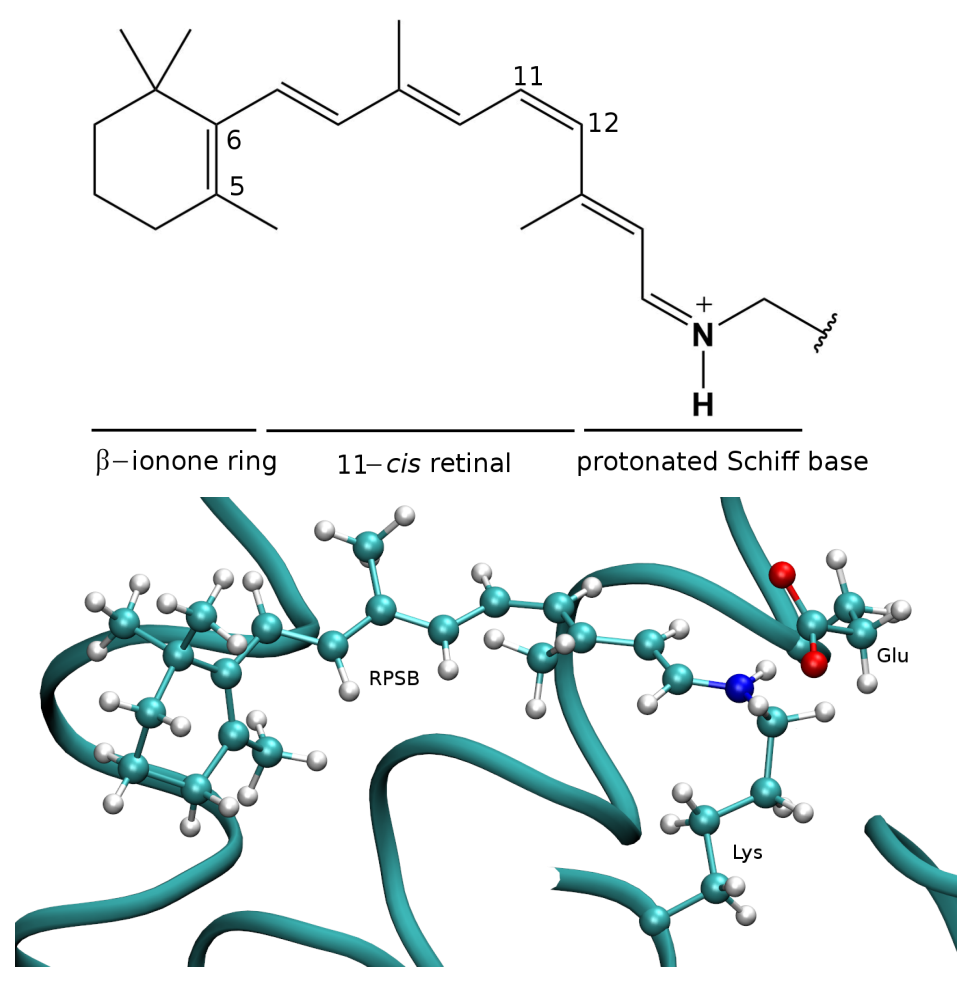

Figure 1.4: Retinal protonated Schiff base (RPSB) chromophore with atomic labels (top) and RPSB in its protein environment (bottom). We also show the position of the counterion glutamic acid.

ring towards the nitrogen atom, which is opposed by the repulsive interaction with the electronic density of the counterion. This strong blue-shift is modulated by the rest of the environment and partially quenched. Although such a picture is widely accepted, the quantification of the different effects is very challenging leading to controversial results and very different estimates. In this Chapter, we want to understand the origin of these effects and answer some fundamental questions: Is electrostatic coupling dominant as implicitly assumed when adopting a multiscale quantum-in-classical description? Are there more suitable quantum interactions we are neglecting? While most rhodopsin studies in the literature have employed static point charges, we answer here these questions using a polarizable embedding QM/MMpol scheme. This powerful and flexible approach allows us to quantify the role in color tuning of the different amino acids in the binding pocket of RPSB as well as the nature of their interactions: The chromophore and the protein can be made to interact following different schemes which recover different physical effects and can also also mimic a coupling which is not purely electrostatic and, therefore, in principle not amenable to a classical description.

For several frames extracted from a QM/MM molecular dynamics to account for temperature effects, we compute the excitation energies in the field of dipoles polarized to the ground state or in a state-specific fashion. Furthermore, we induce the classical dipoles within linear-response theory as responding to the RPSB transition 
density associated to the electronic transition. The excitation energies obtained with different quantum methods consistently show that the use of point charges is responsible for a strong blue-shift, which is further enhanced when a frozen environment of dipoles equilibrated with the ground state of RPSB is used, as a consequence of the further stabilization of the ground state with respect to the excited state. As expected given the large density difference between the ground and the excited state of RPSB, accounting for differential polarization effects inverts this trend, yielding a red-shift. Nevertheless, the inclusion of electrostatic differential polarization does not account for the complete description of the chromophore-protein coupling. The use of dipoles induced in the linear-response regime in resonance with the transition dipole moment of RPSB has also a large impact on the excitation energies, indicating that non-classical, resonant interactions between RPSB and several amino acids in its environment are important. When this effect is accounted for together with the electrostatic response of the environment to the excitation, we obtain excitation energies in good agreement with the experimental absorption maximum of rhodopsin. 


\section{Bibliography}

[1] R. M. Dreizler and E. K. U. Gross, Density Functional Theory, An Approach to the Quantum Many-Body Problem (Springer-Verlag: New York, 2009).

[2] D. Marx and J. Hutter, Ab initio molecular dynamics: basic theory and advanced methods (Cambridge University Press, 2009).

[3] H. M. Senn and W. Thiel, Angew. Chem. Int. Ed. 48, 1198 (2009).

[4] G. Seabra, J. Swails, and A. Roitberg, in Multi-Scale Quantum Models for Biocatalysis, Vol. 7 of Challenges and Advances in Computational Chemistry and Physics, edited by D. York and T.-S. Lee (Springer: New York, 2009), pp. 3-20.

[5] C. A. Ullrich, Time-dependent density-functional theory: concepts and applications (OUP Oxford, 2011).

[6] M. Casida and M. Huix-Rotllant, Annu. Rev. Phys. Chem. 63, 287 (2012).

[7] B. Kaduk and T. Van Voorhis, J. Chem. Phys. 133, 061102 (2010).

[8] S. L. Li, A. V. Marenich, X. Xu, and D. G. Truhlar, J. Phys. Chem. Lett. 5, 322 (2014).

[9] M. Huix-Rotllant, M. Filatov, S. Gozem, I. Schapiro, M. Olivucci, and N. Ferré, J. Chem. Theory and Comput. 9, 3917 (2013).

[10] E. Fromager, S. Knecht, and H. J. A. Jensen, J. Chem. Phys. 138, 084101 (2013).

[11] W. M. C. Foulkes, L. Mitas, R. J. Needs, and G. Rajagopal, Rev. Mod. Phys. 73, 33 (2001).

[12] J. Kolorenc and L. Mitas, Rep. Prog. Phys. 74, 026502 (2011).

[13] B. M. Austin, D. Y. Zubarev, and W. A. Lester, Chem. Rev. 112, 263 (2012).

[14] C. Hättig and F. Weigend, J. Chem. Phys. 113, (2000).

[15] M. J. G. Peach, P. Benfield, T. Helgaker, and D. J. Tozer, J. Chem. Phys. 128, 044118 (2008). 
[16] M. Schreiber, M. R. Silva-Junior, S. P. A. Sauer, and W. Thiel, J. Chem. Phys. 128, 134110 (2008).

[17] D. Jacquemin, V. Wathelet, E. A. Perpète, and C. Adamo, J. Chem. Theory Comput. 5, 2420 (2009).

[18] M. R. Silva-Junior and W. Thiel, J. Chem. Theory Comput. 6, 1546 (2010).

[19] C. A. Guido, D. Jacquemin, C. Adamo, and B. Mennucci, J. Phys. Chem. A 114, 13402 (2010).

[20] R. Cammi, R. Fukuda, M. Ehara, and H. Nakatsuij, J. Chem. Phys. 133, 024104 (2010).

[21] F. Furche and R. Ahlrichs, J. Chem. Phys. 117, 7433 (2002).

[22] D. Bousquet, R. Fukuda, P. Maitarad, D. Jacquemin, A. Ciofini, C. Adamo, and M. Ehara, J. Chem. Theory Comput. 9, 2368 (2013).

[23] A. D. Laurent and D. Jacquemin, Int. J. Quantum Chem. 113, 2019 (2013).

[24] A. Dreuw, J. L. Weisman, and M. Head-Gordon, J. Chem. Phys. 119, 2943 (2003).

[25] M. E. Casida, J. Chem. Phys. 122, 054111 (2005).

[26] R. Send, V. R. I. Kaila, and D. Sundholm, J. Chem. Phys. 134, 214114 (2011).

[27] M. Wanko, M. Hoffmann, T. Frauenheim, and M. Elstner, J. Phys. Chem. B 112, 11462 (2008).

[28] M. Wanko, M. Hoffmann, J. Frähmcke, T. Frauenheim, and M. Elstner, J. Phys. Chem. B 112, 11468 (2008).

[29] C. Filippi, F. Buda, L. Guidoni, and A. Sinicropi, J. Chem. Theory Comput. 8, $112(2012)$

[30] O. Valsson, P. Campomanes, I. Tavernelli, U. Rothlisberger, and C. Filippi, J. Chem. Theory Comput. 9, 2441 (2013).

[31] V. R. I. Kaila, R. Send, and D. Sundholm, Phys. Chem. Chem. Phys. 15, 4491 (2013).

[32] C. Daday, C. Curutchet, A. Sinicropi, B. Mennucci, and C. Filippi, J. Chem. Theory Comput. 11, 4825 (2015).

[33] C. A. Guido, S. Knecht, J. Kongsted, and B. Mennucci, J. Chem. Theory Comput. 9, 2209 (2013).

[34] M. A. Thompson, J. Phys. Chem. 100, 14492 (1996). 
[35] C. Curutchet, A. Muñoz-Losa, S. Monti, J. Kongsted, G. D. Scholes, and B. Mennucci, J. Chem. Theory Comput. 5, 1838 (2009).

[36] J. M. Olsen, K. Aidas, and J. Kongsted, J. Chem. Theory Comput. 6, 3721 (2010).

[37] L. V. Slipchenko, J. Phys. Chem. A 114, 8824 (2010).

[38] S. Chibani, A. D. Laurent, A. Blondel, B. Mennucci, and D. Jacquemin, J. Chem. Theory Comput. 10, 1848 (2014).

[39] J. Tomasi and M. Persico, Chem. Rev. 94, 2027 (1994).

[40] J. Tomasi, B. Mennucci, and R. Cammi, Chem. Rev. 105, 2999 (2005).

[41] H. Lin and D. Truhlar, Theor. Chem. Acc. 117, 185 (2007).

[42] S. Sorella and L. Capriotti, J. Chem. Phys. 133, 234111 (2010).

[43] S. Moroni, S. Saccani, and C. Filippi, J. Chem. Theory Comput. 10, 4823 (2014).

[44] C. Filippi, M. Zaccheddu, and F. Buda, J. Chem. Theory Comput. 5, 2074 (2009).

[45] O. Valsson and C. Filippi, J. Chem. Theory Comput. 6, 1275 (2010).

[46] R. Send, O. Valsson, and C. Filippi, J. Chem. Theory Comput. 7, 444 (2011).

[47] C. Filippi, F. Buda, L. Guidoni, and A. Sinicropi, J. Chem. Theory Comput. 8, 112 (2012).

[48] O. Valsson, C. Angeli, and C. Filippi, Phys. Chem. Chem. Phys. 14, 11015 (2012).

[49] R. Send, O. Valsson, and C. Filippi, J. Chem. Theory Comput. 7, 444 (2011).

[50] M. A. van der Horst and K. J. Hellingwerf, Acc. Chem. Res. 37, 13 (2004).

[51] M. B. Nielsen, Chem. Soc. Rev. 38, 913 (2009).

[52] H. W. Choe, J. H. Park, Y. J. Kim, and O. P. Ernst, Neuropharmacology 60, 52 (2011).

[53] Biochim. Biophys. Acta 1837, 710 (2014).

[54] O. P. Ernst, D. T. Lodowski, M. Elstner, P. Hegemann, L. S. Brown, and H. Kandori, Chem. Rev. 114, 126 (2014).

[55] K. Katayama, S. Sekharan, and Y. Sudo, in Optogenetics: Light-Sensing Proteins and Their Applications, edited by $\mathrm{H}$. Yawo, H. Kandori, and A. Koizumi (Springer Japan, 2015), pp. 89-107. 


\section{Chapter 2}

\section{Theoretical Methods}

\subsection{Introduction}

In this thesis, we employ a wide range of electronic structure methods to study the ground and the excited states of systems of variable complexity, ranging from isolated prototypical chromophores to light-sensitive molecules in realistic environments such as solutions and proteins. While small isolated molecules can be treated at a high level of theory, large embedded chromophores in a medium impose the use of computationally cheaper and more approximate approaches. Here, we will focus on multiscale approaches combining a quantum treatment of the molecule(s) primarily responsible for the response to light with a classical description of the environment. The main issues explored relate to how to define and develop the best computational strategy to combine accuracy and efficiency in such a multiscale calculation of excited states. To this aim, we will compare the results obtained using a variety of quantum methods for the photoactive site in combination with different lower-level descriptions of the environment. Whenever feasible, we will assess the quality of the multiscale results against supermolecular reference calculations, where the full system is treated quantum mechanically.

In this chapter, we briefly describe the theoretical methods employed in this thesis. In particular, we will summarize the formalism of multi-configurational selfconsistent field (MCSCF) as well as its perturbative extensions, the quantum Monte Carlo (QMC) method, density functional theory (DFT), and time-dependent DFT (TDDFT). Likewise, we will explain how to couple these quantum methods with a classical description of the environment via a quantum mechanics-in-molecular mechanics (QM/MM) scheme and its polarizable variant (QM/MMpol).

\subsection{Quantum Mechanical Calculations}

All our calculations are carried out in the Born-Oppenheimer approximation $[1,2]$ and neglecting relativistic effects so that a system of $N$ interacting electrons is de- 
scribed by the Hamiltonian (in atomic units):

$$
\mathcal{H}=-\frac{1}{2} \sum_{i=1}^{N} \nabla_{i}^{2}+\sum_{i=1}^{N} v_{\text {ext }}\left(\mathbf{r}_{i}\right)+\sum_{i<j}^{N} \frac{1}{\left|\mathbf{r}_{i}-\mathbf{r}_{j}\right|},
$$

The external potential, $v_{\text {ext }}\left(\mathbf{r}_{i}\right)$, is either the bare electron-ion Coulomb potential $-Z / r$ where $Z$ is the charge of the ion, or a pseudopotential describing the ion plus the core electrons which have been eliminated from the calculation. In the following, we denote with $\mathbf{x}=(\mathbf{r}, \sigma)$ the 3 spatial and 1 spin coordinates of one electron where $\sigma= \pm 1$.

\subsubsection{Traditional Quantum Chemistry Methods}

The simplest way to approximate the wave function of a multi-electron system, $\Psi$, is to express it as a single Slater determinant built from a set of variationally optimized single-particle spin orbitals, $\left\{\Phi_{i}\right\}$. In this approach, known as the Hartree-Fock approximation (HF) [1,2], the wave function is written as

$$
\Psi_{\mathrm{HF}}\left(\mathbf{x}_{1}, \ldots, \mathbf{x}_{N}\right)=\frac{1}{\sqrt{N !}}\left|\begin{array}{cccc}
\Phi_{1}\left(\mathbf{x}_{1}\right) & \Phi_{1}\left(\mathbf{x}_{2}\right) & \ldots & \Phi_{1}\left(\mathbf{x}_{N}\right) \\
\Phi_{2}\left(\mathbf{x}_{1}\right) & \Phi_{2}\left(\mathbf{x}_{2}\right) & \ldots & \Phi_{2}\left(\mathbf{x}_{N}\right) \\
\vdots & \vdots & \vdots & \vdots \\
\Phi_{N}\left(\mathbf{x}_{1}\right) & \Phi_{N}\left(\mathbf{x}_{2}\right) & \ldots & \Phi_{N}\left(\mathbf{x}_{N}\right)
\end{array}\right|
$$

and the single-particle spin orbitals minimize the expectation value of the interacting Hamiltonian. By expressing the spin-orbitals as the product of a spatial and a spin function, $\Phi_{i}(\mathbf{x})=\phi_{i}(\mathbf{r}) \chi_{s_{i}}(\sigma)$, the minimization of the energy associated to the Hartree-Fock determinant leads to the generalized Fock equation for the spatial orthonormal orbitals

$$
f(i)\left|\phi_{i}\right\rangle=\sum_{j=1}^{N} \epsilon_{i j}\left|\phi_{j}\right\rangle,
$$

where the sum runs over the occupied orbitals and $f$ is the mono-electronic Fock operator,

$$
f(i)=-\frac{1}{2} \nabla_{i}^{2}+v_{\text {ext }}(i)+v_{\mathrm{HF}}(i) .
$$

The Hartree-Fock potential, $v_{\mathrm{HF}}$, represents an effective potential experienced by one electron due to the presence of the other electrons. Therefore, the essence of the Hartree-Fock approximation is to replace the interacting many-electron problem with a set of independent particles in a mean field. The Hartree-Fock potential can be written as the sum of two terms, that is, the local Coulomb electrostatic potential, $v_{\text {Coul }}$, and the non-local exchange potential, $v_{\mathrm{x}}^{\mathrm{HF}}$ :

$$
\begin{aligned}
v_{\text {Coul }}(\mathbf{r}) & =\sum_{j=1}^{N} \int \frac{\left|\phi_{j}\left(\mathbf{r}^{\prime}\right)\right|^{2}}{\left|\mathbf{r}-\mathbf{r}^{\prime}\right|} d \mathbf{r}^{\prime} \\
\left(v_{\mathrm{x}}^{\mathrm{HF}} \phi_{i}\right)(\mathbf{r}) & =-\sum_{j=1}^{N} \delta_{s_{i}, s_{j}}\left[\int \frac{\phi_{j}^{*}\left(\mathbf{r}^{\prime}\right) \phi_{i}\left(\mathbf{r}^{\prime}\right)}{\left|\mathbf{r}-\mathbf{r}^{\prime}\right|} d \mathbf{r}^{\prime}\right] \phi_{j}(\mathbf{r}) .
\end{aligned}
$$


Since the Hartree-Fock wave function, its energy, and the Fock operator are invariant under a unitary transformation of the occupied orbitals, there is not a unique set of orbitals satisfying Equation (2.2): A unitary transformation of the orbitals which are solution of this equation produces a new set of orbitals satisfying the same equation. It is however convenient to choose the unitary transformation that diagonalizes the matrix with elements $\epsilon_{i j}$. The orbitals giving a diagonal representation of the Fock operator are called canonical orbitals and the diagonal elements $\epsilon_{i}$ can be interpreted through Koopmans' theorem with the physically meaningful ionization energies of the system in the additional approximation that the orbitals do not relax when an electron is removed or added to the system [3]

The solution of a HF problem entails starting from a set of guess orbitals, determining the HF potential for these orbitals, and repeating the procedure until selfconsistency. The molecular orbitals are expanded as a linear combination of atomic orbitals (LCAO) centered on the nuclear positions:

$$
\phi_{i}(\mathbf{r})=\sum_{\mu}^{\text {nuclei }} \sum_{j} a_{j i}^{\mu} \eta_{j \mu}\left(\mathbf{r}-\mathbf{r}_{\mu}\right),
$$

where $\mathbf{r}_{\mu}$ denotes the position of a nucleus, and the minimization is performed with respect to the LCAO coefficients, $a_{j i}^{\mu}$. In most quantum chemistry codes, a Gaussian atomic basis is used:

$$
\eta(\mathbf{r})=x^{m} y^{n} z^{k} \exp \left(-\alpha r^{2}\right),
$$

as this choice allows all integrals to be computed analytically.

The difference between the exact energy $E$ and the HF energy is called the correlation energy, $E_{\text {corr }}=E-E_{\mathrm{HF}}$. The correlation energy is often considered as the sum of two contributions, that is, static correlation, which is linked to the insufficiency of a single-reference wave function, and dynamical correlation, which captures repulsion of the electrons beyond the mean-field approximation.

\section{Post Hartree-Fock Methods}

One route to recover the missing correlation energy is to expand the wave function in a linear combination of Slater determinants constructed from a set of orthonormal single-particle orbitals and with an occupation pattern (configuration) different from the Hartree-Fock one, thus obtaining a multireference wave function. In the configuration interaction (CI) approach, the wave function is obtained by considering excitations out of the reference HF determinant to a set of virtual orbitals as

$$
\Psi_{\mathrm{CI}}=c_{0} D_{\mathrm{HF}}+\sum_{a b} c_{a \rightarrow b} D^{a \rightarrow b}+\sum_{a b c d} c_{a b \rightarrow c d} D^{a b \rightarrow c d}+\ldots
$$

where $D^{a \rightarrow b}$ denotes a single excitation where the occupied orbital $a$ in the HF determinant is substituted with the virtual orbital $b$. Similarly, $D^{a b \rightarrow c d}$ indicates a double excitation from $a$ and $b$ to the virtual orbitals $c$ and $d$. Given a basis set for the expansion of the molecular orbitals, the full configuration interaction (full CI) expansion includes every possible configuration obtained by exciting up to $N$ electrons 
in the unoccupied orbitals. In practice, full CI is only feasible for relatively small systems and basis sets since the number of determinants in such an expansion grows exponentially with $N$. One then truncates the expansion to a low level of excitation including the most important configurations: For example, CISD (configuration interaction singles and doubles) only includes single and double excitations and the scaling is reduced to $N^{6}$.

If we denote with $C_{i}$ a spin- and space-adapted configuration state functions (CSF) (i.e. a fixed linear combination of determinants with proper spin and space symmetry), we can rewrite a CI expansion as

$$
\Psi_{\mathrm{CI}}=\sum_{i=1}^{K} c_{i} C_{i}
$$

and, by applying the variational principle, obtain the secular equations for the coefficients $c_{i}$ :

$$
\sum_{j=1}^{K}\left\langle C_{i}|\mathcal{H}| C_{j}\right\rangle c_{j}^{(k)}=E_{\mathrm{CI}}^{(k)} \sum_{j=1}^{K}\left\langle C_{i} \mid C_{j}\right\rangle c_{j}^{(k)},
$$

where $\left\langle C_{i} \mid C_{j}\right\rangle=\delta_{i j}$ as the orbitals are orthonormal.

For a CI expansion (and any linear expansion on a basis set), the solution of these secular equations yields an approximation not only to the ground-state but also to the excited-state wave functions and corresponding energies. The variational principle guarantees that the energy $E_{\mathrm{CI}}^{(0)}$ of the approximate ground-state wave function is an upper bound of the exact ground-state energy, $E_{0}$. Similarly, for excited states which are orthogonal to the ground state and to each other, the HylleraasUndheim-McDonald's theorem states that the approximate solutions with energies $E_{\mathrm{CI}}^{(1)} \leq \ldots \leq E_{\mathrm{CI}}^{(K)}$ satisfy

$$
E_{i} \leq E_{\mathrm{CI}}^{(i)}
$$

where $E_{i}$ are the exact energies of the eigenstates of the Hamiltonian $\mathcal{H}$.

In the multi-configurational self-consistent field (MCSCF) approach, one minimizes the energy not only with respect to the linear coefficients $c_{i}$ but also the LCAO coefficients $a_{j i}$. The complete-active-space self-consistent field (CASSCF) approach is a particular type of MCSCF calculation, where $n$ electrons are distributed over a set of $m$ active orbitals, whose occupancy is allowed to vary. The resulting $\operatorname{CASSCF}(n, m)$ calculation is equivalent to a full CI calculation for $n$ electrons in $m$ orbitals, except that also the orbitals are now optimized to minimize the total energy. In a typical CASSCF calculation, outside the active space, there is a set of inactive orbitals that are always doubly occupied, and a set of virtual orbitals that are always unoccupied. The inactive and virtual orbitals will be important in the multireference perturbation methods described later.

If one is interested in obtaining several states of the same symmetry, it is customary to use a state averaged (SA) MCSCF approach to avoid root-flipping problems in the optimization of the LCAO coefficients. In a SA calculation, the weighted average 
of the energies of the states of interest is optimized:

$$
E_{\mathrm{SA}}=\sum_{I} w_{I} \frac{\left\langle\Psi_{I}|\mathcal{H}| \Psi_{I}\right\rangle}{\left\langle\Psi_{I} \mid \Psi_{I}\right\rangle}
$$

where $\sum_{I} w_{I}=1$ and the states are kept orthogonal through the CI coefficients. The optimization yields a common set of orbitals and the different states differ only in their CI coefficients.

The most important and delicate step in a MCSCF/CASSCF calculation is the selection of the active space, which requires a fair amount of knowledge of the system under study and is rather time-consuming since a great number of trial calculations is often necessary. Furthermore, MCSCF/CASSCF calculations recover only a small part of the correlation energy as relatively small expansions are considered, providing only a limited description of static correlation.

\subsubsection{Perturbation theory}

Rayleigh-Schrödinger perturbation theory (RSPT) can often be successfully applied to improve the results obtained at a certain level of theory, such as Hartree-Fock or MCSCF. The perturbation approach is based on the partition of the total Hamiltonian of the system, $\mathcal{H}$, into a zero-order Hamiltonian, $\mathcal{H}^{(0)}$, and a perturbation operator $\mathcal{V}$, so that

$$
\mathcal{H}=\mathcal{H}^{(0)}+\mathcal{V}
$$

In this framework, the total wave function, $|\Psi\rangle$ and the corresponding energy, $E$, can be written as convergent series expansions:

$$
\begin{aligned}
\left|\Psi_{n}\right\rangle & =\sum_{i=0}^{\infty}\left|\Psi_{n}^{(i)}\right\rangle \\
E_{n} & =\sum_{i=0}^{\infty} E_{n}^{(i)},
\end{aligned}
$$

where the superscript $i$ is the index of the perturbation order and the subscript $n$ refers to an electronic state. To improve the convergence properties of the perturbation series, the zero-order wave function and its corresponding energy should give a good description of the electronic state under analysis. This means that the zero-order Hamiltonian should be as close as possible to the total Hamiltonian thus reducing the role of the perturbation. For any given partition of the Hamiltonian, the following equations hold

$$
\begin{aligned}
E_{n}^{(0)}+E_{n}^{(1)} & =\left\langle\Psi_{n}^{(0)}|\mathcal{H}| \Psi_{n}^{(0)}\right\rangle, \\
E_{n}^{(2)} & =\left\langle\Psi_{n}^{(0)}|\mathcal{H}| \Psi_{n}^{(1)}\right\rangle .
\end{aligned}
$$

Using the expression of the first-order correction to the wave function,

$$
\left|\Psi_{n}^{(1)}\right\rangle=-\sum_{k \neq n}\left|\Psi_{k}^{(0)}\right\rangle \frac{\left\langle\Psi_{k}^{(0)}|\mathcal{H}| \Psi_{n}^{(0)}\right\rangle}{E_{k}^{0}-E_{n}^{0}}
$$


the second-order perturbation correction to the energy becomes

$$
E_{n}^{(2)}=-\sum_{k \neq n} \frac{\left|\left\langle\Psi_{k}^{(0)}|\mathcal{H}| \Psi_{n}^{(0)}\right\rangle\right|^{2}}{E_{k}^{0}-E_{n}^{0}} .
$$

The definition of the perturbation theory is complete once we have decided on the zero-order wave function and Hamiltonian. When the zero-order wave function is chosen to be a Hartree-Fock determinant, the application of RSPT bears the name of Möller-Plesset perturbation theory (MP2). In this case, the zero-order Hamiltonian is defined as the sum of the Fock operators for all the electrons, $\mathcal{H}^{(0)}=\sum_{i} f(i)$, and, assuming a set of canonical orbitals, the zero-order energy is given by the sum of the orbital energies.

If we take a MCSCF wave function to be the zero-order wave function, we obtain the multi-reference perturbation theory (MRPT). In this framework, there is no unique definition of the zero-order Hamiltonian and different choices can be made, obtaining different formulations of the same perturbation theory, which are often characterized by rather different properties. The general idea is to include at the perturbation level the possible excitations not present in the MCSCF wave function, thus generating the eigenstates of the zero-order Hamiltonian (also called perturbation functions) that interact with the reference MCSCF wave function via the total Hamiltonian. The first-order wave function and the second-order correction to the energy are then computed following Equations (2.18)-(2.19).

\section{CASPT2 and NEVPT2}

One of the most popular and employed perturbation approaches, especially in the context of excited-state calculations, is the complete-active-space perturbation theory (CASPT2) $[4,5]$. In this theory, the zero-order wave function is the CASSCF wave function of the state of interest and the perturbation states (i.e. the other zero-order wave functions) are generated by single and double replacements in the CAS wave function. Only these replacement states couple to the zero-order CAS wave function in the calculation of the second-order energy. The resulting excited determinants include, for instance, excitations of one electron from the inactive to the active orbitals, or from the active to the virtuals and all the other possible combinations.

The zero-order Hamiltonian is defined through a generalized one-electron Fock operator projected onto the vector space spanned by the CASSCF wave function and by the perturbation states:

$$
\mathcal{H}^{(0)}=P_{0} \mathcal{F} P_{0}+P_{\mathrm{SD}} \mathcal{F} P_{\mathrm{SD}},
$$

where $P_{0}$ is the projector on the one-dimensional space of the CAS state of interest and $P_{\mathrm{SD}}$ is the projector on the space of the wave functions generated by single and double replacements in the CAS wave function. The Fock operator, $\mathcal{F}$, is defined as

$$
\mathcal{F}=\sum_{p, q}(\mathbf{F})_{p q} a_{p}^{\dagger} a_{q}
$$


where $a_{p}^{\dagger}$ and $a_{q}$ are the creation and the annihilation operator acting on orbital $p$ and $q$, respectively, which can be either inactive, active or virtual. The generalized Fock matrix has elements $\mathbf{F}_{p q}$ given by

$$
(\mathbf{F})_{p q}=h_{p q}+\sum_{r, s}(\mathbf{D})_{r s}\left[\langle p r \mid q s\rangle-\frac{1}{2}\langle p q \mid r s\rangle\right],
$$

where $h_{p q}$ is the core-Hamiltonian matrix and $(\mathbf{D})_{r s}$ is the CASSCF one-particle density matrix. This definition recovers the MP2 approach in the limit of a closed-shell mono-determinantal wave function. The Fock matrix consists of $3 \times 3$ blocks corresponding to the subspaces of the inactive, active, and virtual CASSCF orbitals. The operator $\mathcal{F}$ can be further simplified by separately diagonalizing the three diagonal blocks of the matrix $\mathbf{F}$. This operation is possible thanks to the invariance of the CASSCF space upon unitary transformation of the optimal orbitals within the separate subspaces. The elements of the Fock matrix after this orbital rotation become then $\mathbf{F}_{p q}=\delta_{p q} \epsilon_{p}$ if $p$ and $q$ belong to the same orbital space.

To understand the behavior of this zero-order Hamiltonian, it is convenient to rewrite the diagonal elements of the Fock operator as

$$
(\mathbf{F})_{p p}=-\frac{1}{2}\left[(\mathbf{D})_{p p}(\mathrm{IP})_{p}+\left(2-(\mathbf{D})_{p p}\right)(\mathrm{EA})_{p}\right]
$$

where $(\mathbf{D})_{p p}$ is the occupation of the $p$ orbital and the symbols $(\mathrm{IP})_{p}$ and $(\mathrm{EA})_{p}$ denote the ionization potential (IP) and electron affinity (EA) computed by adding or removing one electron from the $p$ orbital in the CAS state. For doubly occupied and empty orbitals, $(\mathbf{F})_{p p}$ becomes the IP and the EA energy, respectively. For the active orbitals with a variable occupation number, the diagonal terms of the Fock matrix correspond instead to a weighted average of the two quantities. This feature of the Fock operator is undesirable because it overstabilizes the more open-shell configurationis (when active orbitals are involved in the excitation) leading to denominators in Eq. 2.19 that are too small. What one would prefer is that $(\mathbf{F})_{p p}=-(\mathrm{IP})$ for excitations from a partially occupied orbital, and $(\mathbf{F})_{p p}=-(\mathrm{EA})$ for excitation into a partially occupied orbital. To cure this behavior, a shift is introduced in the denominators as $\sigma_{p}^{\mathrm{EA}}=\frac{1}{2}(\mathbf{D})_{p p}\left[(\mathrm{IP})_{p}-(\mathrm{EA})_{p}\right]$ for an excitation into orbital $p$ and $\sigma_{p}^{\mathrm{IP}}=-\frac{1}{2}\left(2-(\mathbf{D})_{p p}\right)\left[(\mathrm{IP})_{p}-(\mathrm{EA})_{p}\right]$ for an excitation out of that orbital. Since the evaluation of $(\mathrm{IP})_{p}-(\mathrm{EA})_{p}$ is not straightforward, a semi-empirical value for this quantity has been determined and set equal to 0.25 a.u. [6], which is often refered to as the IPEA shift in this thesis.

Beside the CASPT2 method, another MRPT has recently been formulated and successfully applied to systems requiring a multireference treatment of the wave function. This method is called the $n$-electron valence-state perturbation theory (NEVPT2) [7, 8] and, differently from CASPT2, makes use of the Dyall's Hamiltonian [9] to define $\mathcal{H}^{(0)}$. The Dyall's Hamiltonian, $\mathcal{H}^{D}$, is equivalent to the Fock operator used in CASPT2 in the core and virtual spaces $\left(\mathcal{H}_{i}^{D}\right)$ but contains bi-electronic contributions within the active space $\left(\mathcal{H}_{a}^{D}\right)$. Assuming the use of canonical orbitals, 
the Dyall's Hamiltonian has the following form:

$$
\begin{aligned}
\mathcal{H}^{D} & =\mathcal{H}_{i}^{D}+\mathcal{H}_{a}^{D} \\
\mathcal{H}_{i}^{D} & =\sum_{i}^{\text {occ }} \epsilon_{i} a_{i}^{\dagger} a_{i}+\sum_{r}^{\text {virt }} \epsilon_{r} a_{r}^{\dagger} a_{r}+C \\
\mathcal{H}_{a}^{D} & =\sum_{a b}^{\text {act }} h_{a b}^{\mathrm{eff}} a_{a}^{\dagger} a_{b}+\frac{1}{2} \sum_{a b c d}^{\text {act }}\langle a b \mid c d\rangle a_{a}^{\dagger} a_{b}^{\dagger} a_{d} a_{c},
\end{aligned}
$$

where $\epsilon_{i}$ and $\epsilon_{r}$ are orbital energies corresponding to the canonical orbitals, $h_{a b}^{\text {eff }}$ represents an averaged interaction between the active electrons and the core ones, and $C$ is an appropriate constant ensuring that $\mathcal{H}^{D}$ is equivalent to the total Hamiltonian within the CAS space [8]. Similarly to Equation 2.20, the zero-order Hamiltonian in NEVPT2 is given by

$$
\mathcal{H}^{(0)}=P_{0} \mathcal{H}^{D} P_{0}+P_{\mathrm{SD}} \mathcal{H}^{D} P_{\mathrm{SD}}
$$

The use of $\mathcal{H}^{D}$ in the definition of the zero-order Hamiltonian guarantees a more balanced treatment of the zero-order reference wave function and of the perturbation functions: Dyall showed that the usage of zero-order energies deriving from a one-electron operator introduces a bias in the energy calculation (resulting in the problems discussed above for the CASPT2 formulation) since the zero-order reference wave function properly takes into consideration the bi-electronic interactions occurring among the active electrons whereas in the perturbation functions these interactions are neglected [9]. The introduction of the bi-electronic interactions in the zero-order Hamiltonian leads, of course, to a higher computational cost, but removes the necessity of introducing a semiempirical correction such as the IPEA shift. Another important consequence is that the occurrence of intruder states is significantly reduced in NEVPT2 with respect to CASPT2. The term "intruder state" is used to identify perturbation states with a zero-order energy close to the reference energy, which creates a singularity in the expansion of the second-order correction to the energy. This is a common problem of the MRPT based on a mono-electronic zeroorder Hamiltonian. In CASPT2, a partial solution is achieved with the introduction of an imaginary level shift in the denominators of the perturbation series, which will remove the intruder states without affecting the zero-order energies of the other perturbation functions $[10,11]$.

\subsubsection{Density Functional Theory}

Density functional theory (DFT) [12] is an alternative to wave-function-based methods, which is very widely employed due to its favorable scaling with system size, allowing the treatment of very large molecular systems. The key quantity in the DFT formalism is the electronic density, $\rho(\mathbf{r})$, a 3-dimensional function, instead of the $3 \mathrm{~N}$ dimensional many-body wave function. The Hohenberg-Kohn theorem [13] lays the 
fundations of DFT and states that the ground-state electron density uniquely determines the external potential and, therefore, the wave function together with all expectation values. Unfortunately, the mapping functional is unknown and must therefore be approximated. In practical DFT applications, one relies on the Kohn-Sham theorem [14], which maps the interacting system of electrons to a non-interacting one with the same ground-state density, and results in the simple single-particle equations:

$$
\left[-\frac{1}{2} \nabla^{2}+v_{\mathrm{eff}}([\rho] ; \mathbf{r})\right] \phi_{i}=\epsilon_{i} \phi_{i}
$$

where the electronic density is constructed by summing over the $N$ occupied orbitals:

$$
\rho(\mathbf{r})=\sum_{i=1}^{N}\left|\phi_{i}(\mathbf{r})\right|^{2} .
$$

The effective Kohn-Sham potential is given by

$$
v_{\text {eff }}([\rho] ; \mathbf{r})=v_{\text {ext }}(\mathbf{r})+\int \frac{\rho\left(\mathbf{r}^{\prime}\right)}{\left|\mathbf{r}-\mathbf{r}^{\prime}\right|} \mathrm{d} \mathbf{r}^{\prime}+v_{\mathrm{xc}}([\rho] ; \mathbf{r}),
$$

where $v_{\text {ext }}(\mathbf{r})$ is the external potential. The exchange-correlation potential $v_{\mathrm{xc}}([\rho] ; \mathbf{r})$ is the functional derivative of the exchange-correlation energy, $E_{\mathrm{xc}}[\rho]$, that enters in the expression of the total energy:

$$
\begin{aligned}
E & =-\frac{1}{2} \sum_{i=1}^{N} \int \phi_{i} \nabla^{2} \phi_{i} \mathrm{~d} \mathbf{r}+\int \rho(\mathbf{r}) v_{\text {ext }}(\mathbf{r}) \mathrm{d} \mathbf{r} \\
& +\frac{1}{2} \iint \frac{\rho(\mathbf{r}) \rho\left(\mathbf{r}^{\prime}\right)}{\left|\mathbf{r}-\mathbf{r}^{\prime}\right|} \mathrm{d} \mathbf{r} \mathrm{d} \mathbf{r}^{\prime}+E_{\mathrm{xc}}[\rho] .
\end{aligned}
$$

Even though Equation 2.29 is in principle exact, the exchange-correlation functional is the aforementioned unknown component of DFT in an actual calculation.

\section{Approximate Exchange-Correlation Functionals}

Many approximate exchange-correlation functionals are available in the literature [15] and recent years have seen a proliferation of new functionals, sometimes even designed for the study of specific properties. In this thesis, we employ the local density approximation (LDA), various generalized gradient approximation (GGA), hybrid, meta-hybrid, and long-range corrected (LC) functionals.

The simplest functional form is given by the local density approximation:

$$
E_{\mathrm{xc}}^{\mathrm{LDA}}[\rho]=\int \rho(\mathbf{r}) \epsilon_{\mathrm{xc}}^{\mathrm{hom}}(\rho(\mathbf{r})) \mathrm{d} \mathbf{r},
$$

where $\epsilon_{\mathrm{xc}}^{\mathrm{hom}}(\rho)$ is the exchange-correlation energy per electron of a uniform electron gas of density $\rho$. Thus, in the LDA, the exchange-correlation energy per electron 
of an inhomogeneous system at a spatial point of density $\rho(\mathbf{r})$ is approximated as the exchange-correlation energy per particle of the uniform electron gas of the same density.

To improve upon LDA, the generalized gradient approximation (GGA) introduces a dependence on the gradient (and possibly higher derivatives) of the density. The general expression of a GGA functional is given by

$$
E_{\mathrm{xc}}^{\mathrm{GGA}}[\rho]=\int \rho(\mathbf{r}) \epsilon_{\mathrm{xc}}^{\mathrm{GGA}}\left(\rho(\mathbf{r}),|\nabla \rho(\mathbf{r})|, \nabla^{2} \rho(\mathbf{r})\right) \mathrm{d} \mathbf{r} .
$$

We will employ the Perdew-Burke-Ernzerhof (PBE) [16] GGA functional in the ground-state $a b$ initio molecular dynamics simulations.

Hybrid functionals introduce an explicit dependence on the Kohn-Sham orbitals by mixing in the functional a portion of exact exchange from Hartree-Fock theory:

$$
E_{\mathrm{x}}^{\mathrm{HF}}[\rho]=-\frac{1}{2} \sum_{i=1}^{N} \sum_{j=1}^{N} \delta_{s_{i}, s_{j}} \iint \frac{\phi_{i}^{*}(\mathbf{r}) \phi_{j}^{*}\left(\mathbf{r}^{\prime}\right) \phi_{j}(\mathbf{r}) \phi_{i}\left(\mathbf{r}^{\prime}\right)}{\left|\mathbf{r}-\mathbf{r}^{\prime}\right|} \mathrm{d} \mathbf{r} \mathrm{d} \mathbf{r}^{\prime} .
$$

where the exchange energy is an implicit functional of the density since the KohnSham orbitals are themselves functionals of the density. The idea of mixing exact exchange in the functional expression was introduced by Becke [17] based on the adiabatic connection formalism (which constructs a path connecting the Kohn-Sham non-interacting system with the interacting one, while keeping the density constant, by linearly switching on the electron-electron interaction). The main benefit of introducing a fraction of exact exchange is to decrease the so-called interaction error of LDA and GGA functionals (for an exact functional, the exchange energy should cancel the Hartree energy in the one-electron limit), which tends to favor too much delocalized densities upon localized ones. One of the most widely employed hybrid functional is B3LYP $[18,19]$, which combines LDA and the Becke-Lee-Yang-Parr (BLYP) [20,21] GGA functional with 20\% exact Hartree-Fock exchange. We use the B3LYP functional for geometry optimization of isolated and solvated chromophores.

Meta-GGA functionals include also a dependence on the local non-interacting kinetic density computed for the Kohn-Sham orbitals:

$$
\tau(\mathbf{r})=\frac{1}{2} \sum_{i}\left|\nabla \phi_{i}(\mathbf{r})\right|^{2} .
$$

Meta-GGA functionals have also been extended to include exact exchange in the so-called meta-hybrid functionals. The four M06 [22-25] functionals are a popular set of meta-GGA's that differ mainly in the amount of exact exchange included: M06-L (0\%), M06 (27\%), M06-2X (54\%), and M06-HF (100\% of exact exchange). From this set, we use the M06-2X functional to optimize ground- and excited-state geometries.

The last family of functionals that we present is the one of the long-range corrected (LC) functionals [26], where the electron-electron interaction is split into a short- and a long-range component as

$$
\frac{1}{r}=\frac{1-g(r)}{r}+\frac{g(r)}{r}
$$


where $g(r)=\operatorname{erf}(\mu r)$ is a standard choice. In the original LC scheme [27,28],

$$
E_{\mathrm{xc}}[\rho]=E_{\mathrm{x}}^{\mathrm{HF}, \mathrm{LR}}[\rho]+E_{\mathrm{x}}^{\mathrm{DFA}, \mathrm{SR}}[\rho]+E_{\mathrm{c}}^{\mathrm{DFA}}[\rho],
$$

where the the exact HF exchange energy is used for the long-range electron-electron interaction $\left(E_{\mathrm{x}}^{\mathrm{HF}, \mathrm{LR}}\right)$, while the short-range interaction $\left(E_{\mathrm{x}}^{\mathrm{DFA}, \mathrm{SR}}\right)$ is treated with a semi-local density functional approximation (DFA) $[15,28]$. The parameter $\mu$ in the error function controls the range of separation and a value in the range of $0.3-0.5$ a.u. is recommended [29]. In this formulation, the LC functionals include $0 \%$ of exact exchange at short range and $100 \%$ at long range. In the more recent formulation of the so-called Coulomb-attenuating method (CAM) [30], a fraction of HF exchange is added at both ranges of separation:

$$
\begin{aligned}
E_{\mathrm{xc}}[\rho] & =a E_{\mathrm{x}}^{\mathrm{HF}, \mathrm{SR}}[\rho]+b E_{\mathrm{x}}^{\mathrm{HF}, \mathrm{LR}}[\rho] \\
& +(1-a) E_{\mathrm{x}}^{\mathrm{DFA}, \mathrm{SR}}[\rho]+(1-b) E_{\mathrm{x}}^{\mathrm{DFA}, \mathrm{LR}}[\rho]+E_{\mathrm{c}}^{\mathrm{DFA}}[\rho],
\end{aligned}
$$

where $E_{\mathrm{x}}^{\mathrm{HF}, \mathrm{SR}}$ is the HF exchange at short range and $E_{\mathrm{x}}^{\mathrm{DFA}, \mathrm{LR}}$ is the DFA exchange energy at long range. The popular CAM-B3LYP functional [30] follows this approach and uses short- and long-range versions of the B88 functional and the same correlation functional used in B3LYP, including 19\% exact exchange at short range ( $a=0.19)$ and $65 \%$ at long range $(b=0.65)$ with $\mu=0.33$ a.u. It has been observed that the use of long-range corrected functionals can improve the well-known shortcoming of other functional forms in describing charge-transfer excitations within time-dependent DFT.

\section{Time-Dependent Density Functional Theory}

A natural question arises whether one can use the energy functional formally developed in the context of a ground-state theory to compute excited states. Perdew and Levy proved that, in the context of pure DFT [31], every extremum of the energy functional corresponds to a stationary-state density. Unfortunately, they also showed that not every stationary-state density is an extremum of the energy functional. Consequently, the formal correctness of the use of a ground-state functional only holds for a (small) subset of excited states. Currently, research effort in the development of excited-state energy functionals is mainly focused on setting up a proper theoretical formulation $[32,33]$ more than generating approximations which can be used in practice.

At present, the most successful theoretical framework for extending DFT to the treatment of excited states is time-dependent density functional theory (TDDFT) [34]. As in the case of DFT, while exact in principle, TDDFT depends on the use of approximate exchange-correlation functionals alongside other approximations mentioned below. In analogy with the Hohenberg-Kohn theorem which is at the roots of DFT, for time-dependent problems, the Runge-Gross theorem [35] proves the biunivocal correspondence between an external time-dependent potential $v_{\text {ext }}(\mathbf{r}, t)$ and the time-dependent electronic density, $\rho(\mathbf{r}, \mathrm{t})$. This leads to the development of a timedependent Kohn-Sham scheme, where the many-body time-dependent Schrödinger 
equation is replaced by a set of equations for non-interacting particles in a timedependent Kohn-Sham effective potential whose orbitals produce the same density of the real system:

$$
\left[-\frac{1}{2} \nabla^{2}+v_{\mathrm{eff}}([\rho] ; \mathbf{r}, t)\right] \phi_{i}(\mathbf{r}, t)=i \frac{\partial}{\partial t} \phi_{i}(\mathbf{r}, t) .
$$

Therefore, the exact electronic density obtained with the Kohn-Sham orbitals is

$$
\rho(\mathbf{r}, t)=\sum_{i=1}^{N}\left|\phi_{i}(\mathbf{r}, t)\right|^{2} .
$$

The Kohn-Sham effective potential is given by:

$$
v_{\text {eff }}([\rho] ; \mathbf{r}, t)=v_{\text {ext }}(\mathbf{r}, t)+\int \frac{\rho\left(\mathbf{r}^{\prime}, t\right)}{\left|\mathbf{r}-\mathbf{r}^{\prime}\right|} \mathrm{d} \mathbf{r}^{\prime}+v_{\mathrm{xc}}([\rho] ; \mathbf{r}, t) .
$$

Importantly, this time-dependent exchange-correlation potential is a different functional of the density than the ground-state one (Eq. 2.28), being the functional derivative of the exchange-correlation component of the action functional $[35,36]$.

Since the time-dependent exchange-correlation potential is unknown, some approximations are required. In the so-called adiabatic approximation, one considers the exchange-correlation potential as local in time, meaning that it reacts instantaneously and without memory to any temporal change of the density:

$$
v_{\mathrm{xc}}^{\mathrm{adiab}}([\rho] ; \mathbf{r}, t)=\left.v_{\mathrm{xc}}^{\mathrm{gs}}([\rho] ; \mathbf{r})\right|_{\rho=\rho(\mathbf{r}, t)},
$$

where $v_{\mathrm{xc}}^{\mathrm{gs}}$ is then chosen as a particular ground-state exchange-correlation potential. Since $v_{\mathrm{xc}}^{\mathrm{gs}}$ is a property of the ground state, this approximation should be more appropriate for time-dependent systems where the density is not too far from the ground-state one.

The most common application of TDDFT is the computation of the response to the perturbation created by an optical field. Knowing the response of the system to this time-dependent perturbation, it is possible to compute within TDDFT the excitation energies of a molecule as the poles of the response function. To achieve this, the key quantity is the linear density-response function, $\chi$, that gives the change in the density of the system subjected to a small perturbation in the external potential:

$$
\delta \rho(\mathbf{r}, \omega)=\int \mathrm{d} \mathbf{r}^{\prime} \chi\left(\mathbf{r}, \mathbf{r}^{\prime}, \omega\right) \delta v_{\text {ext }}\left(\mathbf{r}^{\prime}, \omega\right)
$$

The change in the density can also be expressed using the time-dependent KohnSham scheme as

$$
\delta \rho(\mathbf{r}, \omega)=\int \mathrm{d} \mathbf{r}^{\prime} \chi_{\mathrm{KS}}\left(\mathbf{r}, \mathbf{r}^{\prime}, \omega\right) \delta v_{\mathrm{eff}}\left(\mathbf{r}^{\prime}, \omega\right)
$$

where $\chi_{\mathrm{KS}}$ is the density response function for the non-interacting Kohn-Sham electron system, which we can write in the terms of the unperturbed time-independent 
Kohn-Sham orbitals. To connect the Kohn-Sham to the true response function, we can start from the evaluation of the variation of the Kohn-Sham potential:

$$
\delta v_{\mathrm{eff}}(\mathbf{r}, \omega)=\delta v_{\mathrm{ext}}(\mathbf{r}, \omega)+\int \mathrm{d} \mathbf{r}^{\prime}\left[\frac{1}{\left|\mathbf{r}-\mathbf{r}^{\prime}\right|}+f_{\mathrm{xc}}\left(\mathbf{r}, \mathbf{r}^{\prime}, \omega\right)\right] \delta \rho\left(\mathbf{r}^{\prime}, \omega\right) .
$$

Here, $f_{\mathrm{xc}}\left([\rho] ; \mathbf{r}, \mathbf{r}^{\prime}, \omega\right)$ is the Fourier transform of the exchange-correlation kernel:

$$
f_{\mathrm{xc}}\left([\rho] ; \mathbf{r}, \mathbf{r}^{\prime}, t-t^{\prime}\right)=\frac{\delta v_{\mathrm{xc}}([\rho] ; \mathbf{r}, t)}{\delta \rho\left(\mathbf{r}^{\prime}, t^{\prime}\right)} .
$$

Since, the variation of the Kohn-Sham density $\delta \rho(\mathbf{r}, \omega)$ is equal to the variation of the real system, we can equate Eqs. 2.42 and 2.41, thus obtaining the following Dyson equation of the true response function:

$$
\begin{aligned}
\chi\left(\mathbf{r}, \mathbf{r}^{\prime}, \omega\right) & =\chi_{\mathrm{KS}}\left(\mathbf{r}, \mathbf{r}^{\prime}, \omega\right) \\
& +\int \mathrm{d} \mathbf{x} \int \mathrm{d} \mathbf{x}^{\prime} \chi_{\mathrm{KS}}(\mathbf{r}, \mathbf{x}, \omega)\left[\frac{1}{\left|\mathbf{x}-\mathbf{x}^{\prime}\right|}+f_{\mathrm{xc}}\left(\mathbf{x}, \mathbf{x}^{\prime}, \omega\right)\right] \chi\left(\mathbf{x}^{\prime}, \mathbf{r}^{\prime}, \omega\right) .
\end{aligned}
$$

The response of the interacting system can be obtained via a self-consistent solution if the exact exchange-correlation kernel is known. However, achieving a complete numerical solution of this equation is rather difficult. Instead, one exploits the fact that the excitation energies of the interacting system correspond to the poles of the density-response function, $\chi$. The Kohn-Sham response function $\chi_{\mathrm{KS}}$ has instead poles that coincide with the non-interacting excitation energies, which are given by the difference of Kohn-Sham eigenvalues.

By employing a sequence of algebraic manipulations, linear-response TDDFT is usually expressed in the form of the so-called Casida's equations [37], which is the form used in most quantum chemistry codes. The poles of the response function, $\Omega=E_{m}-E_{0}$, are obtained as solutions of a non-Hermitian eigenvalue problem:

$$
\left[\begin{array}{ll}
\mathbf{A} & \mathbf{B} \\
\mathbf{B} & \mathbf{A}
\end{array}\right]\left(\begin{array}{c}
\vec{X} \\
\vec{Y}
\end{array}\right)=\Omega\left[\begin{array}{cc}
-\mathbf{1} & \mathbf{0} \\
\mathbf{0} & \mathbf{1}
\end{array}\right]\left(\begin{array}{c}
\vec{X} \\
\vec{Y}
\end{array}\right)
$$

where the matrices $\mathbf{A}$ and $\mathbf{B}$ are defined as:

$$
\begin{aligned}
& A_{i a, i^{\prime} a^{\prime}}=\delta_{i i^{\prime}} \delta_{a a^{\prime}}\left(\epsilon_{a}-\epsilon_{i}\right)+K_{i a, i^{\prime} a^{\prime}} \\
& B_{i a, i^{\prime} a^{\prime}}=K_{i a, a^{\prime} i^{\prime}}=\left\langle i a^{\prime} \mid a i^{\prime}\right\rangle+\left\langle i a^{\prime}\left|f_{\mathrm{xc}}\right| a i^{\prime}\right\rangle .
\end{aligned}
$$

The excitation energies are given by the eigenvalues, while the oscillator strengths can be computed from the eigenvectors.

TDDFT is widely employed in the computation of excited-state properties of large molecular systems thanks to its favorable scaling of approximately $\mathcal{O}\left(N^{3}\right)$. Nevertheless, it is important to bear in mind that approximate linear-response TDDFT has various limitations [34]. In particular, it is not suitable to describe excited states with strong multi-configurational character and suffers for the underestimation of charge-transfer excitations [38-40]. This last limitation, however, is largely cured by the use of range-separated functionals. In this work, we will make use of TDDFT either to optimize excited-state geometries or to compute excitation energies in singlepoint calculations, and assess the quality of these results by comparing them with those obtained from wave function-based approaches. 


\subsubsection{Quantum Monte Carlo Methods}

In this thesis, we will extensively use continuous quantum Monte Carlo methods [41], in particular, variational (VMC) and diffusion (DMC) Monte Carlo, to compute excited-state properties of various molecular systems. QMC methods are also wave function based but, differently from traditional quantum chemistry methods, provide a stochastic (approximate) solution of the Schrödinger equation. Thanks to the use of sampling Monte Carlo techniques, QMC benefits from a great freedom in the choice of the functional form of the many-body wave function, which can for instance explicitly depend on the electron-electron distances. The scaling of QMC is also rather favorable with the number of electrons (a mere $N^{4}$ ) and the method is therefore particularly appealing if one aims at treating at the correlated level a fairly large molecular system, which might be too expensive for other approaches such as CASSCF or MRPT.

\section{Variational Monte Carlo}

The variational Monte Carlo method is the simplest flavor of QMC that uses Monte Carlo techniques to evaluate the expectation value of an operator on a given wave function. Let us assume for example that we have a trial wave function $\Psi_{\mathrm{T}}$ and that we are interested in computing the expectation value of the Hamiltonian $\mathcal{H}$ :

$$
E_{V}=\frac{\int \Psi_{\mathrm{T}}^{*}(\mathbf{R}) \mathcal{H} \Psi_{\mathrm{T}}(\mathbf{R}) d \mathbf{R}}{\int \Psi_{\mathrm{T}}^{*}(\mathbf{R}) \Psi_{\mathrm{T}}(\mathbf{R}) \mathrm{d} \mathbf{R}}
$$

where we denote with $\mathbf{R}$ the $3 \mathrm{~N}$ electron coordinates. This expectation value can be rewritten as

$$
E_{V}=\frac{\int\left|\Psi_{\mathrm{T}}(\mathbf{R})\right|^{2}\left[\Psi_{\mathrm{T}}(\mathbf{R})^{-1} \mathcal{H} \Psi_{\mathrm{T}}(\mathbf{R})\right] d \mathbf{R}}{\int\left|\Psi_{\mathrm{T}}(\mathbf{R})\right|^{2} d \mathbf{R}}=\int \rho(\mathbf{R}) E_{L}(\mathbf{R}) \mathrm{d} \mathbf{R},
$$

where

$$
\rho(\mathbf{R})=\frac{\left|\Psi_{\mathrm{T}}(\mathbf{R})\right|^{2}}{\int\left|\Psi_{\mathrm{T}}(\mathbf{R})\right|^{2} \mathrm{~d} \mathbf{R}},
$$

and the local energy is defined as

$$
E_{L}(\mathbf{R})=\frac{\mathcal{H} \Psi_{\mathrm{T}}(\mathbf{R})}{\Psi_{\mathrm{T}}(\mathbf{R})}
$$

In these equations, the function $\rho(\mathbf{R})$ is a positive quantity, integrates to one, and can therefore be interpreted as a probability distribution. Consequently, to compute the integrals, we can resort to classical Monte Carlo techniques and sample a set of configurations $\left\{\mathbf{R}_{m}\right\}$ in the $3 \mathrm{~N}$ dimensional space of electrons, distributed according to $\rho(\mathbf{R})$. The expectation value can then be estimated as an average of the local energy $E_{L}(\mathbf{R})$ evaluated on these configurations:

$$
E_{V} \approx \frac{1}{M} \sum_{m=1}^{M} E_{L}\left(\mathbf{R}_{m}\right)
$$


This derivation is valid for any operator $\mathcal{O}$ diagonal in space representation.

For the realistic molecular systems studied here, the square of the trial wave function is a complicated function in a high-dimensional space and its normalization is not easy to compute. Therefore, we cannot use direct sampling techniques but employ the classical Metropolis algorithm [42] to generate a sequence of configurations $\left\{\mathbf{R}_{m}\right\}$ distributed according to $\rho(\mathbf{R})$. The Metropolis algorithm allows us to sample an arbitrary probability distribution without knowing its normalization. Here, we employ the VMC algorithm as described in Ref. 43, which uses a non-symmetrical transition matrix in the Metropolis algorithm, and which we properly modified to deal with pseudopotentials.

In our calculations, we use trial wave functions of the Jastrow-Slater form, that is, a determinantal expansion multiplied by the so-called correlation Jastrow factor. In a spin-assigned form, the Jastrow-Slater wave function can be written as

$$
\Psi\left(\mathbf{r}_{1}, \ldots, \mathbf{r}_{N}\right)=\mathcal{J}\left(\mathbf{r}_{1}, \ldots, \mathbf{r}_{N}\right) \sum_{k} d_{k} D_{k}^{\uparrow}\left(\mathbf{r}_{1}, \ldots, \mathbf{r}_{N_{\uparrow}}\right) D_{k}^{\downarrow}\left(\mathbf{r}_{N_{\uparrow}+1}, \ldots, \mathbf{r}_{N}\right),
$$

where $D_{k}^{\uparrow}$ and $D_{k}^{\downarrow}$ are Slater determinants of single particle orbitals for the upand down-spin electrons, respectively, and the Jastrow factor $\mathcal{J}$ describes electronelectron as well as higher-body correlations (e.g. through the inclusion of electronelectron-nucleus terms). The presence of the Jastrow factor also ensures that the Kato's cusp conditions are satisfied at the inter-particle coalescence points. When the interparticle distance goes to zero, the electron-electron and electron-nucleus potentials diverge as $1 / r$ and $-Z / r$, respectively. Since the local energy must be finite, the kinetic energy must have an opposite divergence to the potential. This cancellation can be enforced if the trial wave function satisfies the so-called "cusp conditions" and displays an appropriate discontinuity at the coalescence points. The cusp condition for the coalescence of two electrons of opposite spin is

$$
\left.\frac{1}{\Psi} \frac{\partial \hat{\Psi}}{\partial r_{i j}}\right|_{r_{i j}=0}=\frac{1}{2}
$$

and the condition for the coalescence of an electron with a nucleus of charge $Z$ placed at the origin is

$$
\left.\frac{1}{\Psi} \frac{\partial \hat{\Psi}}{\partial r_{i}}\right|_{r_{i}=0}=-Z
$$

where $\hat{\Psi}$ is the spherical average of the wave function over an infinitesimally small sphere centered at zero. Note that the condition is obtained if the wave function is different than zero at the coalescence point. The Kato's cusp conditions can be further generalized to the case when the wave function goes to zero (as for two spinlike electrons). Methods such as configuration interaction are characterized by a slow convergence in the expansion over Slater determinants in part because of the difficulty in describing the cusp at the electron-electron coalescence points (i.e. they give a poor description of so-called dynamical correlation). 
With the Jastrow factor, the explicit dependence on the inter-electronic distances is introduced in the wave function and the cusp conditions can be exactly imposed, recovering an important component of dynamical correlation. Therefore, the use of quantum Monte Carlo techniques to compute expectation values allows us to employ a much more compact representation of the wave function such as the JastrowSlater one, which is usually expanded over few hundred determinants, while in a CI calculation one would need an expansion over millions of determinants. For most applications, we use the following simple form for the Jastrow factor:

$$
\mathcal{J}\left(\mathbf{r}_{1}, \ldots, \mathbf{r}_{N}\right)=\prod_{\alpha, i} \exp \left\{A\left(r_{i \alpha}\right)\right\} \times \prod_{i<j} \exp \left\{B\left(r_{i j}\right)\right\}
$$

in which $A\left(r_{i \alpha}\right)$ depends on the electron-nucleus distances, $r_{i \alpha}$, and $B\left(r_{i j}\right)$ on the electron-electron distances, $r_{i j}$. Both functions are fifth-order polynomials and the arguments are scaled as $\bar{r}=\left(1-e^{\kappa r}\right) / \kappa$ to ensure that the Jastrow factor behaves well at large interparticle distances. The function $B\left(r_{i j}\right)$ includes an additional $b_{1} \bar{r}_{i j} /\left(1+b_{2} \bar{r}_{i j}\right)$ term to satisfy the cusp conditions with $b_{1}=1 / 2$ and $1 / 4$ for opposite- and like-spin electrons, respectively. Different polynomial parameters are used for different atom types in the case of the electron-nucleus terms and, since we use pseudopotentials, we do not need to impose the electron-nucleus cusp conditions. We also include additional electron-electron-nucleus "three-body" terms in the Jastrow factor to perform geometry optimizations and to test the effect of the use of higher-body correlation on the quality of the optimized structures.

The quality of the trial wave function does not only directly affect the quality of the expectation values computed with it, but also the statistical efficiency of the VMC (and DMC) algorithm as well as the final accuracy of the results obtained in DMC as explained below. It is therefore important that the wave function captures the "right" physics of the system, for instance, in the choice of the excitations included in the determinantal expansion to describe static correlation. Furthermore, the parameters in the Jastrow factor should be optimized together with the parameters in the determinantal component (CI and LCAO coefficients) since the optimal values will be different from the initial DFT or MCSCF ones, which have been obtained in the absence of the Jastrow factor to construct an initial wave function. For the optimization of the parameters in the trial wave function of a system in its ground state, we use the so-called linear optimization method within energy minimization [44] (the method is similar to the super-CI optimization method used in MCSCF, which has been extended to arbitrary wave functions in the framework of variational Monte Carlo).

Since we are also interested in non-trivial excited states of the same symmetry as other lower-energy states, we face here the same problems one encounters in MCSCF when optimizing multiple states of the same symmetry. To obtain a stable optimization procedure, we therefore employ a generalization of the linear method to state-average (SA) optimization [45] and, in the spirit of SA-MCSCF, optimize the parameters of the multiple (ground and excited) orthogonal states described by the 
wave functions:

$$
\Psi_{I}=\sum_{i=1}^{N_{\mathrm{CSF}}} c_{i}^{I} \mathcal{J} C_{i},
$$

which share the same Jastrow factor and orbitals but have different linear coefficients, by minimizing the state-average energy

$$
E_{\mathrm{SA}}=\sum_{I} w_{I} \frac{\left\langle\Psi_{I}|\mathcal{H}| \Psi_{I}\right\rangle}{\left\langle\Psi_{I} \mid \Psi_{I}\right\rangle},
$$

with $\sum_{I} w_{i}=1$. The optimization produces a set of orbitals and a Jastrow factor which give a comparably good description of the states under considerations, while preserving orthogonality among the states through the CI coefficients.

\section{Diffusion Monte Carlo}

VMC is a very powerful approach which allows us to use any wave function as long as is computable in a reasonable amount of time. VMC can be very effectively used to understand the system of interest by exploring which type of correlation is important and to construct a high-quality wave function via optimization of its parameters. With QMC, it is also possible to improve on this wave function with the use of a projector Monte Carlo technique, which removes (at least in part) the bias introduced by the trial wave function from the results. The idea of projection Monte Carlo is to construct an operator that inverts the spectrum of the Hamiltonian $\mathcal{H}$ and to project out all excited states by the repeated application of this operator to an initial trial wave function. The final result of this procedure will be the ground state of the given Hamiltonian. Diffusion Monte Carlo (DMC) is a particular projection approach, which we use in this thesis.

In DMC, the projection operator is chosen to be $\exp \left[-\tau\left(\mathcal{H}-E_{\mathrm{T}}\right)\right]$, where $E_{\mathrm{T}}$ is a constant, whose role will soon become clear, and $\tau$ the time-step. If we subsequently apply this operator to an initial trial wave function, we obtain the sequence of wave functions:

$$
\Psi^{(n)}=e^{-\tau\left(\mathcal{H}-E_{\mathrm{T}}\right)} \Psi^{(n-1)} .
$$

If we expand the initial wave function $\Psi^{(0)}$ on the eigenstates $\Psi_{i}$ with energies $E_{i}$ of $\mathcal{H}$, we obtain for $\Psi^{(n)}$ :

$$
\Psi^{(n)}=\sum_{i} \Psi_{i}\left\langle\Psi^{(0)} \mid \Psi_{i}\right\rangle e^{-n \tau\left(E_{i}-E_{\mathrm{T}}\right)},
$$

Since the coefficients of the excited states die off exponentially fast relative to the one of the ground state, we obtain:

$$
\lim _{n \rightarrow \infty} \Psi^{(n)}=\Psi_{0}\left\langle\Psi^{(0)} \mid \Psi_{0}\right\rangle e^{-n \tau\left(E_{0}-E_{\mathrm{T}}\right)} .
$$


Therefore, if we adjust the trial energy $E_{\mathrm{T}} \approx E_{0}$ to keep the overall normalization of $\Psi^{(n)}$ fixed, the projection gives us the ground state $\Psi_{0}$ of the Hamiltonian.

To understand how to perform the projection in practice, let us rewrite Eq. 2.58 in integral form as:

$$
\Psi^{(n)}\left(\mathbf{R}^{\prime}, t+\tau\right)=\int \mathrm{d} \mathbf{R} G\left(\mathbf{R}^{\prime}, \mathbf{R}, \tau\right) \Psi^{(n-1)}(\mathbf{R}, t),
$$

where the Green's function is defined as:

$$
G\left(\mathbf{R}^{\prime}, \mathbf{R}, \tau\right)=\left\langle\mathbf{R}^{\prime}\left|e^{-\tau\left(\mathcal{H}-E_{\mathrm{T}}\right)}\right| \mathbf{R}\right\rangle .
$$

If we can sample the trial wave function and the Green's function in Eq. 2.61, we can compute this integral stochastically by Monte Carlo integration. Unfortunately, since electrons are fermions, we cannot interpret the antisymmetric wave function as a probability distribution. Nevertheless, for the moment, let us assume that we are dealing with a bosonic system and return to this problem later.

Let us now check that we can treat the Green's function as a transition probability. For small $\tau$, the Green's function can be approximated via the Trotter-Suzuki formula applied to the operator $\mathcal{H}=\mathcal{T}+\mathcal{V}$ as

$$
\left\langle\mathbf{R}^{\prime}\left|e^{-\mathcal{H} \tau}\right| \mathbf{R}\right\rangle \approx \frac{1}{(2 \pi \tau)^{3 N / 2}} \exp \left[-\frac{\left(\mathbf{R}^{\prime}-\mathbf{R}\right)^{2}}{2 \tau}\right] \exp [-\tau \mathcal{V}(\mathbf{R})]
$$

The first factor is a Gaussian, which is positive, can be interpreted as a distribution probability, and easily sampled. The exponential of the potential $\mathcal{V}(\mathbf{R})$ is again positive but does not preserve normalization and is a factor by which we multiply the wave function $\Psi(\mathbf{R}, t)$ at each step. Combining both factors, we can interpret the iteration in Eq. 2.61 as a branching random walk, where the walkers perform a diffusion step and their weights are then rescaled by the positive exponential factor (the weight grows in regions where the potential is less than the average energy, and is reduced in regions of high potential). Since the short-time expression of the Green's function is valid only for $\tau \rightarrow 0$, the results of a DMC calculation must be extrapolated in the limit of small $\tau$.

The simple algorithm described above is in fact very inefficient: The potential can vary significantly in configuration space or even diverge as in the case of the Coulomb potential. It is however possible to overcome these difficulties by using the so-called importance sampling algorithm [46] and making use of the good trial wave function $\Psi_{\mathrm{T}}$ we have constructed in VMC. To do so, we start from Eq. 2.61, multiply each side by $\Psi_{\mathrm{T}}$, and work with the probability distribution $f^{(n)}(\mathbf{R})=\Psi_{\mathrm{T}}(\mathbf{R}) \Psi^{(n)}(\mathbf{R})$ which satisfies

$$
f^{(n)}\left(\mathbf{R}^{\prime}, t+\tau\right)=\int \mathrm{d} \mathbf{R} \tilde{G}\left(\mathbf{R}^{\prime}, \mathbf{R}, \tau\right) f^{(n-1)}(\mathbf{R}, t) .
$$

The importance-sampled Green's function is given by:

$$
\tilde{G}\left(\mathbf{R}^{\prime}, \mathbf{R}, \tau\right)=\Psi_{\mathrm{T}}\left(\mathbf{R}^{\prime}\right)\left\langle\mathbf{R}^{\prime}\left|e^{-\tau\left(\mathcal{H}-E_{\mathrm{T}}\right)}\right| \mathbf{R}\right\rangle / \Psi_{\mathrm{T}}(\mathbf{R}),
$$


and, for small $\tau$, becomes the drift-diffusion-branching short-time Green's function:

$$
\begin{array}{r}
\tilde{G}\left(\mathbf{R}^{\prime}, \mathbf{R}, \tau\right)=(2 \pi \tau)^{3 N / 2} \exp \left[-\frac{\left(\mathbf{R}^{\prime}-\mathbf{R}-\tau \mathbf{V}(\mathbf{R})\right)^{2}}{2 \tau}\right] \times \\
\times \exp \left\{-\tau\left[\left(E_{\mathrm{L}}(\mathbf{R})+E_{\mathrm{L}}\left(\mathbf{R}^{\prime}\right)\right) / 2-E_{\mathrm{T}}\right]\right\}+O\left(\tau^{2}\right)
\end{array}
$$

where the quantum velocity is defined as

$$
\mathbf{V}(\mathbf{R})=\frac{\nabla \Psi_{\mathrm{T}}(\mathbf{R})}{\Psi_{\mathrm{T}}(\mathbf{R})}
$$

and $E_{\mathrm{L}}(\mathbf{R})$ is the local energy for the trial wave function $\Psi_{\mathrm{T}}$. There are two new important features in this Green's function: The quantum velocity pushes the walkers towards regions where the wave function is large and the local energy $E_{\mathrm{L}}(\mathbf{R})$ appears in the branching factor instead of the bare potential $\mathcal{V}(\mathbf{R})$. If the trial wave function approaches an eigenstate of the Hamiltonian, the local energy becomes constant and equal to the eigenvalue. Consequently, a good trial wave function will result in smaller fluctuations in the branching factor. For example, the instabilities due to the divergences of the Coulomb potential at the interparticle coalescence points are removed since the wave function is constructed to obey the cusp conditions.

We still need to address one important point: Previously, we have assumed that the system is bosonic, while the electrons are fermions and their antisymmetric wave function changes sign and cannot be treated as a probability distribution. Algorithms which attempt to handle both signs of the wave function lead to the so-called fermion sign problem: The bosonic component (lower in energy) grows at the expense of the fermionic one, and the antisymmetric signal is lost in the noise. To avoid this problem, we work in the so-called fixed-node approximation and impose in the evolution the boundary condition that the solution $\Psi(\mathbf{R}, t)$ has the same nodes (the same zeros) as the given trial wave function (for instance, by forbidding moves in which the trial wave function changes sign). The Schrödinger equation is then solved exactly inside the nodal regions but not at the nodes where the derivatives of the solution will be discontinuous. The fixed-node solution is exact only if the nodes of the trial wave function are exact and, for approximate nodes, gives an upper bound to the exact energy.

Importantly for this thesis, DMC can also be used to study the excited states of a given Hamiltonian. If the state is the lowest state of a one-dimensional representation of the point group of the molecule under study, this is equivalent to a ground-state problem and DMC will yield a solution which is variational. If the excited state is not the lowest state in its symmetry, we no longer have a variational principle but can still use DMC in the fixed-node approximation and employ the nodes of a trial excitedstate wave function to keep the solution from collapsing on lower-energy states. We are however only guaranteed to obtain the exact excited-state solution if the nodes of the trial wave function are exact. In practice, we find that, unless one uses an intentionally poor excited-state wave function, DMC yields excited-state energies which approach the reference value from above as the quality of the excited-state trial wave function is improved. 


\subsection{Multiscale Techniques}

In this thesis, we will also investigate the excited-state properties of molecules in solution or embedded in a protein. For these systems, a full quantum mechanical treatment is impossible and one must rely on a simplified treatment where the main component responsible for light absorption (the chromophore) is described quantum mechanically, while the rest of the protein is described at a lower level of theory. In paritioning the system, we are assuming that the excitation is spatially localized, an assumption which is not always valid (as discussed later) but which results in a significant reduction of the computational cost. The larger environment surrounding the photoexcited region will here be simulated with less expensive classical methods.

Within quantum-in-classical methods, different formulations with various levels of sophistication can be employed to describe the environment, either preserving its explicit atomistic structure [47-50] or, at the other extreme, as a continuum medium [50-53]. If the environment is homogeneus, like in the case of a solution, and one aims to study the average bulk effects on a photoactive solute, a cost-effective approach is represented by the polarizable continuum model (PCM) [51,52]. In this model, the solute is enclosed in a cavity and the solvent outside the cavity is described as a continuum dielectric medium. In practice, the solute interacts with point charges distributed on the surface (and sometimes also in the bulk) of the continuum medium $[51,54]$ and induced by the electronic density of the solute. The PCM model will be employed in the study of the effect of water solvent on the excited-state optimized geometries of small prototypical chromophores.

If the system presents instead specific local interactions, like hydrogen bonds, which affect the excited state of the embedded region, it is necessary to provide a description of the environment, which preserves its discrete nature $[49,55]$. In this case, the simplest classical description of the atoms of the environment is through the use of partial atomic charges, which perturb the photoactive subsystem with a static external electric field. This so-called quantum mechanics-in-molecular mechanics (QM/MM) hybrid approach has been often used in the study of excited states, but recent work by several groups on small organic molecules in solution as well as photoactive proteins [56-70] has provided substantial evidence that static point-charge embedding, although qualitatively able to reproduce some experimental findings, appears inadequate if one strives for higher accuracy.

To achieve a more realistic and accurate modeling of the chromophore-environment interactions, it is possible to formulate a more sophisticated classical embedding scheme which takes into account polarization effects between the two subsystems, both in the ground state and in response to the excitation of the embedded quantum region. This can be done by describing the classical atoms not only with partial atomic charges but also with induced dipoles determined by the atomic polarizabilities and the total electric field of the system with contributions from the embedded electronic density, the static electric field of the point charges, and the other induced dipoles in the environment. The quantum part is therefore polarized by the classical region, which can now respond to the presence of the embedded molecule and to the variations of its electronic density upon excitation [71-74]. We will call 
this polarizable embedding scheme QM/MMpol to underline the difference with the traditional static embedding. In this thesis, we will explore the use of QM/MMpol approach both in its state-specific and linear-reponse formulation for the description of the vertical excitation energies of small chromophores in water solution and of the protein rhodopsin as well as develop and implement a QMC/MMpol model.

\subsubsection{The polarizable continuum model}

In the PCM, the chromophore is placed inside a cavity made of a set of interlocking spheres generally centered on the atoms, which forms a boundary between the quantum and the classical part. The environment (in our application a homogeneus solvent) is represented as a continuum medium characterized by a static dielectric constant, $\epsilon$, while the dielectric constant inside the cavity is equal to one. The Poisson equation couples the electric field and the charge density of the solute with the electric field and the charge density of the solvent:

$$
\nabla \cdot \mathbf{E}=4 \pi \rho^{\mathrm{tot}}(\mathbf{r})
$$

where $\mathbf{E}$ is the total electric field and $\rho^{\text {tot }}(\mathbf{r})$ is the total charge density of the electrons and nuclei of the solute and the polarization density of the solvent. The electric field of the continuum medium is generated by a charge distribution that can be either placed on the surface of the cavity only, or also in the bulk of the continuum medium (volume charges), to accurately describe the solvent polarization effects arising from the spill-out of the solute electronic density through the tails of the wave function.

The surface polarization charge density, $\sigma$, is linked to the total electric field as

$$
\sigma(\mathbf{r})=\frac{1-\epsilon}{4 \pi \epsilon} \mathbf{n}_{+} \cdot \mathbf{E}_{-}(\mathbf{r})
$$

where $\mathbf{n}_{+}$is a versor pointing outside the cavity and $\mathbf{E}_{-}(\mathbf{r})$ is the total electric field evaluated at the surface immediately inside the cavity, with $\mathbf{E}(\mathbf{r})=\mathbf{E}_{\text {solute }}(\mathbf{r})+$ $\mathbf{E}_{\mathrm{surf}}(\mathbf{r})+\mathbf{E}_{\mathrm{vol}}(\mathbf{r})$. The surface charge distribution is discretized dividing the surface in elements (tesserae) of area $a$ and placing point charges at the center of each of them. This allows us to approximate the surface charge density at the point $\mathbf{r}_{\mathbf{k}}$ as

$$
\sigma\left(\mathbf{r}_{k}\right) \approx \frac{q_{k}}{a}
$$

where the positions of the point charges are determined by minimizing the mutual repulsion of equal charges on the surface of a sphere of a given fixed radius [75]. When included in the model, the volume polarization charge density outside the cavity is given by

$$
\rho_{\mathrm{vol}}^{(\mathrm{pol})}(\mathbf{r})=\left(\frac{1}{\epsilon}-1\right) \rho_{\mathrm{e}}(\mathbf{r})
$$

where $\rho_{\mathrm{e}}(\mathbf{r})$ is the electronic charge density of the solute. In practical implementations, the volume charge density is also represented as a set of discrete point charges. 
The surface polarization charges, $q_{k}$, are induced by the electric field of the solute and the volume charge distribution, and obey the relationship derived from the Poisson equation:

$$
q_{k}=\sum_{j} G_{k j}(\Sigma, \epsilon) \mathbf{n}_{+} \cdot\left[\mathbf{E}_{\mathrm{vol}}\left(\mathbf{r}_{j}\right)+\mathbf{E}_{\mathrm{solute}}\left(\mathbf{r}_{j}\right)\right]
$$

where $G_{k j}$ depends only on the shape of the cavity surface, $\Sigma$, and on the solvent dielectric constant.

This equation must be brought to self-consistency with the molecular wave function of the solute, which is in turn optimized in the presence of the PCM environment. This is achieved with the minimization of the energy functional:

$$
\begin{aligned}
E[\Psi] & =\left\langle\Psi\left|\mathcal{H}_{\mathrm{QM}}\right| \Psi\right\rangle+\sum_{\alpha<\beta} \frac{Z_{\alpha} Z_{\beta}}{\left|\mathbf{R}_{\alpha}-\mathbf{R}_{\beta}\right|} \\
& +\frac{1}{2} \int \rho_{\text {solute }}(\mathbf{r})\left[V_{\sigma}(\mathbf{r})+V_{\text {vol }}(\mathbf{r})\right] \mathrm{d} \mathbf{r}
\end{aligned}
$$

where $\mathcal{H}_{\mathrm{QM}}$ is the electronic Hamiltonian of the isolated solute and $\rho_{\text {solute }}$ includes contributions from the nuclei and the electrons.

\subsubsection{QM/MM and QM/MMpol models}

If the system requires a more realistic description of the environment and expanding the quantum region is not a computationally viable option, it is often possible to obtain quantitative results for the excited-state properties of the embedded region using a discrete classical embedding approach. In the simplest quantum mechanicalin-molecular mechanics (QM/MM) model, the atoms of the environment are replaced by classical point charges creating a static external electric field which perturbs the quantum region. The Schrödinger equation for the solute wave function becomes

$$
\left(\mathcal{H}_{\mathrm{QM}}+\mathcal{V}\right) \Psi=\mathrm{E} \Psi
$$

with $\mathcal{H}_{\mathrm{QM}}$ the Hamiltonian of the isolated quantum part and $\mathcal{V}$ the potential describing the interaction with the classical region:

$$
\mathcal{V}=\sum_{\mathrm{m}}^{\mathrm{N}_{\mathrm{ch}}} q_{\mathrm{m}} \hat{V}_{\text {chro }}\left(\mathbf{r}_{\mathrm{m}}\right)
$$

where $\hat{V}_{\text {chro }}\left(\mathbf{r}_{\mathrm{m}}\right)$ is the electrostatic potential operator generated by the electrons and the nuclei of the quantum part at the coordinate $\mathbf{r}_{\mathrm{m}}$ of the point charge $q_{\mathrm{m}}$. The energy of the combined system for an electronic state described by a wave function $\Psi$ is given by

$$
\mathrm{E}[\Psi]=\left\langle\Psi\left|\mathcal{H}_{\mathrm{vac}}+\mathcal{V}\right| \Psi\right\rangle+\mathrm{U}_{\mathrm{env}}
$$


where the term $U_{\text {env }}$ represents a constant shift in the total energy, which does not influence the excitation energy and is given by

$$
\mathrm{U}_{\mathrm{env}}=\frac{1}{2} \sum_{\mathrm{n}}^{\mathrm{N}_{\mathrm{ch}}} \sum_{\mathrm{m} \neq \mathrm{n}}^{\mathrm{N}_{\mathrm{ch}}} \frac{q_{\mathrm{m}} q_{\mathrm{n}}}{\left|\mathbf{r}_{\mathrm{m}}-\mathbf{r}_{\mathrm{n}}\right|}
$$

A clear limit of such a static embedding model is that the classical part produces a perturbation on the quantum system but cannot respond to a variation of its electronic distribution. The ability of the environment to relax in response to the excitation of the chromophore turns out to be important to accurately reproduce the excitation energies of molecules which strongly couple to the environment, for instance, for an excitation characterized by charge-transfer character and a large variation of the molecular dipole moment as we will show in Chapters 5 and 6.

More flexible embedding conditions can be obtained by including polarizable dipoles along with the static charges in a so-called QM/MMpol scheme. The use of polarizable dipoles is not computationally much more expensive and allows one to polarize the environment to the ground state of the active site for an enhanced description of its surroundings and also to include the response of the environment to the excitation of the embedded region by polarizing the dipoles with respect to the excited chromophore. The induced dipole at site $a$ in equilibrium with the quantum system depends linearly on the total electric field, E, through the isotropic polarizability, $\alpha_{a}$, as

$$
\begin{aligned}
\boldsymbol{\mu}_{\mathrm{a}} & =\alpha_{\mathrm{a}} \mathbf{E}\left(\mathbf{r}_{\mathrm{a}}\right) \\
& =\alpha_{\mathrm{a}}\left[\left\langle\Psi\left|\hat{\mathbf{E}}_{\text {chro }}\left(\mathbf{r}_{\mathrm{a}}\right)\right| \Psi\right\rangle+\sum_{\mathrm{b} \neq \mathrm{a}} \frac{q_{\mathrm{b}}\left(\mathbf{r}_{\mathrm{a}}-\mathbf{r}_{\mathrm{m}}\right)}{\left|\mathbf{r}_{\mathrm{a}}-\mathbf{r}_{\mathrm{m}}\right|^{2}}-\mathbf{T}_{\mathrm{ab}} \cdot \boldsymbol{\mu}_{\mathrm{b}}\right],
\end{aligned}
$$

where the total electric field at site $a$ is generated by the quantum (nuclear and electronic) system, $\hat{\mathbf{E}}_{\text {chro }}\left(\mathbf{r}_{\mathrm{a}}\right)$, the static charges, and the other dipoles. The interaction tensor $\mathbf{T}$ is equal to:

$$
\left(\mathbf{T}_{\mathrm{ab}}\right)_{i j}=\frac{1}{r_{\mathrm{ab}}^{3}}\left[\delta_{i j}-3 \frac{\left(\mathbf{r}_{a b}\right)_{i}\left(\mathbf{r}_{\mathrm{ab}}\right)_{j}}{r_{\mathrm{ab}}^{2}}\right] .
$$

In Eqs. 2.74- 2.76, the term describing the interaction between the quantum and the classical part becomes

$$
\mathcal{V}=\sum_{\mathrm{m}}^{\mathrm{N}_{\mathrm{ch}}} q_{\mathrm{m}} \hat{V}_{\text {chro }}\left(\mathbf{r}_{\mathrm{m}}\right)-\sum_{\mathrm{a}}^{\mathrm{N}_{\mathrm{dip}}} \boldsymbol{\mu}_{\mathrm{a}} \cdot \hat{\mathbf{E}}_{\mathrm{chro}}\left(\mathbf{r}_{\mathrm{a}}\right),
$$

and the contribution of the environment to the energy is now given by

$$
\begin{aligned}
\mathrm{U}_{\text {env }} & =\frac{1}{2} \sum_{\mathrm{n}}^{\mathrm{N}_{\mathrm{ch}}} \sum_{\mathrm{m} \neq \mathrm{n}}^{\mathrm{N}_{\mathrm{ch}}} \frac{q_{\mathrm{m}} q_{\mathrm{n}}}{\left|\mathbf{r}_{\mathrm{m}}-\mathbf{r}_{\mathrm{n}}\right|}-\sum_{a}^{\mathrm{N}_{\text {dip }}} \boldsymbol{\mu}_{\mathrm{a}} \cdot \mathbf{E}_{q}\left(\mathbf{r}_{\mathrm{a}}\right) \\
& +\frac{1}{2} \sum_{\mathrm{a}}^{\mathrm{N}_{\text {dip }}} \sum_{\mathrm{b} \neq \mathrm{a}}^{\mathrm{N}_{\text {dip }}} \boldsymbol{\mu}_{\mathrm{a}} \cdot \mathbf{T}_{\mathrm{ab}} \cdot \boldsymbol{\mu}_{\mathrm{b}}+\frac{1}{2} \sum_{\mathrm{a}}^{\mathrm{N}_{\text {dip }}} \frac{\mu_{\mathrm{a}}^{2}}{\alpha_{\mathrm{a}}},
\end{aligned}
$$


where the first term, as in the case of the static embedding, describes the interactions of the point charges, the second term the interaction of the induced dipoles with the electric field of the point charges, the third the dipole-dipole interaction, and the last one the self-energy of the dipoles, which is the work exerted by the electric field to induce the dipoles. In these conditions, the dipoles $\boldsymbol{\mu}$ and the wave function $\Psi$ are mutually dependent and must be brought to self-consistency. This procedure results in a different sets of induced dipoles for the ground and the excited states. This means that one has to solve a non-linear the Schrödinger equation (Eq. 2.74), coupled with the classical equation for the dipoles (Eq. 2.78), where the potential $\mathcal{V}$ depends on the wave function quadratically through the induced dipoles. Solving this equation leads to a set of non-orthogonal wave functions in equilibrium with their corresponding dipoles.

It is possible to follow several routes to compute the excited states of a quantum system embedded in polarizable dipoles. One possibility is to polarize the dipoles to the ground state of the quantum system (polGS) and then freeze the dipole values in the calculation of the excited states. This is still a form of static embedding, but the ground-state description is obviously improved with respect to the use of point charges only. The second choice is to allow the environment to respond to the electronic excitation of the embedded species. This can be achieved polarizing the quantum system using different set of dipoles, brought to self consistency with the wave function of the ground and the excited state independently (polSS). This last polarization regime fully recovers the differential electrostatic effects on the two states involved in the electronic excitation and leads to an intuitive interpretation of the observed results: The bigger the difference between the ground- and the excitedstate electronic distribution, the bigger is the correction on the excitation energy of polSS embedding with respect to polGS.

Another possibility to account for relaxation effects is based on linear-response theory (polLR) and determines the excitation energies as poles in the dynamic polarizability of the system. While state-specific and linear-response-based approaches give identical excitations for an isolated quantum calculation (with a potential which is linear in the wave function), the two ways of computing excited states (polSS and polLR) in the quadratic potential $\mathcal{V}(\Psi)$ yield different excitation energies, even in the limit of the exact solution of the Schrödinger equation. An analysis using firstorder perturbation theory reveals that the polSS and polLR excitation energies behave as [76]:

$$
\frac{\Delta E_{\mathrm{polSS}}-\Delta E_{\mathrm{polGS}}}{\Delta E_{\mathrm{polLR}}-\Delta E_{\mathrm{polGS}}} \approx \frac{\left|\boldsymbol{\mu}_{1}-\boldsymbol{\mu}_{0}\right|^{2}}{2 \mu_{01}^{2}}
$$

where $\boldsymbol{\mu}_{I}=\left\langle\Psi_{I}\left|\sum_{i} \hat{\mathbf{r}}_{i}\right| \Psi_{I}\right\rangle$ is the expectation value of the electronic dipole moment for state $I$ and $\mu_{01}=\left\langle\Psi_{0}\left|\sum_{i} \hat{\mathbf{r}}_{i}\right| \Psi_{1}\right\rangle$ the transition dipole moment (TDM) of the excitation. For a given system and excitation, the change in dipole moment and the TDM can have sizable differences and, consequently, also the change in excitation with respect to the polGS value can significantly differ in the two schemes. In the polLR approach, the dipoles in the environment are polarized to the transition density of the system and the polLR contribution corresponds to a dispersion-like coupling 
between the quantum oscillations of the excitation and the classical dipoles in the environment [77].

\subsection{Computational Details}

We list here the codes employed in the various calculations. Computational details specific to the calculations will be given in each chapter.

The Molcas [78] code is a quantum chemistry code, based on Gaussian basis sets, that we use to perform CASSCF and CASPT2 calculations.

The Gaussian 09 [79] code is a quantum chemistry code we use to perform ground-state DFT and linear-response TDDFT calculations as well as well optimize geometries in the ground and excited states. It employes Gaussian basis sets and a wide range of exchange-correlation functionals is available.

The GAMESS [80] code is also an ab initio quantum chemistry package which we mostly use to generate the CASSCF component of the starting QMC wave functions either in state-specific or SA-CASSCF calculation. This code employs Gaussian basis sets.

Other packahes used in this thesis are the CFOUR [81] code for coupled-cluster calculations, and the ORCA [82] code for NEVPT2 calculations. Both codes employ Gaussian basis sets.

Finally, the code CHAMP is used for all the quantum Monte Carlo calculations. It can perform VMC and DMC calculations, and optimizes the wave function parameters by energy minimization. 


\section{Bibliography}

[1] C. J. Cramer, Essentials of Computational Chemistry, 2nd ed. (Wiley, 2004).

[2] F. Jensen, Introduction to Computational Chemistry, 2nd ed. (Wiley, 2007).

[3] A. Szabo and N. S. Ostlund, Modern quantum chemistry: Introduction to advanced electronic structure theory (Dover, 1996), pp. 127-128.

[4] K. Andersson, P.-A. Malmqvist, B. O. Roos, A. J. Sadlej, and K. Wolinski, J. Phys. Chem. 94, 5483 (1990).

[5] K. Andersson, P.-A. Malmqvist, and B. O. Roos, J. Chem. Phys. 96, 1218 (1992).

[6] G. Ghigo, B. O. Roos, and P.- $\AA$. Malmqvist, Chem. Phys. Lett. 396, 142 (2004).

[7] C. Angeli, R. Cimiraglia, S. Evangelisti, T. Leininger, and J.-P. Malrieu, J. Chem. Phys. 114, 10252 (2001).

[8] C. Angeli, M. Pastore, and R. Cimiraglia, Theor. Chem. Acc. 117, 743 (2007).

[9] K. G. Dyall, J. Chem. Phys. 102, 4909 (1995).

[10] B. O. Roos and K. Andersson, Chem. Phys. Lett. 245, 215 (1995).

[11] N. Forsberg and P.-Å. Malmqvist, Chem. Phys. Lett. 274, 196 (1997).

[12] W. Koch and M. C. Holthausen, A Chemist's Guide to Density Functional Theory (Wiley-VCH, 2000).

[13] P. Hohenberg and W. Kohn, Phys. Rev. 136, B864 (1964).

[14] W. Kohn and L. J. Sham, Phys. Rev. 140, A1133 (1965).

[15] G. E. Scuseria and V. N. Staroverov, in Theory and Applications of Computational Chemistry - The First Forty Years, edited by C. E. Dykstra, G. Frenking, K. S. Kim, and G. E. Scuseria (Elsevier B.V, 2005), pp. 669-724.

[16] J. P. Perdew, K. Burke, and M. Ernzerhof, Phys. Rev. Lett. 77, 3865 (1996).

[17] A. D. Becke, J. Chem. Phys. 98, 1372 (1993). 
[18] A. D. Becke, J. Chem. Phys. 98, 5648 (1993).

[19] P. J. Stephens, F. J. Devlin, C. F. Chabalowski, and M. J. Frisch, J. Phys. Chem. 98, 11623 (1994).

[20] A. D. Becke, Phys. Rev. A 38, 3098 (1988).

[21] C. Lee, W. Yang, and R. G. Parr, Phys. Rev. B 37, 785 (1988).

[22] Y. Zhao and D. G. Truhlar, J. Chem. Phys. 125, 194101 (2006).

[23] Y. Zhao and D. G. Truhlar, J. Phys. Chem. A 110, 13126 (2006).

[24] Y. Zhao and D. Truhlar, Theor. Chem. Acc. 120, 215 (2008).

[25] Y. Zhao and D. G. Truhlar, Acc. Chem. Res. 41, 157 (2008).

[26] K. A. Nguyen, P. N. Day, and R. Pachter, J. Chem. Phys. 135, 074109 (2011).

[27] A. Savin, in Recent Developments and Applications of Modern Density Functional Theory, edited by J. Seminario (Elsevier, 1996), Vol. 4, pp. 327-357.

[28] H. Iikura, T. Tsuneda, T. Yanai, and K. Hirao, J. Chem. Phys. 115, 3540 (2001).

[29] J.-D. Chai and M. Head-Gordon, J. Chem. Phys. 128, 084106 (2008).

[30] T. Yanai, D. P. Tew, and N. C. Handy, Chem. Phys. Lett 393, 51 (2004).

[31] J. P. Perdew and M. Levy, Phys. Rev. B 31, 6264 (1985).

[32] P. W. Ayers and M. Levy, Phys. Rev. A 80, 012508 (2009).

[33] P. W. Ayers, M. Levy, and A. Nagy, Phys. Rev. A 85, 042518 (2012).

[34] M. E. Casida, J. Mol. Struct. 914, 3 (2009).

[35] E. Runge and E. Gross, Phys. Rev. Lett. 52, 997 (1984).

[36] R. van Leeuwen, Phys. Rev. Lett. 82, 3863 (1998).

[37] M. E. Casida, in Recent Advances in Density Functional Methods, Part I, edited by D. P. Chong (World Scientific, 1995), p. 155.

[38] D. J. Tozer, R. D. Amos, N. C. Handy, B. O. Roos, and L. S. Andres, J. Mol. Phys. 97, 859 (1999).

[39] M. E. Casida, F. Gutierrez, J. Guan, F. X. Gadea, and D. Salahub, J. Chem. Phys. 113, 7062 (2000).

[40] A. Dreuw, J. L. Weisman, and M. Head-Gordon, J. Chem. Phys. 119, 2943 (2003). 
[41] A. Lüchow, WIREs Comput Mol Sc 1, 388 (2011).

[42] N. Metropolis, A. Rosenbluth, M. N. Rosenbluth, A. H. Teller, and E. Teller, J. Chem. Phys. 21, 1087 (1953).

[43] C. J. Umrigar, Phys. Rev. Lett. 71, 408 (1993).

[44] C. J. Umrigar, J. Toulouse, C. Filippi, S. Sorella, and R. G. Hennig, Phys. Rev. Lett. 98, 110201 (2007).

[45] C. Filippi, M. Zaccheddu, and F. Buda, J. Chem. Theory Comput. 5, 2074 (2009).

[46] D. M. Ceperley and B. J. Alder, Phys. Rev. Lett. 45, 566 (1980).

[47] H. Lin and D. Truhlar, Theor. Chem. Acc. 117, 185 (2007).

[48] H. M. Senn and W. Thiel, Angew. Chem. Int. Ed. 48, 1198 (2009).

[49] G. Seabra, J. Swails, and A. Roitberg, in Multi-Scale Quantum Models for Biocatalysis, Vol. 7 of Challenges and Advances in Computational Chemistry and Physics, edited by D. York and T.-S. Lee (PUBLISHER, Springer: New York, 2009), pp. 3-20.

[50] B. Mennucci, Phys. Chem. Chem. Phys. 15, 6583 (2013).

[51] J. Tomasi, B. Mennucci, and R. Cammi, Chem. Rev. 105, 2999 (2005).

[52] J. Tomasi and M. Persico, Chem. Rev. 94, 2027 (1994).

[53] V. Barone and M. Cossi, J. Phys. Chem. A 102, 1995 (1998).

[54] D. M. Chipman, J. Chem. Phys. 131, 014103 (2009).

[55] J. Gao, in Reviews in Computational Chemistry (John Wiley \& Sons, Inc., 2007), pp. 119-185.

[56] M. Wanko, M. Hoffmann, P. Strodel, A. Koslowski, W. Thiel, F. Neese, T. Frauenheim, and M. Elstner, J. Phys. Chem. B 109, 3606 (2005).

[57] M. Wanko, M. Hoffmann, T. Frauenheim, and M. Elstner, J. Phys. Chem. B 112, 11462 (2008).

[58] M. Wanko, M. Hoffmann, J. Frähmcke, T. Frauenheim, and M. Elstner, J. Phys. Chem. B 112, 11468 (2008).

[59] R. Send, V. R. I. Kaila, and D. Sundholm, J. Chem. Phys. 134, 214114 (2011).

[60] K. Sneskov, T. Schwabe, O. Christiansen, and J. Kongsted, Phys. Chem. Chem. Phys. 13, 18551 (2011). 
[61] T. Schwabe, J. M. H. Olsen, K. Sneskov, J. Kongsted, and O. Christiansen, J. Chem. Theory Comput. 7, 2209 (2011).

[62] C. Filippi, F. Buda, L. Guidoni, and A. Sinicropi, J. Chem. Theory Comput. 8, $112(2012)$

[63] C. M. Isborn, A. W. Götz, M. A. Clark, R. C. Walker, and T. J. Martínez, J. Chem. Theory Comput. 8, 5092 (2012).

[64] P. Amat and R. Nifosi, J. Chem. Theory Comput. 9, 497 (2013).

[65] T. Wolter, K. Welke, P. Phatak, A.-N. Bondar, and M. Elstner, Phys. Chem. Chem. Phys. 15, 12582 (2013).

[66] O. Valsson, P. Campomanes, I. Tavernelli, U. Rothlisberger, and C. Filippi, J. Chem. Theory Comput. 9, 2441 (2013).

[67] V. R. I. Kaila, R. Send, and D. Sundholm, Phys. Chem. Chem. Phys. 15, 4491 (2013).

[68] T. Schwabe, M. T. P. Beerepoot, J. M. H. Olsen, and J. Kongsted, Phys. Chem. Chem. Phys. 17, (2015).

[69] R. Send, C.-M. Suomivuori, V. R. I. Kaila, and D. Sundholm, J. Phys. Chem. B 119, 2933 (2015).

[70] C. Daday, C. Curutchet, A. Sinicropi, B. Mennucci, and C. Filippi, J. Chem. Theory Comput. 11, 4825 (2015).

[71] M. A. Thompson, J. Phys. Chem. 100, 14492 (1996).

[72] C. Curutchet, A. Muñoz-Losa, S. Monti, J. Kongsted, G. D. Scholes, and B. Mennucci, J. Chem. Theory Comput. 5, 1838 (2009).

[73] J. M. Olsen, K. Aidas, and J. Kongsted, J. Chem. Theory Comput. 6, 3721 (2010).

[74] L. V. Slipchenko, J. Phys. Chem. A 114, 8824 (2010).

[75] C. Amovilli and N. H. March, Chem. Phys. Lett. 347, 459 (2008).

[76] R. Cammi, S. Corni, B. Mennucci, and J. Tomasi, J. Chem. Phys. 122, 104513 (2005).

[77] S. Corni, R. Cammi, B. Mennucci, and J. Tomasi, J. Chem. Phys. 123, 134512 (2005).

[78] F. Aquilante, L. De Vico, N. Ferré, G. Ghigo, P.-Å. Malmqvist, P. Neogrády, T. B. Pedersen, M. Pitoňák, M. Reiher, B. O. Roos, L. Serrano-Andrés, M. Urban, V. Veryazov, and R. Lindh, J. Comput. Chem. 31, 224 (2010). 
[79] M. J. Frisch, G. W. Trucks, H. B. Schlegel, G. E. Scuseria, M. A. Robb, J. R. Cheeseman, G. Scalmani, V. Barone, B. Mennucci, G. A. Petersson, H. Nakatsuji, M. Caricato, X. Li, H. P. Hratchian, A. F. Izmaylov, J. Bloino, G. Zheng, J. L. Sonnenberg, M. Hada, M. Ehara, K. Toyota, R. Fukuda, J. Hasegawa, M. Ishida, T. Nakajima, Y. Honda, O. Kitao, H. Nakai, T. Vreven, J. J. A. Montgomery, J. E. Peralta, F. Ogliaro, M. Bearpark, J. J. Heyd, E. Brothers, K. N. Kudin, V. N. Staroverov, R. Kobayashi, J. Normand, K. Raghavachari, A. Rendell, J. C. Burant, S. S. Iyengar, J. Tomasi, M. Cossi, N. Rega, J. M. Millam, M. Klene, J. E. Knox, J. B. Cross, V. Bakken, C. Adamo, J. Jaramillo, R. Gomperts, R. E. Stratmann, O. Yazyev, A. J. Austin, R. Cammi, C. Pomelli, J. W. Ochterski, R. L. Martin, K. Morokuma, V. G. Zakrzewski, G. A. Voth, P. Salvador, J. J. Dannenberg, S. Dapprich, A. D. Daniels, Ö. Farkas, J. B. Foresman, J. V. Ortiz, J. Cioslowski, and D. J. Fox, Gaussian 09 Revision A.02, Gaussian Inc. Wallingford CT 2009.

[80] M. W. Schmidt, K. K. Baldridge, J. A. Boatz, S. T. Elbert, M. S. Gordon, J. H. Jensen, S. Koseki, N. Matsunaga, K. A. Nguyen, S. Su, T. L. Windus, M. Dupuis, and J. A. M. Jr, J. Comput. Chem. 14, 1347 (1993).

[81] CFOUR, Coupled-Cluster techniques for Computational Chemistry, a quantum-chemical program package by J.F. Stanton, J. Gauss, M.E. Harding, P.G. Szalay with contributions from A.A. Auer, R.J. Bartlett, U. Benedikt, C. Berger, D.E. Bernholdt, Y.J. Bomble, L. Cheng, O. Christiansen, M. Heckert, O. Heun, C. Huber, T.-C. Jagau, D. Jonsson, J. Jusélius, K. Klein, W.J. Lauderdale, D.A. Matthews, T. Metzroth, L.A. Mück, D.P. O’Neill, D.R. Price, E. Prochnow, C. Puzzarini, K. Ruud, F. Schiffmann, W. Schwalbach, S. Stopkowicz, A. Tajti, J. Vázquez, F. Wang, J.D. Watts and the integral packages MOLECULE (J. Almlöf and P.R. Taylor), PROPS (P.R. Taylor), ABACUS (T. Helgaker, H.J. Aa. Jensen, P. Jørgensen, and J. Olsen), and ECP routines by A. V. Mitin and C. van Wüllen. For the current version, see http://www.cfour.de. (accessed July 12, 2012).

[82] Neese, F. ORCA - an ab initio, Density Functional and Semiempirical program package, Version 2.8. Max-Planck-Insitut für Bioanorganische Chemie, Mülheim an der Ruhr, 2011. 


\section{Chapter 3}

\section{Ground- and excited-state geometry optimization of small organic molecules with quantum Monte Carlo ${ }^{\dagger}$}

We present a comparative study of the geometry optimization in the gas phase of acrolein, acetone, methylenecyclopropene, and the propenoic acid anion with special emphasis on their excited-state structures, using quantum Monte Carlo (QMC), multireference perturbation theory (CASPT2 and NEVPT2), second-order approximate coupled cluster (CC2), and time-dependent density functional theory (TDDFT). We find that, for all molecules, the geometries optimized with QMC in its simplest variational (VMC) flavor are in very good agreement with the perturbation results both in the ground and the excited states of either $n \rightarrow \pi^{*}$ or $\pi \rightarrow \pi^{*}$ character. Furthermore, the quality of the QMC structures is superior to those obtained with the CC2 method, which overestimates the $\mathrm{CO}$ bond in all $n \rightarrow \pi^{*}$ excitations, or to the symmetryadapted-cluster configuration interaction (SAC-CI) approach, which gives a poorer description of the CC bonds in the excited states. Finally, the spread in the TDDFT structures obtained with several current exchange-correlation functionals is large and does not reveal a clear relation between the defining features of the functionals and the quality of the optimized structures. In summary, our findings demonstrate the good performance of QMC in optimizing the geometries of these molecules, also in cases where other correlated or TDDFT approaches are inaccurate, and indicate that the method represents a robust reference approach for future structural studies also of larger systems.

$\dagger$ This chapter has been published as R. Guareschi and C. Filippi, "Ground- and excited-state geometry optimization of small organic molecules with quantum Monte Carlo", J. Chem. Theory Comput. 2013, 9, 5513-5525 


\subsection{Introduction}

The optimization of ground- and excited-state structures of molecular systems is an area of fundamental importance in the application of quantum chemical methods. While it is possible to choose among a variety of first-principle approaches, these often display different performances as regards the computational cost or the accuracy of the resulting structures, especially if one considers geometrical relaxation in an electronically excited state.

Time-dependent density functional theory (TDDFT) is the most efficient approach for the computation of excited states of large molecules [1-3] but relies in practice on the choice of an approximate exchange-correlation functional, which may severely limit its accuracy, in particular in describing excitations characterized by charge transfer or multi-reference character $[4,5]$. Therefore, significant research effort has been devoted to develop novel functionals, the long-range corrected being just one example [6-13], and to benchmark the performance of the numerous available approximations [14-25]. Benchmarking has mostly focused on the quality of the vertical excitation energies $[16-18,21]$ but several studies have recently attempted to also establish the performance of TDDFT in describing the optimal excited-state structures and fluorescence $[14,20,23,24]$ also with the inclusion of a solvent via the polarizable continuum model $[15,19,22,25]$. While these investigations concur on the fact that the spread of TDDFT geometries obtained with different exchangecorrelation functionals can be rather large, the assessment of the relative accuracy of these structures is hindered by the corresponding spread of reference geometries obtained at a higher level of theory.

The complete-active-space second-order perturbation theory (CASPT2), symmetry-adapted-cluster configuration interaction (SAC-CI), and second-order approximate coupled-cluster (CC2) have been adopted as reference in different TDDFT studies $[19,23,25]$ but these approaches may yield significantly different equilibrium geometries in the excited state of some particular molecule. For instance, CC2 generally overestimates the CO bond lengths in the $n \rightarrow \pi^{*}$ excited states $[25,26]$ and the selection of this reference method can clearly introduce a bias in the evaluation of the quality of a TDDFT geometrical optimization.

Quantum Monte Carlo (QMC) methods represent an alternative class of highlycorrelated approaches which solve the Schrödinger equation by stochastic means, enabling the use of accurate and compact many-body wave functions with an explicit dependence on the inter-particle distances [27-29]. While the most common variational (VMC) and diffusion (DMC) Monte Carlo variants have been routinely employed for the study of ground-state properties of molecules and solids, their use for the investigation of excited states mainly dates to the last few years [30-41] and has been primarily restricted to the computation of vertical excitation energies. Importantly, these approaches have been shown to yield excitation energies for several prototypical photosensitive molecules [36-41] in good agreement with multi-reference perturbation approaches as CASPT2 in its most recent IPEA formulation [42] and the $n$-electron valence perturbation theory (NEVPT2) [43]. Geometry optimization [37,44-47] is also possible within QMC thanks to recent methodological ad- 
vances, in particular, the robust optimization of wave functions within VMC in a state-specific [48] and state-average [36] fashion, and the computation of interatomic forces with a finite-variance estimator [44] and efficient analytic expressions [45]. To date, the only attempt to optimize the geometry of a non-trivial excited state via QMC gradients remains however our work on retinal chromophore models [37].

In this paper, we present a detailed QMC study of the excited-state structures (stationary points and global minima) obtained for a set of small organic molecules in the gas phase ( $s$-cis and $s$-trans acrolein, acetone, methylenecyclopropene, and the propenoic acid anion) often used to assess the accuracy of TDDFT or other approaches. The aim of this paper is twofold. Since QMC has not been often used to optimize the geometry of excited states, we want to demonstrate here the good quality of VMC geometries as well as offer a comparison with other popular methods often used in benchmark studies. To this purpose, we revisit the optimization of the structures within CASPT2 (and within NEVPT2 for some of these molecules), paying particular attention to the choice of the active space used in the reference wave function. This can in fact significantly affect the resulting optimal geometry as in the case of the propenoic acid anion, where we obtain a different equilibrium structure than in previous CASPT2 studies [49]. In addition to these perturbation approaches, we also compare our QMC results with the structures we obtain with $\mathrm{CC} 2$ and a variety of present-day TDDFT functionals, as well as with SAC-CI results available in the literature [50]. Our comparative study demonstrates that the use of VMC interatomic gradients in combination with fully optimized wave functions yields very accurate excited-state structures, and that VMC represents a robust reference theory for future benchmark studies.

In the Section 3.3, we present the computational details and, in Section 3.2, discuss various methodological aspects regarding the wave functions and the computation of interatomic gradients in QMC. In Section 3.4, we present a detailed analysis of our results and conclude in Section 3.5.

\subsection{Methods}

QMC methods provide an accurate and balanced description of dynamical and static electronic correlation in molecular systems for the computation of ground- and excited-state properties. As already explained in Chapter 2, the most commonly used QMC wave functions are of the so-called Jastrow-Slater type, namely, a linear combination of spin-adapted configuration state functions (CSFs) multiplied by a Jastrow correlation factor, $\mathcal{J}$ :

$$
\Psi_{I}=\mathcal{J} \sum_{i=1}^{N_{\mathrm{CSF}}} c_{i} C_{i},
$$

where $c_{i}$ are the expansion coefficients of the CSFs, $C_{i}$, for the state of interest, $I$. The Jastrow factor depends explicitly on the inter-particle distances and we consider here different Jastrow forms either including only two-body interactions $\left(\mathcal{J}_{2 \text {-body }}\right)$ to account for electron-nuclear and electron-electron correlations, or also also three- 
body terms to describe electron-electron-nuclear correlations $\left(\mathcal{J}_{3-\text { body }}\right)$ [51]. Different Jastrow factors are used to describe different atom types.

The linear coefficients, the orbitals, and the Jastrow parameters are optimized by energy minimization within VMC in a state-specific [48] or state-average [36] fashion. If we are interested in the lowest state of a given symmetry, we simply minimize the corresponding variational energy with respect to the wave function parameters. In the case of a not-trivial excited state with lower-energy states of the same symmetry, we minimize the weighted-averaged energy over the states under consideration:

$$
E_{\mathrm{SA}}=\sum_{I}^{\text {Nstates }} w_{I} \frac{\left\langle\Psi_{I}|\mathcal{H}| \Psi_{I}\right\rangle}{\left\langle\Psi_{I} \mid \Psi_{I}\right\rangle},
$$

where the weights sum to one. The wave functions $\Psi_{I}$ of the different states share a common set of single-particle orbitals and a common Jastrow factor but depend on their individual linear coefficients $c_{i}^{I}$, which are determined to ensure orthogonality between the states. At convergence, the averaged energy is stationary with respect to all parameter variations subject to the orthogonality constraint, while the energies of the individual states are stationary with respect to variations of the linear coefficients but not with respect to variations of the orbital or Jastrow parameters. In the case of multiple states, the expectation values of the quantities needed for the optimization of the parameters are computed in a single VMC run by sampling the square of a guiding wave function, $\Psi_{g}^{2}$, which is here chosen equal to $\sum_{I}\left|\Psi_{I}\right|^{2}$.

To obtain the interatomic forces for a given wave function $\Psi$ for either the ground or the excited state, we need to differentiate the VMC energy which is computed as

$$
E_{\mathrm{V}}=\int \rho(\mathbf{R}) E_{\mathrm{L}}(\mathbf{R}) d \mathbf{R}
$$

where $\mathbf{R}$ denotes the electronic coordinates, $E_{\mathrm{L}}(\mathbf{R})=\mathcal{H} \Psi(\mathbf{R}) / \Psi(\mathbf{R})$ is the local energy, and $\rho(\mathbf{R})=|\Psi(\mathbf{R})|^{2} /\langle\Psi(\mathbf{R}) \mid \Psi(\mathbf{R})\rangle$ is the probability distribution sampled in the Monte Carlo run. The force is then given by

$$
\mathbf{F}_{\alpha}=-\nabla_{\alpha} E_{\mathrm{V}}=-\int \rho(\mathbf{R})\left[\nabla_{\alpha} E_{\mathrm{L}}(\mathbf{R})+\left(E_{\mathrm{L}}(\mathbf{R})-E_{\mathrm{V}}\right) \nabla_{\alpha} \ln \rho(\mathbf{R})\right] d \mathbf{R},
$$

where the gradient is computed with respect to the nuclear coordinate of atom $\alpha$. This expression of the force is equivalent to the more familiar one obtained by using the Hermiticity of the Hamiltonian, where the first term is substituted with the HellmannFeynman force. The reason for using this estimator of the energy gradient is that it obeys a zero-variance principle: If both the trial wave function and its gradient approach the exact ones, the estimator has zero variance $\left(E_{\mathrm{L}}\right.$ and $\nabla_{\alpha} E_{\mathrm{L}}$ become constant and the second term in Eq. 3.4 is identically zero). Consequently, in this limit, one Monte Carlo step is sufficient to sample this expectation value.

In practice, $\Psi(\mathbf{R})$ is an approximate wave function and, even though the estimator has zero variance in the limit of an exact wave function, it has in general infinite variance for an approximate one. Both $E_{\mathrm{L}}$ and $\nabla_{\alpha} \ln \rho$ diverge at the nodes of $\Psi(\mathbf{R})$ as $1 / d$ where $d$ is the distance from the nodal region, so their product diverges as 
$1 / d^{2}$. Since the distribution $\rho$ goes to zero as $d^{2}$, this leads to a finite estimator of the force but to an infinite variance (the integral of $d^{2} \times\left(1 / d^{2}\right)^{2}$ is unbounded). This results in undesirable large spikes in the forces and affects the geometry optimization procedure slowing down the convergence of the calculation. Fortunately, there is a simple solution to obtain a stable estimate of the interatomic forces which consists in sampling a modified distribution $\tilde{\rho}$ corresponding to a wave function, $\tilde{\Psi}(\mathbf{R})$, equal to $\Psi(\mathbf{R})$ in the region far from the nodes but finite at the nodes. In the averages, the product of $E_{\mathrm{L}}$ and $\nabla_{\alpha} \ln \rho$ must then be reweighted with the factor $[\Psi(\mathbf{R}) / \tilde{\Psi}(\mathbf{R})]^{2}$ and has a finite variance [44]. We note that the divergence of the electron-nucleus potential in the term $\nabla_{\alpha} E_{\mathrm{L}}$ also leads to an infinite variance of the force estimator, which can in principle be cured but is here not an issue since we are using pseudopotentials.

So far, we have neglected the implicit dependence of the energy on the nuclear coordinates through the variational parameters of the wave function, $\lambda_{i}$. The gradient contains in fact two terms:

$$
F_{\alpha}=-\frac{\partial E_{\mathrm{V}}}{\partial R_{\alpha}}-\sum_{i} \frac{\partial E_{\mathrm{V}}}{\partial \lambda_{i}} \frac{d \lambda_{i}}{d R_{\alpha}}
$$

where the second term is equal to zero for a fully-optimized wave function in energy minimization since $\partial E_{\mathrm{V}} / \partial \lambda_{i}=0$. In the case of a state-average optimized wave function, the energy is optimal with respect to the linear coefficients but not with respect to the orbital and Jastrow parameters, which are obtained by minimizing the average energy. This leads to a small error in the computation of the forces, whose magnitude can be assessed by employing wave functions of increasing quality. Finally, we note that recent developments have made possible the efficient and practical computation of forces within diffusion Monte Carlo [52] but, in the present work, we limit our study to VMC.

The VMC geometry optimizations are performed in Z-matrix coordinates with the use of numerical gradients of the energy with respect to the atomic coordinates. We stress that it is now possible to compute the analytical expression of the forces with a computational cost larger than that of the energy by a modest factor which is independent of the system size [53]. At the time of these calculations, however, analytical forces were not yet implemented in our QMC code and the interatomic forces at a reference geometry were therefore computed in a correlated sampling VMC calculation [54], where a set of secondary geometries is generated through forward and backward displacements of 0.001 a.u. in the bond lengths and $0.01^{\circ}$ in the bond and dihedral angles. The procedure followed here is described in greater detail in our earlier work on structural optimization in VMC [37] and can be summarized in the following steps: $(i)$ The Jastrow parameters, linear coefficients, and the orbitals of the starting wave function are optimized in energy minimization at the initial geometry; (ii) the energy gradients are computed in a correlated sampling VMC calculation; (iii) the geometry is updated following the descendant direction of the energy gradient in an approximate Newton-Raphson method which uses the diagonal elements of the Hessian; (iv) at the new geometry, the wave function is optimized starting form the optimal wave function at the previous step, recentered at the new geometry. 
The procedure is iterated until convergence, namely, until the forces are smaller than 0.001-0.003 Hartree/Bohr for the bond lengths and 0.0001 Hartree/deg for the bond angles. As starting geometries in the ground-state optimizations, we usually employ a DFT structure and, in the excited-state relaxation, an excited-state geometry obtained with another correlated theory. For the more problematic case of the $\pi \rightarrow \pi^{*}$ state of planar acrolein, we test the use of the ground-state VMC geometry as initial guess, and obtain the same final configuration. All VMC structural optimizations converge with less than 10 iterations.

Due to the stochastic nature of VMC, it is not possible to identify a particular geometry as the minimum one. Therefore, after convergence, we perform 5-10 more iterations and obtain the final structure by averaging the internal coordinates over these additional steps. For the geometry optimization of an excited state which is not the lowest in its symmetry class, the wave function parameters are optimized in a state-average manner as described above, but the forces are computed for the root of interest. We note that analytic calculation of VMC forces is possible to reduce the computational cost for systems larger than the ones studied here [45]. Finally, approximate DMC forces [54] could also be employed but their use in combination with fully optimized wave functions does not seem to improve the VMC estimates [37,47], while being significantly more expensive.

\subsection{Computational details}

We perform the TDDFT calculations with the Gaussian09 program [55] and employ a set of exchange-correlation functionals that are representative of different classes of approximations. In particular, we use BLYP $[56,57]$ as local functional with no Hartree-Fock (HF) exchange, B3LYP [58, 59], PBE0 [60-62], M06 [63], and M062X [63] as global hybrids with a fixed amount of HF exchange, and CAM-B3LYP [6] and LC-BLYP [7] for the long-range corrected functionals with a variable percentage of HF exchange which depends on the inter-electronic distance.

We carry out the CC2 [64-67] calculations with the CFOUR code [68] and use the MOLCAS 7.2 suite of programs [69] for the CASPT2 [70,71] calculations, where we always employ the default zero-order IPEA Hamiltonian [42]. The stronglycontracted (SC) NEVPT2 $[43,72,73]$ calculations are performed with the ORCA 2.8 code [74]. With this code, one can exploit symmetry in the single-point calculations but not in the geometry optimization with numerical gradients at the NEVPT2 level. Furthermore, ORCA employs the weighted average of the interatomic gradients to optimize with NEVPT2 the structure of an excited state resulting from a state-average calculation. Therefore, we only employ NEVPT2 to optimize the geometry in the ground state and in the lowest-energy state with a different symmetry than the ground state using a fictitious state-average calculation over two roots with $(0,1)$ weights. This procedure is however only successful for the $n \rightarrow \pi^{*}$ excited state of acrolein.

The program package CHAMP is used for the QMC calculations [75]. We employ scalar-relativistic energy-consistent Hartree-Fock pseudopotentials [76, 77] and obtain the starting determinantal component of the Jastrow-Slater wave function in 
complete-active-space self-consistent field (CASSCF) calculations performed with the program GAMESS(US) [78]. The CAS expansions are then expressed on the CASSCF natural orbitals and all configuration state functions are retained in the QMC studies. Only for the propenoic acid anion, the expansion is truncated with a threshold of 0.05 on the CSF coefficients for subsequent use in the QMC calculations. All parameters in the QMC wave functions are optimized in variational Monte Carlo using the linear method [48] and its extension for state-average calculations [36]. Further details on the QMC calculations are given in the Method section.

The basis set dependence of the geometrical parameters is studied at the TDDFT, CASPT2, NEVPT2, and CC2 level using the Dunning's correlation consistent ccpVXZ and aug-cc-pVXZ basis sets [79-82]. In the QMC calculations, we use the Gaussian basis sets [76] specifically constructed for our pseudopotentials. In particular, we employ the cc-pVDZ and cc-pVTZ basis sets and the corresponding basis augmented with diffuse functions [83] on the heavy atoms. We also use the ccpVTZ' basis set which consist of the cc-pVTZ for the heavy atoms combined with the cc-pVDZ for hydrogen. We show below that the geometrical parameters computed with the TDDFT, CC2, and perturbation methods are well converged with the cc-pVTZ basis set and, within QMC, with the cc-pVTZ' basis.

\subsection{Results}

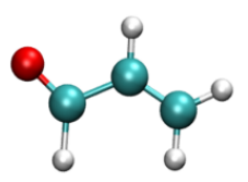

A

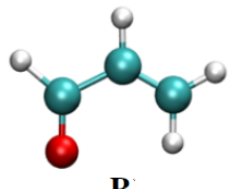

B

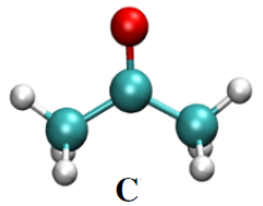

C
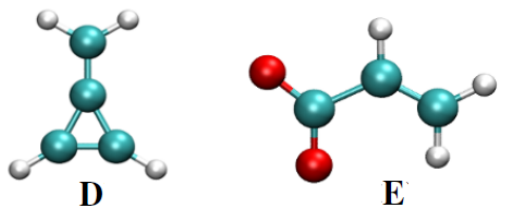

Figure 3.1: The molecules considered in this work: A) $s$-trans and B) $s$-cis acrolein, C) acetone, D) methylenecyclopropene (MCP), and E) the propenoic acid anion (PAA).

The molecules investigated here are the $s$-trans and $s$-cis acrolein, acetone, methylenecyclopropene (MCP), and the propenoic acid anion (PAA), which are shown in Figure 3.1. This set of representative molecules has often been chosen in the literature to study the performance of quantum chemical methods in determining optimal excited-state structures $[19,25,49,50,84-91]$. We consider the structural optimization in the ground state of all molecules, in the $n \rightarrow \pi^{*}$ state of acrolein, acetone, and PAA, and in the $\pi \rightarrow \pi^{*}$ state of acrolein and MCP.

The geometries of these molecules are optimized with different symmetry constraints in their ground and low-lying singlet excited states as detailed in Table 3.1. When planarity is imposed in the excited state, the optimal structures do not correspond to a minimum in the case of acrolein and MCP in the $\pi \rightarrow \pi^{*}$ state, and of acetone in the $n \rightarrow \pi^{*}$ state. For acetone, we also determine the minimum geometry for the $n \rightarrow \pi^{*}$ state, allowing the pyramidalization of the central $\mathrm{C}$ atom, which 
leads to a symmetry reduction from $C_{2 v}$ to $C_{s}$. The out-of-plane optimization is also performed for $s$-trans acrolein in the $\pi \rightarrow \pi^{*}$ excited state, where a twisting around the terminal $\mathrm{C}-\mathrm{CH}_{2}$ bond is expected to bring the system to the minimum energy geometry with a diradical ground state.

\begin{tabular}{lccc}
\hline Molecule & Symmetry & State & Excitation \\
\hline$s$-trans acrolein & $C_{s}$ planar & $1^{1} \mathrm{~A}^{\prime}$ & $\mathrm{GS}$ \\
& & $1^{1} \mathrm{~A}^{\prime \prime}$ & $n \rightarrow \pi^{*}$ \\
& & $2^{1} \mathrm{~A}^{\prime}$ & $\pi \rightarrow \pi^{*}$ \\
& $C_{s}$ twisted & $1^{1} \mathrm{~A}^{\prime \prime}$ & $\mathrm{GS}$ \\
s-cis acrolein & $C_{s}$ planar & $1^{1} \mathrm{~A}^{\prime}$ & $\mathrm{GS}$ \\
& & $1^{1} \mathrm{~A}^{\prime \prime}$ & $n \rightarrow \pi^{*}$ \\
Acetone & $C_{2 v}$ planar & $1^{1} \mathrm{~A}_{1}$ & $\mathrm{GS}$ \\
& & $1^{1} \mathrm{~A}_{2}$ & $n \rightarrow \pi^{*}$ \\
& $C_{s}$ bended & $1^{1} \mathrm{~A}^{\prime \prime}$ & $n \rightarrow \pi^{*}$ \\
MCP & $C_{2 v}$ planar & $1^{1} \mathrm{~A}_{1}$ & $\mathrm{GS}$ \\
& & $1^{1} \mathrm{~B}_{2}$ & $\pi \rightarrow \pi^{*}$ \\
PAA & $C_{s}$ planar & $1^{1} \mathrm{~A}^{\prime}$ & $\mathrm{GS}$ \\
& & $1^{1} \mathrm{~A}^{\prime \prime}$ & $n \rightarrow \pi^{*}$ \\
\hline
\end{tabular}

Table 3.1: Symmetries imposed in the structural optimization of the ground and lowlying excited states of the molecules. The symmetry of the state and the nature of the excitation are also reported. We denote with GS the lowest-energy state for the given geometry.

For these states and molecules, we compute the optimal geometries within VMC, CC2, CASPT2, and NEVPT2. We assess the relative performance of these highlycorrelated methods and compare our results to the geometries obtained with different approximate exchange-correlation functionals within TDDFT. Before presenting the results of our geometry optimizations, we first discuss the dependence of the geometrical parameters obtained with the various methods on the choice of basis set and, in the VMC calculations, on the sophistication of the Jastrow factor. To illustrate the dependence of the methods on these computational ingredients, we focus on for $s$-trans acrolein in the ground and the $n \rightarrow \pi^{*}$ excited state.

\subsubsection{Geometry dependence on basis set}

For $s$-trans acrolein, we employ the cc-pVXZ and aug-cc-pVXZ series to investigate the basis set dependence of the optimal structures computed at the CASPT2, NEVPT2, CC2, and TDDFT level in the ground and the lowest $n \rightarrow \pi^{*}$ excited state.

We report the CASPT2 ground- and excited-state geometries obtained with the cc-pVXZ basis sets in Table 3.2. The enlargement of the basis set from double to quadruple $\zeta$ produces a slight shortening of the bond lengths, which are well converged when the cc-pVTZ basis is employed. The cc-pVDZ bond lengths differ from the cc-pVQZ values by at most $0.017 \AA$ and the use of cc-pVTZ improves the 
agreement to better than $0.005 \AA$. The effect of the augmentation is tested only for the excited state where it might be expected to have an effect. As shown in Figure 3.2, we find instead that the aug-cc-pVXZ series does not performs better than the cc-pVXZ sets since, for the smallest double $\zeta$ basis, it leads to geometries further away from the converged values. As shown in Table 3.3 and Table 3.4, the cc-pVTZ basis leads to well converged geometries in the ground and excited states also at the NEVPT2 and CC2 level.

\begin{tabular}{lccc}
\hline CASPT2 & cc-pVDZ & cc-pVTZ & cc-pVQZ \\
\hline & & $1^{1} \mathrm{~A}^{\prime}$ \\
$\mathrm{C}=\mathrm{O}$ & 1.220 & 1.214 & 1.211 \\
$\mathrm{C}-\mathrm{C}$ & 1.482 & 1.470 & 1.468 \\
$\mathrm{C}=\mathrm{C}$ & 1.352 & 1.339 & 1.337 \\
$\theta(\mathrm{C}-\mathrm{C}-\mathrm{C})$ & 120.78 & 120.36 & 120.25 \\
$\theta(\mathrm{C}-\mathrm{C}-\mathrm{O})$ & 124.02 & 124.16 & 122.67 \\
& & $1^{1} \mathrm{~A}^{\prime \prime}$ & \\
$\mathrm{C}=\mathrm{O}$ & 1.342 & 1.336 & 1.332 \\
$\mathrm{C}-\mathrm{C}$ & 1.388 & 1.377 & 1.375 \\
$\mathrm{C}=\mathrm{C}$ & 1.406 & 1.392 & 1.389 \\
$\theta(\mathrm{C}-\mathrm{C}-\mathrm{C})$ & 123.36 & 123.26 & 123.26 \\
$\theta(\mathrm{C}-\mathrm{C}-\mathrm{O})$ & 124.93 & 124.54 & 124.36 \\
\hline
\end{tabular}

Table 3.2: CASPT2 bond lengths $(\AA)$ and angles (deg) of $s$-trans acrolein in the ground $\left(1^{1} \mathrm{~A}^{\prime}\right)$ and $n \rightarrow \pi^{*}$ excited $\left(1^{1} \mathrm{~A}^{\prime \prime}\right)$ states computed with the cc-pVXZ basis sets.

\begin{tabular}{lccc}
\hline NEVPT2 & cc-pVDZ & cc-pVTZ & cc-pVQZ \\
\hline & & $1^{1} \mathrm{~A}^{\prime}$ & \\
$\mathrm{C}=\mathrm{O}$ & 1.219 & 1.210 & 1.209 \\
$\mathrm{C}-\mathrm{C}$ & 1.479 & 1.462 & 1.458 \\
$\mathrm{C}=\mathrm{C}$ & 1.350 & 1.333 & 1.329 \\
$\theta(\mathrm{C}-\mathrm{C}-\mathrm{C})$ & 120.72 & 120.33 & 119.66 \\
$\theta(\mathrm{C}-\mathrm{C}-\mathrm{O})$ & 124.19 & 124.45 & 123.90 \\
& & $1^{1} \mathrm{~A}^{\prime \prime}$ & \\
$\mathrm{C}=\mathrm{O}$ & 1.339 & 1.328 & 1.326 \\
$\mathrm{C}-\mathrm{C}$ & 1.387 & 1.371 & 1.371 \\
$\mathrm{C}=\mathrm{C}$ & 1.403 & 1.385 & 1.383 \\
$\theta(\mathrm{C}-\mathrm{C}-\mathrm{C})$ & 123.40 & 123.37 & 123.42 \\
$\theta(\mathrm{C}-\mathrm{C}-\mathrm{O}$ & 125.00 & 124.79 & 124.39 \\
\hline
\end{tabular}

Table 3.3: NEVPT2 bond lengths $(\AA)$ and angles (deg) of $s$-trans acrolein in the the ground $\left(1^{1} \mathrm{~A}^{\prime}\right)$ and $n \rightarrow \pi^{*}$ excited $\left(1^{1} \mathrm{~A}^{\prime \prime}\right)$ states computed with different basis sets.

To investigate the basis set dependence of the TDDFT geometries, we select the CAM-B3LYP functional since the convergence is rather similar for the differ- 


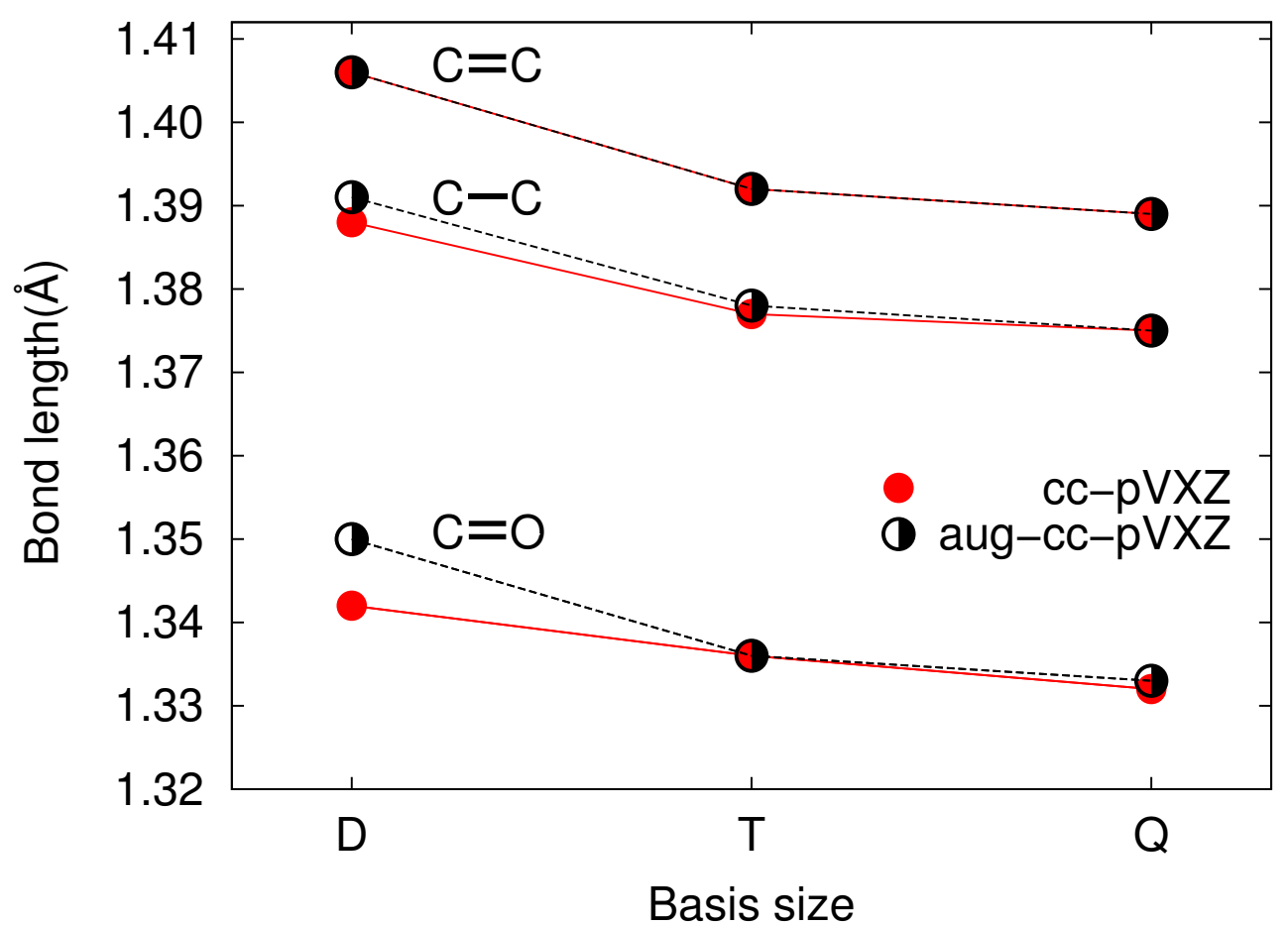

Figure 3.2: Convergence of the bond lengths ( $\AA$ ) of $s$-trans acrolein in the $n \rightarrow \pi^{*}$ excited state computed at the CASPT2 level with the cc-pVXZ basis sets.

ent functionals. The dependence of the bond lengths in the excited state are shown in Figure 3.3 and is rather similar to the one observed for the perturbation and CC2 methods, with bond lengths which are well converged when the cc-pVTZ basis is employed. The aug-cc-pVXZ series yields practically equivalent results to the cc-pVXZ sets.

On the basis of these results, we do not employ diffuse functions in the CASPT2, NEVPT2, CC2, and TDDFT geometry optimization of the ground states and of the valence excited states of the molecules investigated here, and use the cc-pVTZ basis set as the default basis to compare the performance of the different methods.

\subsubsection{VMC geometry dependence on basis set and Jastrow factor}

We investigate the dependence of the VMC geometries on the choice of basis set and Jastrow factor in the optimization of the ground and the $n \rightarrow \pi^{*}$ excited state of $s$-trans acrolein. As described in Section 3.3, we use correlated-consistent basis sets specifically constructed for the pseudopotentials we employ in the QMC calculations. Here, we test the double $\zeta$ basis (pVDZ), the triple basis for the heavy atoms combined with a double $\zeta$ for hydrogen $(\mathrm{pVTZ})$, and the triple basis set for all atoms (pVTZ). We also perform calculations augmenting these basis sets with diffuse functions on the heavy atoms. Furthermore, we employ two different Jastrow 


\begin{tabular}{lccc}
\hline $\mathrm{CC} 2$ & cc-pVDZ & cc-pVTZ & cc-pVQZ \\
\hline & & $1^{1} \mathrm{~A}^{\prime}$ & \\
$\mathrm{C}=\mathrm{O}$ & 1.229 & 1.221 & 1.220 \\
$\mathrm{C}-\mathrm{C}$ & 1.481 & 1.463 & 1.462 \\
$\mathrm{C}=\mathrm{C}$ & 1.353 & 1.336 & 1.335 \\
$\theta(\mathrm{C}-\mathrm{C}-\mathrm{C})$ & 120.28 & 120.00 & 119.97 \\
$\theta(\mathrm{C}-\mathrm{C}-\mathrm{O})$ & 124.45 & 124.56 & 124.38 \\
& & $1^{1} \mathrm{~A}^{\prime \prime}$ & \\
$\mathrm{C}=\mathrm{O}$ & 1.369 & 1.368 & 1.371 \\
$\mathrm{C}-\mathrm{C}$ & 1.391 & 1.370 & 1.369 \\
$\mathrm{C}=\mathrm{C}$ & 1.398 & 1.383 & 1.382 \\
$\theta(\mathrm{C}-\mathrm{C}-\mathrm{C})$ & 122.20 & 122.14 & 122.15 \\
$\theta(\mathrm{C}-\mathrm{C}-\mathrm{O})$ & 127.83 & 127.15 & 126.67 \\
\hline
\end{tabular}

Table 3.4: CC2 bond lengths ( $⿱$ A) and angles (deg) of $s$-trans acrolein in the the ground $\left(1^{1} \mathrm{~A}^{\prime}\right)$ and $n \rightarrow \pi^{*}$ excited $\left(1^{1} \mathrm{~A}^{\prime \prime}\right)$ states computed with different basis sets.

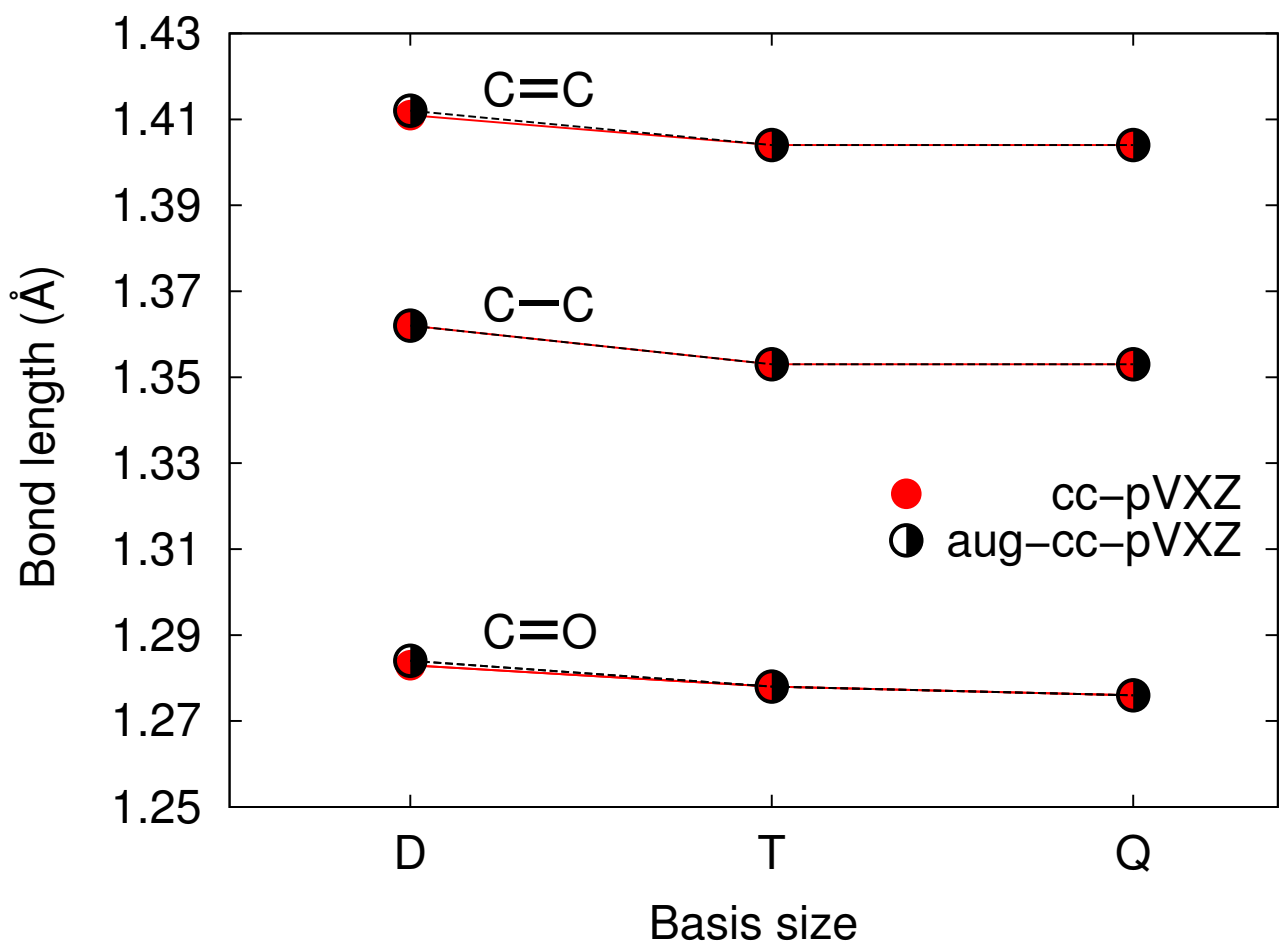

Figure 3.3: Convergence of the bond lengths $(\AA)$ of $s$-trans acrolein in the $n \rightarrow \pi^{*}$ excited state computed within TDDFT/CAM-B3LYP with the cc-pVXZ basis sets.

factors, that is, a two-body Jastrow factor $\left(\mathcal{J}_{2 \text {-body }}\right)$ and a three-body Jastrow factor $\left(\mathcal{J}_{3 \text {-body }}\right)$. We report the geometries optimized with a two-body Jastrow factor in Table 3.5.

In line with what observed for all other methods, we find that the bonds con- 


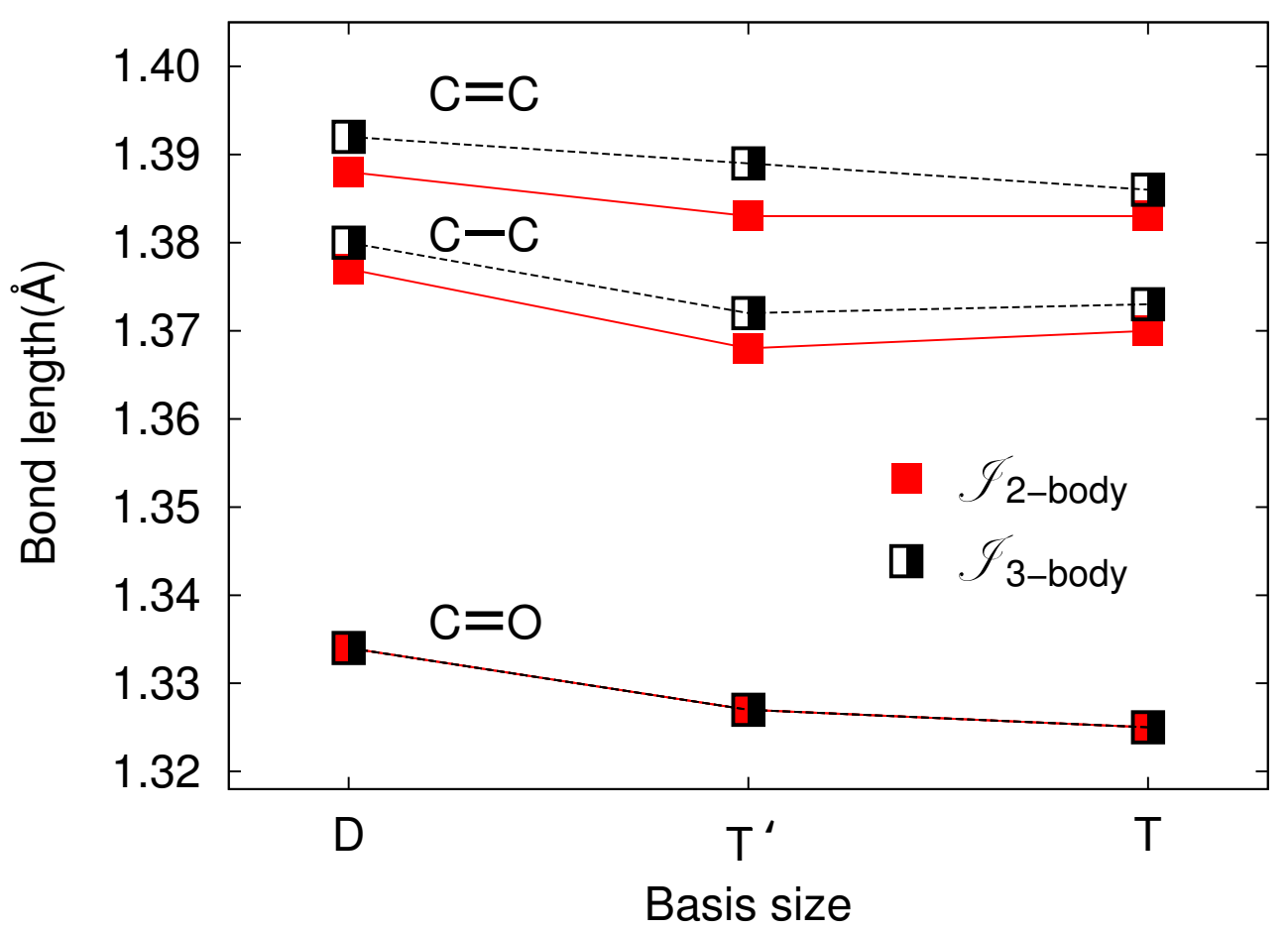

Figure 3.4: Convergence of the VMC bond lengths $(\AA)$ of $s$-trans acrolein in the $n \rightarrow \pi^{*}$ excited state computed with different basis sets and Jastrow factors. The statistical error bars are smaller than the symbol size.

tract when the basis set is enlarged as also shown in Figure 3.4. Furthermore, also in $\mathrm{VMC}$, the inclusion of diffuse functions slows down the convergence of the bond lengths with basis size (not shown in the Table). A difference with the other correlated approaches is that VMC displays a weaker dependence of the geometrical parameters on the basis set thanks to the presence of the Jastrow correlation function. We observe that the use of a valence triple $\zeta$ basis on the heavy atoms and a double on hydrogen ( $\mathrm{pVTZ}$ ') leads to bond lengths which are converged to better than $0.01 \AA$ A. Finally, introducing a three-body Jastrow factor gives a similar convergence with respect to the basis as the two-body Jastrow factor, and leads to equivalent bond lengths within $0.003-0.006 \AA$. Therefore, as a good compromise between accuracy and computational cost, the VMC optimizations will be performed with the $\mathrm{pVTZ}^{\prime}$ basis set and a two-body Jastrow factor for all the other systems, since the use of a three-body Jastrow factor does not appreciably affect the results but significantly increases the time of the simulations.

\subsubsection{Acrolein}

The states of $s$-trans acrolein considered here are the ground state $\left(1^{1} \mathrm{~A}^{\prime}\right)$ and the $n \rightarrow \pi^{*}\left(1^{1} \mathrm{~A}^{\prime \prime}\right)$ and $\pi \rightarrow \pi^{*}$ excited states $\left(2^{1} \mathrm{~A}^{\prime}\right)$. The three states are optimized with planarity constraint $\left(C_{s}\right.$ symmetry). The $2^{1} \mathrm{~A}^{\prime}$ excited state is also studied in his 


\begin{tabular}{lccc}
\hline $\mathrm{VMC}$ & $\mathrm{pVDZ}$ & $\mathrm{pVTZ}^{\prime}$ & $\mathrm{pVTZ}$ \\
\hline & & $1^{1} \mathrm{~A}^{\prime}$ & \\
$\mathrm{C}=\mathrm{O}$ & $1.207(0)$ & $1.205(1)$ & $1.202(1)$ \\
$\mathrm{C}-\mathrm{C}$ & $1.466(0)$ & $1.464(1)$ & $1.460(1)$ \\
$\mathrm{C}=\mathrm{C}$ & $1.333(1)$ & $1.328(1)$ & $1.328(0)$ \\
$\theta(\mathrm{C}-\mathrm{C}-\mathrm{C})$ & $118.93(8)$ & $120.89(6)$ & $120.25(5)$ \\
$\theta(\mathrm{C}-\mathrm{C}-\mathrm{O})$ & $124.86(7)$ & $123.67(4)$ & $124.41(5)$ \\
& & $1^{1} \mathrm{~A}^{\prime \prime}$ & \\
$\mathrm{C}=\mathrm{O}$ & $1.334(0)$ & $1.327(0)$ & $1.325(0)$ \\
$\mathrm{C}-\mathrm{C}$ & $1.377(0)$ & $1.368(0)$ & $1.370(0)$ \\
$\mathrm{C}=\mathrm{C}$ & $1.388(0)$ & $1.383(0)$ & $1.383(0)$ \\
$\theta(\mathrm{C}-\mathrm{C}-\mathrm{C})$ & $123.76(1)$ & $122.57(1)$ & $121.89(0)$ \\
$\theta(\mathrm{C}-\mathrm{C}-\mathrm{O})$ & $123.67(1)$ & $125.82(1)$ & $126.13(0)$ \\
\hline
\end{tabular}

Table 3.5: VMC bond lengths ( $($ ) and angles (deg) of $s$-trans acrolein. We employ different basis sets, specifically constructed for our pseudopotentials, and a two-body Jastrow factor $\left(\mathcal{J}_{2-\text { body }}\right)$. The statistical error on the last digit is given in bracket.

twisted geometry, always maintaining $C_{s}$ symmetry. We also present a brief study of the ground and $n \rightarrow \pi^{*}$ excited-state geometries of the $s$-cis isomer of acrolein. In the following, when speaking about the double or single carbon-carbon bonds of acrolein, we always refer to the ground-state bond structure as depicted in Figure 3.5.<smiles>C=CC=O</smiles><smiles>C=CC=O</smiles>

Figure 3.5: Structures of $s$-trans (left) and $s$-cis (right) acrolein.

\section{Ground-state and $n \rightarrow \pi^{*}$ excited-state geometries of $s$-trans acrolein}

The optimal geometries of $s$-trans acrolein in the ground and $n \rightarrow \pi^{*}$ excited states computed with CC2, CASPT2, NEVPT2, and VMC are given in Table 3.6. In the perturbation and the VMC optimization of the ground and excited states, we use as reference wave function a CAS $(6,5)$ expansion, where the active space comprises four $\pi$ orbitals on the conjugated carbon chain and a $\sigma$ orbital describing a lone pair in the direction orthogonal to the $\mathrm{CO}$ group. Our CASPT2 results for the ground and the $n \rightarrow \pi^{*}$ state are in good agreement with previous benchmark studies of $s$-trans 


\begin{tabular}{lcccc}
\hline & CC2 & CASPT2 & NEVPT2 & VMC \\
\hline & & \multicolumn{3}{c}{$1^{1} \mathrm{~A}^{\prime}$} \\
$\mathrm{C}=\mathrm{O}$ & 1.221 & 1.214 & 1.210 & $1.205(1)$ \\
$\mathrm{C}-\mathrm{C}$ & 1.463 & 1.470 & 1.462 & $1.464(1)$ \\
$\mathrm{C}=\mathrm{C}$ & 1.336 & 1.339 & 1.333 & $1.328(1)$ \\
$\theta(\mathrm{C}-\mathrm{C}-\mathrm{C})$ & 120.00 & 120.36 & 120.33 & $120.89(6)$ \\
$\theta(\mathrm{C}-\mathrm{C}-\mathrm{O})$ & 124.56 & 124.16 & 124.45 & $123.67(4)$ \\
& & \multicolumn{4}{c}{$1^{1} \mathrm{~A}^{\prime \prime}$} \\
$\mathrm{C}=\mathrm{O}$ & 1.368 & 1.336 & 1.328 & $1.327(0)$ \\
$\mathrm{C}-\mathrm{C}$ & 1.370 & 1.377 & 1.371 & $1.368(0)$ \\
$\mathrm{C}=\mathrm{C}$ & 1.383 & 1.392 & 1.385 & $1.383(0)$ \\
$\theta(\mathrm{C}-\mathrm{C}-\mathrm{C})$ & 122.14 & 123.26 & 123.37 & $122.57(1)$ \\
$\theta(\mathrm{C}-\mathrm{C}-\mathrm{O})$ & 127.15 & 124.54 & 124.79 & $125.82(1)$ \\
\hline
\end{tabular}

Table 3.6: CC2, CASPT2, NEVPT2, and VMC bond lengths ( $\AA$ ) and angles (deg) of $s$-trans acrolein in the ground state $\left(1^{1} \mathrm{~A}^{\prime}\right)$ and $n \rightarrow \pi^{*}$ excited states $\left(1^{1} \mathrm{~A}^{\prime \prime}\right)$. We employ a cc-pVTZ basis in the CC2 and PT2 calculations, and a pVTZ' basis set in combination with a two-body Jastrow factor in VMC.

acrolein based on a different CASPT2 approach (with the IPEA shift set to zero in the zero-order Hamiltonian) [49,84].

For the ground state, the optimal $\mathrm{CC} 2$, perturbation, and VMC geometries agree very well. The CASPT2 bond lengths are about $0.005 \AA$ longer than the NEVPT2 values, and the CASPT2 and NEVPT2 results differ from the VMC ones by less than 0.01 and $0.005 \AA$, respectively. The CC2 method displays somewhat larger deviations from the other techniques in the $\mathrm{CO}$ bond, which is $0.015 \AA$ longer than the VMC value. We note that, for the ground state, there exists a second CASSCF minimum very closed in energy and corresponding to a CAS $(6,5)$ expansion which includes a lone-pair orbital parallel to the $\mathrm{CO}$ group. The structural optimization performed at the CASPT2 level with this expansion leads to a geometry which agrees to better than $0.001 \AA$ [92] with the one of Table 3.6, indicating that the inclusion in the active space of either lone pair does not substantially influence the optimal ground-state geometry obtained in the subsequent correlated calculation.

As shown in the upper panel of Figure 3.6, we find a good agreement between the perturbation theories and VMC also in the excited state, with differences of the same order of magnitude as those observed for the ground state. In particular, the NEVPT2 bond lengths are again in excellent agreement with the VMC values, displaying deviations smaller than $0.003 \AA$. The agreement of $\mathrm{CC} 2$ with the other approaches is instead less satisfactory: While CC2 gives $\mathrm{CC}$ bond lengths which are compatible with the VMC values, it overestimates the $\mathrm{CO}$ bond by as much as $0.04 \AA$. This behavior is in line with the general tendency of the method to overestimate the carbonyl bond in the $n \rightarrow \pi^{*}$ states of aldehydes [26]. A comparison with the SAC-CI calculations with the cc-pVTZ basis set of Ref. [50] reveals a poor performance also of the SAC-CI approach, which overestimates the CC bond, formerly single in the 

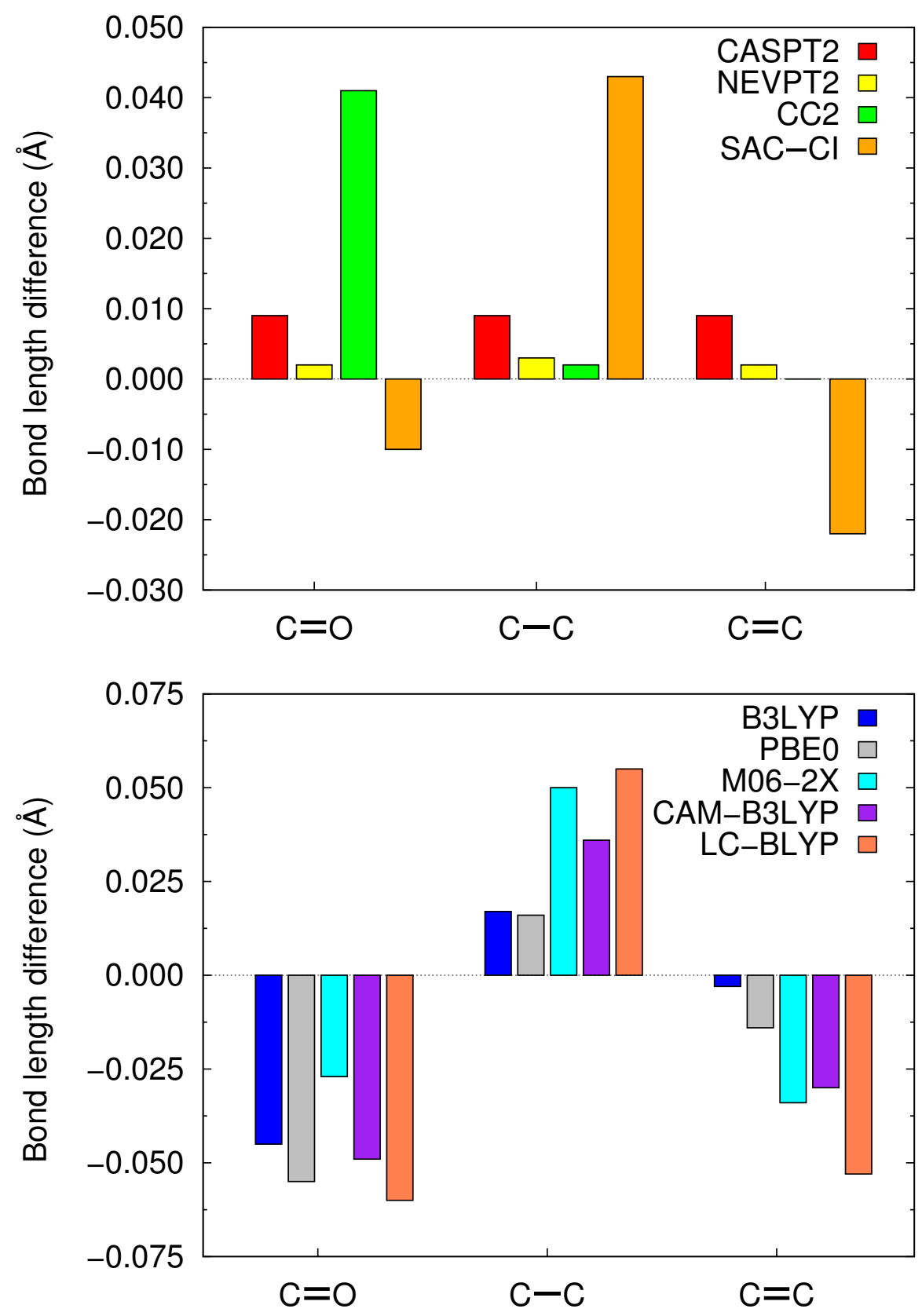

Figure 3.6: Bond-length differences $(\AA)$ between the geometries computed with different methods and the VMC reference for the $n \rightarrow \pi^{*}$ excited state of $s$-trans acrolein. The SAC-CI/cc-pVTZ values are from Ref. [50].

ground state, by $0.045 \AA$ and underestimates the other CC bond by $0.025 \AA$, while the agreement on the $\mathrm{CO}$ bond length is rather good.

In the lower panel of Figure 3.6, we assess the quality of the TDDFT geometries against out VMC data. We employ a set of functionals which comprises some (B3LYP, PBE0, and CAM-B3LYP) considered also in previous benchmark studies $[19,25,84]$. The errors in the TDDFT bond lengths are compatible with those 
reported in Ref. [25], with the noteworthy exception of the $\mathrm{CO}$ bond. The apparent difference for this bond is however simply due to the use of a different reference geometry: The deviation of TDDFT with respect to VMC is about $-0.05 \AA$ for all the functionals considered here, but increases to $-0.1 \AA$ when CC2 with the the aug-ccpVTZ basis set is taken as reference as in Ref. [25]. Considering the near breakdown of the CC2 model in the description of the $n \rightarrow \pi^{*}$ excited state of acrolein (as well as of acetone and the propenoic acid anion) as also discussed in previous studies $[25,26]$, the choice of this method as reference for TDDFT geometry optimizations is surely not appropriate for this class of molecules. As for the performance of the TDDFT functionals, there is not a clear trend when increasing the amount of exact exchange in going from B3LYP (20\%) to M06-2X (54\%). While the use of the M06-2X halves the error on the $\mathrm{CO}$ bond, it significantly increases the deviations on the $\mathrm{CC}$ bonds by not sufficiently shortening/lengthening the bonds formerly single/double in the ground state. The use of the long-range corrected functionals CAM-B3LYP and LC-BLYP with either $65 \%$ and $100 \%$ exchange at long distances leads to significantly larger errors on the $\mathrm{CC}$ bonds and a comparable error on the $\mathrm{CO}$ bond as the hybrid BLYP and PBE0 functionals. We note here that, in all Figures throughout the paper, we employ VMC as our reference. This choice is mainly motivated by the fact that the NEVPT2 results are only available for acrolein, where they agree remarkably well with VMC, while the use of CASPT2 as reference poses a problem for the $\pi \rightarrow \pi^{*}$ state of acrolein as discussed below.

\section{Ground-state and $n \rightarrow \pi^{*}$ excited-state geometries of $s$-cis acrolein}

In the literature, the cis configuration instead of the more stable trans configuration of acrolein (see Figure 3.5) has sometimes been adopted to benchmark the electronic excitations of various methods such as TDDFT [19] and CASPT2 [84]. We expect that all considerations above about $s$-trans acrolein are directly transferable also to $s$-cis acrolein and, to demonstrate this, we optimize here the ground state and the $n \rightarrow \pi^{*}$ state of this isomer with the methods also used for $s$-trans acrolein.

The ground-state geometries display an excellent agreement and, for the $n \rightarrow \pi^{*}$ excited state, we observe the same behavior noted for the trans isomer as shown in Figure 3.7 (upper panel): While the agreement is very good between the perturbation theories and the VMC method, CC2 significantly overestimates the $\mathrm{CO}$ bond. The $\mathrm{CC} 2$ error on the $\mathrm{CO}$ bond is in fact somewhat larger than for the trans isomer.

For the functionals selected to test TDDFT, the errors with respect to the VMC optimized structure are of the same order of magnitude as for trans acrolein (see lower panel of Figure 3.7). Again, we observe a satisfactory performance of B3LYP and $\mathrm{PBE} 0$ for the $\mathrm{CC}$ bonds, while the $\mathrm{CO}$ bond length is underestimated by as much as $0.05 \AA$. The error on this bond is in the same range also for the other functionals considered here. 

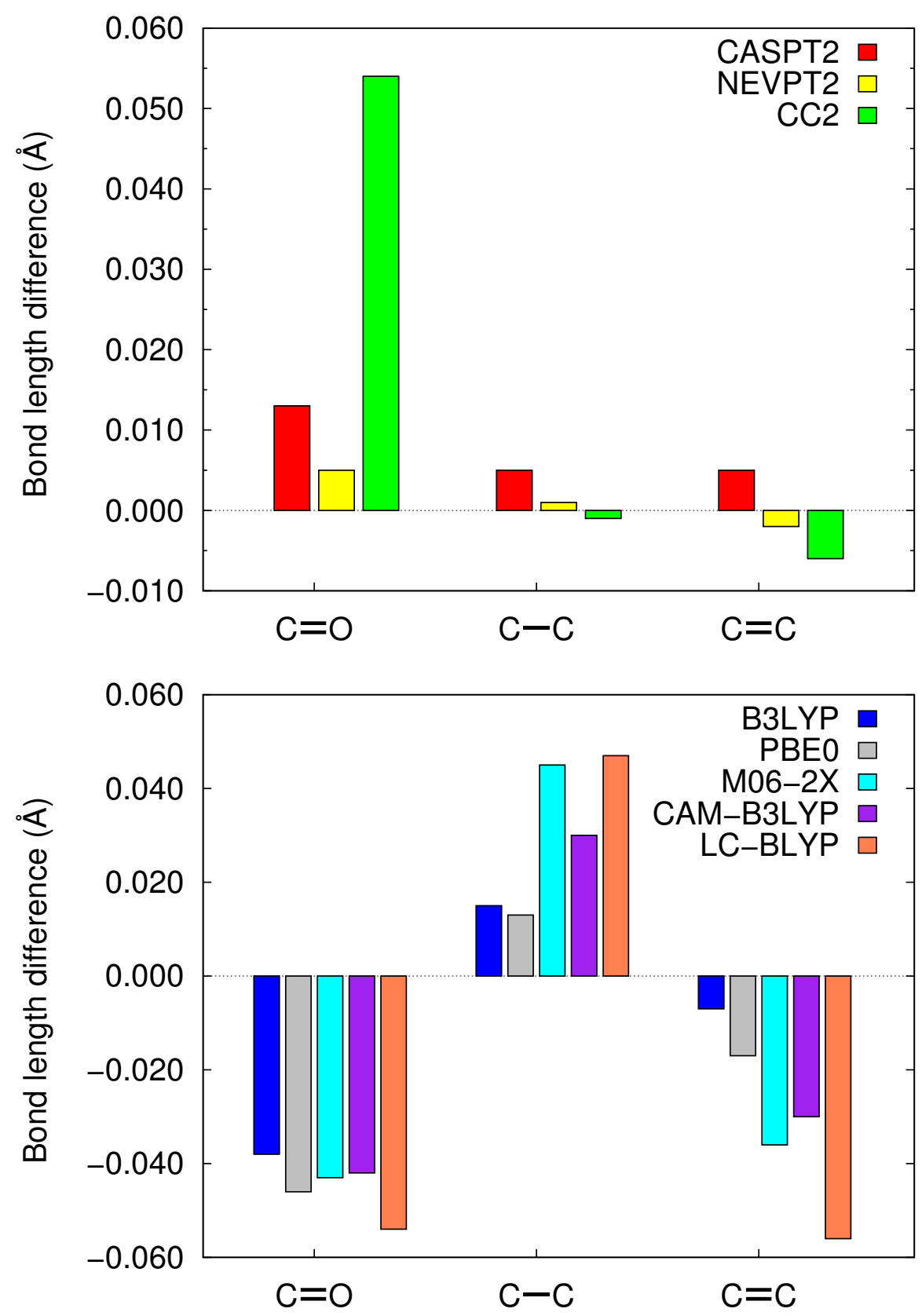

Figure 3.7: Bond-length differences $(\AA)$ between the geometries computed with different methods and the VMC reference for the $n \rightarrow \pi^{*}$ state of $s$-cis acrolein.

\section{The planar $\pi \rightarrow \pi^{*}$ excited-state geometry of $s$-trans acrolein}

We optimize the geometry of $s$-trans acrolein also in the $\pi \rightarrow \pi^{*}$ state, a case which is less documented in the literature. The $\pi \rightarrow \pi^{*}$ transition leads to an excited state of the same symmetry as the ground state if planarity $\left(C_{s}\right.$ symmetry) is imposed. For the planar optimization of this state, we limit the perturbation description to the CASPT2 method due to the limitations in code used to perform the NEVPT2 calculations as 
detailed in Section 3.3. In both the CASPT2 and VMC calculations, we employ a CAS $(4,4)$ expansion over two bonding and two anti-bonding $\pi$ orbitals, which results in the bright excited state being the third root in the state-average CASSCF calculation.

The perturbation correction yields the energetic inversion of the two highest CASSCF roots and the CASPT2 optimization following the second state leads to significantly different geometries if we use the single-state or the multi-state flavor of CASPT2. Even though the gap between the second and third state on the optimal geometry is about 0.012 Hartree, we observe a strong mixing of the eigenvectors of the second and third roots in the multi-state CASPT2 calculation, and therefore a perturbation wave function dramatically different from the zero-order reference. The problem is not ameliorated if the active space is enlarged to include more $\pi^{*}$ orbitals. Therefore, we report below the CASPT2 geometry obtained with the smallest possible CAS $(2,2)$ active space that leads to a straightforward multi-state optimization of the second state but represents a computational expedient which may be variationally rather poor.

In the VMC calculations, the use of a CAS $(4,4)$ expansion over the $\pi$ orbitals in the determinantal component of the Jastrow-Slater wave function poses instead no problems. While the relevant transition is the third state at the CASSCF level as discussed above, the introduction of the Jastrow factor reorders the two highest roots. We perform the structural relaxation in the $\pi \rightarrow \pi^{*}$ state following the second state, whose wave function is obtained in a state-average optimization at the VMC level over three states. The state of interest remains the second one in the course of the geometrical optimization and, on the optimal geometry, is energetically separated from the higher state by about 0.013 Hartree.

\begin{tabular}{lccc}
\hline & CC2 & CASPT2 & VMC \\
\hline $\mathrm{C}=\mathrm{O}$ & 1.286 & 1.279 & $1.266(2)$ \\
$\mathrm{C}-\mathrm{C}$ & 1.433 & 1.444 & $1.443(1)$ \\
$\mathrm{C}=\mathrm{C}$ & 1.436 & 1.429 & $1.428(1)$ \\
$\theta(\mathrm{C}-\mathrm{C}-\mathrm{C})$ & 125.49 & 128.39 & $123.92(6)$ \\
$\theta(\mathrm{C}-\mathrm{C}-\mathrm{O})$ & 121.30 & 119.27 & $121.64(1)$ \\
\hline
\end{tabular}

Table 3.7: CC2, CASPT2, and VMC geometries of the $\pi \rightarrow \pi^{*}\left(2^{1} \mathrm{~A}^{\prime}\right)$ state of $s$-trans acrolein in the planar geometry. The CASPT2 geometry is obtained with a CAS $(2,2)$ active space (see text).

We report our CASPT2, CC2, and VMC geometries of the planar $\pi \rightarrow \pi^{*}$ excited state in Table 3.7 and compare them in Figure 3.8. We find that the optimal CASPT2 structure obtained with the reduced CAS $(2,2)$ is in excellent agreement with the VMC geometry, the largest deviations being $0.013 \AA$ for the CO bond and about $0.001 \AA$ for the $\mathrm{CC}$ bonds. The $\mathrm{CC} 2$ structure is also in satisfactory agreement with the VMC reference, only somewhat overestimating the CO bond by $0.02 \AA$, an error which is nevertheless smaller than the corresponding one for the $n \rightarrow \pi^{*}$ state. Finally, the agreement of the SAC-CI/cc-pVDZ data from Ref. [50] with the 

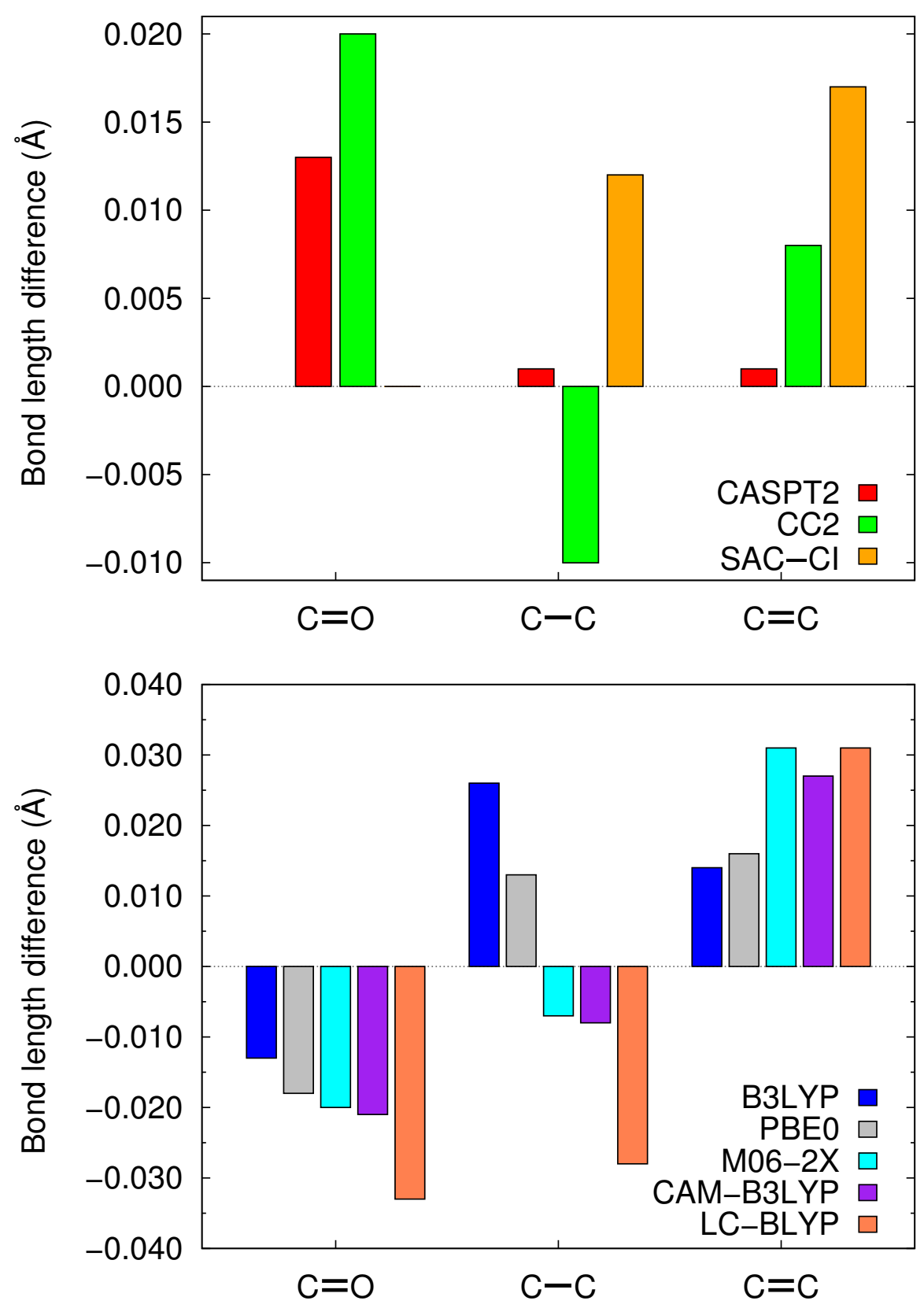

Figure 3.8: Bond-length differences $(\AA)$ between the geometries computed with different methods and the VMC reference for the $\pi \rightarrow \pi^{*}$ state of planar $s$-trans acrolein. The CASPT2 geometry is obtained with a CAS $(2,2)$ active space (see text). The SAC-CI/cc-pVDZ values are from Ref. [50].

VMC geometry is particularly good for the $\mathrm{CO}$ bond, while the $\mathrm{CC}$ bonds are slightly overestimated in SAC-CI. Finally, the TDDFT geometries are compared to VMC in Figure 3.8 (lower panel). Also in the case of the $\pi \rightarrow \pi^{*}$ excitation, there is no clear correlation between the type of functional considered (e.g. dependence on the amount of exact exchange) and the quality of the corresponding optimized geometry. 
The overall performance of TDDFT is slightly better than for the $n \rightarrow \pi^{*}$ case and the general features that emerge from the comparison are the underestimation of the CO bond, with LC-BLYP giving the largest error of $-0.033 \AA$, and the overestimation of the terminal $\mathrm{CC}$ bond.

\section{The twisted $\pi \rightarrow \pi^{*}$ excited-state geometry $s$-trans acrolein}

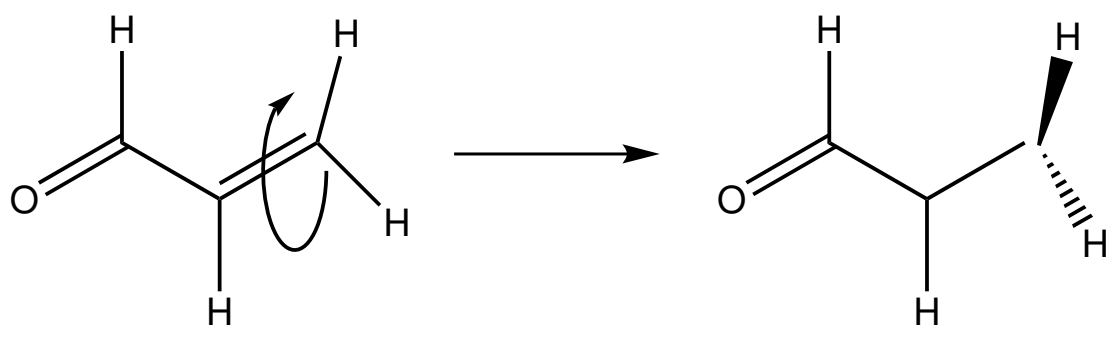

Figure 3.9: Schematic representation of the relaxation path of the $\pi \rightarrow \pi^{*}$ state of $s$-trans acrolein.

If the geometry in the $\pi \rightarrow \pi^{*}$ state of $s$-trans acrolein is optimized without imposing planarity, the minimum structure is twisted by about $90^{\circ}$ around the terminal $\mathrm{C}=\mathrm{CH}_{2}$ bond as shown in Figure 3.9. The $\pi \rightarrow \pi^{*}$ excited state of planar acrolein correlates adiabatically to a state with a strong diradical character in the twisted geometry. At the CASSCF level, the $\pi \rightarrow \pi^{*}$ wave function acquires a high weight on an up-down configuration with the HOMO orbital on the carbons in the plane and the LUMO on the terminal carbon of the twisted $\mathrm{CH}_{2}$ group. To allow a comparison with previous SAC-CI calculations [50], we keep the $\mathrm{O}-\mathrm{C}-\mathrm{C}$ frame planar and obtain a final structure with $C_{s}$ symmetry. The state with diradical character corresponds to the first root in the $\mathrm{A}^{\prime \prime}$ irreducible representation, which is also the ground state of twisted acrolein. $\mathrm{r}$ active electrons in four orbitals, namely, three $\pi$ orbitals on the carbon chain in the molecular plane and the $p$-like $\sigma$ orbital resulting from the $90^{\circ}$ rotation around the terminal $\mathrm{C}=\mathrm{CH}_{2}$ bond. We report our optimal perturbation and VMC geometries of twisted $s$-trans acrolein in the $1^{1} \mathrm{~A}^{\prime \prime}$ state in Table 3.8. The good agreement among the CASPT2, NEVPT2, and VMC structures is confirmed also for this state of acrolein. As shown in Figure 3.10, both perturbation methods only slightly overestimate the VMC bond lengths with the largest discrepancy of 0.013 $\AA$ being for the CO bond and CASPT2. On the other hand, the SAC-CI/cc-pVDZ structure from Ref. [50] only agrees with $\mathrm{VMC}$ on the $\mathrm{CO}$ bond while the $\mathrm{CC}$ bonds are overestimated with a large error of about $0.035 \AA$ for the $\mathrm{C}-\mathrm{C}$ bond and $0.02 \AA$ for the $\mathrm{C}=\mathrm{C}$ bond.

\subsubsection{Acetone}

To optimize the ground-state geometry of acetone, we impose $C_{2 v}$ symmetry and, in the $n \rightarrow \pi^{*}$ state, consider two conformations, one with the same symmetry as 


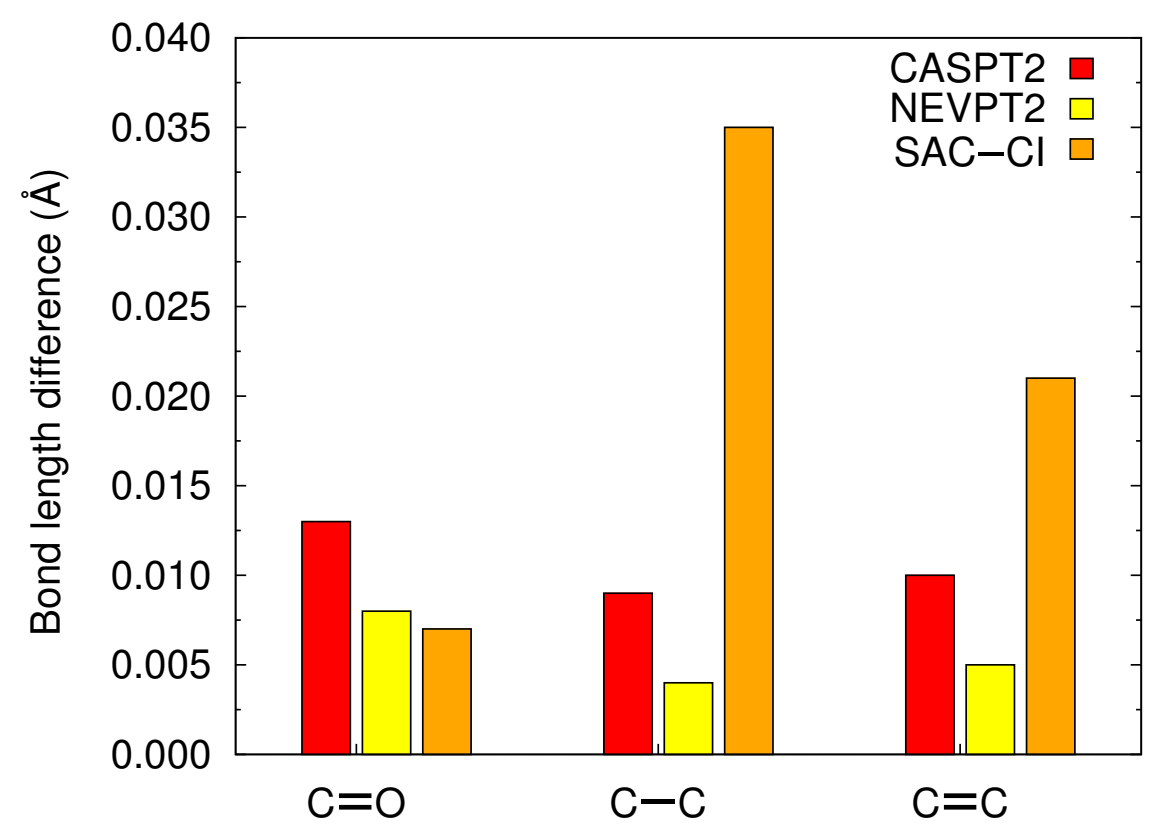

Figure 3.10: Bond-length differences $(\AA)$ between the geometries computed with different methods and the VMC reference for the $\pi \rightarrow \pi^{*}$ state of $s$-trans acrolein in the twisted geometry.

the ground state and the other one having $C_{s}$ symmetry as a consequence of the pyramidalization of the central carbon atom out of the molecular plane. In the excited state of the $C_{2 v}$ and $C_{s}$ conformations, the geometry is therefore optimized for the first root in the $\mathrm{A}_{2}$ and the $\mathrm{A}^{\prime \prime}$ irreducible representation, respectively. This second non-planar structure corresponds to a true minimum for the $n \rightarrow \pi^{*}$ state.

The ground- and excited-state geometries obtained with the correlated methods are listed in Table 3.9 and compared in Figure 3.11. As perturbation approaches, we only employ the CASPT2 method to optimize the excited-state structure due to the difficulties in the use of NEVPT2 as explained in Section 3.3. In the CASPT2 and VMC calculations, we use a CAS $(4,3)$ expansion as reference wave function, where the active space includes the lone pair on the oxygen and the bonding and antibonding $\pi$ orbitals on the $\mathrm{CO}$ bond [93]. In the ground state, the CC2, CASPT2, and VMC methods yield very close geometries, with the largest deviation of $0.017 \AA$ being between $\mathrm{CC} 2$ and VMC on the $\mathrm{CO}$ bond. For the excited states, the CASPT2 and VMC optimal structures are in very good agreement, always differing by less than $0.01 \AA$. Also for the $n \rightarrow \pi^{*}$ state of this molecule, the biggest difference is observed for the $\mathrm{CO}$ bond length computed with the $\mathrm{CC} 2$ method, which deviates from the VMC value by as much as $0.074 \AA$ when planar symmetry is imposed (see upper panel in Figure 3.11).

Similarly to the acrolein case, all TDDFT functionals tend to underestimate the $\mathrm{CO}$ bond length of acetone with respect to the optimized VMC structure, giving errors as large as $0.06 \AA$ for the LC-BLYP functional as also shown in Figure 3.11. 


\begin{tabular}{lccc}
\hline & CASPT2 & NEVPT2 & VMC \\
\hline $\mathrm{C}=\mathrm{O}$ & 1.232 & 1.226 & $1.218(0)$ \\
$\mathrm{C}-\mathrm{C}$ & 1.436 & 1.431 & $1.427(1)$ \\
$\mathrm{C}=\mathrm{C}$ & 1.454 & 1.449 & $1.444(0)$ \\
$\theta(\mathrm{C}-\mathrm{C}-\mathrm{C})$ & 123.66 & 123.97 & $123.45(5)$ \\
$\theta(\mathrm{C}-\mathrm{C}-\mathrm{O})$ & 122.82 & 122.96 & $122.80(4)$ \\
$\Theta(\mathrm{C}-\mathrm{C}-\mathrm{C}-\mathrm{H})$ & 92.14 & 92.2 & $93.14(8)$ \\
\hline
\end{tabular}

Table 3.8: CASPT2, NEVPT2, and VMC geometries of the $\pi \rightarrow \pi^{*}\left(1^{1} \mathrm{~A}^{\prime \prime}\right)$ state of $s$-trans acrolein in the twisted geometry.

\begin{tabular}{lcccc}
\hline & CC2 & CASPT2 & NEVPT2 & VMC \\
\hline & \multicolumn{5}{c}{$1^{1} \mathrm{~A}_{1}\left(C_{2 v}\right)$} \\
$\mathrm{C}=\mathrm{O}$ & 1.222 & 1.214 & 1.211 & $1.205(0)$ \\
$\mathrm{C}-\mathrm{C}$ & 1.504 & 1.509 & 1.501 & $1.502(1)$ \\
$\theta(\mathrm{C}-\mathrm{C}-\mathrm{O})$ & 121.93 & 121.91 & 121.89 & $119.80(2)$ \\
& \multicolumn{5}{c}{$1^{1} \mathrm{~A}_{2}\left(C_{2 v}\right)$} \\
$\mathrm{C}=\mathrm{O}$ & 1.422 & 1.360 & - & $1.348(1)$ \\
$\mathrm{C}-\mathrm{C}$ & 1.473 & 1.487 & - & $1.481(1)$ \\
$\theta(\mathrm{C}-\mathrm{C}-\mathrm{O})$ & 116.24 & 116.86 & - & $118.99(4)$ \\
& \multicolumn{5}{c}{$1^{1} \mathrm{~A}^{\prime \prime}\left(C_{s}\right)$} \\
$\mathrm{C}=\mathrm{O}$ & 1.404 & 1.350 & - & $1.344(1)$ \\
$\mathrm{C}-\mathrm{C}$ & 1.477 & 1.496 & - & $1.489(1)$ \\
$\theta(\mathrm{C}-\mathrm{C}-\mathrm{O})$ & 112.63 & 112.75 & - & $112.52(8)$ \\
$\Theta(\mathrm{H}-\mathrm{C}-\mathrm{C}-\mathrm{O})$ & 55.37 & 52.25 & - & $52.16(13)$ \\
\hline
\end{tabular}

Table 3.9: CC2, CASPT2, NEVPT2, and VMC bond lengths $(\AA)$ and angles (deg) of acetone in the ground state $\left(1^{1} \mathrm{~A}_{1}\right)$ and in the $n \rightarrow \pi^{*}$ excited $\left(1^{1} \mathrm{~A}_{2}\right.$ and $\left.1^{1} \mathrm{~A}^{\prime \prime}\right)$ states optimized either in $C_{2 v}$ or $C_{s}$ symmetry. We employ a cc-pVTZ basis in the CC2 and PT2 calculations, and a pVTZ' basis set in combination with a two-body Jastrow factor in VMC.

Given the good agreement between CASPT2 and VMC, the TDDFT deviations obtained here are comparable to the ones reported in an older benchmark publication [19] using CASPT2 as reference. On the other hand, the choice of CC2 as reference as in the more recent study of Ref. [25] is not recommended since the large error of $\mathrm{CC} 2$ for the $\mathrm{CO}$ bond with respect to either CASPT2 or VMC will incorrectly affect the comparison.

\subsubsection{Methylenecyclopropene}

We optimize the geometry of methylenecyclopropene in the ground and the $\pi \rightarrow \pi^{*}$ excited state maintaining planarity ( $C_{2 v}$ symmetry), so the two states have $1^{1} \mathrm{~A}_{1}$ and $1^{1} \mathrm{~B}_{2}$ symmetry, respectively. We note that the planar geometry does not correspond to a minimum for this excited state since a twisting of $90^{\circ}$ around the exo- 

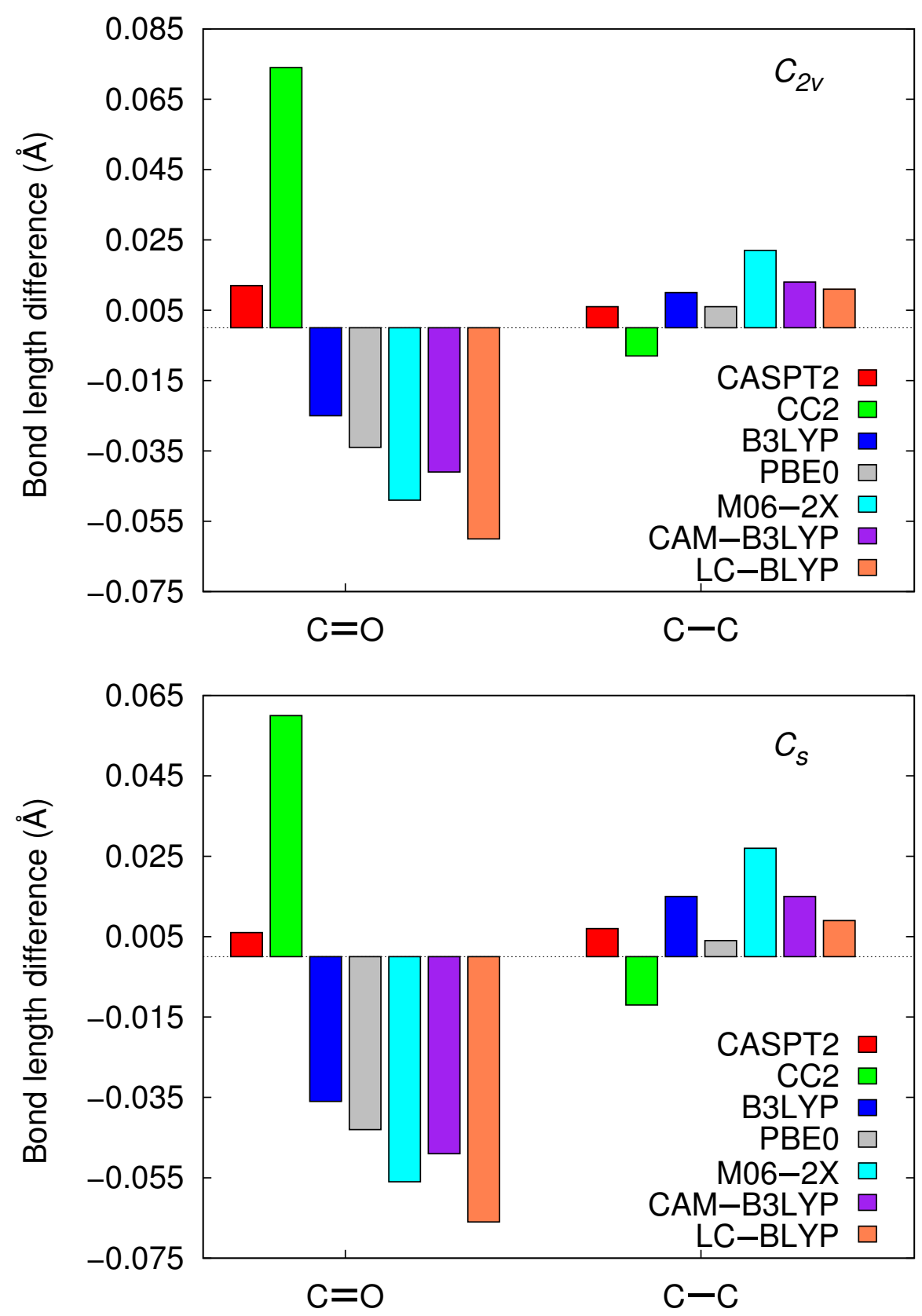

Figure 3.11: Differences in the bond lengths $(\AA)$ computed with different methods with respect to the VMC values for the $n \rightarrow \pi^{*}$ state of acetone in the $C_{2 v}$ (top) and $C_{s}$ (bottom) conformations.

cyclic $\mathrm{C}=\mathrm{CH}_{2}$ bond is expected to stabilize the state in analogy to what happens for acrolein [94]. Since we are also interested in a comparison with the CC2 and TDDFT approaches, which cannot describe diradical systems as noted above, we limit here our study to the planar $\pi \rightarrow \pi^{*}$ state of methylenecyclopropene. The atomic labels for this molecule are specified in Figure 3.12.

We present the perturbation, CC2, and VMC ground- and excited-state geome- 


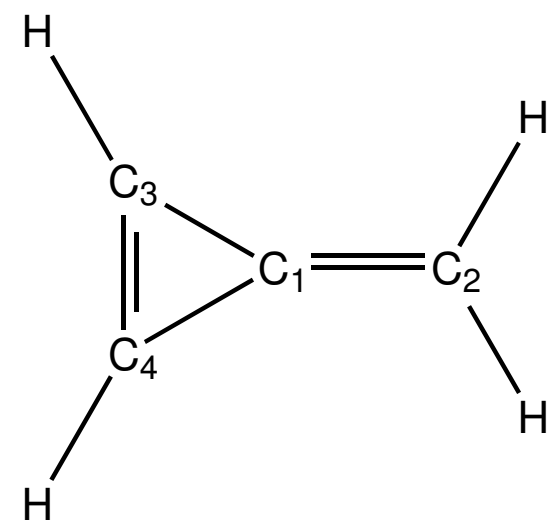

Figure 3.12: Methylenecyclopropene with atomic labels.

tries in Table 3.10. In the perturbation and VMC calculations, we construct the reference wave functions of both states as a CAS $(4,4)$ expansion over two bonding and two anti-bonding $\pi$ orbitals. We find that the ground-state perturbation and CC2 geometries are in very good agreement with the VMC structures, with all deviations being smaller than $0.01 \AA$. These ground-state structures are also compatible with the corresponding SAC-CI/cc-pVDZ geometry of Ref. [50]. In the excited state, we observe an inversion of the single and double bonds, which is well reproduced by all techniques. Also for this state, the VMC and CASPT2 structures agree very well as shown in Figure 3.13 (upper panel). The comparison with CC2 and SAC-CI reveals instead bigger errors in correspondence of the $\mathrm{C}_{3}=\mathrm{C}_{4}$ bond in the ring which both techniques overestimate by about $0.025 \AA$. The excited-state description of this part of the molecule is expected to be more critical since the excitation strongly localizes the electrons on the ring atoms [95]. On the other hand, TDDFT displays the opposite behavior, giving smaller deviations from $\mathrm{VMC}$ on this bond and non-negligible errors on $\mathrm{C}_{1}=\mathrm{C}_{2}$. Also for this molecule, the best performance is obtained with the hybrid B3LYP and PBE0 functionals (see lower panel in Figure 3.13).

\subsubsection{Propenoic acid anion}

The last molecule considered in this study is the propenoic acid anion, for which we compute the structures of the ground state and the first singlet excited state corresponding to the $n \rightarrow \pi^{*}$ excitation. The molecular structure and the relevant atomic labels are given in Figure 3.14.

We report the CC2, CASPT2, and VMC geometrical parameters of the propenoic acid anion in Table 3.11. In the ground-state optimization with the perturbation and VMC approaches, we choose a CASSCF wave function which correlates eight electrons in six orbitals, five orbitals of $\pi$ character and one $\sigma$ orbital describing the lone pair on one oxygen. At the CASPT2 level, the ground-state energy with a CAS $(8,6)$ expansion is optimal if the $\sigma$ orbital is on the $\mathrm{C}-\mathrm{O}_{1}$ bond and it is unfortunately not possible to stabilize a larger CAS also including the lone pair on the $\mathrm{C}-\mathrm{O}_{2}$ bond. The optimal ground-state geometries are characterized by almost equal $\mathrm{CO}$ bond lengths 


\begin{tabular}{lccrc}
\hline & CC2 & CASPT2 & NEVPT2 & VMC \\
\hline & \multicolumn{5}{c}{$1^{1} \mathrm{~A}_{1}$} \\
$\mathrm{C}_{1}=\mathrm{C}_{2}$ & 1.328 & 1.331 & 1.327 & $1.324(0)$ \\
$\mathrm{C}_{1}-\mathrm{C}_{3}$ & 1.438 & 1.442 & 1.434 & $1.434(0)$ \\
$\mathrm{C}_{4}=\mathrm{C}_{3}$ & 1.325 & 1.324 & 1.319 & $1.316(1)$ \\
$\theta\left(\mathrm{C}_{2}-\mathrm{C}_{1}-\mathrm{C}_{3}\right)$ & 152.56 & 152.66 & 152.62 & $152.98(4)$ \\
$\theta\left(\mathrm{C}_{1}-\mathrm{C}_{3}-\mathrm{C}_{4}\right)$ & 62.56 & 62.67 & 62.62 & $62.63(3)$ \\
& & \multicolumn{4}{c}{$1^{1} \mathrm{~B}_{2}$} \\
$\mathrm{C}_{1}=\mathrm{C}_{2}$ & 1.457 & 1.461 & - & $1.456(1)$ \\
$\mathrm{C}_{1}-\mathrm{C}_{3}$ & 1.349 & 1.360 & - & $1.351(1)$ \\
$\mathrm{C}_{4}=\mathrm{C}_{3}$ & 1.512 & 1.496 & - & $1.483(0)$ \\
$\theta\left(\mathrm{C}_{2}-\mathrm{C}_{1}-\mathrm{C}_{3}\right)$ & 145.90 & 146.64 & - & $146.66(2)$ \\
$\theta\left(\mathrm{C}_{1}-\mathrm{C}_{3}-\mathrm{C}_{4}\right)$ & 55.90 & 56.64 & - & $56.66(3)$ \\
\hline
\end{tabular}

Table 3.10: CC2, CASPT2, NEVPT2, and VMC bond lengths ( $\AA$ ) and angles (deg) of the ground $\left(1^{1} \mathrm{~A}_{1}\right)$ and the $\pi \rightarrow \pi^{*}$ excited $\left(1^{1} \mathrm{~B}_{2}\right)$ state of planar methylenecyclopropene. We employ a cc-pVTZ basis set in the CC2 and PT2 calculations, and a pVTZ' basis set in combination with a two-body Jastrow factor in VMC.

and are rather similar at all levels of theory. At the $\mathrm{CC} 2$ level, the $\mathrm{CO}$ bonds are slightly longer than the CASPT2 ones and therefore further away from the VMC values.

In the $n \rightarrow \pi^{*}$ state, one can construct two $\mathrm{CAS}(8,6)$ expansions by including the $\sigma$ orbital either on the $\mathrm{C}-\mathrm{O}_{1}$ or on the $\mathrm{C}-\mathrm{O}_{2}$ bond, as well as a $\mathrm{CAS}(10,7)$ active space comprising both lone pairs. These reference wave functions lead to very different CASPT2 and VMC geometries. If we employ the CAS $(8,6)$ expansions which give rather close CASPT2 energies on the ground-state geometry, we obtain a differential elongation of the $\mathrm{C}-\mathrm{O}_{1}$ and $\mathrm{C}-\mathrm{O}_{2}$ bonds, where the bond elongating is the one associated to the lone pair included in the active space. The geometry with a longer $\mathrm{C}-\mathrm{O}_{2}$ is consistent with the one reported in a previous CASPT2 study with the same active space [49]. Chemically, it is however unclear why the excitedstate geometry should display a differential elongation of the two $\mathrm{CO}$ bonds, which is moreover crucially dependent on the choice of the $\sigma$ orbital included in the active space. Therefore, we perform also a CASPT2 optimization with both lone pairs in a CAS $(10,7)$ expansion.

As shown in Table 3.11, the use of the CAS $(10,7)$ reference yields the expected behavior within CASPT2 and VMC: Both CO bonds are equally elongated with respect to the ground-state geometry by about 0.04-0.05 $\AA$. We note that the use of a CAS $(10,7)$ active space always leads to the same CASPT2 minimum with equal $\mathrm{CO}$ bond lengths independently of the starting geometry being the ground-state or the two CAS $(8,6)$ excited-state structures. Within VMC, the optimization with a CAS $(10,7)$ leads instead to distinct minima if we start from these different geometries. Two minima are characterized by a differential elongation of the $\mathrm{CO}$ bonds while the other one has equal $\mathrm{CO}$ bond lengths and is lower in energy by about 7 

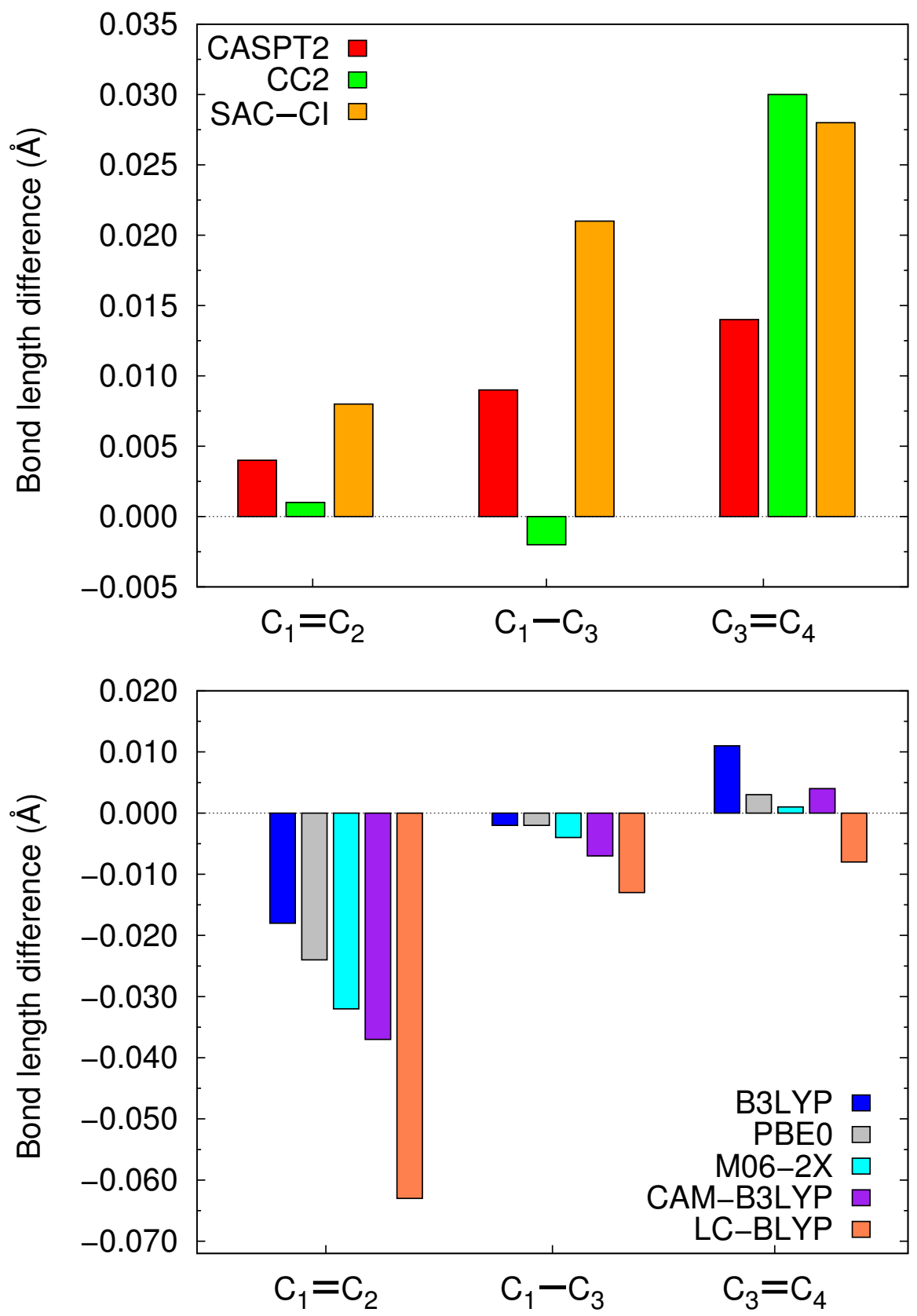

Figure 3.13: Bond-length differences $(\AA)$ between the geometries computed with different methods and the VMC reference for the $\pi \rightarrow \pi^{*}\left(1^{1} \mathrm{~B}_{2}\right)$ state of planar methylenecyclopropene.

mHartree. This optimal excited-state VMC structure is similar to the CASPT2 one but, as in the ground state, we observe differences of about $0.02 \AA$ on the $\mathrm{CO}$ bonds. In the excited state, $\mathrm{CC} 2$ yields a similar lengthening of both $\mathrm{CO}$ bonds as observed at the CASPT2 and VMC level (See Figure 3.15). The biggest difference with the other correlated approaches is the excessive lengthening of the $\mathrm{C}-\mathrm{O}_{2}$ bond, which is 0.03 and $0.05 \AA$ longer than the CASPT2 and VMC values, respectively. Finally, 


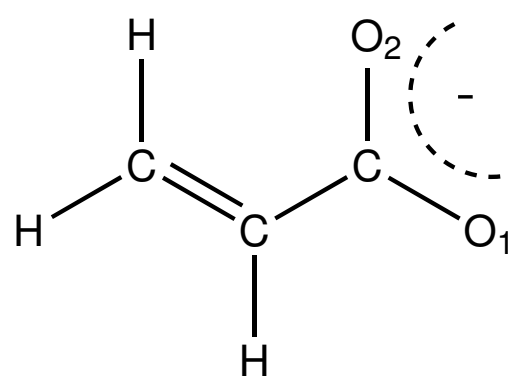

Figure 3.14: Propenoic acid anion with atomic labels.

\begin{tabular}{lccc}
\hline & CC2 & CASPT2 & VMC \\
\hline & & $1^{1} \mathrm{~A}^{\prime}$ \\
$\mathrm{C}-\mathrm{O}_{1}$ & 1.265 & 1.260 & $1.245(1)$ \\
$\mathrm{C}-\mathrm{O}_{2}$ & 1.261 & 1.253 & $1.241(0)$ \\
$\mathrm{C}-\mathrm{C}$ & 1.521 & 1.531 & $1.526(1)$ \\
$\mathrm{C}=\mathrm{C}$ & 1.335 & 1.338 & $1.324(0)$ \\
$\theta\left(\mathrm{O}_{1}-\mathrm{C}-\mathrm{C}\right)$ & 114.02 & 114.00 & $113.95(5)$ \\
$\theta(\mathrm{C}-\mathrm{C}-\mathrm{C})$ & 123.48 & 123.68 & $124.03(6)$ \\
$\theta\left(\mathrm{O}_{1}-\mathrm{C}-\mathrm{O}_{2}\right)$ & 129.59 & 129.69 & $129.72(4)$ \\
& & $1^{1} \mathrm{~A}^{\prime \prime}$ & \\
$\mathrm{C}-\mathrm{O}_{1}$ & 1.311 & 1.309 & $1.291(1)$ \\
$\mathrm{C}-\mathrm{O}_{2}$ & 1.331 & 1.299 & $1.284(1)$ \\
$\mathrm{C}-\mathrm{C}$ & 1.385 & 1.387 & $1.379(0)$ \\
$\mathrm{C}=\mathrm{C}$ & 1.415 & 1.412 & $1.401(0)$ \\
$\theta\left(\mathrm{O}_{1}-\mathrm{C}-\mathrm{C}\right)$ & 130.41 & 128.39 & $128.33(7)$ \\
$\theta(\mathrm{C}-\mathrm{C}-\mathrm{C})$ & 123.43 & 124.70 & $124.49(7)$ \\
$\theta\left(\mathrm{O}_{1}-\mathrm{C}-\mathrm{O}_{2}\right)$ & 102.32 & 103.98 & $107.18(7)$ \\
\hline
\end{tabular}

Table 3.11: CC2, CASPT2, NEVPT2, and VMC bond lengths ( $\left({ }^{\prime}\right)$ and angles (deg) of the ground and $n \rightarrow \pi^{*}$ excited states of the propenoic acid anion. We employ a cc-pVTZ basis set in the CC2 and CASPT2 calculations, and a pVTZ' basis set in combination with a two-body Jastrow factor in VMC.

the geometries optimized at the TDDFT level share the common feature of having $\mathrm{CO}$ bonds of equal length which are very close to the VMC values, while bigger differences are observed for the $\mathrm{C}-\mathrm{C}$ bond. 


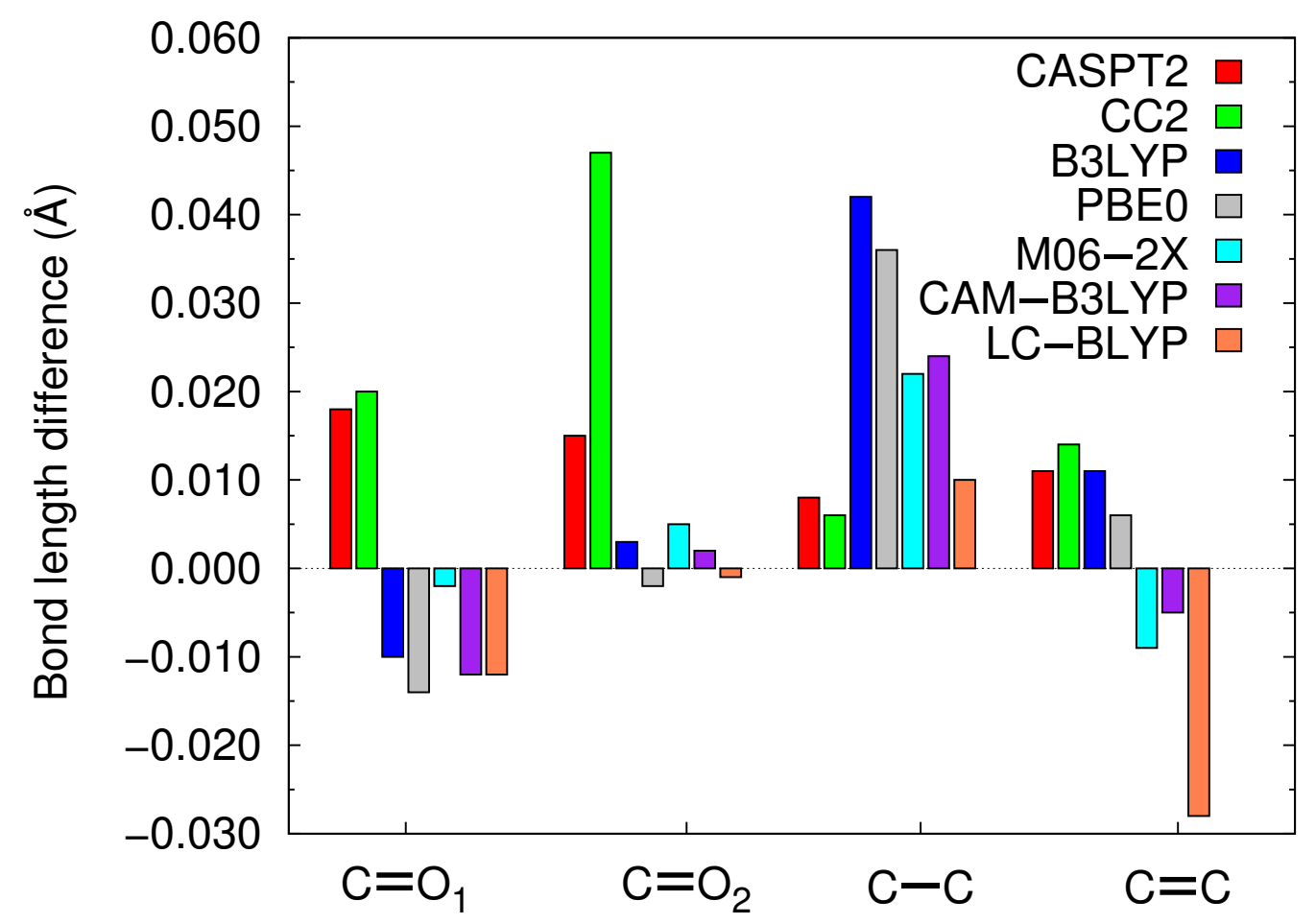

Figure 3.15: Bond-length differences $(\AA)$ between the geometries computed with different methods and the VMC reference for the $n \rightarrow \pi^{*}$ state of propenoic acid anion. 


\subsection{Conclusions}

In this paper, we have presented a thorough investigation of the performance of a variety of first-principle approaches in optimizing the geometries of a set of small organic chromophores, with particular focus on their excited-state structures. The primary goal was to assess the accuracy of the VMC geometries through a detailed comparison with those obtained with other highly-correlated approaches such as multireference perturbation approaches (CASPT2 and NEVPT2), coupled cluster (CC2) and SAC-CI, which have been used in previous benchmark studies of TDDFT or other correlated approaches.

For this aim, we revisited the perturbation calculations for these molecules with special emphasis on the construction of the reference wave function since the choice of active space may significantly affect the excited-state optimal geometry as in the case of the propenoic acid anion.

As shown in Figure 3.16, the level of agreement between the different methods and VMC can be discussed in terms of the maximum absolute deviations (MXAD) with respect to the VMC excited-state bond lengths. For the perturbation theories and $\mathrm{CC} 2$, the MXAD occurs for the $\mathrm{CO}$ bond of the carbonyl compounds and for the double CC bond in the ring of MCP, while we find that SAC-CI consistently gives a worse description of the $\mathrm{CC}$ bond lengths. We observe that the $\mathrm{VMC}$ and perturbation structures are always very close: The MXADs are extremely small at the NEVPT2 level and consistently around $0.013 \AA$ within CASPT2, with the only exception of the more problematic case of the propenoic acid anion with its bigger error of about $0.018 \AA$. With maximum absolute deviations of $0.04-0.075 \AA$, the comparison with CC2 confirms the lack of accuracy of this method in the description of the CO bond in the $n \rightarrow \pi^{*}$ excited states as already reported in Ref. [26]. CC2 is instead able to provide a somewhat better description of the $\pi \rightarrow \pi^{*}$ excited states of acrolein and MCP, where the MXADs are about 0.02-0.03 $\AA$. The maximum errors on the SAC-CI geometries are of the same order of magnitude as those on the CC2 structures.

At the TDDFT level, most functionals give the largest errors on the CO bond of the carbonyl compounds in the $n \rightarrow \pi^{*}$ excited states, with the exception of the propenoic acid anion where all functionals fail on the CC bonds. As regards the $\pi \rightarrow$ $\pi^{*}$ excitations, the MXAD for the different functionals is always on the exocyclic double bond of MCP while it occurs on different bonds of acrolein. Manifestly, it is not possible to identify a clear correlation between the defining features of the different functional investigated (e.g. amount of exact exchange) and the accuracy of the geometry obtained. Furthermore, while the poorest results are obtained with the LC-BLYP functional, it is not straightforward to pinpoint a single functional as the one offering the best overall performance in the optimization of the excited-state structures of these molecules.

In conclusion, our study demonstrates that QMC (also in its simplest VMC variant) can be successfully employed as a robust method for the accurate optimization of ground- and excited-state structures also in cases where other correlated or TDDFT methods fail. Thanks to its favorable computational scaling with the system 

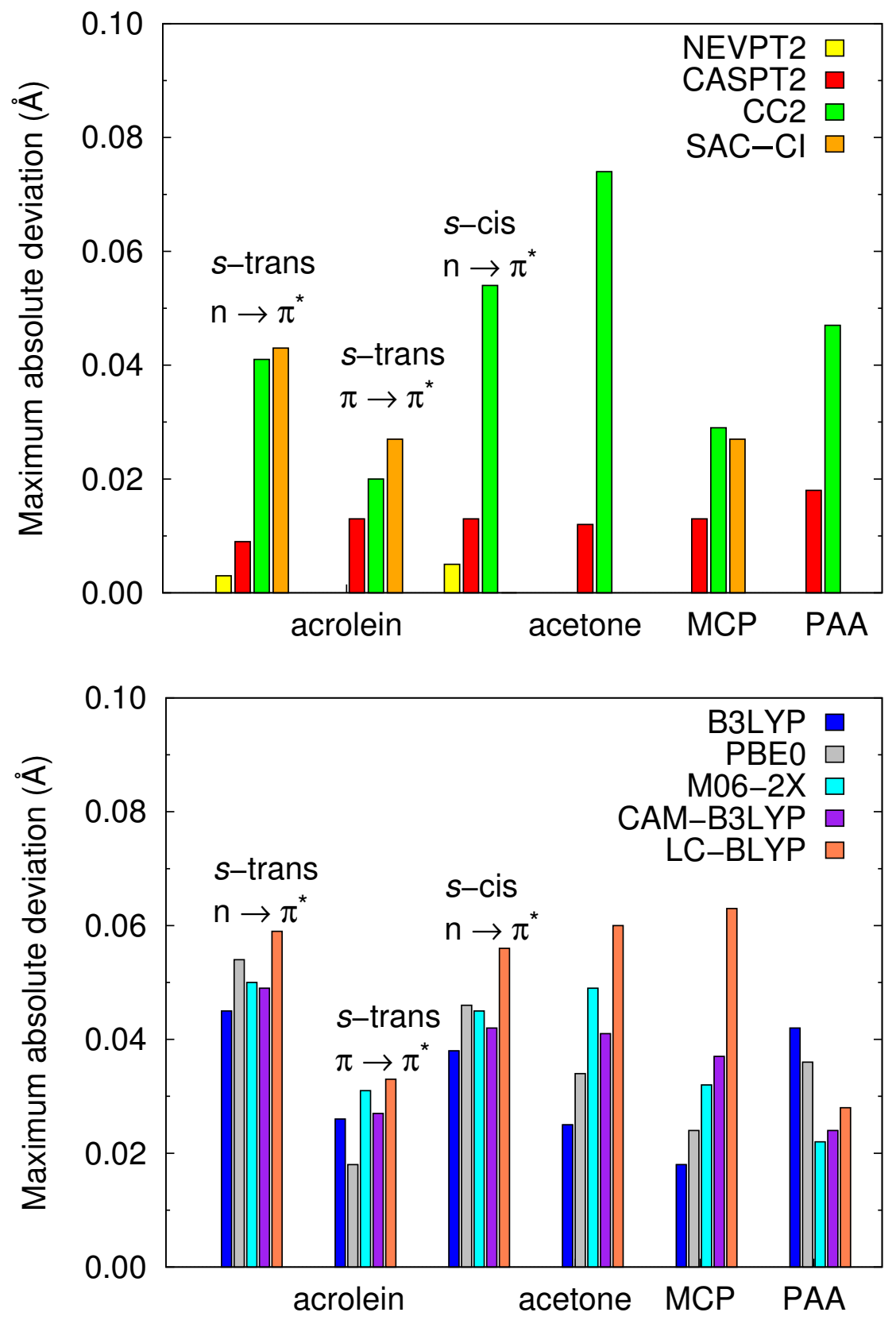

Figure 3.16: Maximum absolute deviations $(\AA)$ on the bond lengths in the excited state with respect to the VMC optimal geometries.

size, QMC represents therefore a promising alternative to other methods ordinarily employed in quantum chemistry for the structural study also of more complex molecules. 


\section{Bibliography}

[1] M. Casida, J. Mol. Struct. (Theochem) 914, 3 (2009).

[2] M. E. Casida and M. Huix-Rotllant, Annu. Rev. Phys. Chem. 63, 287 (2012).

[3] M. Marques, T. N. Maitra, F. M. S. Nogueira, E. Gross, and A. Rubio, Fundamentals of Time-Dependent Density Functional Theory (Springer, Heidelberg, 2012), pp. 53-99.

[4] A. Dreuw, J. L. Weisman, and M. Head-Gordon, J. Chem. Phys. 119, 2943 (2003).

[5] M. E. Casida, J. Chem. Phys. 122, 054111 (2005).

[6] T. Yanai, D. P. Tew, and N. C. Handy, Chem. Phys. Lett. 393, 51 (2004).

[7] H. Iikura, T. Tsuneda, T. Yanai, and K. Hirao, J. Chem. Phys. 115, 3540 (2001).

[8] Y. Tawada, T. Tsuneda, S. Yanagisawa, T. Yanai, and K. Hirao, J. Chem. Phys. 120, 8425 (2004).

[9] M. Chiba, T. Tsuneda, and K. Hirao, J. Chem. Phys. 124, 144106 (2006).

[10] O. A. Vydrov, J. Heyd, A. V. Krukau, and G. E. Scuseria, J. Chem. Phys. 125, 074106 (2006).

[11] O. A. Vydrov and G. E. Scuseria, J. Chem. Phys. 125, 234109 (2006).

[12] J.-D. Chai and M. Head-Gordon, J. Chem. Phys. 128, 084106 (2008).

[13] J.-D. Chai and M. Head-Gordon, Phys. Chem. Chem. Phys. 10, 6615 (2008).

[14] F. Furche and R. Ahlrichs, J. Chem. Phys. 117, 7433 (2002).

[15] G. Scalmani, J. M. Frisch, B. Mennucci, J. Tomasi, R. Cammi, and V. Barone, J. Chem. Phys. 124, 094107 (2006).

[16] M. A. Rohrdanz and M. J. Herbert, J. Chem. Phys. 129, 034107 (2008).

[17] D. Jacquemin, E. A. Perpète, G. E. Scuseria, I. Ciofini, and C. Adamo, Chem. Phys. Lett. 465, 226 (2008). 
[18] D. Jacquemin, V. Wathelet, E. A. Perpète, and C. Adamo, J. Chem. Theory Comput. 5, 2420 (2009).

[19] C. A. Guido, D. Jacquemin, C. Adamo, and B. Mennucci, J. Phys. Chem. A 114, 13402 (2010).

[20] R. Send, M. Kühn, and F. Furche, J. Chem. Theory Comput. 7, 2376 (2011).

[21] S. S. Leang, F. Zahariev, and M. S. Gordon, J. Chem. Phys. 136, 104101 (2012).

[22] D. Jacquemin, A. Planchat, C. Adamo, and B. Mennucci, J. Chem. Theory Comput. 8, 2359 (2012).

[23] D. Bousquet, R. Fukuda, P. Maitarad, D. Jacquemin, A. Ciofini, C. Adamo, and M. Ehara, J. Chem. Theory Comput. 9, 2368 (2013).

[24] A. D. Laurent and D. Jacquemin, Int. J. Quantum Chem. 113, 2019 (2013).

[25] C. A. Guido, S. Knecht, J. Kongsted, and B. Mennucci, J. Chem. Theory Comput. 9, 2209 (2013).

[26] A. Köhn and C. Hätting, J. Chem. Phys. 119, 5021 (2003).

[27] W. M. C. Foulkes, L. Mitas, R. J. Needs, and G. Rajagopal, Rev. Mod. Phys. 73, 33 (2001).

[28] J. Kolorenc and L. Mitas, Rep. Prog. Phys. 74, 026502 (2011).

[29] B. M. Austin, D. Y. Zubarev, and W. A. Lester, Chem. Rev. 112, 263 (2012).

[30] F. Schautz and C. Filippi, J. Chem. Phys. 120, 10931 (2004).

[31] F. Schautz, F. Buda, and C. Filippi, J. Chem. Phys. 121, 5836 (2004).

[32] N. Drummond, A. Williamson, R. Needs, and G. Galli, Phys. Rev. Lett. 95, 096801 (2005).

[33] F. Cordova, L. J. Doriol, A. Ipatov, M. E. Casida, C. Filippi, and A. Vela, J. Chem. Phys. 127, 164111 (2007).

[34] E. Tapavicza, I. Tavernelli, U. Rothlisberger, C. Filippi, and M. E. Casida, J. Chem. Phys. 129, 124108 (2008).

[35] P. M. Zimmerman, J. Toulouse, Z. Zhang, C. B. Musgrave, and C. J. Umrigar, J. Chem. Phys. 131, 124103 (2009).

[36] C. Filippi, M. Zaccheddu, and F. Buda, J. Chem. Theory Comput. 5, 2074 (2009).

[37] O. Valsson and C. Filippi, J. Chem. Theory Comput. 6, 1275 (2010). 
[38] R. Send, O. Valsson, and C. Filippi, J. Chem. Theory Comput. 7, 444 (2011).

[39] C. Filippi, F. Buda, L. Guidoni, and A. Sinicropi, J. Chem. Theory Comput. 8, 112 (2012).

[40] O. Valsson, C. Angeli, and C. Filippi, Phys. Chem. Chem. Phys. 14, 11015 (2012).

[41] O. Valsson, P. Campomanes, I. Tavernelli, U. Rothlisberger, and C. Filippi, J. Chem. Theory Comput. 9, 2441 (2013).

[42] G. Ghigo, B. O. Roos, and P.-Å. Malmqvist, Chem. Phys. Lett. 396, 142 (2004).

[43] C. Angeli, R. Cimiraglia, S. Evengelisti, T. Leininger, and J.-P. Malrieu, J. Chem. Phys. 114, 10252 (2001).

[44] C. Attaccalite and S. Sorella, Phys. Rev. Lett. 100, 114501 (2008).

[45] S. Sorella and L. Capriotti, J. Chem. Phys. 133, 234111 (2010).

[46] M. Barborini and L. Guidoni, J. Chem. Phys. 137, 224309 (2012).

[47] S. Saccani, C. Filippi, and S. Moroni, J. Chem. Phys. 138, 084109 (2013).

[48] C. J. Umrigar, J. Toulouse, C. Filippi, S. Sorella, and R. G. Hennig, Phys. Rev. Lett. 98, 110201 (2007).

[49] S. C. Page and M. Olivucci, J. Comput. Chem. 24, 298 (2003).

[50] R. Cammi, R. Fukuda, M. Ehara, and H. Nakatsuij, J. Chem. Phys. 133, 024104 (2010).

[51] C. Filippi and C. J. Umrigar, J. Chem. Phys. 105, 213 (1996), as Jastrow correlation factor, we use the exponential of the sum of three fifth-order polynomials of the electron-nuclear (e-n), the electron-electron (e-e), and of pure 3-body mixed e-e and e-n distances, respectively. The Jastrow factor is adapted to deal with pseudo-atoms, and the scaling factor $\kappa$ is set to 0.6 a.u. The 2-body Jastrow factor includes five parameters in the e-e terms and four parameters for each atom type in the e-n terms. The 3-body Jastrow factor has 15 additional parameters per atom type. In both Jastrow factors, the same scaling parameter $\kappa$ is used for all inter-particle distances. For $s$-trans acrolein ( 3 atom types), we have therefore a total of 17 and 62 parameters in the 2-body and the 3-body Jastrow factor, respectively.

[52] S. Moroni, S. Saccani, and C. Filippi, J. Chem. Theory Comput. 10, 4823 (2014).

[53] C. Filippi, R. Assaraf, and S. Moroni, J. Chem. Phys. 144, 194105 (2016).

[54] C. Filippi and C. J. Umrigar, Phys. Rev. B 61, R16291 (2000). 
[55] Gaussian 09 Revision A.02, M. J. Frisch, G. W. Trucks, H. B. Schlegel, G. E. Scuseria, M. A. Robb, J. R. Cheeseman, G. Scalmani, V. Barone, B. Mennucci, G. A. Petersson, H. Nakatsuji, M. Caricato, X. Li, H. P. Hratchian, A. F. Izmaylov, J. Bloino, G. Zheng, J. L. Sonnenberg, M. Hada, M. Ehara, K. Toyota, R. Fukuda, J. Hasegawa, M. Ishida, T. Nakajima, Y. Honda, O. Kitao, H. Nakai, T. Vreven, J. A. Montgomery, Jr., J. E. Peralta, F. Ogliaro, M. Bearpark, J. J. Heyd, E. Brothers, K. N. Kudin, V. N. Staroverov, R. Kobayashi, J. Normand, K. Raghavachari, A. Rendell, J. C. Burant, S. S. Iyengar, J. Tomasi, M. Cossi, N. Rega, J. M. Millam, M. Klene, J. E. Knox, J. B. Cross, V. Bakken, C. Adamo, J. Jaramillo, R. Gomperts, R. E. Stratmann, O. Yazyev, A. J. Austin, R. Cammi, C. Pomelli, J. W. Ochterski, R. L. Martin, K. Morokuma, V. G. Zakrzewski, G. A. Voth, P. Salvador, J. J. Dannenberg, S. Dapprich, A. D. Daniels, Ö. Farkas and J. B. Foresman, J. V. Ortiz, J. Cioslowski, and D. J. Fox, Gaussian Inc. Wallingford CT 2009.

[56] A. D. Becke, Phys. Rev. A 38, 3098 (1988).

[57] C. Lee, W. Yang, and R. G. Parr, Phys. Rev. B 37, 785 (1988).

[58] A. D. Becke, J. Chem. Phys. 98, 5648 (1993).

[59] P. J. Stephens, F. J. Devlin, C. F. Chabalowski, and M. J. Frisch, J. Phys. Chem. 98, 11623 (1994).

[60] J. P. Perdew, K. Burke, and M. Ernzerhof, J. Chem. Phys. 105, 9982 (1996).

[61] C. Adamo and V. Barone, J. Chem. Phys. 110, 6158 (1999).

[62] M. Ernzerhof and G. E. Scuseria, J. Chem. Phys. 110, 5029 (1999).

[63] Y. Zhao and D. Truhlar, Theor. Chem. Acc. 120, 215 (2008).

[64] O. Christiansen, H. Koch, and P. Jørgensen, Chem. Phys. Lett. 243, 409 (1995).

[65] C. Hättig and F. Weigend, J. Chem. Phys. 113, 5154 (2000).

[66] C. Hättig and A. Köhn, J. Chem. Phys. 117, 6939 (2002).

[67] C. Hättig, Adv. in Quantum Chem. 50, 37 (2005).

[68] CFOUR, Coupled-Cluster techniques for Computational Chemistry, a quantum-chemical program package by J.F. Stanton, J. Gauss, M.E. Harding, P.G. Szalay with contributions from A.A. Auer, R.J. Bartlett, U. Benedikt, C. Berger, D.E. Bernholdt, Y.J. Bomble, L. Cheng, O. Christiansen, M. Heckert, O. Heun, C. Huber, T.-C. Jagau, D. Jonsson, J. Jusélius, K. Klein, W.J. Lauderdale, D.A. Matthews, T. Metzroth, L.A. Mück, D.P. O’Neill, D.R. Price, E. Prochnow, C. Puzzarini, K. Ruud, F. Schiffmann, W. Schwalbach, S. Stopkowicz, A. Tajti, J. Vázquez, F. Wang, J.D. Watts and the integral packages MOLECULE (J. Almlöf and P.R. Taylor), PROPS (P.R. Taylor), ABACUS (T. 
Helgaker, H.J. Aa. Jensen, P. Jørgensen, and J. Olsen), and ECP routines by A. V. Mitin and C. van Wüllen. For the current version, see http://www.cfour.de. (accessed July 12, 2012).

[69] G. Karlström, R. Lindh, P.-Å. Malmqvist, B. O. Roos, U. Ryde, V. Veryazov, P.O. Widmark, M. Cossi, B. Schimmelpfennig, P. Neogrady, and L. Seijo, Comput. Mater. Sci. 28, 222 (2003).

[70] K. Andersson, P.-A. Malmqvist, B. O. Roos, A. J. Sadlej, and K. Wolinski, J. Phys. Chem. 94, 5483 (1990).

[71] K. Andersson, P.-A. Malmqvist, and B. O. Roos, J. Chem. Phys. 96, 1218 (1992).

[72] C. Angeli, R. Cimiraglia, and J.-P. Malrieu, Chem. Phys. Lett. 350, 297 (2001).

[73] C. Angeli, R. Cimiraglia, and J.-P. Malrieu, J. Chem. Phys. 117, 9138 (2002).

[74] F. Neese, Comput. Mol. Sci. 2, 73 (2012).

[75] CHAMP is a quantum Monte Carlo program package written by C. J. Umrigar, C. Filippi and collaborators.

[76] M. Burkatzki, C. Filippi, and M. Dolg, J. Chem. Phys. 126, 234105 (2007).

[77] For the hydrogen atom, we use a more accurate BFD pseudopotential and basis set. Dolg, M.; Filippi, C., private communication.

[78] M. W. Schmidt, K. K. Baldridge, J. A. Boatz, S. T. Elbert, M. S. Gordon, J. H. Jensen, S. Koseki, N. Matsunaga, K. A. Nguyen, S. Su, T. L. Windus, M. Dupuis, and J. A. M. Jr, J. Comput. Chem. 14, 1347 (1993).

[79] T. H. Dunning Jr, J. Chem. Phys. 90, 1007 (1989).

[80] K. A. Peterson, D. E. Woon, and T. H. Dunning Jr, J. Chem. Phys. 100, 7410 (1994).

[81] A. Wilson, T. van Mourik, and T. H. Dunning Jr, J. Mol. Struct. (Theochem) 388, 339 (1997).

[82] F. Weigend, Phys. Chem. Chem. Phys. 8, 1057 (2006).

[83] We take the diffuse functions for the heavy atoms atoms from the aug-cc-pVXZ basis sets in the EMSL Basis Set Library (http://bse.pnl.gov).

[84] F. Aquilante, V. Barone, and B. Roos, J. Chem. Phys. 119, 12323 (2003).

[85] I. Gadaczeck, K. Krause, K. J. Hintze, and T. Bredow, J. Chem. Theory Comput. 8, 986 (2012). 
[86] C. Angeli, S. Borini, L. Ferrighi, and R. Cimiraglia, J. Chem. Phys. 122, 114304 (2005).

[87] C. Angeli, S. Borini, L. Ferrighi, and R. Cimiraglia, J. Mol. Struct. (Theochem) 718, 55 (2005).

[88] B. Saha, M. Ehara, and H. Nakatsuji, J. Chem. Phys. 125, 014316 (2006).

[89] M. Reguero, M. Olivucci, F. Bernardi, and M. A. Robb, J. Am. Chem. Soc. 116, 2103 (1994).

[90] A. M. Losa, I. F. Galván, M. A. Aguilar, and M. E. Martín, J. Phys. Chem. B 111, 9864 (2007).

[91] S. R. Gwaltney and R. J. Bartlett, J. Chem. Phys. 110, 62 (1999).

[92] In MOLCAS, we can only perform CASSCF ground-state calculations of $s$ trans acrolein with a $\mathrm{CAS}(6,5)$ expansion containing the lone pair parallel to the $\mathrm{CO}$ bond. We cannot stabilize the expansions including the other lone pair or both lone pairs. The CASSCF energy of the $n \rightarrow \pi^{*}$ excited $\left(1^{1} \mathrm{~A}^{\prime \prime}\right)$ state is instead lower if the $\sigma$ orbital corresponds to the lone pair orthogonal to the $\mathrm{CO}$ group. To describe the two states with the same active space and stabilize the same CAS on the ground state, we run a SA calculation without symmetry on both states and with a ratio of $995 / 5$ in the weights for the two roots. The CASPT2 geometry optimization relaxing the first root in this fictitious SACASSCF calculation converges to the same geometry calculated in a singlestate run with the lone pair parallel to the $\mathrm{CO}$ bond in the active space, regardless the choice of the basis set.

[93] We note that, for both excited states, a CAS(4,3) zero-order wave function is equivalent to a $\operatorname{CAS}(2,2)$ expansion only including the oxygen lone pair and the $\pi^{*}$ orbital, according to the Brillouin's theorem.

[94] R. P. Johnson and M. W. Schmidt, J. Am. Chem. Soc. 103, 3244 (1981).

[95] M. Merchán, R. González-Luque, and B. O. Roos, Theor. Chem. Acc. 94, 143 (1996). 


\section{Chapter 4}

\section{Solvent effects on excited-state structures: A quantum Monte Carlo and density functional study ${ }^{\dagger}$}

We present the first application of quantum Monte Carlo (QMC) in its variational flavor combined with the polarizable continuum model (PCM) to perform excited-state geometry optimization in solution. Our implementation of the PCM model is based on a reaction field which includes both volume and surface polarization charges, and is determined self-consistently with the molecular wave function during the QMC optimization of the solute geometry. For acrolein, acetone, methylenecyclopropene, and the propenoic acid anion, we compute the optimal exited-state geometries in water and compare our results with the structures obtained with second-order perturbation theory (CASPT2) and other correlated methods, and with time-dependent density functional theory (TDDFT). We find that QMC predicts a structural response to solvation in good agreement with CASPT2 with the only exception of the $\pi \rightarrow \pi^{*}$ state of acrolein where the robustness of the QMC geometry must be contrasted to the sensitivity of the perturbation result to the details of the calculation. As regards TDDFT, we show that all investigated functionals systematically overestimate the geometrical changes from the gas phase to solution, sometimes giving bond variations opposite in trend to QMC.

\subsection{Introduction}

A realistic theoretical description of photo-excitations in chemical systems must take into account that these processes generally occur in a condensed medium such as a liquid solution, which may significantly affect the molecular properties of the active

$\dagger$ This chapter has been published as R. Guareschi, F. M. Floris, C. Amovilli, and C. Filippi, "Solvent effects on excited-state structures: A quantum Monte Carlo and density functional study", $J$. Chem. Theory Comput. 2014, 10, 5528-5537 
species with respect to the gas phase. The most common approach to include solvation effects is to describe the solute at the quantum mechanical level and the solvent classically. In this framework, the solvent can be represented at different levels of sophistication, preserving its explicit atomistic structure or, at the other extreme, as a continuum medium [1-7].

The methods based on a continuum solvent offer a cost effective manner to approximately include an average description of the environment and account for important polarization contributions in processes such as absorption, emission, and structural relaxation in solution. Among the numerous variants of continuum approaches, the most popular nowadays is the polarizable continuum model (PCM) [1, 6], where the solvent is a dielectric medium surrounding a molecule enclosed in a cavity and the solute-solvent interactions are mimicked via polarization charges distributed on the surface of the cavity and sometimes also in the bulk of the continuum medium (volume polarization charges) $[1,8]$.

As regards the treatment of the solute and its structural response in the excited state, a plethora of quantum chemical methods is available whose performance has been primarily investigated in the gas phase. The ability of an approach to treat an isolated species does however not ensure an equally good quality of its excitedstate response to the presence of a solvent. In benchmarking time-dependent density functional theory (TDDFT) [9-11], the focus has in fact recently moved to establish its performance in describing optimal excited-state structures, fluorescence emission, and adiabatic absorption beyond the gas phase. For this purpose, the combination of TDDFT with PCM techniques appears to be the simplest and most effective mean to obtain a fast, qualitatively correct analysis of molecular properties in a solvated environment and has in fact been employed to this purpose in different flavors [1219], the most recent one in a structural investigation also including the response of the exact TDDFT excited-state density [20]. These studies suffered however for the lack of good reference data, which can in principle be provided by accurate wave function methods.

In this context, quantum Monte Carlo (QMC) represents an accurate and robust highly-correlated approach to investigate the properties of a solvated molecular system. QMC has been shown [21-26] to yield excitation energies of a variety of chromophores in good agreement with multi-reference second-order perturbation theories like CASPT2 in its most recent formulation [27] and the $n$-electron valence perturbation theory (NEVPT2) [28], while offering a more favorable scaling for large systems ( $N^{4}$ with $N$ the number of electrons) than either perturbation approach. Ground- and excited-state geometry optimizations [22, 29-32] can be carried out within QMC in its variational (VMC) flavor thanks to a robust wave function optimization procedure in state-specific [33] and state-average [21] fashion, and an efficient implementation of the computation of interatomic forces with a finite-variance estimator [29,30]. Recently, our group has presented a detailed study of the groundand excited-state geometry optimization of a set of representative organic molecules in the gas phase [34], obtaining QMC optimal structures consistent with those resulting from perturbation theory and providing robust reference data whenever the application of a perturbation approach proved problematic. 
The aim of this work is to provide an accurate PCM benchmark of both groundand excited-state structures of prototypical organic solutes, which we treat here at the QMC level. For this purpose, we combine for the first time structural optimization in VMC with our QMC/PCM approach [35-38] to include a continuum solvent determined self-consistently with the solute wave function in terms of surface and volume polarization charges. For a set of small organic chromophores representative of $n \rightarrow \pi^{*}$ and $\pi \rightarrow \pi^{*}$ transitions, we compute the optimal excited-state geometries in water within VMC and compare them with those resulting from TDDFT and CASPT2 in the same medium. We find that QMC yields structural changes due to the presence of the solvent in good agreement with the predictions of the CASPT2 method. Furthermore, it provides a robust estimate of the geometry of the $\pi \rightarrow \pi^{*}$ state of acrolein, where the perturbation results are instead strongly dependent on the computational ingredients entering the calculation. Finally, all TDDFT functionals investigated here tend to overestimate the geometrical response to the solvent in the excited states and yield an even larger error with respect to the correlated approaches than in the gas phase.

The paper is organized as follows. In Section 4.2, we discuss various methodological aspects related to the computation of interatomic gradients in QMC/PCM and, in Section 4.3, we present the computational details. In Section 4.4, we present a detailed analysis of our results and conclude in Sec. 4.5.

\subsection{Methods}

We focus here on the excited-state geometry optimization of small organic molecules and include solvation effects within the polarizable continuum model (PCM) $[1,6$, $8,12,39-42]$. In this model, the molecule under study (the solute) is treated at the quantum mechanical level and placed inside a cavity made of a set of interlocking spheres generally centered on the atoms, which represents a boundary between the solute and the solvent. The solvent is represented as a continuum medium characterized by a static dielectric constant, $\epsilon$, while the dielectric constant inside the cavity is equal to one. We assume a fast response of the solvent, namely, that the solvent molecules have time to undergo the orientational reorganization induced by the structural changes of the solute in the excited state [1,38, 39,42-44]. Consequently, for water, we employ the static dielectric constant, $\epsilon=78.39$, in all excited-state geometrical optimizations.

\subsubsection{The QMC/PCM approach}

We investigate the effect of PCM solvation on the excited-state geometry within variational Monte Carlo (VMC), using our approach to accurately solve the Poisson equation coupled to a QMC description of the solute [36,37].

Within the PCM, the electric field and the charge density of the solute is coupled to the electric field and the charge density of the solvent via the Poisson equation:

$$
\nabla \cdot \mathbf{E}=4 \pi \rho^{\mathrm{tot}}(\mathbf{r})
$$


where $\mathbf{E}$ is the total electric field and $\rho^{\text {tot }}(\mathbf{r})$ is the total charge density of the electrons and nuclei of the solute and the polarization density of the solvent. In our $\mathrm{VMC} / \mathrm{PCM}$ calculations, we include surface polarization charges on the cavity as well as volume charges in the solvent region to accurately treat solvent polarization effects arising from the quantum mechanical spill-out of the solute electronic density from the cavity.

The volume polarization charge density outside the cavity is given by

$$
\rho_{\mathrm{vol}}^{(\mathrm{pol})}(\mathbf{r})=\left(\frac{1}{\epsilon}-1\right) \rho_{\mathrm{e}}(\mathbf{r})
$$

where $\rho_{\mathrm{e}}(\mathbf{r})$ is the electronic charge density of the solute. The electrostatic potential associated to this charge density is given by the following integral over the domain outside the cavity $\mathcal{C}$ :

$$
\begin{aligned}
\phi_{\mathrm{vol}}^{\mathrm{pol}}(\mathbf{r}) & =\int_{\mathbf{r}^{\prime} \notin \mathcal{C}} \frac{\rho_{\mathrm{vol}}^{\mathrm{pol}}\left(\mathbf{r}^{\prime}\right)}{\left|\mathbf{r}-\mathbf{r}^{\prime}\right|} \mathrm{d} \mathbf{r}^{\prime} \\
& =\left(1-\frac{1}{\epsilon}\right)\left\langle\Psi\left|\sum_{i=1}^{N} \frac{\theta\left(\mathbf{r}_{i}\right)}{\left|\mathbf{r}-\mathbf{r}_{i}\right|}\right| \Psi\right\rangle,
\end{aligned}
$$

where $\Psi$ is the solute wave function and the $\theta$ function is equal to one outside the cavity and zero inside. We employ the VMC approach to compute the integral above by sampling a set of $M$ configurations $\left(\mathbf{r}_{1}^{(k)}, \ldots, \mathbf{r}_{M}^{(k)}\right)$ from the square of the wave function $|\Psi|^{2}$. The electrostatic potential can then be written as due to a set of $n_{c}$ volume polarization charges,

$$
\phi_{\mathrm{vol}}^{\mathrm{pol}}(\mathbf{r}) \approx \sum_{l=1}^{n_{c}} \frac{q_{l}}{\left|\mathbf{r}-\mathbf{r}_{l}\right|},
$$

where the $n_{c} \leq N \times M$ point charges have charge $q_{l}=(1-1 / \epsilon) / M$ and positions corresponding to the sampled one-electron coordinates outside the cavity, $\mathbf{r}_{l}=\mathbf{r}_{i}^{(k)}$ if $\theta\left(\mathbf{r}_{i}^{(k)}\right)=1$.

The surface polarization charge density, $\sigma$, is linked to the total electric field as

$$
\sigma(\mathbf{r})=\frac{1-\epsilon}{4 \pi \epsilon} \mathbf{n}_{+} \cdot \mathbf{E}_{-}(\mathbf{r}),
$$

where $\mathbf{n}_{+}$is a versor pointing outside the cavity and $\mathbf{E}_{-}(\mathbf{r})$ is the total electric field evaluated at the surface immediately inside the cavity, with $\mathbf{E}(\mathbf{r})=\mathbf{E}_{\text {solute }}(\mathbf{r})+$ $\mathbf{E}_{\text {surf }}(\mathbf{r})+\mathbf{E}_{\mathrm{vol}}(\mathbf{r})$. Thus, at the end of a VMC run in the solvent, the normal component of the averaged solute field and of the field produced by volume polarization is computed while, for the field related to the surface polarization charge density, we use the relation

$$
\begin{aligned}
\mathbf{n}_{+} \cdot \mathbf{E}_{\text {surf- }}(\mathbf{r}) & =-2 \pi \sigma(\mathbf{r}) \\
& +\int_{\Sigma} \frac{\mathrm{n}_{+} \cdot\left(\mathbf{r}-\mathbf{r}_{a}\right)}{\left|\mathbf{r}-\mathbf{r}_{a}\right|^{3}} \sigma\left(\mathbf{r}_{a}\right) \mathrm{d} a
\end{aligned}
$$


where the integral is defined over the cavity surface $\Sigma$.

The surface charge distribution is discretized dividing the surface in elements (tesserae) of area $a$ and placing point charges at the center of each of them. In our implementation, the number of point charges per unit surface, $p$, is fixed and it is the same for all the spheres of the cavity. The surface charge density at the point $\mathbf{r}_{\mathbf{k}}$ is then approximated as

$$
\sigma\left(\mathbf{r}_{k}\right) \approx \frac{q_{k}}{a}
$$

where the positions of the point charges are determined by minimizing the mutual repulsion of equal charges on the surface of a sphere of a given fixed radius [45]. The Poisson equation establishes the relation between the surface charge polarization and the nuclear and the electronic density of the solute (which also determines the volume polarization charges) as

$$
q_{k}=\sum_{j} G_{k j}(\Sigma, \epsilon) \mathbf{n}_{+} \cdot\left[\mathbf{E}_{\mathrm{vol}}\left(\mathbf{r}_{j}\right)+\mathbf{E}_{\mathrm{solute}}\left(\mathbf{r}_{j}\right)\right]
$$

where $G_{k j}$ depends only on the shape of the cavity and on the solvent dielectric constant.

This equation must be self-consistently solved with the molecular wave function of the solute, which is optimized in the presence of the PCM solvation through the minimization of the energy functional:

$$
\begin{aligned}
E[\Psi] & =\left\langle\Psi\left|\mathcal{H}_{\text {elec }}\right| \Psi\right\rangle+\sum_{\alpha<\beta} \frac{Z_{\alpha} Z_{\beta}}{\left|\mathbf{R}_{\alpha}-\mathbf{R}_{\beta}\right|} \\
& +\frac{1}{2} \int \rho_{\text {solute }}(\mathbf{r})\left[V_{\sigma}(\mathbf{r})+V_{\text {vol }}(\mathbf{r})\right] \mathrm{d} \mathbf{r},
\end{aligned}
$$

where $\mathcal{H}_{\text {elec }}$ is the electronic Hamiltonian. The density of the solute is given by the sum of the charges of the nuclei $Z_{\alpha}$ at positions $\mathbf{R}_{\alpha}$ and the electronic contribution:

$$
\rho_{\text {solute }}(\mathbf{r})=\sum_{\alpha} Z_{\alpha} \delta\left(\mathbf{r}-\mathbf{R}_{\alpha}\right)+\rho_{e}(\mathbf{r})
$$

and the potential due to the surface and volume charges is given by

$$
V_{\sigma}(\mathbf{r})+V_{\mathrm{vol}}(\mathbf{r})=\sum_{k}^{\text {surf,vol }} \frac{q_{k}}{\left|\mathbf{r}-\mathbf{r}_{\mathbf{k}}\right|} .
$$

The solute-solvent interaction term in the energy functional given in Eq. (4.9) corresponds to the polarization contribution to the free energy of solvation, which we denote as $\Delta G^{(\mathrm{pol})}$ in the following. In order to prevent divergences of the Coulomb interaction at the coalescence points, we introduce a small cutoff distance and, when the electron and the point charge are closer than this distance, we set the interaction equal to the value it takes at the cutoff. 
To describe the solute, we employ a many-body molecular wave function of the Jastrow-Slater form,

$$
\Psi=\mathcal{J} \sum_{i=1}^{N_{\mathrm{CSF}}} c_{i} C_{i}
$$

where $c_{i}$ are the expansion coefficients of the CSFs, $C_{i}$, and $\mathcal{J}$ is the Jastrow correlation factor which has an explicit dependence on the inter-particle distances. At a given geometry of the solute, the wave function is fully optimized by energy minimization within VMC in a state-specific or state-average fashion and brought to self consistence with the surface and volume polarization charges through two cycles of generation of the solvent charges and optimization of the solute wave function for the targeted excited state.

\subsubsection{Computation of the forces in PCM}

The inclusion of the PCM requires however some attention in the computation of the QMC forces by finite difference (as well as analytically) since the surface and volume polarization charges depend on the positions of the nuclear coordinates of the solute. When the atomic displacements are performed to compute the numerical forces, the spheres of the cavity centered on the atoms and, therefore, the point charges on the surface, must be rigidly moved with the atoms when the energy of the solute (Eq. 4.9) is evaluated at the displaced positions. Furthermore, the magnitude of the charges must be modified to account for the change of the electronic density distribution [46].

We give here the details of our procedure and start by considering a small variation of an internal coordinate $x_{\gamma}$ with respect to the starting configuration $\mathbf{x}$, where we have minimized the energy functional (Eq. 4.9) self-consistently with the solvent. The wave function of the solute is recentered and the atomic cavity spheres are rigidly moved with the nuclear centers. The contribution to the forces due to the solvent polarization is then computed as

$$
\frac{\partial \Delta G^{(\mathrm{pol})}}{\partial x_{\gamma}}=\frac{\left[\Delta G^{(\mathrm{pol})}\left(\mathbf{x}+\delta x_{\gamma}\right)-\Delta G^{(\mathrm{pol})}\left(\mathbf{x}-\delta x_{\gamma}\right)\right]}{2 \delta x_{\gamma}}
$$

where $\Delta G^{(\mathrm{pol})}$ consists of two contributions, one from the volume charges and one from the surface charges as described above. The volume polarization charges do not change the value in the molecular deformation but must be repositioned according to the solute wave function recentering. To this end, we resort to the space-warp coordinate transformation of Ref. [47]. Once this operation is complete, the volume contribution to the force is directly evaluated by means of the finite difference.

The surface polarization term is treated differently to avoid the self-consistent recalculation of the surface charges. To explain our procedure, it is convenient to 
rewrite $\Delta G_{\mathrm{surf}}^{(\mathrm{pol})}$ as following:

$$
\begin{aligned}
& \Delta G_{\text {surf }}^{(\text {pol })}=\frac{1}{2} \int \rho_{\text {solute }}(\mathbf{r}) V_{\sigma}(\mathbf{r}) \mathrm{d} \mathbf{r}=\frac{1}{2} \sum_{k} V_{\text {solute }}(\mathbf{r}) q_{k} \\
& =\frac{1}{2} \sum_{k j} V_{\text {solute }}\left(\mathbf{r}_{k}\right) G_{k j} \mathbf{n}_{+} \cdot\left[\mathbf{E}_{\text {vol }}\left(\mathbf{r}_{j}\right)+\mathbf{E}_{\text {solute }}\left(\mathbf{r}_{j}\right)\right],
\end{aligned}
$$

where we used Eqs. 4.8 and 4.9, and $V_{\text {solute }}\left(\mathbf{r}_{k}\right)$ is the solute electrostatic potential at the center of surface element $k$. We can rewrite this equation in matrix form as

$$
\Delta G_{\mathrm{surf}}^{(\mathrm{pol})}=\frac{1}{2} \mathbf{V G E}
$$

where the row vector $\mathbf{V}$ represents the potential of the solute and the column vector $\mathrm{E}$ the electric field normal components. Consequently, Eq. 4.13 becomes

$$
\frac{\partial \Delta G_{\text {surf }}^{(\mathrm{pol})}}{\partial x_{\gamma}}=\frac{\left[\frac{1}{2} \mathbf{V}_{+} \mathbf{G}_{+} \mathbf{E}_{+}-\frac{1}{2} \mathbf{V}_{-} \mathbf{G}_{-} \mathbf{E}_{-}\right]}{2 \delta x_{\gamma}},
$$

where the subscript \pm indicates that the calculation is performed at $\mathbf{x} \pm \delta x_{\gamma}$. In the limit of $\delta x_{\gamma} \rightarrow 0$, we can neglect second and higher order terms in the variation of each quantity depending on $\mathrm{x}$, and write

$$
\begin{aligned}
& \frac{\partial \Delta G_{\text {surf }}^{(\mathrm{pol})}}{\partial x_{\gamma}}=\left[\mathbf{V}_{+} \mathbf{G}_{+} \mathbf{E}_{0}+\mathbf{V}_{0} \mathbf{G}_{0} \mathbf{E}_{+}\right. \\
& \left.-\mathbf{V}_{-} \mathbf{G}_{-} \mathbf{E}_{0}-\mathbf{V}_{0} \mathbf{G}_{0} \mathbf{E}_{-}\right] / 4 \delta x_{\gamma},
\end{aligned}
$$

where the subscript 0 labels quantities evaluated at the initial position $\mathbf{x}$. In order to avoid the recalculation of the electric field at the displaced positions of surface charges, we introduce the following approximation,

$$
\mathbf{V}_{0} \mathbf{G}_{0} \mathbf{E}_{ \pm} \approx \mathbf{V}_{ \pm} \mathbf{G}_{ \pm} \mathbf{E}_{0}
$$

which is motivated by the bilinear form of the energy associated to the electrostatic field. This relation is exact in the case of a solute completely enclosed in a fixed cavity and for the non-discretized treatment of the surface polarization distribution. With this approximation, the above derivative becomes

$$
\frac{\partial \Delta G_{\text {surf }}^{(\mathrm{pol})}}{\partial x_{\gamma}} \approx\left[2 \mathbf{V}_{+} \mathbf{G}_{+} \mathbf{E}_{0}-2 \mathbf{V}_{-} \mathbf{G}_{-} \mathbf{E}_{0}\right] / 4 \delta x_{\gamma}
$$

We are aware that, through this simplification, we are neglecting small contributions of pure geometrical origin. We expect that such effects are generally negligible except for more complicated cavities than those considered in this work, i.e. in case of very large solutes with cavity spheres non centered on nuclei.

To assess the accuracy of our VMC/PCM scheme for the computation of the forces, we investigate the consistency between the energy profile and the computed 
forces for the $\mathrm{LiF}$ molecule, which is a simple system expected to strongly respond to the presence of a polar solvent like water. We employ a single-determinant JastrowSlater wave function and plot the corresponding ground-state energy as a function of
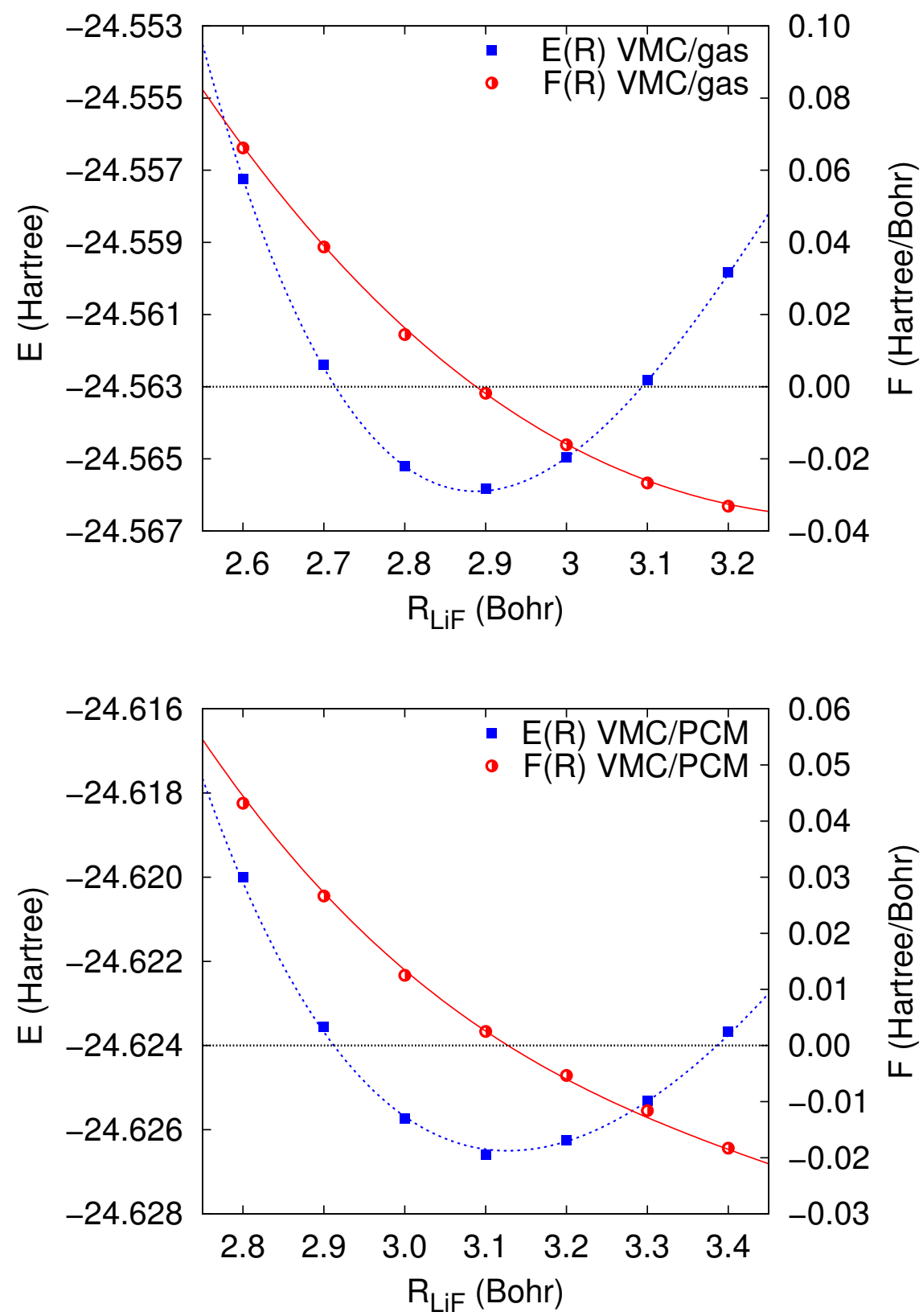

Figure 4.1: Energy (left scale) and force (right scale) of the LiF molecule as a function of the internuclear distance in the gas phase (above) and in PCM water solution (below). The dashed (blue) curve is a polynomial fit to the calculated energies and the solid (red) curve is minus the gradient of the fit. The forces (circles) are calculated as described in the text. The statistical errors are smaller than the symbol size. 
the bond length in the gas phase and in the PCM in Figure 4.1. For each geometry, the wave function is fully optimized in energy minimization and the surface and volume charges of the water environment are determined self-consistently. We then compare the force obtained as minus the derivative of the fit of the energy with the values of the numerical gradients computed with the scheme described above. Clearly, the forces computed at each geometry lie on the curve obtained by differentiating the fit of the energy and the consistency between energy and forces is equally good in the gas phase and in the presence of the PCM. This also demonstrates that the geometrical term we neglect in Eq. 4.19 gives a negligible contribution, smaller than the statistical error on the force. We expect that this approximation is similarly good for the molecules studied here since the shape of their cavities is also rather simple and does not present the complexity one can for instance encounter in the folding of a molecular chain.

\subsection{Computational details}

We perform the TDDFT calculations with the Gaussian09 program [48] and employ the B3LYP [49,50], PBE0 [51-53], M06 [54], M06-2X [54], and CAM-B3LYP [55] exchange-correlation functionals. These functionals represent a subset of those considered in our previous gas-phase study [34] and have also been used in a recent investigation of TDDFT structures in solution [12,13,18-20]. We use the MOLCAS 7.2 suite of programs [56] for the CASPT2 [57,58] calculations, and always employ the recommended zero-order Hamiltonian [27] with the IPEA shift set to 0.25 a.u.

The QMC calculations are carried out with the program package CHAMP [59]. We employ scalar-relativistic energy-consistent Hartree-Fock pseudopotentials [60, 61] and obtain the starting determinantal component of the Jastrow-Slater wave function in complete-active-space self-consistent field (CASSCF) calculations performed with the program GAMESS(US) [62]. The CAS expansions are then expressed on the CASSCF natural orbitals and all configuration state functions (CSF) are retained in the QMC studies of acrolein, MCP, and acetone. For the excited state of the propenic acid anion, the expansion is truncated imposing a threshold of 0.05 on the CSF coefficients and the union of the surviving CSF's in the ground and excited states are then kept in the final wave functions. We employ a three-body Jastrow factor to account for electron-nuclear, electron-electron, and electron-electron-nucleus correlations and use different Jastrow factors to describe different atom types [63]. The difference between the VMC structures optimized with a two-body and a three-body Jastrow factor is rather small, between 0.003-0.006 $\AA$ in the gas phase and 0.003$0.009 \AA$ in the PCM, and a good cancellation of errors is generally observed in the bond-length differences (see Supporting Information). In the following, we present the results obtained with the more sophisticated three-body Jastrow factor. The QMC wave functions are fully optimized within variational Monte Carlo using the linear method [33] and its extension to state-average calculations [21].

We perform the CASPT2 and TDDFT calculations with the Dunning's correlation consistent cc-pVTZ basis set [64-67] and the QMC calculations with the basis 
sets specifically constructed for our pseudopotentials [60]. In particular, in QMC, we employ the cc-pVTZ' basis set which consists of the cc-pVTZ for the heavy atoms and the cc-pVDZ basis set for hydrogen. For these molecules, this choice of basis sets represents a good compromise between accuracy and computational cost in the computation of the bond-length differences between PCM and the gas phase. With respect to the use of augmentation, the largest basis-set error on this difference is observed for the very responsive $\pi \rightarrow \pi^{*}$ state of acrolein and amounts to 0.004 and $0.006 \AA$ at the TDDFT and the CASPT2 level, respectively.

For all PCM calculations, we construct the cavity using interlocking spheres centered on the nuclei, whose radii yield an electronic isodensity surface of 0.001 a.u. In the VMC/PCM calculations, we include both surface and volume polarization charges [36] while the latter are not present in the TDDFT and CASPT2 calculations. In the TDDFT computations, we use the integral equation formalism variant of PCM (IEFPCM) while, in CASPT2, we use the standard PCM implemented in MOLCAS. In the CASPT2/PCM, the surface charges are computed self-consistently in the CASSCF step [41] and then simply included unchanged in the zero-order CASPT2 Hamiltonian. As starting geometry of the PCM excited-state optimizations, we use the corresponding optimal excited-state geometries obtained in the gas phase at the same level of theory.

As detailed in previous work by our group [22,34], the VMC geometry optimization is performed in Z-matrix coordinates with numerical gradients of the energy with respect to the nuclear coordinates. The interatomic forces at a reference geometry are computed in a correlated sampling VMC calculation [47], where a set of secondary geometries is generated through forward and backward displacements of 0.001 a.u. in the bond lengths and 0.01 degrees in the bond and dihedral angles. To obtain finite-variance estimates of the gradients, we sample a distribution which is finite at the origin following Ref. [29]. The VMC/PCM structural optimizations require generally about 5 iterations to converge to a final value of the interatomic gradients of about 0.001-0.003 Hartree/Bohr for the bond lengths and 0.0001 Hartree/degree for the bond angles. We then perform 5-8 additional steps after convergence to obtain the average estimates of the optimal internal coordinates.

\subsection{Results}
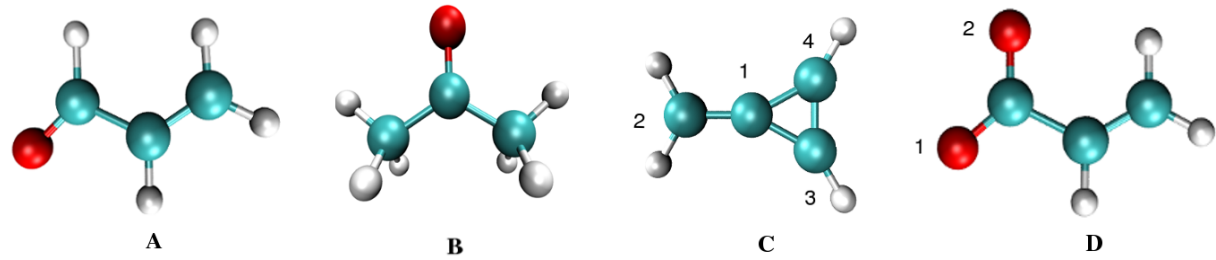

Figure 4.2: The molecules considered in this work: A) $s$-trans acrolein, B) acetone, C) methylenecyclopropene (MCP), and D) the propenoic acid anion (PAA). 
The molecules considered in this work are the $s$-trans acrolein, acetone, methylenecyclopropene (MCP), and the propenoic acid anion (PAA), which are shown in Figure 4.2. The ground- and excited-state properties of these molecules have been extensively investigated in the gas phase with many different quantum chemical methods and are commonly employed to benchmark the quality of the predicted excited-state structures $[15,34,68-77]$. Relatively few studies have instead been performed on the effect of solvation on the ground- and excited-state equilibrium geometries of these molecules $[15-17,19]$ and these studies suffered from the lack good reference data for comparison.

As in our gas-phase study [34], we investigate the optimal structures of the $n \rightarrow$ $\pi^{*}$ state of acrolein, acetone, and PAA, and of the $\pi \rightarrow \pi^{*}$ state of acrolein and MCP, imposing the symmetry constraints detailed in Table 4.1. For these states, we optimize the molecular geometries within VMC and CASPT2 and compare the relative performance of these correlated methods to the TDDFT structures obtained with several approximate exchange-correlation functionals. Here, we will focus on the effect of the solvation PCM environment on the equilibrium geometries, which is quantified in terms of bond-length differences with respect to the corresponding gas-phase structures.

\begin{tabular}{lllc}
\hline Molecule & Symmetry & State & Excitation \\
\hline$s$-trans acrolein & $C_{s}$ & $1^{1} \mathrm{~A}^{\prime \prime}$ & $n \rightarrow \pi^{*}$ \\
& & $2^{1} \mathrm{~A}^{\prime}$ & $\pi \rightarrow \pi^{*}$ \\
Acetone & $C_{2 v}$ & $1^{1} \mathrm{~A}_{2}$ & $n \rightarrow \pi^{*}$ \\
& $C_{s}$ & $1^{1} \mathrm{~A}^{\prime \prime}$ & $n \rightarrow \pi^{*}$ \\
$\mathrm{MCP}$ & $C_{2 v}$ & $1^{1} \mathrm{~B}_{2}$ & $\pi \rightarrow \pi^{*}$ \\
$\mathrm{PAA}$ & $C_{s}$ & $1^{1} \mathrm{~A}^{\prime \prime}$ & $n \rightarrow \pi^{*}$ \\
\hline
\end{tabular}

Table 4.1: Symmetries imposed in the structural optimization of the low-lying excited states of the molecules. The symmetry of the state and the nature of the excitation are also reported.

\subsubsection{Acrolein}

In the CASPT2 and VMC calculations of the $n \rightarrow \pi^{*}$ state of planar acrolein, we use a CAS $(6,5)$ expansion given by four $\pi$ orbitals on the conjugated carbon chain and one $\sigma$ orbital describing a lone pair in the direction orthogonal to the $\mathrm{CO}$ group. In the $\pi \rightarrow \pi^{*}$ state, we employ a CAS $(4,4)$ expansion over four $\pi$ orbitals in VMC and a smaller CAS $(2,2)$ in CASPT2. Both in the gas phase and in PCM, a CASSCF calculations with a CAS $(4,4)$ expansion results in the bright $\pi \rightarrow \pi^{*}$ state being the third root in the $\mathrm{A}^{\prime}$ irreducible representation, which then becomes the second root when dynamical correlation is introduced with the perturbation correction or with the Jastrow factor in VMC. While the VMC optimization with a CAS $(4,4)$ does not pose any problem, the CASPT2 calculations are affected by a strong mixing between the second and third eigenvectors at the multi-state level, and therefore a perturbation wave function dramatically different from the zero-order reference. To better 
illustrate these difficulties, we report the spread of optimal geometries obtained with different active spaces at the single- and multi-state CASPT2 level in the Supporting Information. Therefore, consistently with the choice made in the gas phase, we employ a different active space for the CASPT2 and VMC optimizations of the $\pi \rightarrow \pi^{*}$ structure, keeping in mind that the use of the small CAS $(2,2)$ active space leads to a straightforward CASPT2 optimization of the second state but may be variationally rather poor.

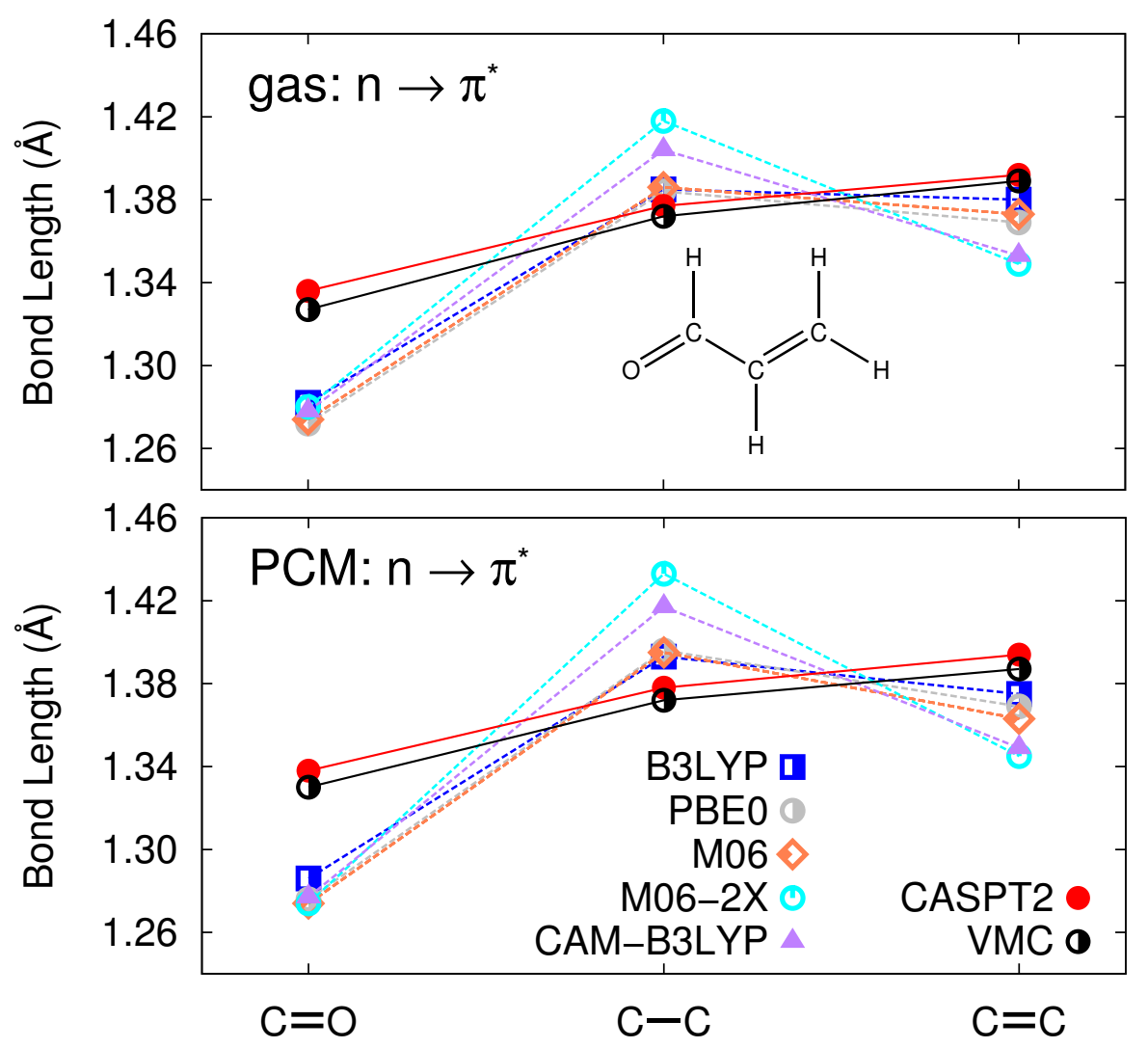

Figure 4.3: Bond lengths ( $\mathrm{A}$ ) in the gas phase (top) and in water solution (bottom) of the $n \rightarrow \pi^{*}$ excited state of $s$-trans acrolein. The statistical errors on the VMC values is about $0.001 \AA$.

The optimal excited-state geometries of $s$-trans acrolein in the gas phase and solvation are shown in Figure 4.3 and Figure 4.4, and the bond-length differences between water and the gas phase are reported in Figure 4.5. In the $n \rightarrow \pi^{*}$ state, we find that VMC and CASPT2 agree in predicting that single and double bonds acquire a more similar length in the excited state and that this pattern is preserved in the PCM environment. The structural response of acrolein to solvation at the correlated level is in fact very small with changes in the bond lengths with respect to the gas phase well below $0.005 \AA$. All TDDFT functionals give instead double bonds in the excited state significantly shorter than the single one, and the difference is enhanced upon solvation: The single $\mathrm{C}-\mathrm{C}$ bond lengthens by about $0.01-0.015 \AA$ with respect to the gas phase, while the effect on the other bonds is much smaller (with the exception 

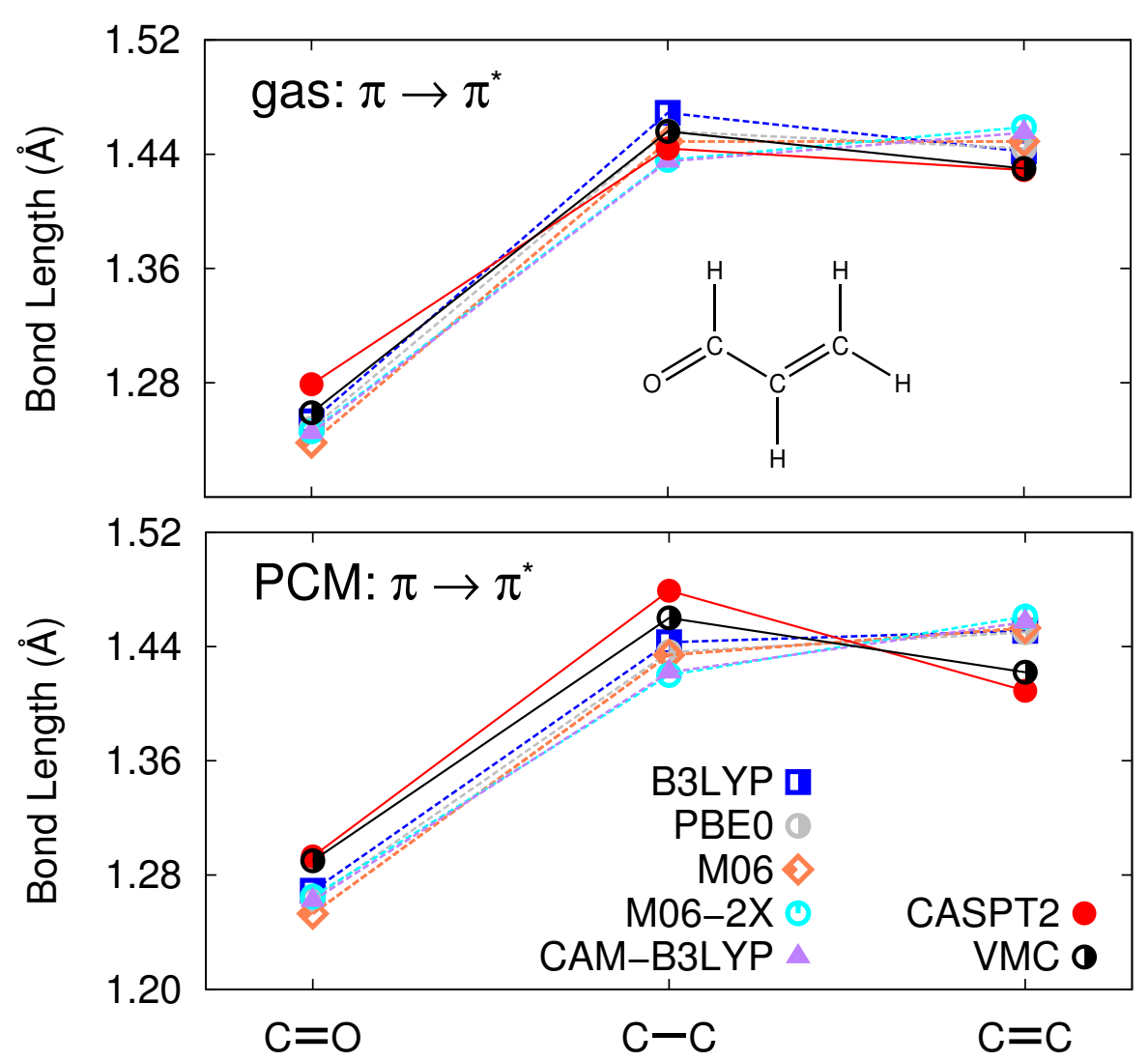

Figure 4.4: Bond lengths ( $($ ) in the gas phase (top) and in water solution (bottom) of the $\pi \rightarrow \pi^{*}$ excited state of $s$-trans acrolein. The statistical error on the VMC values is smaller than $0.001 \AA$.

of the $\mathrm{C}=\mathrm{C}$ bond optimized with the M06 functional which shortens by about 0.01 $\AA$ ). These findings are consistent with a recent TDDFT study [19] where an even less polar solvent than water (acetonitrile) was considered.

The geometry of the $\pi \rightarrow \pi^{*}$ state responds more strongly to the presence of water at the correlated level. This is expected since the dipole moment increases going from the ground to the $\pi \rightarrow \pi^{*}$ excited state, while it decreases in the $n \rightarrow \pi^{*}$ excited state [42]. The VMC and CASPT2 methods predict the same behavior upon solvation, namely, the elongation of the $\mathrm{C}=\mathrm{O}$ and $\mathrm{C}-\mathrm{C}$ bonds, and the shortening of the $\mathrm{C}=\mathrm{C}$ bond. As discussed above, the absolute differences between the two correlated approaches must be attributed to the use of a sub-optimal CAS $(2,2)$ active space for CASPT2 and we will therefore use VMC as reference for the geometry optimization of this state. All TDDFT functionals yield an elongation of the carbonyl bond but display a change opposite to VMC in the carbon-carbon distances, that is, a shortening of the $\mathrm{C}-\mathrm{C}$ bond by about $0.02 \AA$ and a slight lengthening of the $\mathrm{C}=\mathrm{C}$ bond.

Finally, in Figure 4.6, we compare our correlated results with the structural response of SAC-CI and CCSD in PCM water computed in Refs. [15] and [17], respectively. For the $n \rightarrow \pi^{*}$ state, CCSD slightly overestimates the geometrical response 

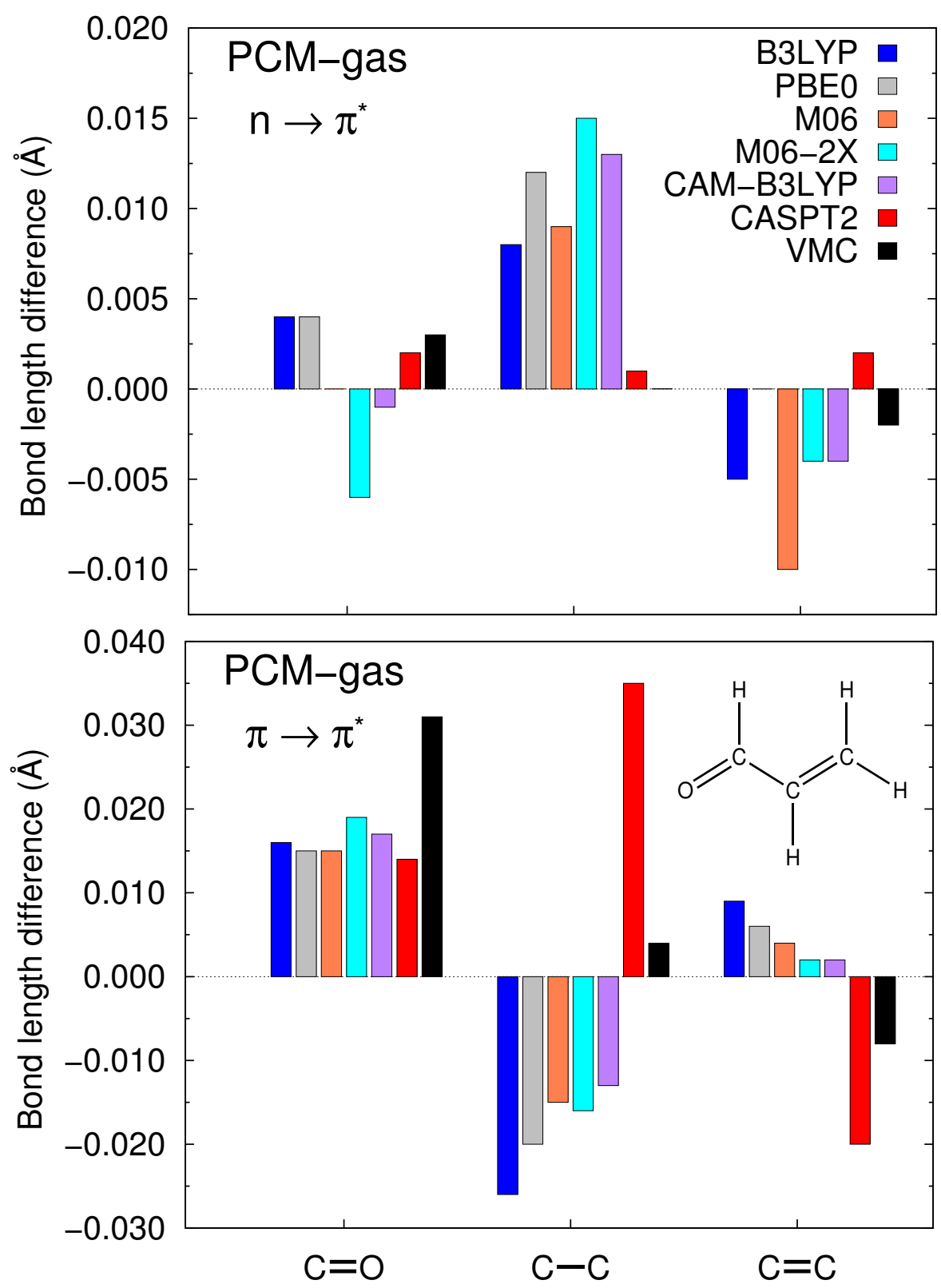

Figure 4.5: Bond-length differences $(\AA)$ between the geometries computed in water solution and in the gas phase for the $n \rightarrow \pi^{*}$ (top) and $\pi \rightarrow \pi^{*}$ (bottom) excited states of $s$-trans acrolein. The statistical error on the VMC bond-length differences is about $0.001 \AA$ for the $n \rightarrow \pi^{*}$ and smaller than $0.001 \AA$ for the $\pi \rightarrow \pi^{*}$ state. The CASPT2 geometry of the $\pi \rightarrow \pi^{*}$ state is obtained with a sub-optimal CAS $(2,2)$ active space (see text).

to the solvent and SAC-CI displays the same trend further amplifying the error with respect to CCSD. In the $\pi \rightarrow \pi^{*}$ state, while SAC-CI and CCSD yield the same pattern as VMC in the bond-length differences between PCM and gas-phase structures, both methods predict an excessive elongation of the $\mathrm{C}-\mathrm{C}$ bond upon solvation by 

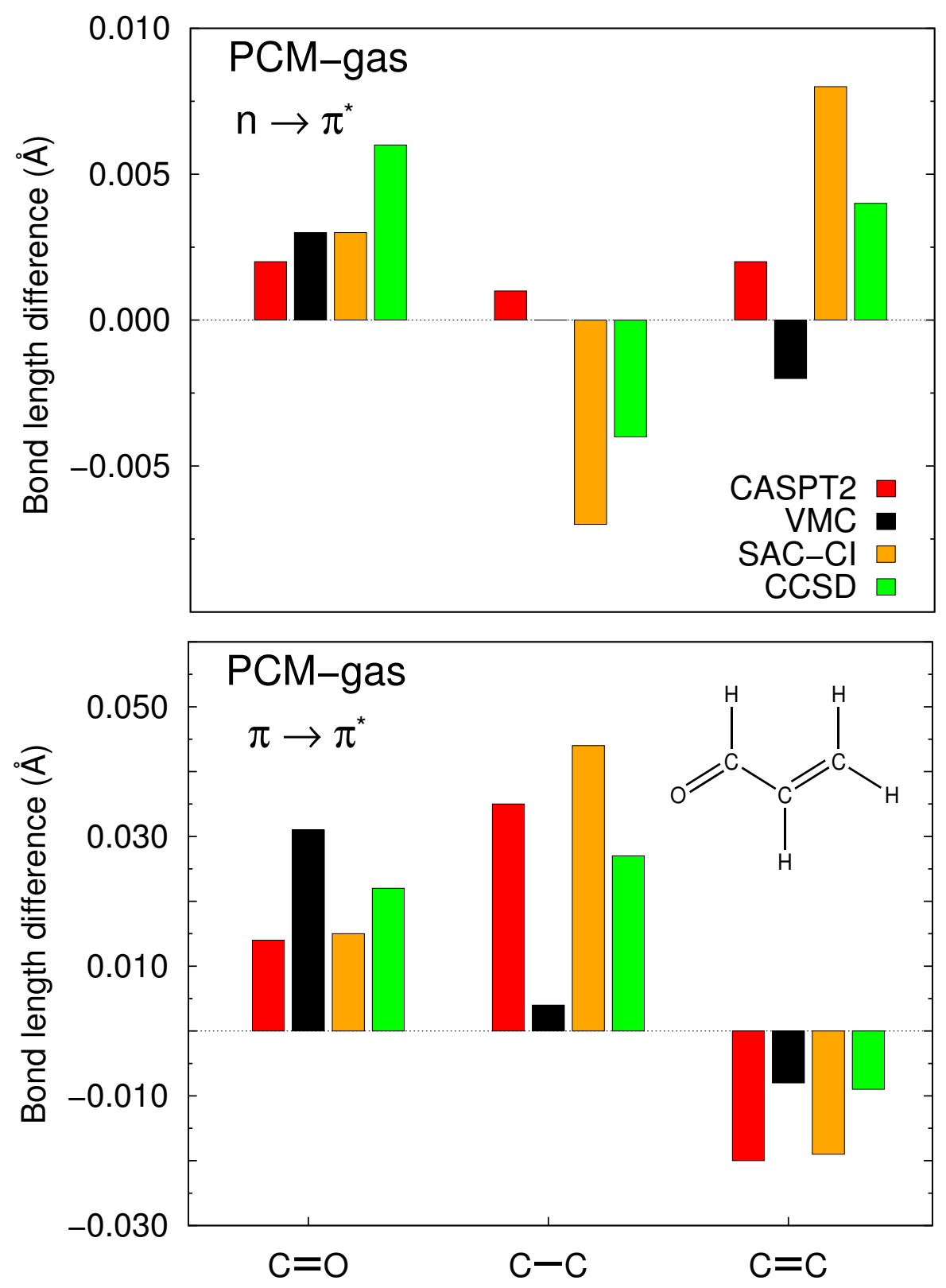

Figure 4.6: CASPT2 and VMC bond-length differences $(\AA)$ between the acrolein geometries computed in water solution and in the gas phase compared with SACCI [15] and CCSD [17] values from the literature. The CASPT2 geometry of the $\pi \rightarrow \pi^{*}$ state is obtained with a sub-optimal CAS $(2,2)$ active space (see text). The statistical error on the VMC bond-length differences is about $0.001 \AA$ for the $n \rightarrow \pi^{*}$ and smaller than $0.001 \AA$ for the $\pi \rightarrow \pi^{*}$ state.

0.04 and $0.03 \AA$, respectively. 


\subsubsection{Acetone}

We optimize the $n \rightarrow \pi^{*}$ excited state of acetone in the $C_{s}$ conformation corresponding to a minimum of the potential energy surface characterized by the pyramidalization of the central carbon atom out of the plane. In the CASPT2 and VMC computations, the active space comprises four electrons in three orbitals, that is, the bonding and antibonding $\pi$ orbitals on the $\mathrm{CO}$ bond, and the nonbonding lone pair on the oxygen.

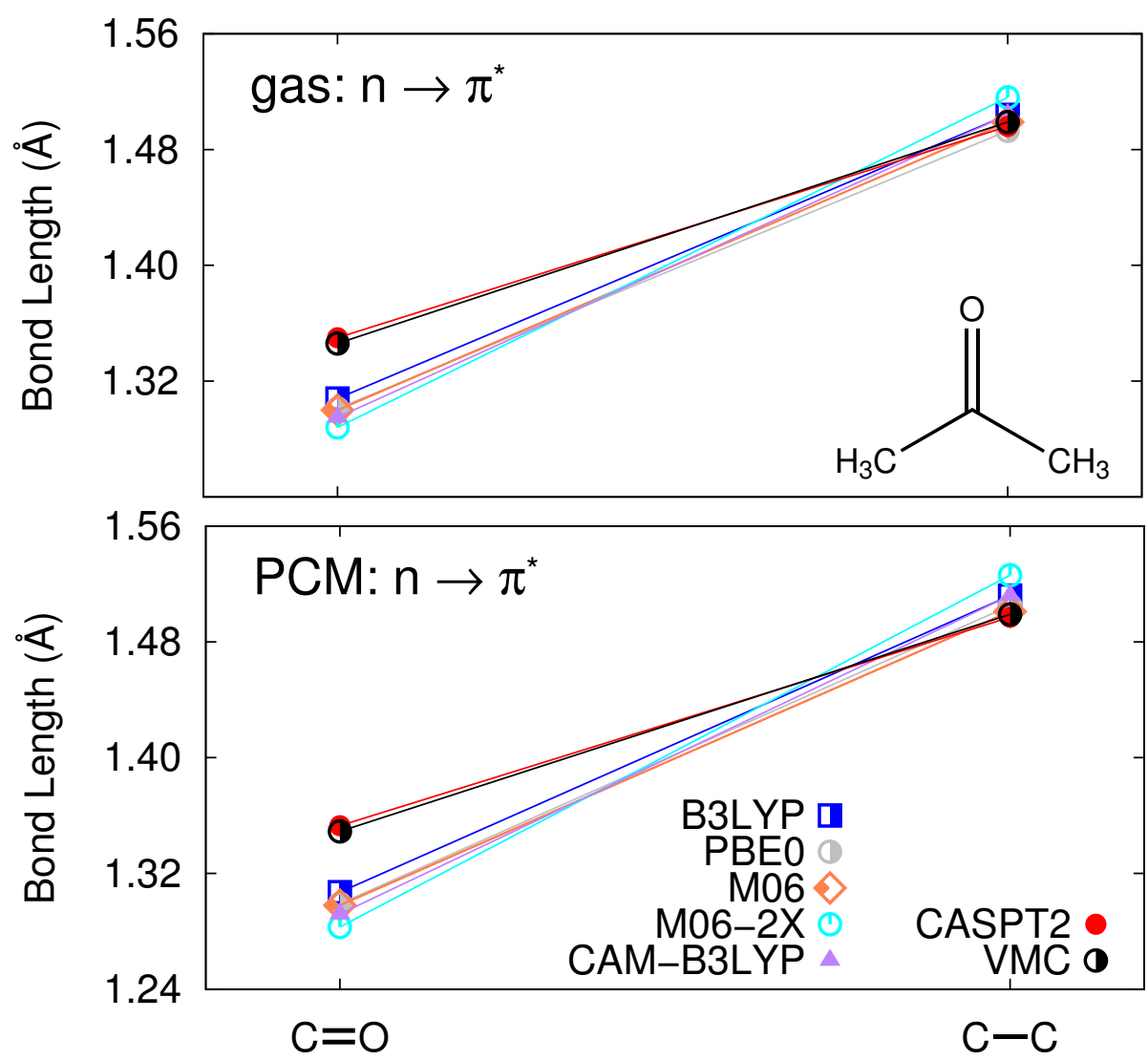

Figure 4.7: Bond lengths ( $\mathrm{A}$ ) in the gas phase (top) and in water solution (bottom) of the $n \rightarrow \pi^{*}$ state of acetone. The statistical error on the VMC values is smaller than $0.001 \AA$.

The excited-state geometries optimized in the gas phase and in water solution are shown in Figure 4.7 and the corresponding bond-length differences in Figure 4.8. All TDDFT functionals yield an optimal solvated structure with the carbonyl bond slightly shorter than in the gas phase and with the CC bonds longer by about 0.005 $0.01 \AA$ depending on the functional. As in the case of the $n \rightarrow \pi^{*}$ state of acrolein, CASPT2 and VMC display a different response to solvation than TDDFT yielding no appreciable changes in the optimized geometries with respect to the gas phase: The VMC bond-length difference on the CC bond is zero within statistical error and on the CO bond below $0.005 \AA$, consistently with CASPT2. 


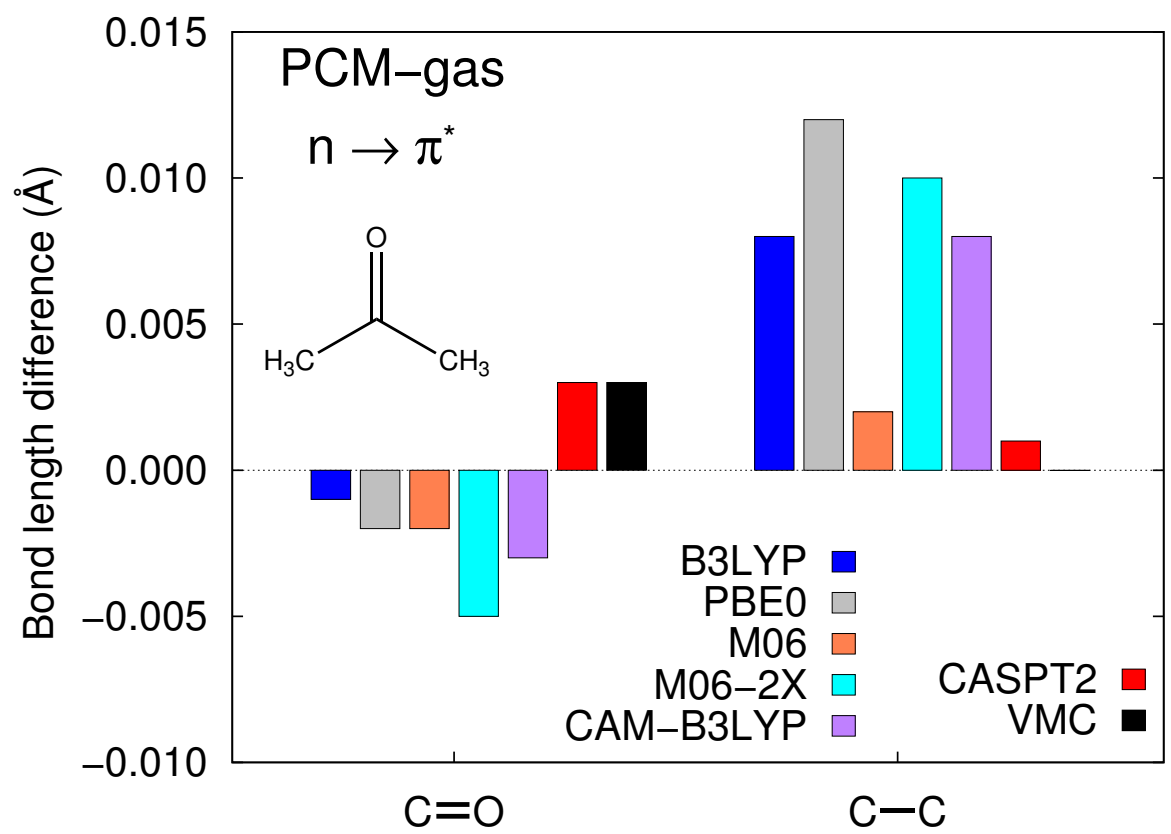

Figure 4.8: Bond length differences $(\AA)$ between the geometries computed in water solution and in the gas phase for the $n \rightarrow \pi^{*}$ state of acetone. The statistical error on the VMC bond-length differences is smaller than $0.001 \AA$.

\subsubsection{Methylenecyclopropene}

In planar MCP, the dipole moment in the $\pi \rightarrow \pi^{*}$ excited state lies along the $\mathrm{C}_{1}=\mathrm{C}_{2}$ bond and has similar magnitude but opposite directions than in the ground state. More precisely, the dipole moment points towards the exocyclic bond in the ground state and towards the ring in the excited state, reflecting a flux of charge in the same direction upon excitation. This remarkable variation in the distribution of the electronic density leads to the simultaneous shortening of the $\mathrm{C}_{1}=\mathrm{C}_{2}$ bond and the lengthening of the $\mathrm{C}_{1}-\mathrm{C}_{3}$ and $\mathrm{C}_{3}=\mathrm{C}_{4}$ bonds in the excited state $[34,42]$. The $\pi \rightarrow \pi^{*}$ excited state of MCP in water is optimized maintaining planarity and using at the correlated level a CAS $(4,4)$ reference wave function which includes the two bonding and two antibonding $\pi$ orbitals.

As shown in Figure 4.9 and Figure 4.10, the effect of introducing a solvation environment is rather small and of the order of mA both in VMC and in CASPT2. All TDDFT functionals predict instead that the change in the bond-length pattern observed upon excitation in the gas phase is emphasized in water: The $\mathrm{C}_{1}=\mathrm{C}_{2}$ bond becomes consistently shorter by about $0.015 \AA$, while the $\mathrm{C}_{1}-\mathrm{C}_{3}$ and $\mathrm{C}_{3}=\mathrm{C}_{4}$ bonds are slightly elongated. Finally, we compare the CASPT2 and VMC bond-length differences with the SAC-CI values from Ref. [15] in Figure 4.11. Again, we find that SAC-CI yields significant deviations on the excited-state structures not only in the gas phase [34] but also in response to solvation. 

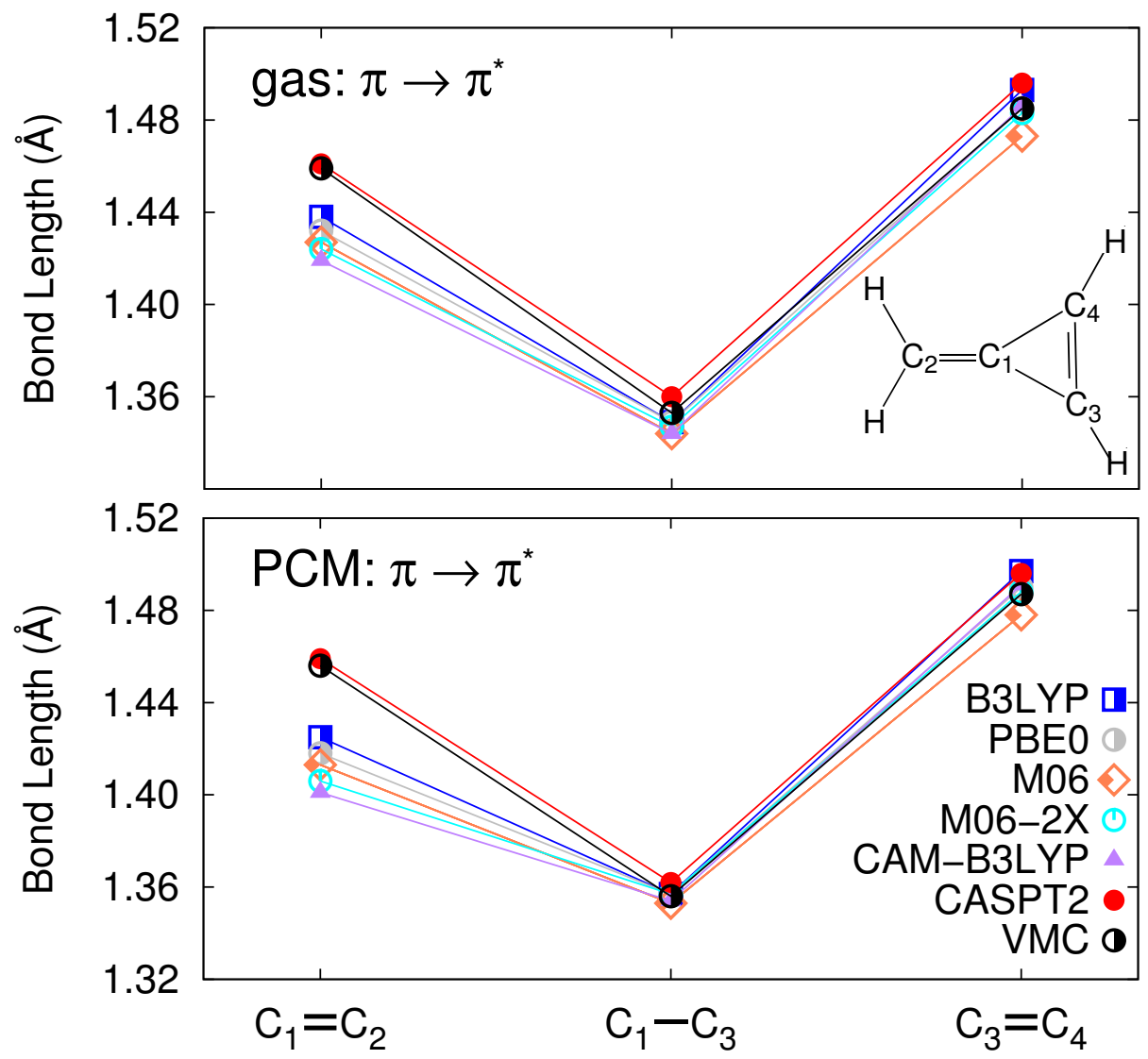

Figure 4.9: Bond lengths ( $⿱$ ) $)$ in the gas phase (top) and in water solution (bottom) of the $\pi \rightarrow \pi^{*}$ state of MCP. The statistical error on the VMC values is smaller than $0.001 \AA$. 


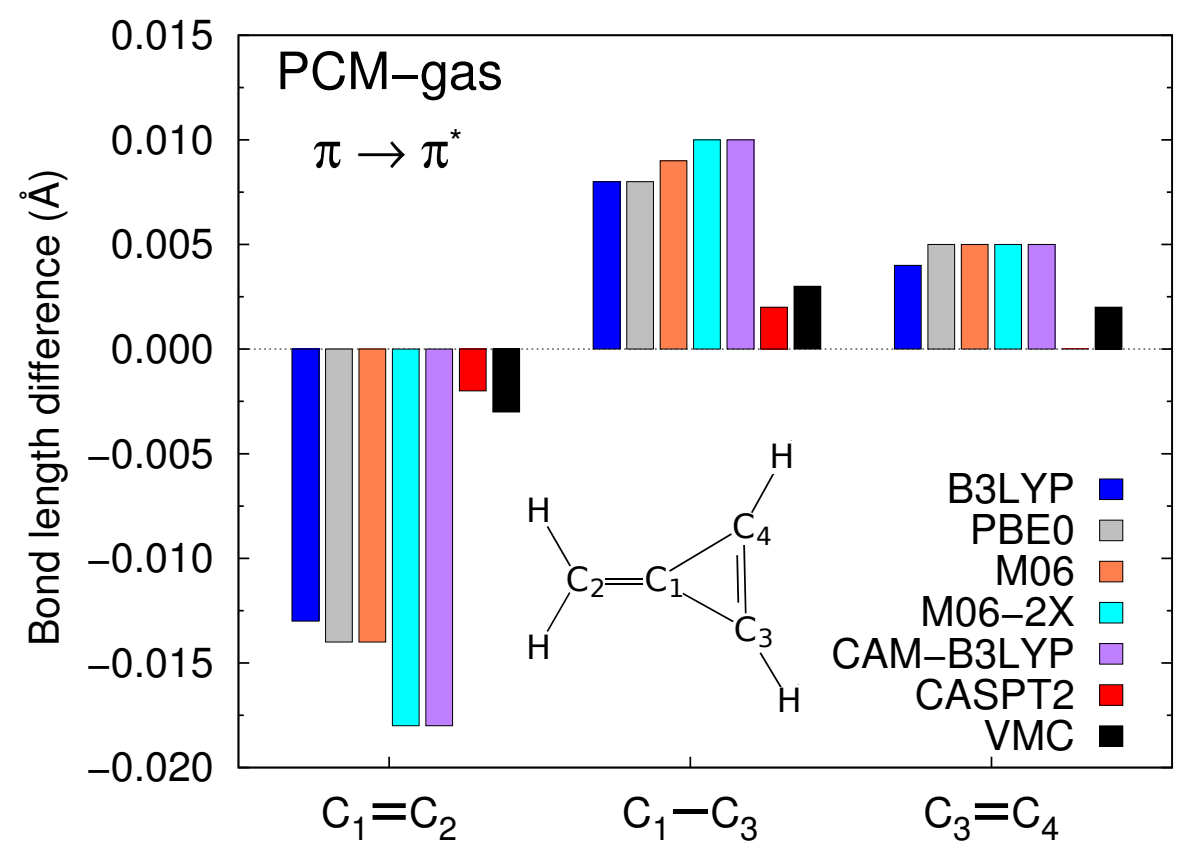

Figure 4.10: Bond-length differences $(\AA)$ between the geometries computed with in water solution and in the gas phase for the $\pi \rightarrow \pi^{*}$ state of MCP. The statistical error on the VMC bond-length differences is smaller than $0.001 \AA$.

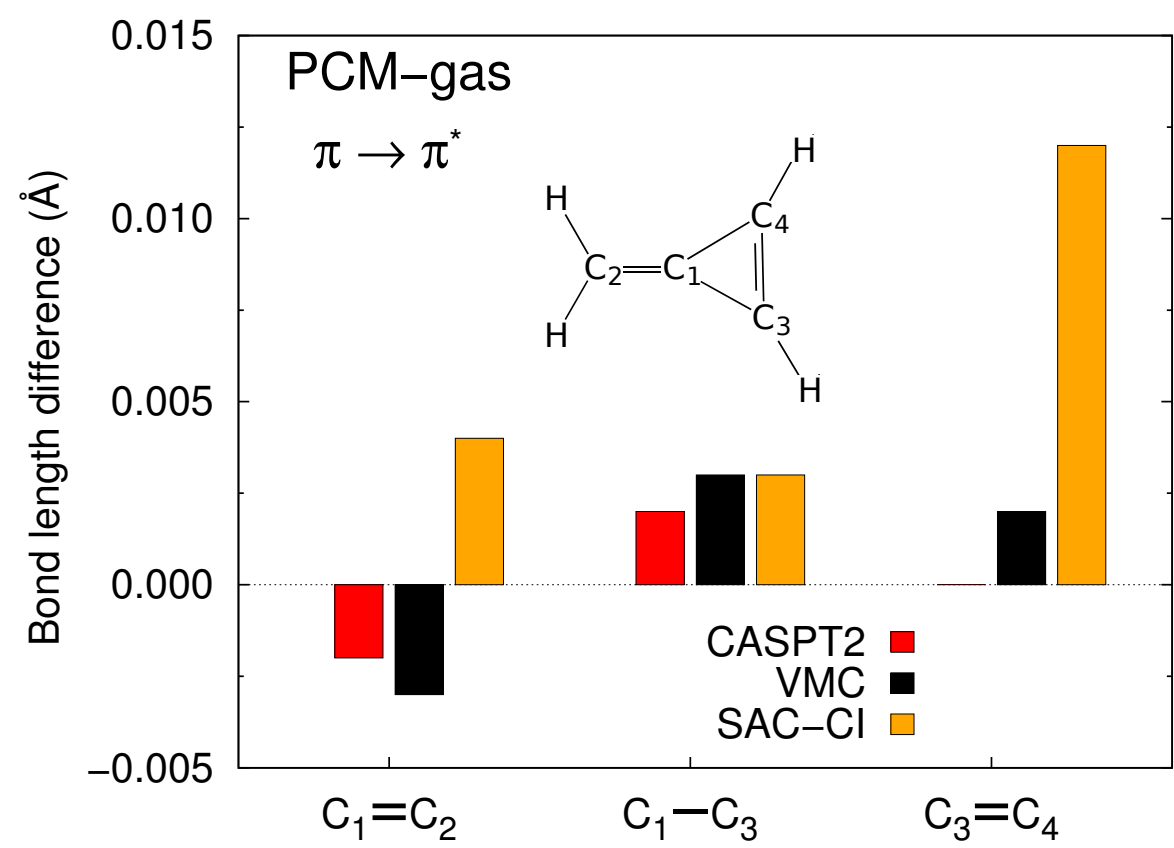

Figure 4.11: CASPT2 and VMC bond-length differences $(\AA)$ between the geometries computed in water solution and in the gas phase for the $\pi \rightarrow \pi^{*}$ state of MCP, compared with the SAC-CI values from Ref. [15]. The statistical error on the VMC bond-length differences is smaller than $0.001 \AA$. 


\subsubsection{Propenoic acid anion}

The last molecule considered in this work is the propenoic acid anion in its $n \rightarrow$ $\pi^{*}$ excited state. For the CASPT2 and VMC optimizations, we use a CAS $(10,7)$ expansion, which includes five $\pi$ orbitals on the conjugated chain and two $\sigma$ orbitals on the oxygen atoms to describe the lone pairs. We employed this same active space in the gas-phase optimizations of our previous study [34], where we showed how the choice of a smaller active space with only one of the two lone pairs leads to excited-state geometries with a differential elongation of the $\mathrm{C}-\mathrm{O}_{1}$ and $\mathrm{C}-\mathrm{O}_{2}$ bonds, depending on which lone pair is added to the active space.

The excited-state geometries of PAA in water and in the gas phase, and their difference are shown in Figure 4.12 and Figure 4.13. The effect of PCM on the CASPT2 geometries is to slightly shorten all bonds with respect to the gas phase, while the VMC response to the presence of the solvent is almost zero. As in the case of the other molecules, the response of TDDFT is stronger with the CC bonds shortening by 0.01-0.02 and the CO bonds lengthening by about 0.01-0.02 $\AA$. These results are consistent with what reported in a recent TDDFT structural study in a solvated PCM environment [19].
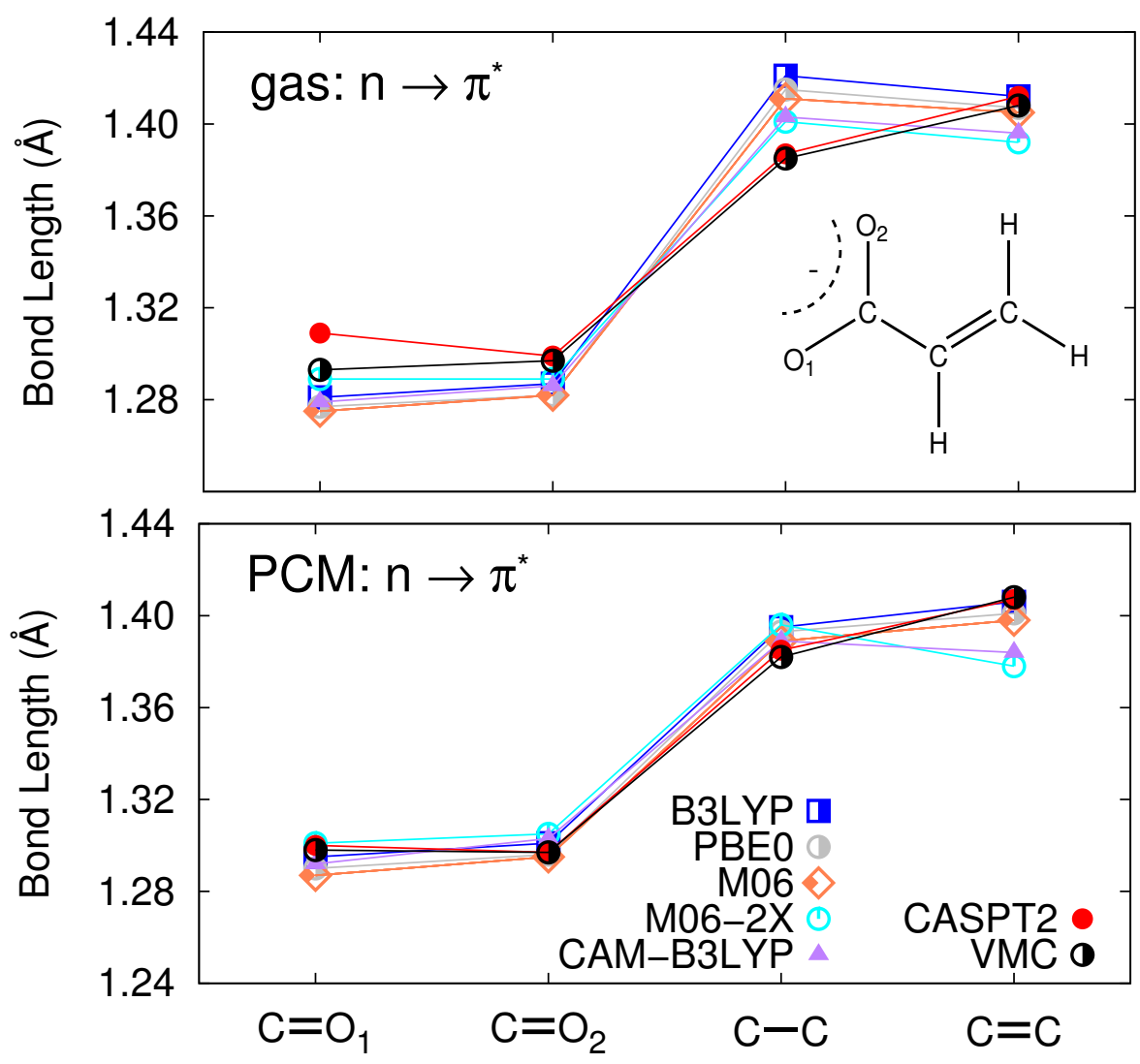

Figure 4.12: Bond lengths ( $\mathrm{\AA}$ ) in the gas phase (top) and in water solution (bottom) of the $n \rightarrow \pi^{*}$ state of PAA. The statistical error on the VMC values is smaller than $0.001 \AA$. 


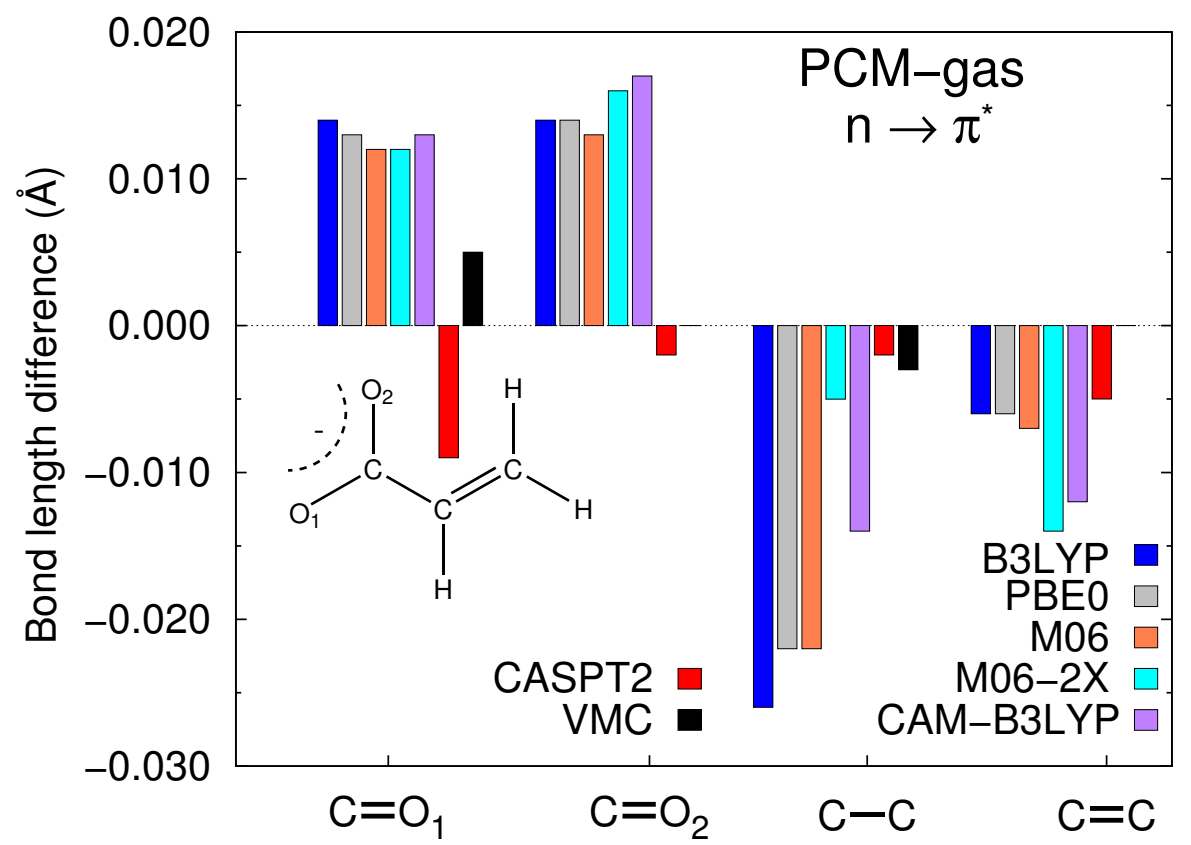

Figure 4.13: Bond-length differences $(\AA)$ between the geometries computed in water solution and in the gas for the $n \rightarrow \pi^{*}$ state of PAA. The statistical error on the VMC bond-length differences is smaller than $0.001 \AA$.

\subsection{Conclusions}

This work combines for the first time the PCM model of solvation with QMC techniques to perform geometry optimizations in the ground and excited states. We consider here a set of of chromophores in water representative of $n \rightarrow \pi^{*}$ and $\pi \rightarrow \pi^{*}$ excitations, and compute the optimal excited-state geometries with the QMC method in its simplest VMC variant, coupled to the PCM description of the solvent. Importantly, in our implementation of the PCM model, the reaction field includes both volume and surface polarization charges and is determined self consistently with the molecular wave function during the QMC optimization of the solute geometry. These features must be contrasted to common implementations of the PCM model where the volume charges are not introduced or the response of the reaction field is not taken into account at the same level of theory as in the CASPT2 calculations. The consistency between the energy profile and the forces computed with our VMC/PCM approach is demonstrated for the test case of $\mathrm{LiF}$, a simple molecule whose structure strongly responds to a polar solvent like water.

To establish the robustness of our scheme as well as offer reliable reference data for TDDFT in solution, we compare the excited-state QMC geometries of the selected chromophores in water to the structures we optimized with CASPT2 and a variety of TDDFT functionals. The geometrical response to the solvent estimated in QMC is found to be in very good agreement with the predictions of the CASPT2 method, with the only exception of the $\pi \rightarrow \pi^{*}$ state of acrolein where one must use the computational expedient of a sub-optimal active space at the perturbation level 
while no such compromise is needed in the QMC optimization of this excited state.

As regards TDDFT, we find that the functionals investigated here generally overestimate the geometrical variations in the excited states due to the presence of the solvent, further increasing the discrepancy with the correlated approaches already observed in the gas phase. The $\pi \rightarrow \pi^{*}$ excited state of acrolein is the only state which sees a non-negligible structural response to solvation also at the correlated level with QMC bond-length differences with respect to the gas phase as large as $0.03 \AA$ A. However, while VMC and CASPT2 predict the elongation of the $\mathrm{C}-\mathrm{C}$ and the shortening of the $\mathrm{C}=\mathrm{C}$ bond in solution, all TDDFT functionals yield the opposite behavior.

In conclusion, we have demonstrated that QMC in its simplest VMC flavor is an accurate method for structural optimization in the presence of PCM solvation. Thanks to its favorable computational scaling as compared to other correlated approaches, our QMC scheme self-consistently coupled to the reaction field via surface and volume charges is therefore a robust and promising approach to investigate the structural relaxation and fluorescence in solution of also larger molecules with higher accuracy than present TDDFT functionals. 


\section{Bibliography}

[1] J. Tomasi, B. Mennucci, and R. Cammi, Chem. Rev. 105, 2999 (2005).

[2] H. Lin and D. Truhlar, Theor. Chem. Acc. 117, 185 (2007).

[3] H. M. Senn and W. Thiel, Angew. Chem. Int. Ed. 48, 1198 (2009).

[4] G. Seabra, J. Swails, and A. Roitberg, in Multi-Scale Quantum Models for Biocatalysis, Vol. 7 of Challenges and Advances in Computational Chemistry and Physics, edited by D. York and T.-S. Lee (PUBLISHER, Springer: New York, 2009), pp. 3-20.

[5] B. Mennucci, Phys. Chem. Chem. Phys. 15, 6583 (2013).

[6] J. Tomasi and M. Persico, Chem. Rev. 94, 2027 (1994).

[7] V. Barone and M. Cossi, J. Phys. Chem. A 102, 1995 (1998).

[8] D. M. Chipman, J. Chem. Phys. 131, 014103 (2009).

[9] M. Casida, J. Mol. Struc. (Theochem) 914, 3 (2009).

[10] M. Casida, M. E.and Huix-Rotllant, Annu. Rev. Phys. Chem. 63, 287 (2012).

[11] M. Marques, T. N. Maitra, F. M. S. Nogueira, E. Gross, and R. A., Fundamentals of Time-Dependent Density Functional Theory (Springer, Heidelberg, 2012), pp. 53-99.

[12] G. Scalmani, J. M. Frisch, B. Mennucci, J. Tomasi, R. Cammi, and V. Barone, J. Chem. Phys. 124, 094107 (2006).

[13] D. Jacquemin, E. A. Perpète, X. Assfeld, G. Scalmani, M. J. Frisch, and C. Adamo, Chem. Phys. Lett. 438, 208 (2007).

[14] O. Clemens, M. Basters, M. Wild, S. Wilbrand, C. Reichert, M. Bauer, M. Springborg, and G. Jung, J. Mol. Struc. (Theochem) 866, 15 (2008).

[15] R. Cammi, R. Fukuda, M. Ehara, and H. Nakatsuij, J. Chem. Phys. 133, 024104 (2010).

[16] M. Caricato, J. Chem. Theory Comput. 8, 4494 (2012). 
[17] M. Caricato, J. Chem. Theory Comput. 8, 5081 (2012).

[18] D. Jacquemin, A. Planchat, C. Adamo, and B. Mennucci, J. Chem. Theory Comput. 8, 2359 (2012).

[19] C. A. Guido, S. Knecht, J. Kongsted, and B. Mennucci, J. Chem. Theory Comput. 9, 2209 (2013).

[20] S. Chibani, A. D. Laurent, A. Blondel, B. Mennucci, and D. Jacquemin, J. Chem. Theory Comput. 10, 1848 (2014).

[21] C. Filippi, M. Zaccheddu, and F. Buda, J. Chem. Theory Comput. 5, 2074 (2009).

[22] O. Valsson and C. Filippi, J. Chem. theory Comput. 6, 1275 (2010).

[23] R. Send, O. Valsson, and C. Filippi, J. Chem. Theory Comput. 7, 444 (2011).

[24] C. Filippi, F. Buda, L. Guidoni, and A. Sinicropi, J. Chem. Theory and Comput. 8, 112 (2012).

[25] O. Valsson, C. Angeli, and C. Filippi, Phys. Chem. Chem. Phys. 14, 11015 (2012).

[26] O. Valsson, P. Campomanes, I. Tavernelli, U. Rothlisberger, and C. Filippi, J. Chem. Theory and Comput. 9, 2441 (2013).

[27] G. Ghigo, B. O. Roos, and P.-Å. Malmqvist, Chem. Phys. Lett. 396, 142 (2004).

[28] C. Angeli, R. Cimiraglia, S. Evengelisti, T. Leininger, and J.-P. Malrieu, J. Chem. Phys. 114, 10252 (2001).

[29] C. Attaccalite and S. Sorella, Phys. Rev. Lett. 100, 114501 (2008).

[30] S. Sorella and L. Capriotti, J. Chem. Phys. 133, 234111 (2010).

[31] M. Barborini and L. Guidoni, J. Chem. Phys. 137, 224309 (2012).

[32] S. Saccani, C. Filippi, and S. Moroni, J. Chem. Phys. 138, 084109 (2013).

[33] C. J. Umrigar, J. Toulouse, C. Filippi, S. Sorella, and R. G. Hennig, Phys. Rev. Lett. 98, 110201 (2007).

[34] R. Guareschi and C. Filippi, J. Chem. Theory Comput. 9, 5513 (2013).

[35] C. Amovilli, C. Filippi, and F. M. Floris, J. Phys. Chem. B 110, 26225 (2006).

[36] C. Amovilli, C. Filippi, and F. M. Floris, J. Chem. Phys. 129, 244106 (2008).

[37] F. M. Floris, C. Filippi, and C. Amovilli, J. Chem. Phys. 137, 075102 (2012).

[38] F. M. Floris, C. Filippi, and C. Amovilli, J. Chem. Phys. 140, 034109 (2014). 
[39] R. Cammi and B. Mennucci, J. Chem. Phys. 110, 9877 (1999).

[40] M. Cossi and V. Barone, J. Chem. Phys. 115, 4708 (2001).

[41] M. Cossi, V. Barone, N. Rega, G. Scalmani, and V. Barone, J. Chem. Phys. 114, 5691 (2001).

[42] M. Caricato, B. Mennucci, J. Tomasi, F. Ingrosso, R. Cammi, S. Corni, and G. Scalmani, J. Chem. Phys. 124, 124520 (2006).

[43] D. Jacquemin, A. Planchat, C. Adamo, and B. Mennucci, J. Chem. Theory Comput. 8, 2359 (2012).

[44] D. Jacquemin, B. Mennucci, and C. Adamo, Phys. Chem. Chem. Phys. 13, 16987 (2011).

[45] C. Amovilli and N. H. March, Chem. Phys. Lett. 347, 459 (2008).

[46] R. Cammi and J. Tomasi, J. Chem. Phys. 101, 3888 (1994).

[47] C. Filippi and C. J. Umrigar, Phys. Rev. B 61, R16291 (2000).

[48] Gaussian 09 Revision A.02, M. J. Frisch, G. W. Trucks, H. B. Schlegel, G. E. Scuseria, M. A. Robb, J. R. Cheeseman, G. Scalmani, V. Barone, B. Mennucci, G. A. Petersson, H. Nakatsuji, M. Caricato, X. Li, H. P. Hratchian, A. F. Izmaylov, J. Bloino, G. Zheng, J. L. Sonnenberg, M. Hada, M. Ehara, K. Toyota, R. Fukuda, J. Hasegawa, M. Ishida, T. Nakajima, Y. Honda, O. Kitao, H. Nakai, T. Vreven, J. A. Montgomery, Jr., J. E. Peralta, F. Ogliaro, M. Bearpark, J. J. Heyd, E. Brothers, K. N. Kudin, V. N. Staroverov, R. Kobayashi, J. Normand, K. Raghavachari, A. Rendell, J. C. Burant, S. S. Iyengar, J. Tomasi, M. Cossi, N. Rega, J. M. Millam, M. Klene, J. E. Knox, J. B. Cross, V. Bakken, C. Adamo, J. Jaramillo, R. Gomperts, R. E. Stratmann, O. Yazyev, A. J. Austin, R. Cammi, C. Pomelli, J. W. Ochterski, R. L. Martin, K. Morokuma, V. G. Zakrzewski, G. A. Voth, P. Salvador, J. J. Dannenberg, S. Dapprich, A. D. Daniels, Ö. Farkas and J. B. Foresman, J. V. Ortiz, J. Cioslowski, and D. J. Fox, Gaussian Inc. Wallingford CT 2009.

[49] A. D. Becke, J. Chem. Phys. 98, 5648 (1993).

[50] P. J. Stephens, F. J. Devlin, C. F. Chabalowski, and M. J. Frisch, J. Phys. Chem. 98, 11623 (1994).

[51] J. P. Perdew, K. Burke, and M. Ernzerhof, J. Chem. Phys. 105, 9982 (1996).

[52] C. Adamo and V. Barone, J. Chem. Phys. 110, 6158 (1999).

[53] M. Ernzerhof and G. E. Scuseria, J. Chem. Phys. 110, 5029 (1999).

[54] Y. Zhao and D. Truhlar, Theor. Chem. Acc. 120, 215 (2008). 
[55] T. Yanai, D. P. Tew, and N. C. Handy, Chem. Phys. Lett. 393, 51 (2004).

[56] G. Karlström, R. Lindh, P.-Å. Malmqvist, B. O. Roos, U. Ryde, V. Veryazov, P.O. Widmark, M. Cossi, B. Schimmelpfennig, P. Neogrady, and L. Seijo, Comput. Mater. Sci. 28, 222 (2003).

[57] K. Andersson, P.-A. Malmqvist, B. O. Roos, A. J. Sadlej, and K. Wolinski, J. Phys. Chem. 94, 5483 (1990).

[58] K. Andersson, P.-A. Malmqvist, and B. O. Roos, J. Chem. Phys. 96, 1218 (1992).

[59] CHAMP is a quantum Monte Carlo program package written by C. J. Umrigar, C. Filippi and collaborators.

[60] M. Burkatzki, C. Filippi, and M. Dolg, J. Chem. Phys. 126, 234105 (2007).

[61] For the hydrogen atom, we use a more accurate BFD pseudopotential and basis set. Dolg, M.; Filippi, C., private communication.

[62] M. W. Schmidt, K. K. Baldridge, J. A. Boatz, S. T. Elbert, M. S. Gordon, J. H. Jensen, S. Koseki, N. Matsunaga, K. A. Nguyen, S. Su, T. L. Windus, M. Dupuis, and J. A. M. Jr, J. Comput. Chem. 14, 1347 (1993).

[63] C. Filippi and C. J. Umrigar, J. Chem. Phys. 105, 213 (1996), as Jastrow correlation factor, we use the exponential of the sum of three fifth-order polynomials of the electron-nuclear (e-n), the electron-electron (e-e). The Jastrow factor is adapted to deal with pseudo-atoms, and the scaling factor $\kappa$ is set to 0.6 a.u.The 2-body Jastrow factor includes five parameters in the e-e terms and four parameters for each atom type in the e-n terms.

[64] T. H. Dunning Jr, J. Chem. Phys. 90, 1007 (1989).

[65] K. A. Peterson, D. E. Woon, and T. H. Dunning Jr, J. Chem. Phys. 100, 7410 (1994).

[66] A. Wilson, T. van Mourik, and T. H. Dunning Jr, J. Mol. Struct. (Theochem) 388, 339 (1997).

[67] F. Weigend, Phys. Chem. Chem. Phys. 8, 1057 (2006).

[68] M. Reguero, M. Olivucci, F. Bernardi, and M. A. Robb, J. Am. Chem. Soc. 116, 2103 (1994).

[69] S. R. Gwaltney and R. J. Bartlett, J. Chem. Phys. 110, 62 (1999).

[70] F. Aquilante, V. Barone, and B. Roos, J. Chem. Phys. 119, 12323 (2003).

[71] S. C. Page and M. Olivucci, J. Comput. Chem. 24, 298 (2003). 
[72] C. Angeli, S. Borini, L. Ferrighi, and R. Cimiraglia, J. Chem. Phys. 122, 114304 (2005).

[73] C. Angeli, S. Borini, L. Ferrighi, and R. Cimiraglia, J. Mol. Struct. (Theochem) 718, 55 (2005).

[74] B. Saha, M. Ehara, and H. Nakatsuji, J. Chem. Phys. 125, 014316 (2006).

[75] A. M. Losa, I. F. Galván, M. A. Aguilar, and M. E. Martín, J. Phys. Chem. B 111, 9864 (2007).

[76] C. A. Guido, D. Jacquemin, C. Adamo, and B. Mennucci, J. Phys. Chem. A 114, 13402 (2010).

[77] I. Gadaczeck, K. Krause, K. J. Hintze, and T. Bredow, J. Chem. Theory Comput. 8, 986 (2012). 


\section{Chapter 5}

\section{Introducing QMC/MMpol: Quantum Monte Carlo in polarizable force fields for excited states $^{\dagger}$}

We present for the first time a quantum mechanics/molecular mechanics scheme which combines quantum Monte Carlo with the reaction field of classical polarizable dipoles (QMC/MMpol). In our approach, the optimal dipoles are self-consistently generated at the variational Monte Carlo level and then used to include environmental effects in diffusion Monte Carlo. We investigate the performance of this hybrid model in describing the vertical excitation energies of prototypical small molecules solvated in water, namely, methylenecyclopropene and $s$-trans acrolein. Two polarization regimes are explored where either the dipoles are optimized with respect to the ground-state solute density (polGS) or different sets of dipoles are separately brought to equilibrium with the states involved in the electronic transition (polSS). By comparing with reference supermolecular calculations where both solute and solvent are treated quantum mechanically, we find that the inclusion of the response of the environment to the excitation of the solute leads to superior results than the use of a frozen environment (point charges or polGS), in particular, when the solute-solvent coupling is dominated by electrostatic effects which are well recovered in the polSS condition. QMC/MMpol represents therefore a robust scheme to treat important environmental effects beyond static point charges, combining the accuracy of QMC with the simplicity of a classical approach.

$\dagger$ This chapter has been published as R. Guareschi, H. Zulfikri, C. Daday, F. M. Floris, C. Amovilli, B. Mennucci, and C. Filippi, "Introducing QMC/MMpol: Quantum Monte Carlo in polarizable force fields for excited states", J. Chem. Theory Comput. 2016, 12, 1674-1683 


\subsection{Introduction}

The effective treatment of complex processes of chemical and biological relevance imposes practical choices in the construction of the theoretical model. The system is divided into an active region and the surrounding environment, and the two parts are treated at a different level of accuracy. A liquid solution is a typical application of such an approach: One or more active molecules (the solute) are embedded in an external potential generated by the solvent. Within this framework, the challenge is to combine the accuracy of the model with the increasing computational requirements due to the high number of interactions inside the different regions and between them. The most common computational approaches resort to the use of quantum mechanics (QM) to treat the active region through orbital- or density-based theories, and classical molecular mechanics (MM) for the environment. Originally proposed by Warshel and Levitt [1] in 1976, such combination of a QM description with a classical one has gained increasing popularity through the years [2-7] and represents now a standard approach to describe molecular systems of high complexity.

Within quantum-in-classical methods, different formulations can be adopted to describe the classical component either preserving its discrete molecular structure [8, 9] or through a coarser treatment as a continuum medium [10]. If we focus on the more realistic discrete methods, the simplest way to simulate the environmental effects is to assign partial atomic charges to the classical atoms, which create a perturbation on the active subsystem through a static external electric field. While its most common use is for ground-state problems, this approach has also been widely employed to compute excitation properties of (bio)molecules in complex environments with a varying degree of success. Several recent studies of the absorption properties of small organic molecules in solutions as well as photo-active proteins [11-25] have in fact provided substantial evidence that the choice of a static point-charges embedding scheme may qualitatively reproduce some experimental findings but appears inadequate if one strives for higher accuracy.

To cope with these limitations and simulate a more realistic interaction between the environment and the photo-active site, a more accurate embedding scheme is required which takes into account mutual polarization effects both in the ground state and in response to the excitation of the embedded quantum region (as long as a partitioning scheme with fixed number of electrons is applicable to the problem of interest). This can be achieved by describing the classical atoms not only with partial atomic charges but also with induced dipoles determined by the atomic polarizabilities and the total electric field of the system with contributions from the embedded electronic density, the static electric field of the point charges and the other induced dipoles in the environment. In this scheme, the QM subsystem is polarized by an MM region which can in turn respond to the presence of the embedded molecule in its ground or excited state [26-29]. Such a polarizable embedding (QM/MMpol) scheme has been combined with coupled cluster theory (CC) $[23,26,30,31]$, the complete-active-space self-consistent field (CASSCF) method [32], and time-dependent density functional theory (TDDFT) [27, 28, 33,34] and has provided a very effective description of excitation energies beyond static 
multipole models [23,34-37], allowing the systematic study of different polarization effects induced in the ground state and in response to the excitation of the solute $[15,25]$.

Here, we combine for the first time quantum Monte Carlo (QMC) methods with the reaction field of polarizable dipoles and investigate the performance of QMC/MMpol for the computation of the excitation energies of small solvated molecules, namely, methylenecyclopropene and acrolein in water. The use of QMC to compute electronic excitations has already been extensively validated in the gas phase [21,38-41] and also employed in combination with standard MM methods [17, 21, 25] and DFT embedding [42]. To explore the robustness of the QMC/MMpol scheme, we compare the excitation energies obtained in the presence of static and polarizable environments with the results of supermolecular calculations, where both solute and solvent are treated at the QM level. Alongside QMC, we also analyse the response to the different embedding conditions of other quantum mechanical methods for the treatment of the solute, namely, time-dependent density functional theory and the complete-active space second-order perturbation theory (CASPT2).

The paper is organized as follows. In Sec. 5.2, we discuss the theoretical background and the methodological aspects of the computation of the QMC/MMpol excitations and, in Sec. 5.3, give the computational details. In Sec. 5.4, we present the complete analysis of our results for methylenecyclopropene and acrolein, and conclude in Sec. 5.5.

\subsection{Methods}

In the QM/MMpol approach presented here, the solute and the solvent are treated at different levels of the theory, namely the solute is described quantum mechanically while the solvent is represented by explicit molecules treated with a classical model which consists of permanent atomic partial charges and atomic polarizabilities. The polarizabilities are used to determine the induced dipoles on the atoms of the solvent molecules.

Thus, the Schrödinger equation for the solute wave function becomes

$$
\left(\mathcal{H}_{\text {vac }}+\hat{\mathcal{V}}\right) \Psi=\mathrm{E} \Psi
$$

where the vacuum Hamiltonian describes the isolated solute and the second term the interaction between the solute and the classical solvent. Since the MM model has both static and polarization contributions, we have

$$
\hat{\mathcal{V}}=\sum_{\mathrm{m}}^{\mathrm{N}_{\mathrm{ch}}} q_{\mathrm{m}} \hat{V}_{\text {solute }}\left(\mathbf{r}_{\mathrm{m}}\right)-\sum_{\mathrm{a}}^{\mathrm{N}_{\text {dip }}} \boldsymbol{\mu}_{\mathrm{a}} \cdot \hat{\mathbf{E}}_{\text {solute }}\left(\mathbf{r}_{\mathrm{a}}\right),
$$

where $\hat{V}_{\text {solute }}\left(\mathbf{r}_{\mathrm{m}}\right)$ and $\hat{\mathbf{E}}_{\text {solute }}\left(\mathbf{r}_{\mathrm{a}}\right)$ are the electrostatic potential and electric field operators generated by the electrons and the nuclei at the coordinate $\mathbf{r}_{\mathrm{m}}$ of the point charge $q_{\mathrm{m}}$ and $\mathbf{r}_{\mathrm{a}}$ of the dipole $\boldsymbol{\mu}_{\mathrm{a}}$, respectively. 
The solvent contribution to the energy is given by

$$
\begin{aligned}
\mathrm{U}_{\text {solvent }} & =\frac{1}{2} \sum_{\mathrm{n}}^{\mathrm{N}_{\mathrm{ch}}} \sum_{\mathrm{m} \neq \mathrm{n}}^{\mathrm{N}_{\mathrm{ch}}} \frac{q_{\mathrm{m}} q_{\mathrm{n}}}{\left|\mathbf{r}_{\mathrm{m}}-\mathbf{r}_{\mathrm{n}}\right|}-\sum_{a}^{\mathrm{N}_{\text {dip }}} \boldsymbol{\mu}_{\mathrm{a}} \cdot \mathbf{E}_{q}\left(\mathbf{r}_{\mathrm{a}}\right) \\
& +\frac{1}{2} \sum_{\mathrm{a}}^{\mathrm{N}_{\text {dip }}} \sum_{\mathrm{b} \neq \mathrm{a}}^{\mathrm{N}_{\text {dip }}} \boldsymbol{\mu}_{\mathrm{a}} \cdot \mathbf{T}_{\mathrm{ab}} \cdot \boldsymbol{\mu}_{\mathrm{b}}+\frac{1}{2} \sum_{\mathrm{a}}^{\mathrm{N}_{\text {dip }}} \frac{\mu_{\mathrm{a}}^{2}}{\alpha_{\mathrm{a}}}
\end{aligned}
$$

where $\alpha_{\mathrm{a}}$ is the isotropic polarizability at site a, $\mathbf{E}_{q}\left(\mathbf{r}_{\mathrm{a}}\right)$ the static electric field generated by the point charges of the solvent at the sites of the dipoles,

$$
\mathbf{E}_{q}\left(\mathbf{r}_{\mathrm{a}}\right)=\sum_{\mathrm{b} \neq \mathrm{a}}^{\mathrm{N}_{\mathrm{ch}}} f_{1}\left(r_{\mathrm{ab}}\right) \frac{q_{\mathrm{m}}\left(\mathbf{r}_{\mathrm{a}}-\mathbf{r}_{\mathrm{b}}\right)}{\left|\mathbf{r}_{\mathrm{a}}-\mathbf{r}_{\mathrm{b}}\right|^{3}}
$$

and the dipole field tensor $\mathbf{T}_{\mathrm{ab}}$ is defined as

$$
\left(\mathbf{T}_{\mathrm{ab}}\right)_{i j}=\frac{f_{1}\left(r_{\mathrm{ab}}\right)}{r_{\mathrm{ab}}^{3}}\left[\delta_{i j}-3 f_{2}\left(r_{\mathrm{ab}}\right) \frac{\left(\mathbf{r}_{\mathrm{ab}}\right)_{i}\left(\mathbf{r}_{\mathrm{ab}}\right)_{j}}{r_{\mathrm{ab}}^{2}}\right],
$$

with $\mathbf{r}_{\mathrm{ab}}=\mathbf{r}_{\mathrm{a}}-\mathbf{r}_{\mathrm{b}}$ and $r_{\mathrm{ab}}=\left|\mathbf{r}_{\mathrm{ab}}\right|$. The first three terms in the solvent energy are the charge-charge, dipole-charge, and dipole-dipole interaction energies while the last term is the self-energy cost to induce the dipoles. In the last two equations, we have introduced two screening functions following the Thole linear model [43-45] to prevent divergences in the energy when two MM sites are too close. The two functions are defined as

$$
\begin{aligned}
& f_{1}\left(r_{\mathrm{ab}}\right)=4\left(\frac{r_{\mathrm{ab}}}{c}\right)^{3}-3 f_{2}\left(r_{\mathrm{ab}}\right) \\
& f_{2}\left(r_{\mathrm{ab}}\right)=\left(\frac{r_{\mathrm{ab}}}{c}\right)^{4},
\end{aligned}
$$

for $r_{\mathrm{ab}} / c \leq 1$ with $c=k\left(\alpha_{\mathrm{a}} \alpha_{\mathrm{b}}\right)^{1 / 6}$, and are identical to one otherwise. The dimensionless parameter $k$ is optimized together with the atomic polarizabilities in Ref. [46] to reproduce the experimental molecular polarizabilities of an extensive set of compounds. In this work, we consider water as solvent and interactions between charges and dipoles belonging to the same water molecule are excluded.

For a given solute wave function, $\Psi$, the energy in the presence of this polarizable solvent model is given by

$$
\mathrm{E}[\Psi, \boldsymbol{\mu}]=\left\langle\Psi\left|\mathcal{H}_{\mathrm{vac}}+\hat{\mathcal{V}}\right| \Psi\right\rangle+\mathrm{U}_{\text {solvent }},
$$

where the formalism so far has not assumed that the solute and the induced dipoles are in equilibrium, so wave function and dipoles are treated as independent entries in the energy expression. For a given wave function, the induced dipoles in equilibrium with the solute can then be obtained as the dipoles that minimize the energy $\mathrm{E}[\Psi, \boldsymbol{\mu}]$. 
This minimization leads to the expected relation between the induced dipoles and the total electric field:

$$
\boldsymbol{\mu}_{\mathrm{a}}=\alpha_{\mathrm{a}}\left[\mathbf{E}_{\text {static }}\left(\mathbf{r}_{\mathrm{a}}\right)-\sum_{\mathrm{b} \neq \mathrm{a}}^{\mathrm{N}_{\mathrm{dip}}} \mathbf{T}_{\mathrm{ab}} \cdot \boldsymbol{\mu}_{\mathrm{b}}\right],
$$

where the static field due to the solute charge density and the solvent permanent charge distribution is given by

$$
\mathbf{E}_{\text {static }}\left(\mathbf{r}_{\mathrm{a}}\right)=\left\langle\Psi\left|\hat{\mathbf{E}}_{\text {solute }}\left(\mathbf{r}_{\mathrm{a}}\right)\right| \Psi\right\rangle+\mathbf{E}_{q}\left(\mathbf{r}_{\mathrm{a}}\right),
$$

with

$$
\left\langle\Psi\left|\hat{\mathbf{E}}_{\text {solute }}\left(\mathbf{r}_{\mathrm{a}}\right)\right| \Psi\right\rangle=\int \rho_{\text {solute }}(\mathbf{r}) \frac{\left(\mathbf{r}_{\mathrm{a}}-\mathbf{r}\right)}{\left|\mathbf{r}-\mathbf{r}_{\mathrm{a}}\right|^{3}} \mathrm{~d} \mathbf{r}
$$

and $\rho_{\text {solute }}$ the solute nuclear and electronic charge density [47]. This set of equations defines the optimal dipoles and can be rewritten as

$$
\mathbf{A} \boldsymbol{\mu}=\mathbf{E}_{\text {static }}
$$

where $\boldsymbol{\mu}$ and $\mathbf{E}_{\text {static }}$ are vectors of dimension $3 \mathrm{~N}_{\text {dip }}$, which contain all the dipoles and the static electric field at the corresponding positions, respectively, while the $3 \mathrm{~N}_{\text {dip }} \times 3 \mathrm{~N}_{\text {dip }}$ matrix $\mathbf{A}$ only depends on the values of $\alpha_{a}$ and the positions of the solvent molecules.

Importantly, since the dipoles $\boldsymbol{\mu}$ and the wave function $\Psi$ are mutually dependent (Eqs. 5.8 and 5.9), they must be brought to self consistency, a procedure which results in a different sets of induced dipoles for the ground and the excited states. In other words, the Schrödinger equation (Eq. 5.1) coupling the solute to classical polarizable dipoles is non-linear being characterized by a potential $\hat{\mathcal{V}}$ which depends on the wave function quadratically through the induced dipoles (Eq. 5.8). Solving this equation leads to a set of non-orthogonal wave functions in equilibrium with their corresponding dipoles. When wave function and dipoles are in equilibrium, the energy of the solute and the solvent (Eq. 5.7) becomes

$$
\begin{aligned}
\mathrm{E}[\Psi, \boldsymbol{\mu}(\Psi)] & =\left\langle\Psi\left|\mathcal{H}_{\text {vac }}+\sum_{\mathrm{m}}^{\mathrm{N}_{\mathrm{ch}}} q_{\mathrm{m}} \hat{V}_{\text {solute }}\left(\mathbf{r}_{\mathrm{m}}\right)\right| \Psi\right\rangle \\
& -\frac{1}{2} \sum_{\mathrm{a}}^{\mathrm{N}_{\text {dip }}} \boldsymbol{\mu}_{\mathrm{a}}(\Psi) \cdot\left[\left\langle\Psi\left|\hat{\mathbf{E}}_{\text {solute }}\left(\mathbf{r}_{\mathrm{a}}\right)\right| \Psi\right\rangle+\mathbf{E}_{q}\left(\mathbf{r}_{\mathrm{a}}\right)\right] \\
& +\frac{1}{2} \sum_{\mathrm{n}}^{\mathrm{N}_{\mathrm{ch}}} \sum_{\mathrm{m} \neq \mathrm{n}}^{\mathrm{N}_{\mathrm{ch}}} \frac{q_{\mathrm{m}} q_{\mathrm{n}}}{\left|\mathbf{r}_{\mathrm{m}}-\mathbf{r}_{\mathrm{n}}\right|}
\end{aligned}
$$

where the dipoles $\boldsymbol{\mu}(\Psi)$ are determined through Eq. 5.8. It is important to stress that this simplified expression for the energy does not hold when the dipoles and the electric field of the solute are not in equilibrium. This occurs for instance when 
one computes an excitation energy in the presence of dipoles which are in equilibrium with one of the two states of interest: One solves the Schrödinger equation for state $I$ to determine $\Psi_{I}$ and $\boldsymbol{\mu}\left(\Psi_{I}\right)$ and computes the other state $J$ in the potential $\mathcal{V}$ of the fixed dipoles $\mu\left(\Psi_{I}\right)$ to obtain the excitation energy as $\Delta \mathrm{E}=$ $\mathrm{E}\left[\Psi_{J}, \boldsymbol{\mu}\left(\Psi_{I}\right)\right]-\mathrm{E}\left[\Psi_{I}, \boldsymbol{\mu}\left(\Psi_{I}\right)\right]$, where the solvent contribution $\mathrm{U}_{\text {solvent }}$ cancels out in this energy difference since the dipoles are the same for both states. Another non-equilibrium situation is encountered when we optimize a wave function in the presence of dipoles which are optimal for the initial starting wave function. Then, we must employ the general expressions for the coupling of the solute wave function to a set of non-equilibrium induced dipoles (Eqs. 5.2, 5.3, and 5.7).

In this work, we focus on the computation of the excitation energies of a solvated molecule in such a classical polarizable model using QMC to describe the solute, and consider two different polarization regimes. In the first case, the induced dipoles are in equilibrium with the solute ground-state density and used in the computation of the ground and excited states (polGS). In the second case, state-specific dipoles are generated with respect to the ground- and the excited-state static electric field separately (polSS). To this aim, two parallel routes (I and II) are followed to obtain the polGS and polSS excitation energies according to the steps outlined below, where we denote with the subscripts 0 and 1 the ground and the excited state and with the superscripts "g" and "e" whether the wave functions are computed in the potential of the induced dipoles $\boldsymbol{\mu}^{g}$ and $\boldsymbol{\mu}^{e}$ polarized to the ground- and the excited-state solute density, respectively. Starting from iteration $i=1$, we have

Ia) Determine $\boldsymbol{\mu}^{g}(i-1)$ in equilibrium with $\Psi_{0}^{g}(i-1)$.

Ib) Optimize $\Psi_{0}^{g}(i)$ and $\Psi_{1}^{g}(i)$ in the presence of the ground-state dipoles $\boldsymbol{\mu}^{g}(i-1)$.

Ic) Return to Ia.

At self-consistency, we compute the polGS excitation energy as

$$
\Delta \mathrm{E}_{\mathrm{polGS}}=\mathrm{E}\left[\Psi_{1}^{\mathrm{g}}, \boldsymbol{\mu}^{\mathrm{g}}\right]-\mathrm{E}\left[\Psi_{0}^{\mathrm{g}}, \boldsymbol{\mu}^{\mathrm{g}}\right] \text {. }
$$

For the polSS calculation,

IIa) Determine $\boldsymbol{\mu}^{\mathrm{e}}(i-1)$ in equilibrium with $\Psi_{1}^{e}(i-1)$.

IIb) Optimize $\Psi_{0}^{\mathrm{e}}(i)$ and $\Psi_{1}^{\mathrm{e}}(i)$ in the presence of the excited-state dipoles $\boldsymbol{\mu}^{\mathrm{e}}(i-1)$.

IIc) Return to IIa.

The polSS excitation energy is then obtained as

$$
\Delta \mathrm{E}_{\mathrm{polSS}}=\mathrm{E}\left[\Psi_{1}^{\mathrm{e}}, \boldsymbol{\mu}^{\mathrm{e}}\right]-\mathrm{E}\left[\Psi_{0}^{\mathrm{g}}, \boldsymbol{\mu}^{\mathrm{g}}\right]
$$

The starting wave functions $\Psi_{0}(0)$ and $\Psi_{1}(0)$ are obtained in the presence of the static classical point charges and, at each iteration, the wave functions are optimized in a state-average (SA) fashion since the systems considered here have no symmetry. 


\begin{tabular}{cccc}
\hline Method & iter. no. & \multicolumn{2}{c}{$\mathrm{E}_{\mathrm{exc}}(\mathrm{eV})$} \\
\cline { 3 - 4 } & & $\mathrm{VMC}$ & $\mathrm{DMC}$ \\
\hline QM/polGS & 1 & $5.138(3)$ & $5.120(7)$ \\
& 2 & $5.134(3)$ & $5.119(7)$ \\
& 3 & $5.128(3)$ & $5.110(7)$ \\
QM/polSS & 1 & $5.010(3)$ & $4.999(7)$ \\
& 2 & $5.012(3)$ & $5.006(7)$ \\
& 3 & $5.015(3)$ & $5.009(7)$ \\
\hline
\end{tabular}

Table 5.1: Convergence of the polGS and polSS excitation energies (eV) obtained in VMC and DMC with the number of wave function-dipole optimization cycles for the $\pi \rightarrow \pi^{*}$ state of methylenecyclopropene in water. The statistical error on the QMC values is given in brackets.

We note that, while in the polGS excitation energy the solvent energy $\mathrm{U}_{\text {solvent }}$ cancels out as already mentioned above, this is not the case in the polSS energy difference since two different sets of dipoles are employed.

To describe the quantum solute, we use both the variational (VMC) and diffusion Monte Carlo (DMC) methods. We employ many-body molecular wave functions of the Jastrow-Slater form,

$$
\Psi=\mathcal{J} \sum_{i=1}^{\mathrm{N}_{\mathrm{CSF}}} c_{i} C_{i},
$$

where $c_{i}$ are the expansion coefficients of the configuration state functions (CSFs), $C_{i}$, and $\mathcal{J}$ is the Jastrow correlation factor which has an explicit dependence on the inter-particle distances. In VMC, the energy of the electronic states is computed according to Eq. 5.7 where the expectation values are calculated via Monte Carlo sampling of the square of the wavefunction, $\Psi^{2}$. For the given wave function, the average electric field due to the solute sampled at the dipole sites is then used to compute the induced dipoles (Eq. 5.8). The Jastrow-Slater wave functions are fully optimized by energy minimization in VMC [48] in a state-average fashion [38], using $\sum_{I}\left|\Psi_{I}\right|^{2}$ as square of the guiding wave function.

After we have followed schemes I and II above to obtain the self-consistent wave functions and dipoles in VMC, we perform DMC calculations to compute the polGS and polSS excitations energies, where we employ $\Psi_{0}^{g}$ and $\Psi_{1}^{g}$ as trial wave functions in the potential generated by $\boldsymbol{\mu}^{\mathrm{g}}$, and $\Psi_{1}^{e}$ in combination with $\boldsymbol{\mu}^{\mathrm{e}}$. For the molecules considered here, the VMC and DMC excitation energies are converged after two wave function-dipole optimization cycles as reported for the methylenecyclopropene molecule in polarizable water solvent in Table 5.1.

\subsection{Computational details}

The QMC calculations are carried out with the program package CHAMP [49]. We employ scalar-relativistic energy-consistent Hartree-Fock pseudopotentials [50,51] 
and obtain the starting determinantal component of the Jastrow-Slater wave function in complete-active-space self-consistent field (CASSCF) calculations performed with the program GAMESS(US) [52,53]. For acrolein, we truncate the CAS expansion expressed on the state-average natural orbitals with an appropriate threshold of 0.03 on the coefficients of the CSFs (the total CASSCF weight of all states is greater than 98.5\%), and retain the union set of surviving CSFs for the states of interest. We use instead the complete CAS space for methylenecyclopropene (MCP). We employ a two-body Jastrow factor $\left(\mathcal{J}_{2 \text {-body }}\right)$ to account for electron-nuclear and electron-electron correlations and use different Jastrow factors to describe different atom types [54]. The effect of using a three-body Jastrow factor $\left(\mathcal{J}_{3-\text { body }}\right)$ including electron-electron-nucleus correlation is also tested. The QMC wave functions are fully optimized within VMC using the linear method [48] and its extension to stateaverage calculations [38], and used to compute excitation energies at both the VMC and the DMC level. We treat the pseudopotentials beyond the locality approximation [55] and use an imaginary time step of 0.075 a.u. in the DMC supermolecular calculations and of 0.05 a.u. in all other DMC runs. In order to prevent divergences of the electrostatic interactions at the coalescence points, we introduce a small cutoff distance of 0.04 a.u. and, when an electron is closer than this distance to the site of a point charge or a dipole, we set the interaction equal to the value it takes at the cutoff. For historical reasons, the QMC calculations with point charges only employ charges which are screened according to the function $v_{q}(r)=q\left[1-\exp \left(-B r^{2}\right)\right] / r$.

We use the MOLCAS 7.4 suite of programs [56] for the CASPT2 [57, 58] calculations and always employ the recommended zero-order Hamiltonian [59] with the IPEA shift set to 0.25 a.u. We use an additional constant imaginary shift [60] of 0.1 a.u. and, unless otherwise noted, we report the excitation energies computed at the single-state level. For direct comparison with the QMC results, we employ pseudopotentials $[50,51]$ also in the MOLCAS calculations. The CASPT2/MMpol results are computed in a two-step procedure recently presented [32], where the MM induced dipoles and charges from an all-electron CASSCF/MMpol calculation with the Gaussian code [61] are later used in MOLCAS as a static external potential to obtain the CASPT2 results. Such CASSCF/MMpol calculations are performed using a locally modified version of Gaussian09, revision A.02, and are obtained using the state-average procedure for the ground and excited states and adapting the MM polarization either to the ground state (polGS), or to the ground and excited states in two separate calculations (polSS). All-electron CASPT2 calculations are reported in the SI, where we freeze as many $\sigma$ orbitals as there are heavy atoms.

We perform the TDDFT calculations using a locally modified version of Gaussian09, revision A.02 [61] and the CAM-B3LYP [62] exchange-correlation functional. The range-separated hybrid functional CAM-B3LYP is chosen to limit possible spurious charge-tranfer effects in the supermolecular calculations.

In the QM/MMpol calculations, the water molecules are described by point charges derived from a standard restrained electrostatic potential (RESP) fit using the electrostatic potential computed at the MP2/aug-cc-pVTZ level on the TIP3P water geometry ( $\mathrm{q}_{\mathrm{O}}=-0.726, \mathrm{q}_{\mathrm{H}}=0.363$ a.u.) [63] and by isotropic atomic polarizabilities taken from the Amber pol12 force field (AL model in Ref. [46, 64]). The parameter 

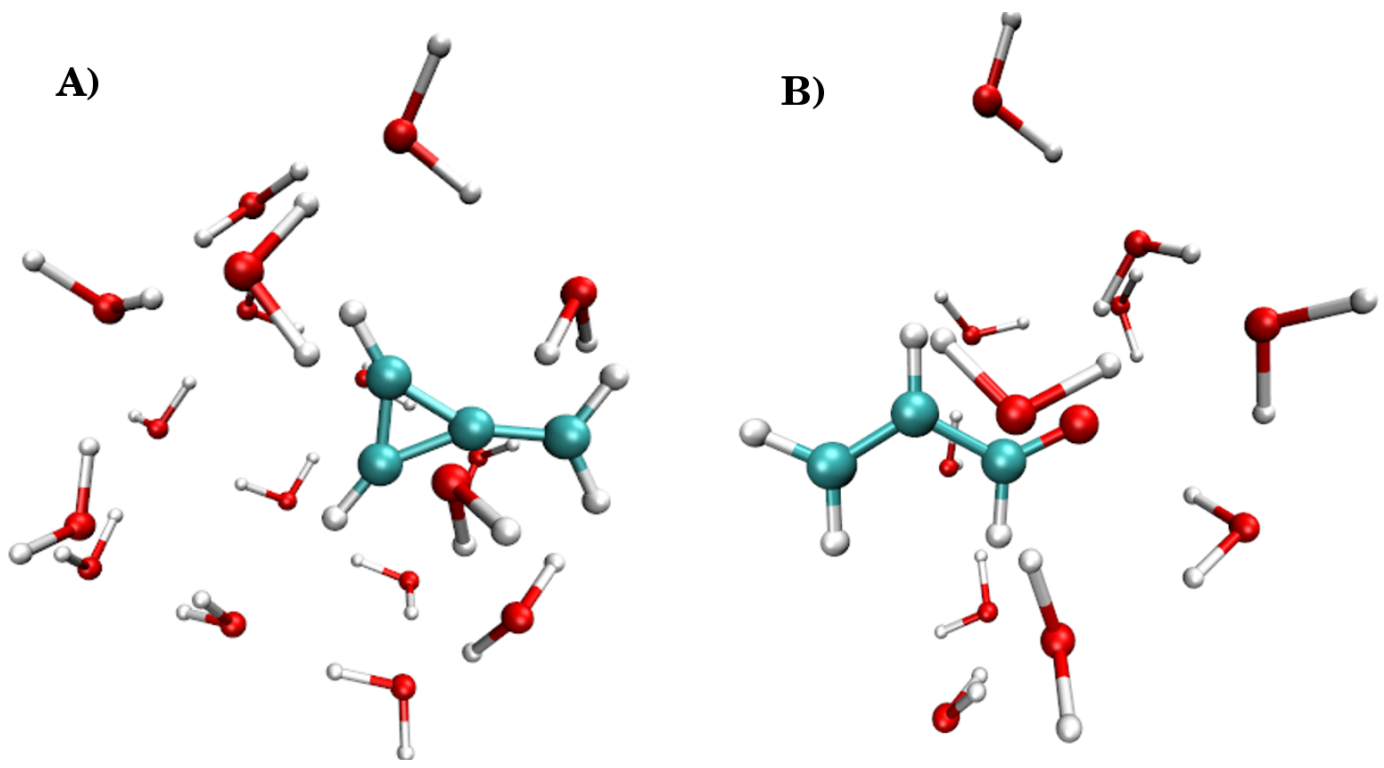

Figure 5.1: Cluster models of solvated (A) methylenecyclopropene and (B) $s$-trans acrolein in water.

$k$ (Eq. 5.6) is set to 2.5874 (AL model in Ref. [46]). We also use the non-polarizable TIP3P model ( $\mathrm{q}_{\mathrm{O}}=-0.834$ and $\mathrm{q}_{\mathrm{H}}=0.417$ a.u.). [65] The screening parameters in the QMC calculations with point charges are $B_{\mathrm{O}}=0.52548$ and $B_{\mathrm{H}}=2.73465$ a.u.

\subsubsection{Cluster models}

In this study, we use small water clusters of solvated methylenecyclopropene (MCP) and $s$-trans acrolein. This allows us to perform supermolecular reference calculations of the excitation energies which can be used to assess the quality of the results obtained with different embedding approaches.

We generate the cluster models by solvating MCP and acrolein in approximately one shell of water molecules (17 and 19 molecules, respectively) and optimize the geometries with Gaussian09 in DFT with the BLYP exchange-correlation functional [66, 67] and the cc-pVDZ basis set. For acrolein, due to the high number of tests performed to ascertain the supermolecular value of the excitation energy, we reduce the size of the cluster and retain the 11 water molecules closer to the carbonyl oxygen of acrolein. The final cluster models used in this study are shown in Figure 5.1.

\subsubsection{Choice of basis set and Jastrow factor in QMC}

In the QMC calculations, we employ the Gaussian basis sets [50,68] specifically constructed for our pseudopotentials. In Table 5.2, we assess the basis-set convergence of the QMC excitation energies of MCP isolated and in TIP3P water using the cc-pVTZ', aug-cc-pVDZ', and aug-cc-pVTZ' basis sets, where the prime indicates that cc-pVDZ is employed for hydrogen. We find that the use of augmentation 
significantly accelerates the convergence of the $\mathrm{VMC}$ and DMC excitation energies and that the aug-cc-pVDZ' basis set yields results at the DMC level that are only 0.02-0.03 eV higher than those obtained with the larger aug-cc-pVTZ'. In Table 5.2, we also investigate the effect of including three-body terms in the Jastrow factor and find that it generally leads to higher excitation energies, the shift being larger in the isolated system and in combination with the aug-cc-pVDZ' basis set. The error with respect to the triple- $\zeta$ result is about $0.05 \mathrm{eV}$ at the $\mathrm{VMC}$ level and the basis-set convergence of the excitation energy therefore appears to be slower in the presence of a three-body Jastrow factor. Consequently, all QMC results presented below are obtained with the aug-cc-pVDZ' basis set and the two-body Jastrow factor, which offer a good compromise between accuracy and computational cost.

\begin{tabular}{lcccccc}
\hline & pVTZ' & \multicolumn{2}{c}{ aug-cc-pVDZ } & & \multicolumn{2}{c}{ aug-cc-pVTZ' } \\
\cline { 7 - 7 } & $\mathcal{J}_{2 \text {-body }}$ & $\mathcal{J}_{2 \text {-body }}$ & $\mathcal{J}_{3 \text {-body }}$ & & $\mathcal{J}_{2 \text {-body }}$ & $\mathcal{J}_{3 \text {-body }}$ \\
\hline VMC/isolated & $4.265(1)$ & $4.179(1)$ & $4.225(1)$ & & $4.161(1)$ & $4.170(1)$ \\
DMC/isolated & $4.229(1)$ & $4.159(1)$ & $4.185(1)$ & & $4.141(1)$ & $4.151(1)$ \\
VMC/TIP3P & $5.010(0)$ & $4.889(1)$ & $4.908(1)$ & & $4.857(0)$ & $4.862(1)$ \\
DMC/TIP3P & $4.963(1)$ & $4.862(1)$ & $4.867(1)$ & & $4.831(1)$ & $4.829(1)$ \\
\hline
\end{tabular}

Table 5.2: Basis-set convergence of the VMC and DMC excitation energies (eV) of the $\pi \rightarrow \pi^{*}$ state of MCP isolated and in TIP3P water. Either a two- or a threebody Jastrow factor is employed. The statistical error on the QMC values is given in brackets.

In the QMC supermolecular calculations, we employ the aug-cc-pVDZ' basis set for the solute and the closest water molecules and describe the remaining solvent molecules with the cheaper cc-pVDZ on the oxygens and cc-VDZ basis set (without $p$ functions) on the hydrogens. In the MCP and acrolein clusters, we identify eight and five close water molecules, respectively, so that the use of different basis sets for the rest of the solvent affects the CASSCF excitation energies to less than $0.01 \mathrm{eV}$. In the wave function optimization, we localize the orbitals and do not to optimize those of the far water molecules to limit the computational cost.

\subsubsection{Basis-set convergence in CASPT2 and TDDFT}

We test the basis set convergence of the CASPT2 and TDDFT excitation energies for MCP using the Dunning's correlation consistent cc-pVXZ and aug-cc-pVXZ series up to quintuple and quadruple $\zeta$, respectively [69-72]. We find that for MCP isolated and in water, the inclusion of augmentation is very important (more so than in the QMC calculations) to accelerate the basis set convergence, and the excitation energies computed with the aug-cc-pVDZ basis are compatible with the aug-cc-pVQZ and cc-pV5Z values within $0.02-0.03 \mathrm{eV}$ at both level of theory.

Therefore, we select the aug-cc-pVDZ as default basis set for the computation of the TDDFT excitation energies. For CASPT2, we compare the excitation energies computed with pseudopotentials in combination with the aug-cc-pVDZ' basis 
set described above to the all-electron values obtained with the Dunning's aug-ccpVDZ basis. As reported in Table 5.3, the resulting excitation energies are compatible within $0.04 \mathrm{eV}$ for MCP and the $\mathrm{n} \rightarrow \pi^{*}$ excitation of acrolein, while the difference is of the order of $0.1 \mathrm{eV}$ for the $\pi \rightarrow \pi^{*}$ excitation energy of acrolein. Surprisingly, in all three cases, the CASSCF values computed with and without pseudopotential agree to better than $0.02 \mathrm{eV}$. To allow a direct comparison with the QMC results, we report below the CASPT2 values obtained with pseudopotentials and the aug-cc-pVDZ' basis set.

\begin{tabular}{lcccccc}
\hline MCP & isolated & TIP3P & nopol & polGS & polSS & super \\
\hline CASSCF $\pi \rightarrow \pi^{*}$ & & & & & & \\
all-electron & 4.211 & 4.963 & 4.864 & 5.103 & 4.997 & 5.062 \\
pseudopotentials & 4.217 & 4.965 & 4.866 & 5.104 & 4.989 & 5.046 \\
CASPT2 $\pi \rightarrow \pi^{*}$ & & & & & & \\
all-electron & 4.111 & 4.876 & 4.776 & 5.019 & 4.921 & 4.968 \\
pseudopotentials & 4.133 & 4.902 & 4.801 & 5.045 & 4.928 & 4.978 \\
\hline$s$-trans acrolein & & & & & & \\
\hline CASSCF n $\rightarrow \pi^{*}$ & & & & & & \\
all-electron & 3.726 & 4.486 & 4.386 & 4.684 & 4.588 & 4.533 \\
pseudopotentials & 3.701 & 4.465 & 4.365 & 4.663 & 4.560 & 4.534 \\
CASPT2 n $\rightarrow \pi^{*}$ & & & & & & \\
all-electron & 3.565 & 4.256 & 4.167 & 4.434 & 4.355 & 4.237 \\
pseudopotentials & 3.559 & 4.213 & 4.127 & 4.385 & 4.297 & 4.224 \\
CASSCF $\pi \rightarrow \pi^{*}$ & & & & & & \\
all-electron & 7.799 & 7.064 & 7.118 & 7.007 & 6.960 & 7.010 \\
pseudopotentials & 7.846 & 7.111 & 7.164 & 7.047 & 7.002 & 7.031 \\
CASPT2 $\pi \rightarrow \pi^{*}$ & & & & & & \\
all-electron & 6.640 & 6.341 & 6.395 & 6.284 & 6.251 & 6.134 \\
pseudopotentials & 6.727 & 6.431 & 6.494 & 6.366 & 6.337 & 6.221 \\
\hline
\end{tabular}

Table 5.3: CASSCF and single-state CASPT2 excitation energies (eV) of methylenecyclopropene (MCP) and $s$-trans acrolein computed with and without pseudopotentials. We use a CAS $(4,4)$ expansion for methylenecyclopropene and a CAS $(6,10)$ for acrolein. In the all-electron calculations, we use the Dunning's augcc-pVDZ basis set and, in the pseudopotential calculations, the cc-pVDZ basis set specifically constructed for the pseudopotentals used in QMC and augmented on the heavy atoms (aug-cc-pVDZ' basis set) as detailed in the paper. In the all-electron CASPT 2 calculations, we freeze as many core $\sigma$ orbitals as there are heavy atoms. 


\subsection{Results}

We investigate here how the MMpol description of the environment affects the excitation energies computed with QMC for methylenecyclopropene and $s$-trans acrolein solvated in water. We treat the environment using two different MMpol schemes: In the polGS one, the environment is polarized only to the ground state and frozen in the computation of the excitation energy while, in the polSS approach, we include the response of the solvent to the excitation of the solute, equilibrating the dipoles with the ground- and excited-state density separately for each state. As elaborated in the Method Section, the resulting state-specific dipoles are then employed to compute the polSS excitation energy and to account in this manner for differential polarization effects. The polGS and polSS treatments of the environment capture the purely electrostatic interaction of the solute with the solvent, so possible discrepancies with the reference can be attributed not only to the approximate nature of the model but also to the incomplete description of solute-solvent coupling.

To understand the interplay between these environmental effects, the limitation of the embedding model, and the choice of quantum method for the solute, we employ TDDFT and CASPT2 in addition to QMC to compute the polGS and polSS excitation energies. Furthermore, we obtain the TDDFT excitation energies also in the linear response regime (polLR) of MMpol, where the environment does relax upon excitation of the solute but the induced dipoles respond to the transition density instead of the density difference between the ground and the excited state. The polLR model has been shown to miss the electrostatic response of the environment described in the polSS approximation but to capture the solvent polarization oscillating at the frequency of the solute excitation [73]. Both terms should however be present to describe the full environmental response and the analysis of the difference in the polSS and polLR responses at the TDDFT level will also help us to unravel the performance of the MMpol model in combination with QMC and other wave function approaches. Finally, we also compute the excitation energies of the solute at all levels of theory in a non-polarizable solvent of only point charges. We consider two different set of charges, namely, the point charges of the TIP3P water model and those (denoted as "nopol") from the polarizable force field, which we also employ in the MMpol calculations but use in this case without the corresponding polarizabilities.

We focus here on the vertical excitation energies of the $\pi \rightarrow \pi^{*}$ state of MCP and the $\mathrm{n} \rightarrow \pi^{*}$ and $\pi \rightarrow \pi^{*}$ states of acrolein. From absorption experiments in different solvents, we infer that water solvent induces a red-shift in the $\pi \rightarrow \pi^{*}$ excitation energy of acrolein with respect to the isolated value and a blue-shift for the other two states $[74,75]$. Since we only consider one geometrical configuration for each system, we expect a qualitatively similar behavior of the excitation energies computed on our cluster models but a detailed comparison with absorption experiments in solution is outside the scope of this paper. 


\begin{tabular}{lccccccc}
\hline Method & isolated & TIP3P & nopol & polGS & polSS & polLR & super \\
\hline VMC & $4.179(1)$ & $4.889(1)$ & $4.800(3)$ & $5.134(3)$ & $5.012(3)$ & - & $5.07(2)$ \\
DMC & $4.159(1)$ & $4.862(1)$ & $4.773(2)$ & $5.119(7)$ & $5.006(7)$ & - & $5.04(2)$ \\
CASPT2 & 4.133 & 4.902 & 4.801 & 5.045 & 4.928 & - & 4.978 \\
TDDFT & 4.224 & 5.017 & 4.916 & 5.190 & 5.077 & 5.173 & 5.187 \\
\hline
\end{tabular}

Table 5.4: Excitation energies (eV) of the $\pi \rightarrow \pi^{*}$ state of MCP isolated and solvated in a water cluster. The statistical error on the QMC values is given in brackets.

\subsubsection{Methylenecyclopropene}

MCP is a cross-conjugated molecule with interesting electrostatic features in the ground and first $\pi \rightarrow \pi^{*}$ excited states. Although without polar bonds with heteroatoms, MCP displays a relatively large dipole moment in the ground state (with an experimental value of about -1.9 Debye $[74,76]$ ) being characterized by an accumulation of $\pi$-electron density on the exocyclic double bond. The $\pi \rightarrow \pi^{*}$ excited state exhibits strong charge transfer from this double bond to the molecular ring, and the direction of the molecular dipole moment is reversed in going from the ground to the excited state. Consequently, a polar solvent in equilibrium with the ground-state solute is expected to destabilize the $\pi \rightarrow \pi^{*}$ excited state and to induce a blue-shift of the vertical excitation with respect to the gas-phase value [77]. Given the dramatic change in electronic structure upon excitation, we anticipate a strong response of the excitation energy to changes in the description of the environment. In particular, accounting for differential polarization effects via state-specific embedding should lead to a significant improvement on the use of a frozen (non-polarizable or polGS) solvent.

Our findings on the MCP-water model described above are summarized in Table 5.4 and Figure 5.2. In all calculations, the $\pi \rightarrow \pi^{*}$ excited state is always the second root and, at the correlated level, we employ a reference minimal CAS expansion correlating four electrons in two bonding and two anti-bonding $\pi$ orbitals. In line with the physical picture given above, we find that all methods predict a large blue-shift in the excitation energy (about $0.7-0.8 \mathrm{eV}$ ) from isolated MCP to MCP solvated in TIP3P water. The use of different point charges extracted from the polarizable force field (nopol) results in a rather similar trend. Polarizing the dipoles to the ground state of the solute (polGS) induces an additional significant blue-shift since a frozen environment optimal in the ground state further destabilizes the excited state. The shift with respect to nopol is somewhat larger in QMC and of the order of 0.35 $\mathrm{eV}$. As expected, accounting for differential polarization effects via polSS reverses the trend, lowering the excitation energies with respect to the polGS values. Given the greatly different nature of the two states, the polSS correction to polGS is significant (about $0.1 \mathrm{eV}$ ) in all methods and somewhat overshoots the supermolecular values. Nevertheless, the use of state-specific embedding is crucial to reach an agreement as good as $0.05 \mathrm{eV}$ with the supermolecular excitation energies at the correlated level.

We find that QMC yields excitation energies in very good agreement with the 


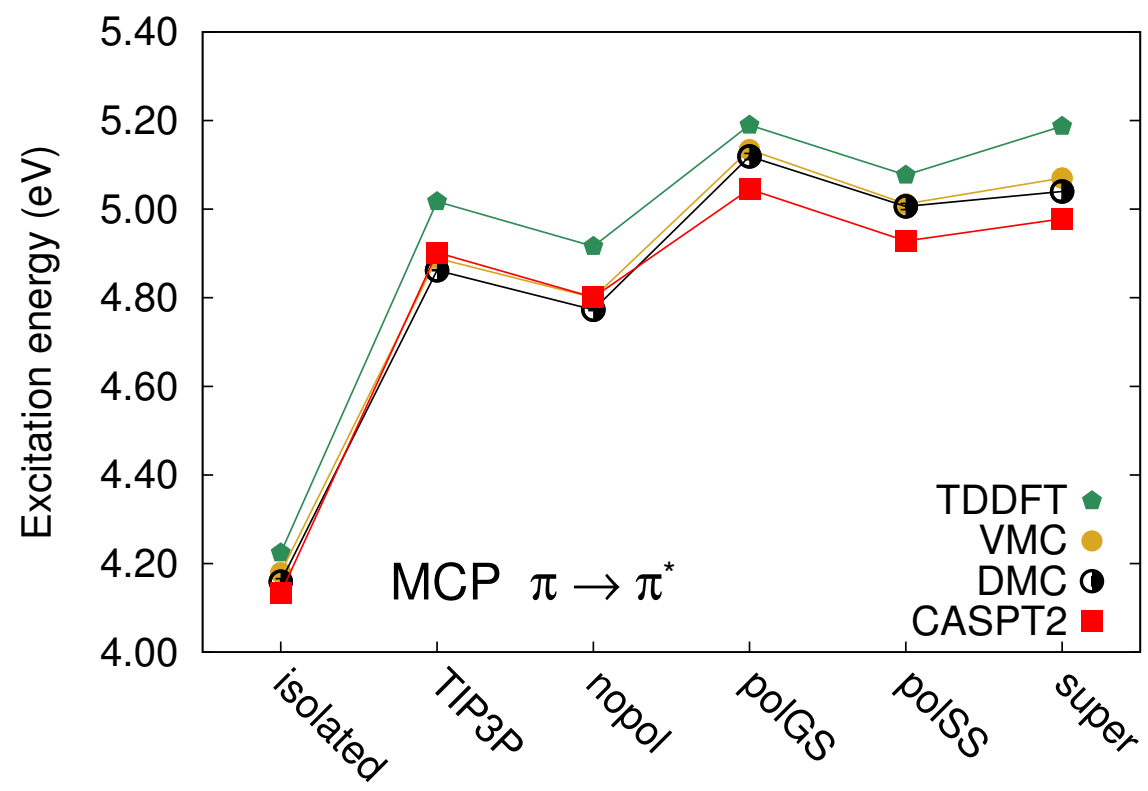

Figure 5.2: Excitation energies of the $\pi \rightarrow \pi^{*}$ state of MCP with different quantum methods and embedding schemes.

CASPT2 values in non-polarizable embedding while the two quantum methods predict a different shift from static point charges to polGS, and maintain hereinafter a similar behavior at the polSS and supermolecular levels. For TDDFT, the excitation energies computed with the CAM-B3LYP exchange-correlation functional respond less strongly to polGS than the QMC ones, with a shift from nopol somewhat closer to the CASPT2 value. Finally, we observe that the inclusion of the solvent relaxation in linear response (polLR) yields an excitation energy only slightly lower than the polGS value. The smaller effect with respect to a state-specific electrostatic treatment is to be expected since the response of the dipoles in the polLR model is proportional to the transition dipole moment which in the $\pi \rightarrow \pi^{*}$ transition of MCP is rather small.

\subsection{2 $s$-trans acrolein}

As in the case of MCP, the $\mathrm{n} \rightarrow \pi^{*}$ excited state of acrolein has charge-transfer character with the electronic density moving from the oxygen to the carbon skeleton upon excitation. This flux of charge is responsible for the reduction of the excited-state molecular dipole moment with respect to the ground state. Therefore, the vertical $n$ $\rightarrow \pi^{*}$ excitation energy blue-shifts in a polar environment with respect to the isolated molecule since the excited state is destabilized with respect to the ground state [78]. Again, we expect a significant difference between the excitation energies computed within a polarizable and a frozen environment and, in particular, the relaxation of the excited-state dipoles in the polSS embedding scheme should have a strong effect on 
the excitation. Since the $\mathrm{n} \rightarrow \pi^{*}$ transition is symmetry forbidden in the gas phase and retains a small transition dipole moment also in water, we do not expect polLR to considerably affect the excitation energies with respect to the polGS treatment.

We also consider the $\pi \rightarrow \pi^{*}$ singlet transition, which has rather a different nature than the $\mathrm{n} \rightarrow \pi^{*}$ one, being dipole allowed and associated with an increment of the molecular dipole moment. Therefore, the $\pi \rightarrow \pi^{*}$ transition is red-shifted in polar environments with respect to the isolated value [79]. In addition to a polSS correction on the polGS excitation energy due to the non-negligible change in molecular dipole moment between the ground and excited states, we expect that, when computing the excitations within TDDFT, polLR should recover sizable polarization effects considering the bright nature of the excited state and the correspondingly large transition dipole moment .

We describe the $\mathrm{n} \rightarrow \pi^{*}$ and $\pi \rightarrow \pi^{*}$ excitation energies of acrolein at the correlated level using a CAS $(6,10)$ expansion, which includes two bonding and six antibonding $\pi$ orbitals on the molecular chain, the $\sigma$ lone pair localized on the carbonyl oxygen and its anti-bonding $\sigma^{*}$ orbital. This large active space allows a balanced treatment of the two excited states at the same time in a SA-CASSCF calculation. In particular, the choice of the CAS $(6,10)$ reference active space is crucial to give a proper description of the $\pi \rightarrow \pi^{*}$ excited state, which shows a slow convergence with the size of the active space.

While the $\mathrm{n} \rightarrow \pi^{*}$ state is always the second root of the SA-CASSCF wavefunction, the bright $\pi \rightarrow \pi^{*}$ state in the isolated system is the fifth root and becomes the fourth state after the perturbation correction is introduced in CASPT2. The same behavior is observed in QMC after the inclusion of dynamic correlation through the Jastrow factor. The presence of a solvent simplifies the treatment of the $\pi \rightarrow \pi^{*}$ state, which becomes the third root at the SA-CASSCF level, and the perturbation and QMC calculations can then be performed using only three states. In the following, we present the CASPT2 excitation energies computed at the single-state level since, for the isolated system, we observe a strong mixing of the eigenvectors of the three highest roots in the multi-state CASPT2 calculations. The $\mathrm{n} \rightarrow \pi^{*}$ and $\pi \rightarrow \pi^{*}$ excitation energies of our solvated cluster are reported in Table 5.5 and Figure 5.3.

For the $\mathrm{n} \rightarrow \pi^{*}$ state, all quantum methods respond rather similarly to the presence of a non-polarizable environment, predicting a blue-shift of about $0.5-0.7 \mathrm{eV}$ depending on the set of point charges (TIP3P or nopol) considered. Improving only the ground-state description of the solvent with the polGS scheme consistently induces a further blue-shift of more than $0.2 \mathrm{eV}$ with respect to the nopol values, thus generally overshooting the value of the supermolecular reference. The inclusion of differential polarization effects corrects the polGS results, albeit to a lesser extent than in MCP, bringing the excitation energies in closer agreement with the reference. As expected, the polLR correction is negligible for this state. We must note that a TIP3P description appears here to outperform polSS within TDDFT and CASPT2, which is possibly a fortuitous coincidence due to the use of a single, highly asymmetric configuration with a limited number of water molecules to describe the solution. Finally, it is interesting to observe that the supermolecular excitation energies have a considerable spread, with the TDDFT value being much smaller than the CASPT2 


\begin{tabular}{lccccccc}
\hline & isolated & TIP3P & nopol & polGS & polSS & polLR & super \\
\hline VMC & $3.637(3)$ & $4.266(3)$ & $4.191(3)$ & $4.435(3)$ & $4.376(3)$ & - & $4.40(2)$ \\
DMC & $3.621(6)$ & $4.232(5)$ & $4.172(5)$ & $4.434(5)$ & $4.396(5)$ & - & $4.30(2)$ \\
CASPT2 & 3.559 & 4.213 & 4.127 & 4.385 & 4.297 & - & 4.224 \\
TDDFT & 3.549 & 4.145 & 4.068 & 4.311 & 4.250 & 4.304 & 4.112 \\
\hline & & & \multicolumn{7}{c}{$\pi \rightarrow \pi^{*}$} \\
VMC & $6.944(3)$ & $6.557(3)$ & $6.608(3)$ & $6.548(3)$ & $6.521(3)$ & - & $6.58(2)$ \\
DMC & $6.771(6)$ & $6.405(5)$ & $6.455(5)$ & $6.392(6)$ & $6.314(6)$ & - & $6.33(2)$ \\
CASPT2 & 6.727 & 6.431 & 6.494 & 6.366 & 6.337 & - & 6.221 \\
TDDFT & 6.150 & 6.038 & 6.057 & 6.008 & 5.994 & 5.913 & 5.831 \\
\hline
\end{tabular}

Table 5.5: Excitation energies (eV) of the $\mathrm{n} \rightarrow \pi^{*}$ and $\pi \rightarrow \pi^{*}$ states of acrolein isolated and solvated in a water cluster. The statistical error on the QMC values is given in brackets.

and DMC ones. Moreover, DMC significantly corrects VMC red-shifting the excitation energy by about $0.1 \mathrm{eV}$ while, for the embedded results, the differences between the two flavors of QMC is of the order of $0.03 \mathrm{eV}$ or smaller.

For the $\pi \rightarrow \pi^{*}$ state, the spread in the excitation energies is particularly large with the correlated values being systematically higher by $0.4-0.6 \mathrm{eV}$ than the corresponding CAM-B3LYP results. Other signatures of the complexity of this state are that the perturbation correction on the zero-order CASSCF excitation energies is more than $1 \mathrm{eV}$ in the isolated system and remains as large as $0.7 \mathrm{eV}$ for solvated acrolein The DMC correction on VMC is around $0.2 \mathrm{eV}$ and therefore larger (albeit the same in the isolated and solvated cases) than what observed so far for MCP and the $\mathrm{n} \rightarrow \pi^{*}$ state of acrolein. We find that CASPT2 and DMC agree in predicting a very comparable red-shift induced by the point charges as well as further changes in the excitation energy due to the different treatments of polarization. This level of agreement is somewhat surprising since the dipoles included in the zero-order CASPT2 Hamiltonian are induced in a CASSCF calculation leading to significantly higher excitation energies, while the dipoles employed in DMC have been optimized accounting for dynamical correlation at the VMC level. In general, all methods confirm that a frozen environment only slightly red-shifts the excitation energies with respect to the point-charge embedding and that differential polarization effects are rather small with an average correction on polGS of about $0.04 \mathrm{eV}$. While electrostatic polarization does not dominate the solute-solvent coupling in this state, other effects can play a role as it might be revealed in a linear response formulation of polarizable embedding. We find in fact that, at the TDDFT level, polLR corrects the polGS excitation energy by about $0.1 \mathrm{eV}$, consistently with the bright nature of the $\pi \rightarrow \pi^{*}$ state. Since both effects should be accounted for in a realistic treatment of the solute-solvent system, one should move beyond a quantum-in-classical description of the $\pi \rightarrow \pi^{*}$ state of acrolein, ultimately extending the correlated quantum calculation to a larger region .

Finally, we note that the QMC supermolecular $\pi \rightarrow \pi^{*}$ excitation energy is higher 

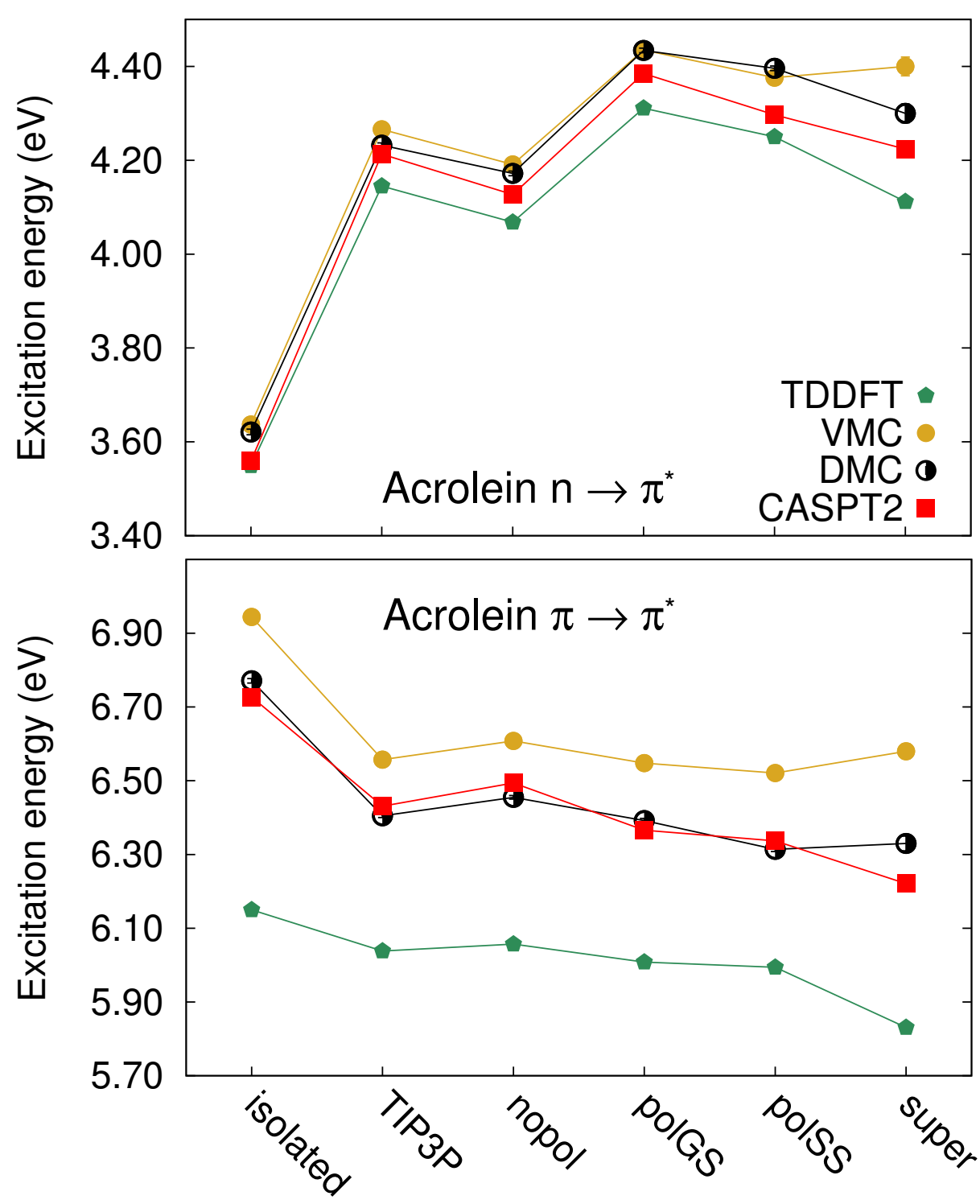

Figure 5.3: Excitation energies of the $\mathrm{n} \rightarrow \pi^{*}$ (top) and $\pi \rightarrow \pi^{*}$ (bottom) state of $s$-trans acrolein with different QM methods and embedding schemes.

than the CASPT2 value even though the two methods agree in all other conditions. If we exclude possible shortcomings of CASPT2 due to the large perturbation correction (about $0.7 \mathrm{eV}$ for embedded acrolein and $0.8 \mathrm{eV}$ in the supermolecular calculation) on the zero-order CASSCF excitation energies, the high supermolecular DMC value could indicate that the trial wave function must be extended to include excitations coupling the solute and the solvent molecules. While these transitions are not included in the reference CASSCF wave functions used in both DMC and CASPT2, they are however present in the perturbation correction and their importance seems to be here confirmed by the improved treatment of the solvent-solute coupling observed at the polLR level. This possibility must be the subject of further investigations. 


\subsection{Conclusions}

In this paper, we combine for the first time quantum Monte Carlo with the selfconsistent optimization of polarizable dipoles (QMC/MMpol) and investigate how such a discrete polarizable model affects the vertical excitation energies of prototypical solvated organic molecules, namely, MCP and acrolein in water. We consider two regimes of polarization where the induced dipoles are optimized with respect to the ground-state density (polGS) and in a state-specific manner (polSS), and assess the quality of the results against a complete quantum (supermolecular) calculation of the same solvated cluster. The induced dipoles optimized with respect to the solute described at the VMC level are then used in a DMC calculation of the excitation energies. We also compare the QMC results obtained in the different embedding schemes against available QM/MMpol implementations of CASPT2 and TDDFT. Within TDDFT we also consider a third way to polarize the environment in a linear response formalism (polLR), where the dipoles equilibrate with the transition density matrix.

We find that our QMC/MMpol approach correctly predicts the response of the excitation energy to the different polarizable environments and, through the inclusion of differential polarization effects, leads in general to an improved description of the excitation with respect to the use of static point charges, in particular when the solute couples mainly electrostatically to the solvent. In MCP and the $n \rightarrow \pi^{*}$ transition of acrolein, we find that improving the description of the ground-state solvent through polGS does not improve (in fact, worsens) the agreement with the reference excitation energies with respect to the use of a standard QM/MM scheme. Introducing a state-specific description of polarization through polSS corrects the shortcomings of polGS and brings the excitation energies in closer agreement with the supermolecular values. On the other hand, as expected, when such electrostatic effects are small as in the case of the $\pi \rightarrow \pi^{*}$ state of acrolein, the polarization schemes developed here are not sufficient as indicated by the better performance of polLR at the TDDFT level and one must move to a correlated description of a larger quantum region to capture the quantum coupling in the excited solute-solvent system. Importantly, this study offers a clear demonstration that, through the comparison of different quantum approaches (QMC, CASPT2, and TDDFT) and the influence of the various embedding schemes (with point charges, polGS, polSS, and polLR), it is possible to gain a deep understanding of the nature of the coupling between the solute and the environment and well characterize the electronic transition in the system of interest.

In summary, our QMC/MMpol scheme in the polGS and polSS flavors with dipoles optimized in the presence of dynamical correlation offers a robust treatment of the different electrostatic effects which might affect the excitation energy. Considering the relatively small computational cost of this hybrid approach and the higher accuracy with respect to the standard point-charge embedding, QMC/MMpol represents a particularly useful tool for the study of larger photoactive (bio)systems of chemical interest, where the specific environment-chromophore interactions play a major role and must be modeled within a realistic framework. 


\section{Bibliography}

[1] A. Warshel and M. Levitt, J. Mol. Biol. 103, 227 (1976).

[2] H. Lin and D. Truhlar, Theor. Chem. Acc. 117, 185 (2007).

[3] H. M. Senn and W. Thiel, Angew. Chem. Int. Ed. 48, 1198 (2009).

[4] Y. Tu and A. Laaksonen, Combining Quantum Mechanics and Molecular Mechanics. Some Recent Progresses in QM/MM Methods, Vol. 59 of Advances in Quantum Chemistry (PUBLISHER, 2010), pp. 1-15.

[5] R. Zhang, B. Lev, J. E. Cuervo, S. Y. Noskov, and D. R. Salahub, Combining Quantum Mechanics and Molecular Mechanics. Some Recent Progresses in QM/MM Methods, Vol. 59 of Advances in Quantum Chemistry (PUBLISHER, 2010), pp. 353-400.

[6] F. H. Wallrapp and V. Guallar, Wiley Interdiscip. Rev. Comput. Mol. Sci. 1, 315 (2011).

[7] B. Mennucci, Phys. Chem. Chem. Phys. 15, 6583 (2013).

[8] J. Gao, Reviews in Computational Chemistry (PUBLISHER, 2007), Vol. 7, pp. 119-185.

[9] G. Seabra, J. Swails, and A. Roitberg, in Multi-scale Quantum Models for Biocatalysis, Vol. 7 of Challenges and Advances in Computational Chemistry and Physics, edited by D. York and T.-S. Lee (PUBLISHER, 2009), pp. 3-20.

[10] J. Tomasi, B. Mennucci, and R. Cammi, Chem. Rev. 105, 2999 (2005).

[11] M. Wanko, M. Hoffmann, P. Strodel, A. Koslowski, W. Thiel, F. Neese, T. Frauenheim, and M. Elstner, J. Phys. Chem. B 109, 3606 (2005).

[12] M. Wanko, M. Hoffmann, T. Frauenheim, and M. Elstner, J. Phys. Chem. B 112, 11462 (2008).

[13] M. Wanko, M. Hoffmann, J. Frähmcke, T. Frauenheim, and M. Elstner, J. Phys. Chem. B 112, 11468 (2008).

[14] R. Send, V. R. I. Kaila, and D. Sundholm, J. Chem. Phys. 134, 214114 (2011). 
[15] K. Sneskov, T. Schwabe, O. Christiansen, and J. Kongsted, Phys. Chem. Chem. Phys. 13, 18551 (2011).

[16] T. Schwabe, J. M. H. Olsen, K. Sneskov, J. Kongsted, and O. Christiansen, J. Chem. Theory Comput. 7, 2209 (2011).

[17] C. Filippi, F. Buda, L. Guidoni, and A. Sinicropi, J. Chem. Theory Comput. 8, $112(2012)$

[18] C. M. Isborn, A. W. Götz, M. A. Clark, R. C. Walker, and T. J. Martínez, J. Chem. Theory Comput. 8, 5092 (2012).

[19] P. Amat and R. Nifosi, J. Chem. Theory Comput. 9, 497 (2013).

[20] T. Wolter, K. Welke, P. Phatak, A.-N. Bondar, and M. Elstner, Phys. Chem. Chem. Phys. 15, 12582 (2013).

[21] O. Valsson, P. Campomanes, I. Tavernelli, U. Rothlisberger, and C. Filippi, J. Chem. Theory Comput. 9, 2441 (2013).

[22] V. R. I. Kaila, R. Send, and D. Sundholm, Phys. Chem. Chem. Phys. 15, 4491 (2013).

[23] T. Schwabe, M. T. P. Beerepoot, J. M. H. Olsen, and J. Kongsted, Phys. Chem. Chem. Phys. 17, (2015).

[24] R. Send, C.-M. Suomivuori, V. R. I. Kaila, and D. Sundholm, J. Phys. Chem. B 119, 2933 (2015).

[25] C. Daday, C. Curutchet, A. Sinicropi, B. Mennucci, and C. Filippi, J. Chem. Theory Comput. 11, 4825 (2015).

[26] M. A. Thompson, J. Phys. Chem. 100, 14492 (1996).

[27] C. Curutchet, A. Muñoz-Losa, S. Monti, J. Kongsted, G. D. Scholes, and B. Mennucci, J. Chem. Theory Comput. 5, 1838 (2009).

[28] J. M. Olsen, K. Aidas, and J. Kongsted, J. Chem. Theory Comput. 6, 3721 (2010).

[29] L. V. Slipchenko, J. Phys. Chem. A 114, 8824 (2010).

[30] K. Sneskov, T. Schwabe, J. Kongsted, and O. Christiansen, J. Chem. Phys. 134, 104108 (2011).

[31] K. Sneskov, J. M. H. Olsen, T. Schwabe, C. Hattig, O. Christiansen, and J. Kongsted, Phys. Chem. Chem. Phys. 15, 7567 (2013).

[32] Q. Li, B. Mennucci, M. A. Robb, L. Blancafort, and C. Curutchet, J. Chem. Theory Comput. 11, 1674 (2015). 
[33] L. Jensen, P. T. van Duijnen, and J. G. Snijders, J. Chem. Phys. 119, 3800 (2003).

[34] M. T. P. Beerepoot, A. H. Steindal, J. Kongsted, B. O. Brandsdal, L. Frediani, K. Ruud, and J. M. H. Olsen, Phys. Chem. Chem. Phys. 15, 4735 (2013).

[35] A. H. Steindal, J. M. H. Olsen, K. Ruud, L. Frediani, and J. Kongsted, Phys. Chem. Chem. Phys. 14, 5440 (2012).

[36] M. T. Beerepoot, A. H. Steindal, K. Ruud, J. M. Olsen, and J. Kongsted, Comp. Theor. Chem. 1040-1041, 304 (2014).

[37] A. Pikulska, A. H. Steindal, M. T. P. Beerepoot, and M. Pecul, J. Phys. Chem. B 119, 3377 (2015).

[38] C. Filippi, M. Zaccheddu, and F. Buda, J. Chem. Theory Comput. 5, 2074 (2009).

[39] O. Valsson and C. Filippi, J. Chem. Theory Comput. 6, 1275 (2010).

[40] R. Send, O. Valsson, and C. Filippi, J. Chem. Theory Comput. 7, 444 (2011).

[41] O. Valsson, C. Angeli, and C. Filippi, Phys. Chem. Chem. Phys. 14, 11015 (2012).

[42] C. Daday, C. König, J. Neugebauer, and C. Filippi, ChemPhysChem. 15, 3205 (2014).

[43] B. T. Thole and P. T. van Duijnen, Theor. Chim. Acta 55, 307 (1980).

[44] P. T. van Duijnen and M. Swart, J. Phys. Chem. A 102, 2399 (1998).

[45] M. Masia, M. Probst, and R. Rey, J. Chem. Phys. 123, (2005).

[46] J. Wang, P. Cieplak, J. Li, T. Hou, L. Ray, and D. Yong, J. Chem. Phys. B 8, 3091 (2011).

[47] We note that, in a QMC calculation, the electronic contribution of the solute to the electric field at $\mathbf{r}_{\mathrm{a}}$ is computed by sampling the square of the many-body wave function and collecting the average of $\sum_{i}\left(\mathbf{r}_{\mathrm{a}}-\mathbf{r}_{i}\right) /\left|\mathbf{r}_{i}-\mathbf{r}_{\mathrm{a}}\right|^{3}$, where the sum is over the electrons.

[48] C. J. Umrigar, J. Toulouse, C. Filippi, S. Sorella, and R. G. Hennig, Phys. Rev. Lett. 98, 110201 (2007).

[49] CHAMP is a quantum Monte Carlo program package written by C. J. Umrigar, C. Filippi, S. Moroni, and collaborators.

[50] M. Burkatzki, C. Filippi, and M. Dolg, J. Chem. Phys. 126, 234105 (2007). 
[51] For the hydrogen atom, we use a more accurate BFD pseudopotential and basis set. Dolg, M.; Filippi, C., private communication.

[52] M. W. Schmidt, K. K. Baldridge, J. A. Boatz, S. T. Elbert, M. S. Gordon, J. H. Jensen, S. Koseki, N. Matsunaga, K. A. Nguyen, S. Su, T. L. Windus, M. Dupuis, and J. A. M. Jr, J. Comput. Chem. 14, 1347 (1993).

[53] M. S. Gordon and M. W. Schmidt, in Theory and Applications of Computational Chemistry: the first forty years, edited by C. Dykstra, G. Frenking, K. Kim, and G. Scuseria (Elsevier, 2011), Chap. 41, pp. 1167-1190.

[54] C. Filippi and C. J. Umrigar, J. Chem. Phys. 105, 213 (1996), as Jastrow correlation factor, we use the exponential of the sum of three fifth-order polynomials of the electron-nuclear (e-n), the electron-electron (e-e). The Jastrow factor is adapted to deal with pseudo-atoms, and the scaling factor $\kappa$ is set to 0.6 a.u.The 2-body Jastrow factor includes five parameters in the e-e terms and four parameters for each atom type in the e-n terms.

[55] M. Casula, Phys. Rev. B 74, 161102 (2006).

[56] F. Aquilante, L. De Vico, N. Ferré, G. Ghigo, P.-å. Malmqvist, P. Neogrády, T. B. Pedersen, M. Pitoňák, M. Reiher, B. O. Roos, L. Serrano-Andrés, M. Urban, V. Veryazov, and R. Lindh, J. Comput. Chem. 31, 224 (2010).

[57] K. Andersson, P.-A. Malmqvist, B. O. Roos, A. J. Sadlej, and K. Wolinski, J. Phys. Chem. 94, 5483 (1990).

[58] K. Andersson, P.-A. Malmqvist, and B. O. Roos, J. Chem. Phys. 96, 1218 (1992).

[59] G. Ghigo, B. O. Roos, and P.-Å. Malmqvist, Chem. Phys. Lett. 396, 142 (2004).

[60] N. Forsberg and P.-Å. Malmqvist, Chem. Phys. Lett. 274, 196 (1997).

[61] M. J. Frisch, G. W. Trucks, H. B. Schlegel, G. E. Scuseria, M. A. Robb, J. R. Cheeseman, G. Scalmani, V. Barone, B. Mennucci, G. A. Petersson, H. Nakatsuji, M. Caricato, X. Li, H. P. Hratchian, A. F. Izmaylov, J. Bloino, G. Zheng, J. L. Sonnenberg, M. Hada, M. Ehara, K. Toyota, R. Fukuda, J. Hasegawa, M. Ishida, T. Nakajima, Y. Honda, O. Kitao, H. Nakai, T. Vreven, J. A. Montgomery, Jr., J. E. Peralta, F. Ogliaro, M. Bearpark, J. J. Heyd, E. Brothers, K. N. Kudin, V. N. Staroverov, R. Kobayashi, J. Normand, K. Raghavachari, A. Rendell, J. C. Burant, S. S. Iyengar, J. Tomasi, M. Cossi, N. Rega, J. M. Millam, M. Klene, J. E. Knox, J. B. Cross, V. Bakken, C. Adamo, J. Jaramillo, R. Gomperts, R. E. Stratmann, O. Yazyev, A. J. Austin, R. Cammi, C. Pomelli, J. W. Ochterski, R. L. Martin, K. Morokuma, V. G. Zakrzewski, G. A. Voth, P. Salvador, J. J. Dannenberg, S. Dapprich, A. D. Daniels, Ö. Farkas, J. B. Foresman, J. V. Ortiz, J. Cioslowski, and D. J. Fox, Gaussian 09 Revision A.02, Gaussian Inc. Wallingford CT 2009. 
[62] T. Yanai, D. P. Tew, and N. C. Handy, Chem. Phys. Lett. 393, 51 (2004).

[63] Carles Curutchet, private communication.

[64] J. Wang, P. Cieplak, J. Li, J. Wang, Q. Cai, M. Hsieh, H. Lei, R. Luo, and Y. Duan, J. Chem. Phys. B 8, 3100 (2011).

[65] W. L. Jorgensen, J. Chandrasekhar, J. D. Madura, R. W. Impey, and M. L. Klein, J. Chem. Phys. 79, 926 (1983).

[66] A. D. Becke, Phys. Rev. A 38, 3098 (1988).

[67] C. Lee, W. Yang, and R. G. Parr, Phys. Rev. B 37, 785 (1988).

[68] We include diffuse functions only for the heavy atoms and take them from the aug-cc-pVXZ basis sets in the EMSL Basis Set Library (http://bse.pnl.gov). For the double and triple $\zeta$ basis sets, we omit the $p$ and $f$ diffuse functions, respectively.

[69] T. H. Dunning Jr, J. Chem. Phys. 90, 1007 (1989).

[70] K. A. Peterson, D. E. Woon, and T. H. Dunning Jr, J. Chem. Phys. 100, 7410 (1994).

[71] A. Wilson, T. van Mourik, and T. H. Dunning Jr, J. Mol. Struct. (Theochem) 388, 339 (1997).

[72] F. Weigend, Phys. Chem. Chem. Phys. 8, 1057 (2006).

[73] S. Corni, R. Cammi, B. Mennucci, and J. Tomasi, J. Chem. Phys. 123, 134512 (2005).

[74] S. W. Staley and T. D. Norden, J. Am. Chem. Soc. 106, 3699 (1984).

[75] A. Moskvin, O. Yablonskii, and L. Bondar, Theor. Exp. Chem. 2, 469 (1966).

[76] T. D. Norden, S. W. Staley, W. H. Taylor, and M. D. Harmony, J. Am. Chem. Soc. 108, 7912 (1986).

[77] M. Merchán, R. González-Luque, and B. O. Roos, Theor. Chim. Acta 94, 143 (1996).

[78] A. M. Losa, I. F. Galván, M. A. Aguilar, and M. E. Martín, J. Phys. Chem. B 111, 9864 (2007).

[79] S. A. do Monte, T. Müller, M. Dallos, H. Lischka, M. Diedenhofen, and A. Klamt, Theor. Chem. Acc. 111, 78 (2004). 


\section{Chapter 6}

\section{Dissecting polarization in photoexcited rhodopsin: Quantum effects from classical modeling ${ }^{\dagger}$}

We investigate here how the light absorption of the retinal chromophore is regulated in rhodopsin by the protein environment and rationalize the nature of the coupling of the chromphore with its surrounding in the photoexcitation process. We employ classical but polarizable embedding via induced dipoles to recover a description of the chromophore-protein system that not only accurately accounts for electrostatic and induction interactions but also includes non-classical resonance effects. We also show that it is possible to use the same analysis to fingerprint the role of the surrounding amino acids in determining the final spectroscopic response of the embedded chromophore. We find that, while accounting for the electrostatic relaxation of the environment in response to the excitation of retinal is important, one needs to include resonance effects between the chromophore and several close amino acids for a complete description of the absorption process: The same residues which more strongly polarize in the excited state of the chromophore also "resonate" to its transition density when treated within a linear-response scheme. Our analysis therefore demonstrates that the protein-chromophore interactions in rhodopsin are not only electrostatic and that the use of polarizable dipoles is an effective scheme to also mimic resonance coupling without resorting to an explicit quantum calculation of an extended region around the chromophore.

$\dagger$ This chapter is in preparation as R. Guareschi, O. Valsson, C. E. Curutchet, B. Mennucci, and C. Filippi, "Dissecting polarization in photoexcited rhodopsin: Quantum effects from classical modeling", 


\subsection{Introduction}

Visual opsins regulate the process of vision at the molecular level and have become a prime playground for the understanding and further manipulation of how a protein environment controls the light-activated response of an embedded photosensitive species [1-3]. It is remarkable that most members of this large family of receptors share the same photosensitive component, the 11-cis retinal protonated Schiff base (RPSB) chromophore (Fig. 6.1), and that relatively minor variations in the protein surroundings can tune its absorption over a spectral range as wide as $420-570 \mathrm{~nm}$. This is the result of multiple interactions between the chromophore and the protein whose fundamental nature, however, has so far eluded our understanding. Rationalizing the essence of the chromophore-protein coupling and, consequently, the regulatory mechanism of absorption in opsins, amounts to answering few precise questions: Are these interactions purely electrostatic or do we need to account for more subtle physical effects? Can we identify the amino acids active in the excitation process and the character of their contribution? Is their participation amenable to the same theoretical description?

Given the complexity of the system, multiscale approaches to bridge between the smaller scales of the chromophore and the larger protein environment have been commonly employed to study the absorption properties of opsins. However, when opting for a particular multiscale scheme, one has implicitly acknowledged to know the correct answer to some of these questions: For instance, the common use of static force field automatically relegates the protein environment to a mere spectator when light shines on it. To overcome the limitations inherent in the choice of any multiscale scheme, recent theoretical studies have extended the quantum treatment to larger regions and suggested that at least one shell of amino acids in proximity of the RPSB chromophore participates in the photo-induced process $[4,5]$. These findings are qualitatively in line with earlier pioneering studies $[6,7]$ on prototypical opsins by Elstner and coworkers, who found the environment to electrostatically respond to the photoexcitation of the chromophore when relaxed in a state-specific manner using either a quantum-in-quantum partition or through polarizable force fields. In all these cases, the observed effects were loosely classified as "polarization" but is the polarization captured by a responsive (quantum or classical) treatment of the environment the same one recovered in the quantum calculation of a large photoexcited region? To date, the true identity of these effects in opsins has not been uncovered.

Here, we will give physical content to the somewhat ambiguous term of polarization and show how it is possible to fingerprint the role of the amino acids surrounding the RPSB chromophore in determining absorption. We focus our analysis on the archetype of visual opsins, namely, bovine rhodopsin, which is responsible for dim-light vision and the most extensively studied member of this protein family both experimentally $[1,3,8-11]$ and theoretically [12-33]. This protein exhibits a strong absorption centered at $2.49 \mathrm{eV}$, dominated by the $\pi \rightarrow \pi^{*}$ transition on the RPSB chromophore, and associated with electronic charge transfer from the $\beta$-ionone ring towards the terminal side of the conjugated chain. From qualitative considerations, one expects that the electrostatic interaction of the positive chromophore with the 
Glu113 counterion (Fig. 6.1), induces a blue-shift in absorption with respect to the gas phase. The size of this shift and the role played by the rest of the protein in compensating this shift has been much debated over the years with estimates for both effects often differing considerably in magnitude [13, 16, 19-26, 29,30]. With the few exceptions already noted above, most studies have limited the photoexcitation to the chromophore and froze the environment as a computationally affordable, yet static, sea of classical point charges in a quantum-mechanics-in-molecularmechanics (QM/MM) calculation.

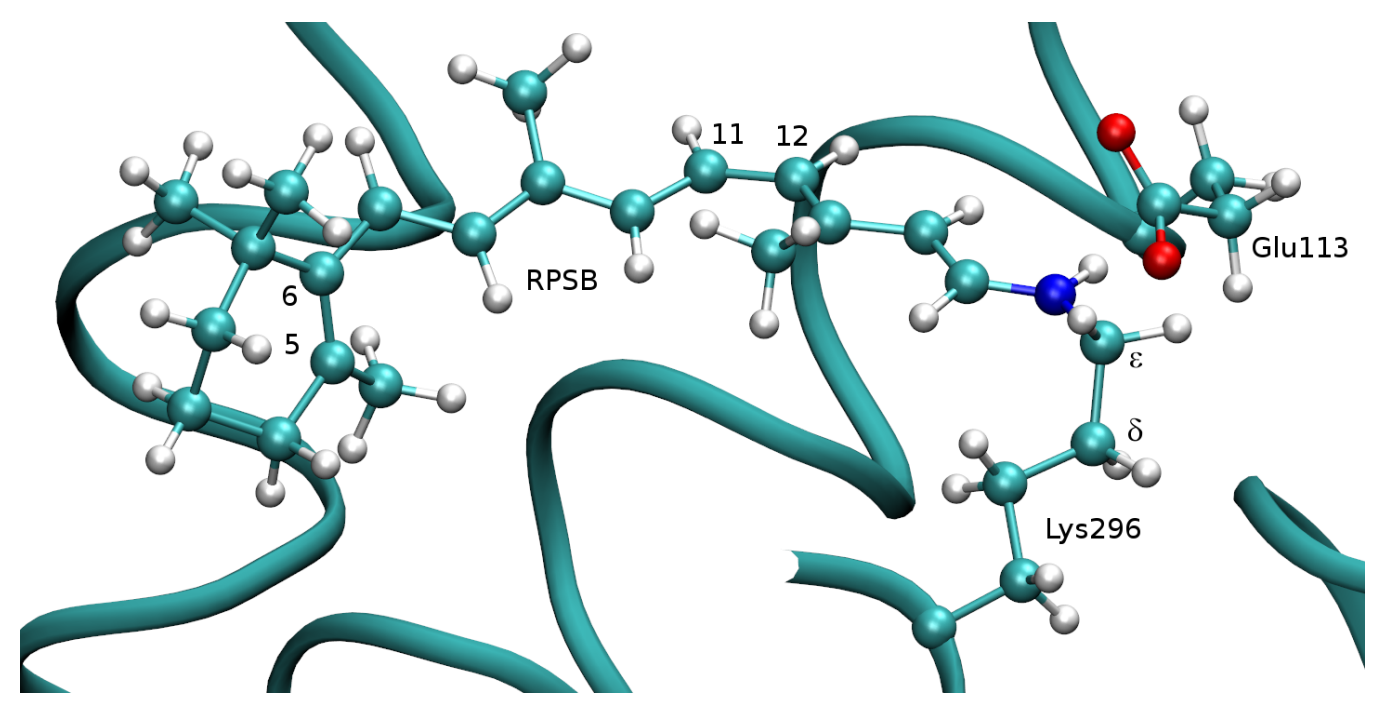

Figure 6.1: The RPSB chromophore with the Glu113 counterion. We also show the side chain of the Lys296 residue covalently linked to the chromophore.

To understand how the chromophore and the protein couple in rhodopsin absorption, we need a theoretical approach which has the flexibility to selectively recover the (static and responsive) electrostatic interaction of photoexcited RPSB with its protein pocket and the intrinsically quantum effects which go beyond electrostatic embedding. The use of an extended quantum region, for instance within timedependent density functional theory (TDDFT) or cheaper correlated approaches, allows us to establish reference target values for the excited-state properties of interest at the chosen level of theory but ultimately hinders the analysis we want to carry out here, hiding inside its complete description the nature of the interactions. It has however been often overlooked that, somewhat surprisingly, it is possible to employ a quantum description for a photoexcited site coupled to a classical polarizable surrounding medium not only to recover the dominant electrostatic interactions but also to mimic non-classical resonance interactions between the active region and the rest of the system. If we maintain a discrete classical treatment of the protein, the use of a set of charges in combination with induced dipoles in a so-called QM/MMpol approach represents a very powerful tool for our purposes since polarization effects can be introduced following different schemes which consent to capture different components of the coupling. 


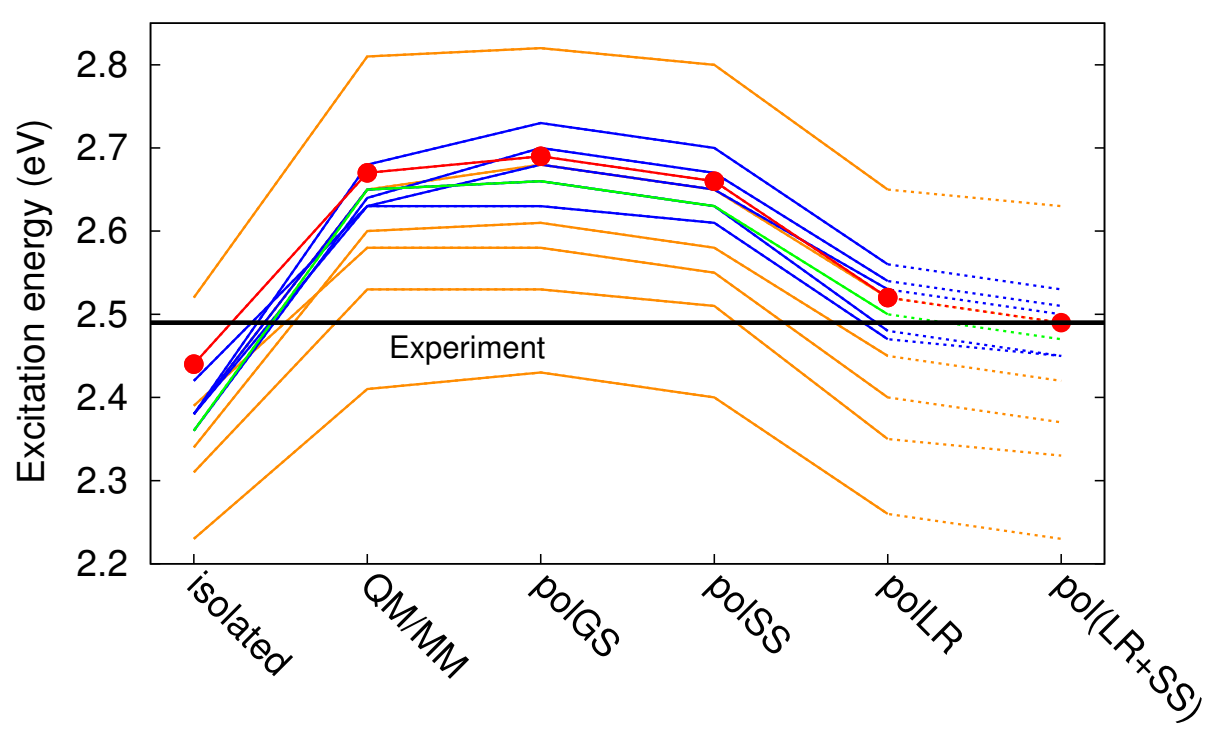

Figure 6.2: TDDFT excitation energies (eV) computed for RPSB isolated and in a rhodopsin environment described with static point charges (QM/MM) and with dipoles induced in the ground state (polGS), in a state-specific (polSS), and in a linear-response formulation (polLR). The orange lines identify the frames (A) from cluster analysis, the blue and green lines the frames from the center of the ZINDO spectrum (B and C), and the red circles an annealed frame. The experimental absorption maximum is at $2.49 \mathrm{eV}$.

\subsection{Results}

We begin our analysis of rhodopsin from the TDDFT excitation energies computed with the CAM-B3LYP exchange-correlation functional [34] using three MMpol schemes to selectively introduce different types of interactions between the RPSB chromophore and the protein. First, we polarize the dipoles to the ground-state density of RPSB and keep them unchanged in the computation of the excitation energy (polGS). Then, state-specific dipoles are induced to account for possible differential electrostatic effects between ground and excited states (polSS). Given the large transfer of electronic charge following photoexcitation, one would expect a strong electrostatic interaction of RPSB with its surrounding and a sizable polSS correction on the polGS excitation energies (a rather small shift, however, was previously reported for one structural realization of the system). In the last scheme, we adopt a linear-response framework (polLR), where the induced dipoles respond to the transition density of the photoexcited species. This embedding condition accounts for the non-classical part of the coupling between the chromophore and the environment, does not include the electrostatic response, and has been shown to correspond to a dispersion-like coupling between the quantum system that undergoes to the excitation and the classical dipoles of the environment [35].

The results are summarized in Fig. 6.2. To account for temperature effects and 
avoid the risk of limiting the analysis to a single and possibly not representative structure, we consider here four groups of frames extracted according to different recipes from long QM/MM molecular dynamics (MD) trajectories, where the QM part is treated within density functional theory (DFT) and the PBE exchange-correlation functional [36]. Frames A (frames 1-6) are the central frames of the most populated clusters obtained in a cluster analysis of a trajectory we generated with the chromophore and the counterion Glu113 in the QM region. Frames B (frames 7-11) are close to the center of the ZINDO spectrum previously computed for a different trajectory obtained in a similar manner [4]. Frame $\mathrm{C}$ (frame 12) is from the same study and selected from the center of the ZINDO spectrum of a QM/MM MD simulation where the QM region includes also the anionic residue Glu181. Finally, we also determine an annealed structure from the trajectory from which frames A are extracted.

The excitation energies of all snapshots follow precisely the same trend: The inclusion of static point charges determines a significant blue shift of the excitation energy (about $0.2 \mathrm{eV}$ ) with respect to the values computed on the isolated chromophore at the geometry extracted from the protein. This can be mostly attributed to the presence of the counterion stabilizing the ground state and inhibiting the charge transfer in the excited state. When the chromophore is embedded in a polarizable environment equilibrated on the ground state of RPSB (polGS), the excitation energies become somewhat larger as a consequence of the further stabilization of the ground state with respect to the excited state. The effect of this improved ground-state description of the system is however rather small at the TDDFT level, being at most $0.06 \mathrm{eV}$ and in average $0.02 \mathrm{eV}$. If we further enhance the electrostatic description of the chromophore-protein coupling by introducing differential polarization effects through state-specific dipoles, we recover a red-shift in the polSS excitation energies with respect to the polGS values, which is however rather small and consistently between -0.03 and $-0.02 \mathrm{eV}$. The presence of a polarizable environment has a much larger effect on the absorption energies only when the dipoles are induced in the linear response regime (polLR), namely, in response to the transition density associated with the electronic excitation. The non-classical part of the coupling captured in these calculations appears therefore to play a dominant role, lowering the excitation energies by as much as $0.16-0.18 \mathrm{eV}$ with respect to the polGS value and largely recovering the so-called counterion quenching which was lost in an electrostatic (static and responsive) treatment of the protein.

To better understand qualitatively the origin of these shifts and their quantitative dependence on the quantum approach (so far TDDFT) chosen to describe RPSB, we recall that, perturbatively to linear order, the polSS and polLR corrections with respect to the polGS excitation energies are proportional to the square of the variation of the molecular dipole between ground and excited states, and to the transition dipole moment, respectively, so that [37]

$$
\frac{\Delta E_{\mathrm{exc}}^{(\mathrm{SS}-\mathrm{GS})}}{\Delta E_{\mathrm{exc}}^{(\mathrm{LR}-\mathrm{GS})}} \approx \frac{|\Delta \boldsymbol{\mu}|^{2}}{2\left|\boldsymbol{\mu}_{01}\right|^{2}},
$$

with $\Delta E_{\mathrm{exc}}^{(\mathrm{XX}-\mathrm{GS})}=E_{\mathrm{exc}}^{\mathrm{polXX}}-E_{\mathrm{exc}}^{\mathrm{polGS}}$. If we first focus on the state-specific contribution, we observe that we would have expected a more sizable reduction of the 


\begin{tabular}{lccccc}
\hline Frame & $\left|\boldsymbol{\mu}_{01}\right|$ & $|\Delta \boldsymbol{\mu}|$ & $\Delta \mathrm{E}_{\mathrm{exc}}^{(\mathrm{SS}-\mathrm{GS})}$ & $\Delta \mathrm{E}_{\mathrm{exc}}^{(\mathrm{LR}-\mathrm{GS})}$ & $\Delta \mathrm{E}_{\mathrm{exc}}^{(\mathrm{LR}-\mathrm{GS})} \frac{|\Delta \boldsymbol{\mu}|^{2}}{2 \boldsymbol{\mu} \boldsymbol{\mu}_{01}^{2}}$ \\
\hline 4 & 11.86 & 6.89 & -0.0340 & -0.1598 & -0.0270 \\
8 & 11.87 & 4.71 & -0.0178 & -0.1583 & -0.0125 \\
12 & 11.83 & 6.18 & -0.0278 & -0.1598 & -0.0218 \\
ann. & 12.26 & 6.32 & -0.0259 & -0.1665 & -0.0221 \\
\hline
\end{tabular}

Table 6.1: TDDFT excitation energy differences (eV) of rhodopsin computed in an environment polarized in a state-specific (polSS) and linear-response (polLR) scheme, relative to the polGS values. The transition dipole moment, $\left|\boldsymbol{\mu}_{01}\right|$, and the change in the dipole moment, $|\Delta \boldsymbol{\mu}|$, obtained within QM/MM are listed in Debye.

excitation energy due to the inclusion of differential polarization effects: The excited state of RPSB displays a strong charge redistribution from the $\beta$-ionone ring to the nitrogen terminus which should be greatly stabilized by the relative optimization of the chromophore surroundings. The difference between the ground- and the excitedstate molecular dipole moment is in fact experimentally rather large and of the order of 12-13 Debye [38,39]. The underestimation of this electrostatic differential contribution in rhodopsin can be easily explained as resulting from an error of approximate TDDFT/CAM-B3LYP, which tends to underestimate the changes of dipole moments in charge-transfer electronic transitions [40]. As shown in Table 6.1, our calculations for rhodopsin are affected by the same problem and yield values of $|\Delta \boldsymbol{\mu}|$ computed in point-charge embedding that are too low by about a factor of two. This incorrectly predicted change in the dipole moments translates in an underestimation by about a factor of four of the electrostatic differential polarization contributions in the TDDFT excitation energies as further discussed below in comparison with correlated calculations.

The polLR corrections to the polGS excitation energies are instead proportional to the magnitude square of the transition dipole moment and, therefore, to the large oscillator strength of the bright $\pi \rightarrow \pi^{*}$ transition of RPSB in rhodopsin. The TDDFT transition dipole moments of about 12 Debye (see Table 6.1) lead in fact to sizable polLR contributions to the excitation energies. In this case, the estimates are reliable and do not suffer from particular shortcomings of the use of TDDFT/CAMB3LYP since the method has generally been shown to produce reasonably good oscillator strengths [41]. We stress that, as shown in Table 6.1, the validity of this perturbative analysis of the polarization contributions to the excitation energies and their relation to the (transition) dipole moments is strongly corroborated in rhodopsin by the remarkable correspondence between the polSS contributions and the values obtained by rescaling the polLR corrections as in Eq. 6.1.

The polLR and polSS schemes account therefore for different components of the chromophore-protein interaction that co-exist in rhodopsin and can be captured in a quantum calculation of a sufficiently large region including RPSB and its surroundings. We can extrapolate the combined effects of these polarization treatments by adding the two corrections on the polGS excitation energies as shown in Fig. 6.2. Such an additive approach appears be appropriate for rhodopsin, being supported by 
these perturbative arguments and the comparison with TDDFT calculations on large clusters. The extrapolated excitation energies are reduced by about $0.2 \mathrm{eV}$ with respect to the polGS values and, for the snapshots energetically close to the annealed one, in good agreement with the experimental absorption maximum. Nevertheless, we should not attribute too much significance in this agreement: We must recall that the state-specific contribution is underestimated in TDDFT, resulting in a blue-shift which is fortuitously compensated by the use of an RPSB geometry computed with a generalized gradient approximation, yielding a smaller bond length alternation and a consequent red-shift. Furthermore, we are neglecting vibronic effects which are not negligible in photoactive rhodopsin and should result in the computed vertical excitation energy being higher than the absorption maximum.

Let us keep our main goal to elucidate the nature of polarization in the coupling between the RPSB chromophore and the rest of the protein. Our MMpol calculations allows us to further our analysis and identify which residues respond more strongly and whether they polarize to the ground state, the excited state or in a resonance fashion. In Fig. 6.3, we show for one frame the dominant contributions to the groundstate polarization $\left(\mathrm{E}_{\mathrm{pol}}^{\mathrm{GS}}\right)$ and the linear contributions induced at the polSS and polLR level relative to polGS:

$$
\delta \mathrm{E}_{\mathrm{pol}}^{\mathrm{XX}}=-\frac{1}{2} \sum_{a} \boldsymbol{\mu}_{a}\left(\rho^{\mathrm{XX}}\right) \cdot \mathbf{E}_{a}\left(\rho^{\mathrm{XX}}\right)
$$

where the sum is over the classical polarizable sites and the dipoles are induced either by the transition density, $\rho^{\mathrm{LR}}=\rho_{01}$, or by the density difference, $\rho^{\mathrm{SS}}=\Delta \rho$, between the ground and excited states of RPSB. The same density distribution also determines the static electric field at the classical sites. For a set of frames, we then determine the residues with contributions to $\delta \mathrm{E}_{\mathrm{pol}}$ larger than about $0.09 \mathrm{kcal} / \mathrm{mol}$. In the polSS case, since the polSS correction to polGS is underestimated by about a factor of 4 as discussed above, we identify the residues whose polSS contributions rescaled by the same factor is above the chosen threshold. In Fig. 6.4, we show the position of the common set of surviving residues, which nicely embrace the RPSB chromophore.

It is first important to appreciate that the dominating contributions to the groundstate polarization are mostly carried by few residues in proximity of the nitrogen end of RPSB, in particular, the covalently linked Lys296, the counterion Glu113, a water molecule occasionally hydrogen-bonded to it, and Ser186, while the response effects in the excited state are spread over a larger number of residues surrounding also the $\beta$-ionone ring. As expected, the polSS and polLR responses correlate rather well: The residues with a strong electrostatic coupling with the chromophore in the excited state are the ones that predominantly resonate with the chromophore at the polLR level. The relative contributions of the different residues changes however between the two polarization schemes. In addition to the three obvious candidates close to the positive terminus of RPSB (Lys286, Glu113, Ser186), the residues displaying a sizable $\pi$ electronic system (Trp265, Tyr268, Phe293, Phe91) contribute the most to the polLR response, with $\operatorname{Trp} 265 \pi$-stacked to one end of the chromophore giving the absolute largest value in several frames. A few residues close to the central 

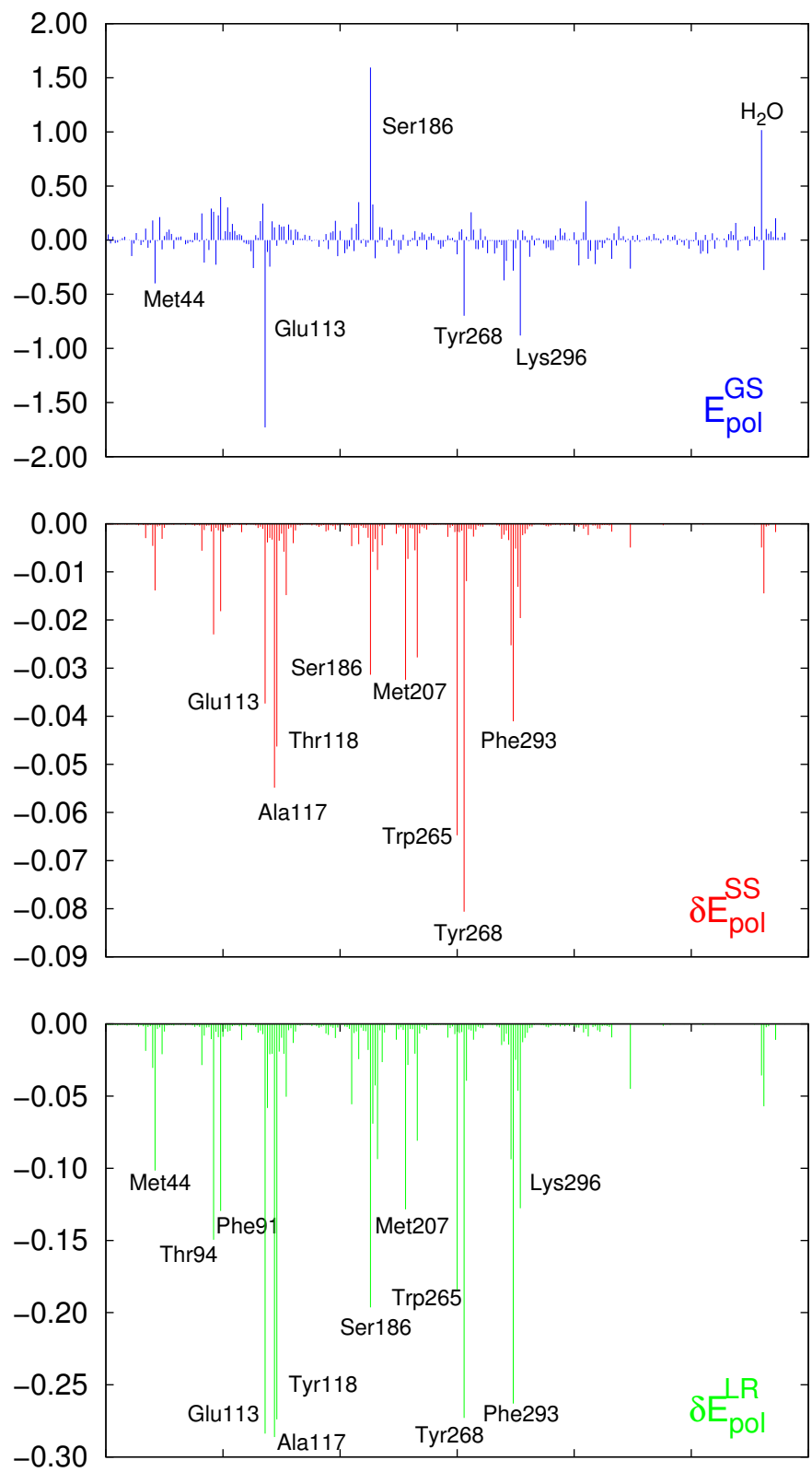

Figure 6.3: Contributions $(\mathrm{kcal} / \mathrm{mol})$ to the ground-state DFT polarization energy $\left(\mathrm{E}_{\mathrm{pol}}^{\mathrm{GS}}\right)$ of all residues within $15 \AA$ of RPSB (top) and relative TDDFT contributions $\left(\delta \mathrm{E}_{\mathrm{pol}}\right)$ computed within the polSS (middle) and polLR (bottom) schemes for frame 4.

part of the conjugated skeleton of the chromophore (Ala117, Tyr118) further stabilize the excitation. We note that the validity of this analysis, based on perturbation arguments (Eq. 6.2), to pinpoint the role of the various residues is strongly supported by the close equality recovered between $\delta \mathrm{E}_{\text {pol }}^{\mathrm{LR}}$ and the excitation energy difference $\Delta \mathrm{E}_{\text {exc }}^{\mathrm{LR}-\mathrm{GS}}$.

The understanding gained so far appears to be sound (and internally consistent) 


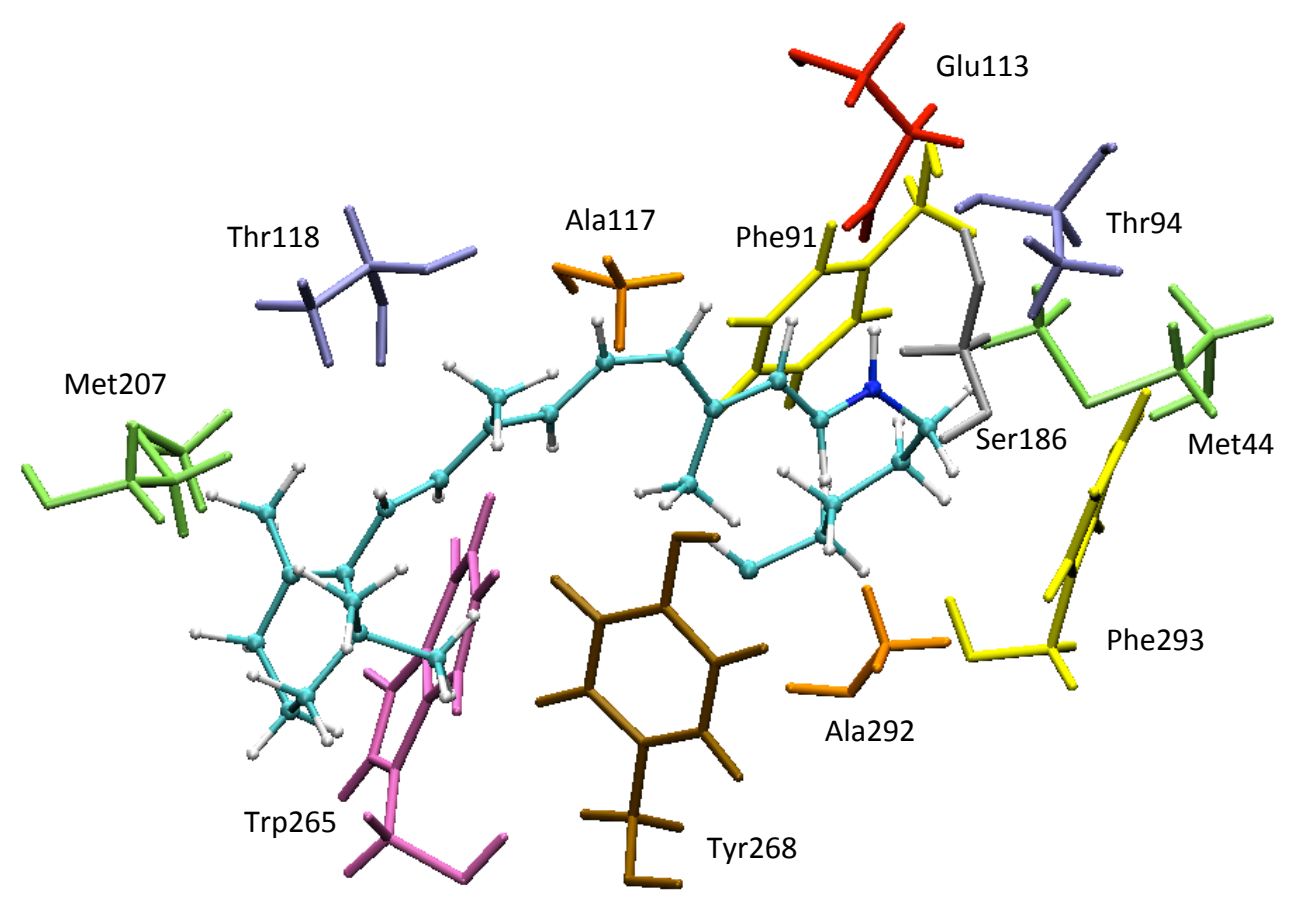

Figure 6.4: The RPSB chromophore covalently linked to Lys296 and the residues with the largest contributions $\delta \mathrm{E}_{\text {pol }}$ to the relative polarization energies in the polSS and polLR schemes. The residues are cut at $\mathrm{C}_{\alpha}$.

within the limitations of TDDFT, which we already know for instance to underestimate electrostatic differential polarization effects. To further validate our observations, we also employ a correlated description of the QM region and select three frames $(4,8$, and 12$)$ to be treated with the complete-active-space self-consistentfield (CASSCF) approach followed by second-order perturbation theory (CASPT2). The comparison between the CASPT2 and TDDFT excitation energies are shown in Fig. 6.5 for one frame and summarized in Table 6.2. The dipoles are induced in a CASSCF calculation targeting either the ground or the excited state, and used unchanged in the PT2 computation of the polGS and polSS excitation energies.

The dependence of the CASPT2 excitation energies on the choice of electrostatic model for the environment follows a similar trend to the one observed at the TDDFT level but the magnitude of the shifts induced by the different descriptions of the environment is much larger. The use of a ground-state-polarized protein raises the excitation energies with respect to point-charge embedding by more than $0.1 \mathrm{eV}$ and accounting for differential polarization effects in the polSS scheme yields a correction of the same magnitude and opposite sign. The change in the correlated excitation energies computed with state-specific dipoles with respect to the polGS values is much larger than in the TDDFT case as a consequence of the more accurate estimation of $|\Delta \boldsymbol{\mu}|$ at the CASPT2 level. In fact, the CASPT2 differences $\Delta \mathrm{E}_{\text {exc }}^{(\mathrm{SS}-\mathrm{GS})}$ can be remarkably well estimated by simply rescaling the corresponding TDDFT values by the ratio squared between the CASPT2 and TDDFT $|\Delta \mu|$, again supporting the 


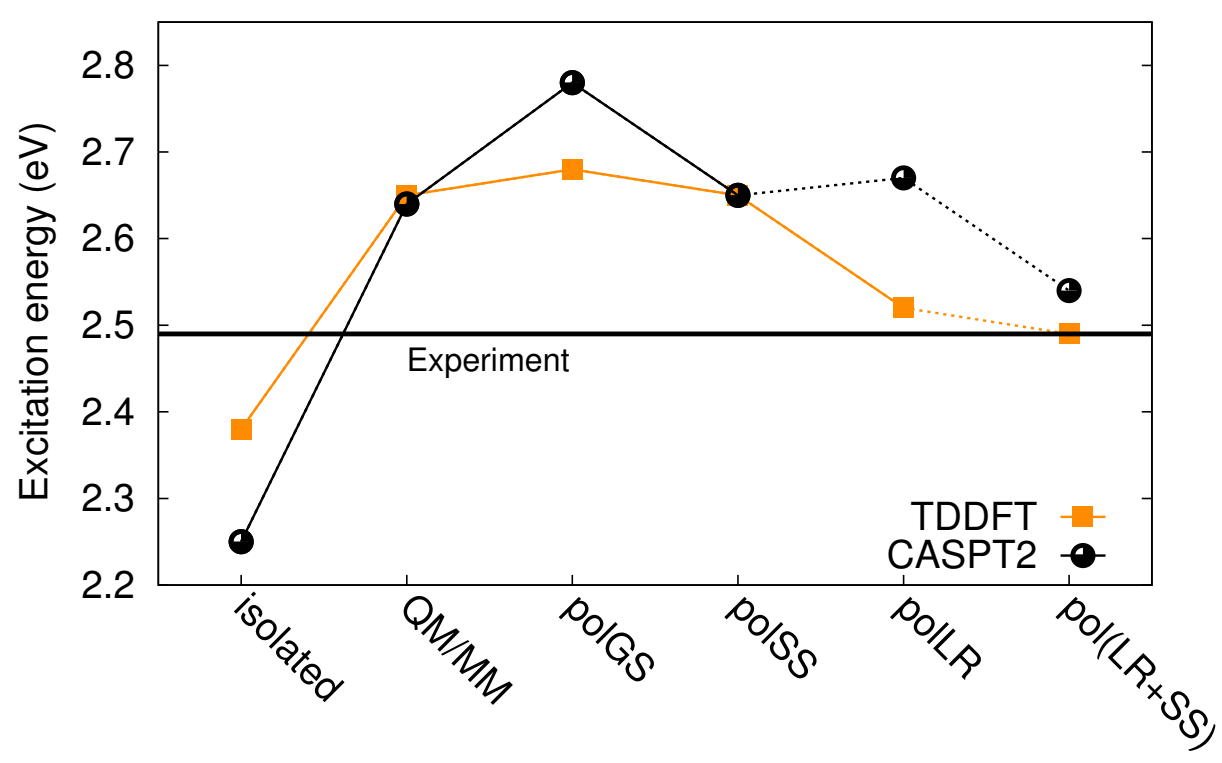

Figure 6.5: Comparison of the TDDFT/CAM-B3LYP and CASPT2 excitation energies $(\mathrm{eV})$ for frame 4 computed for the isolated chromophore and with different models of the protein environment.

validity of our theoretical framework of analysis based on the magnitude of the transition dipole moment the molecular dipole difference, and the resulting polarization effects.

Within CASPT2, we do not have direct access to the excitation energies computed within a linear-response formalism but we can estimate them in two ways: We can either follow Eq. 6.1 and rescale the CASPT2 polSS corrections with the ratio squared of the CASSCF transition dipole moment and the variation of molecular dipoles, or rescale the TDDFT linear-response shifts by the ratio squared of the CASPT2 and TDDFT transition dipole moments,

$$
\Delta \mathrm{E}_{\mathrm{exc} \text { PT2 }}^{(\mathrm{LR}-\mathrm{GS})} \approx \Delta \mathrm{E}_{\mathrm{exc} \text { TDDFT }}^{(\mathrm{LR}-\mathrm{GS})} \frac{\left|\boldsymbol{\mu}_{01 \mathrm{CASSCF}}\right|^{2}}{\left|\boldsymbol{\mu}_{01 \mathrm{TDDFT}}\right|^{2}}
$$

The two estimates are remarkably close and in very good agreement with the corresponding TDDFT linear-response corrections as shown for the three frames in Table 6.2, where the CASPT2 linear-response-like contributions consistently obtained as in Eq. 6.3 are listed.

The behavior of the CASPT2 excitation energies in the different models for the protein environment is also illustrated in Fig. 6.6. For the three snapshots, if we add the state-specific and the estimated linear-response corrections to the polGS values in analogy to what we have done at the TDDFT level, we obtain extrapolated CASPT2 excitation energies in good agreement with the experimental absorption maximum. Also here as discussed in the TDDFT case, the caveat holds that the use of geometries with a larger bond-length alternation than the one obtained with the generalized 


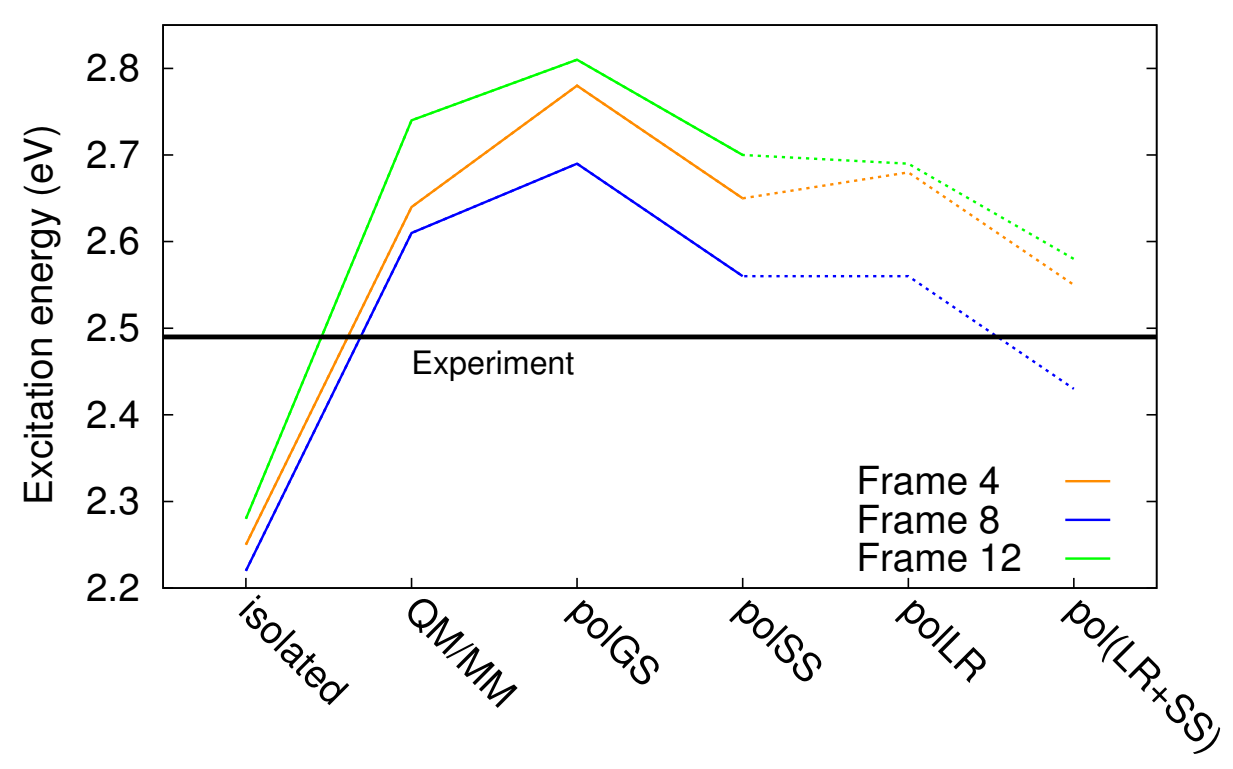

Figure 6.6: Excitation energies $(\mathrm{eV})$ of rhodopsin computed with CASPT2 for RPSB isolated and with different models of the protein environment.

gradient approximation PBE used here would lead to a blue-shift, which is consistent, however, with the vertical excitation energy being higher than the absorption maximum due to the neglect of vibronic effects.

\subsection{Discussion and conclusions}

The analysis carried out within TDDFT and CASPT2 reveals a qualitatively similar behavior between the two approaches to treat the quantum RPSB chromophore coupled to different models for rhodopsin. Furthermore, the quantitative differences can be rationalized (and remarkably well corrected) if one accounts for the reduced electrostatic response of TDDFT in the excited state. This congruence allows us to draw a series of rather robust conclusions on the origin of the chromophore-protein coupling in rhodopsin and on its most suitable theoretical treatment. First, we can argue that an embedding scheme based on point charges is too simplistic to capture the complexity of effects which govern absorption of retinal in rhodopsin and the use of different polarizable models has here given us the needed clues on where a point-charge model goes amiss.

The first consideration is that the use of ground-state-polarized dipoles does not ameliorate the point-charge results but further increases the excitation energies. An improved electrostatic description of ground-state rhodopsin alone is therefore not sufficient and the lack of environmental response to the electronic transition lies at the heart of the problem. The correction due to the electrostatic relaxation of the protein is in fact expected to be large due to the density difference upon excitation 


\begin{tabular}{|c|c|c|c|c|c|c|}
\hline \multirow[t]{2}{*}{ Frame } & \multicolumn{3}{|c|}{ QM/MM } & \multicolumn{3}{|c|}{ QM/MMpol } \\
\hline & $\mathrm{E}_{\mathrm{exc}}$ & $\left|\boldsymbol{\mu}_{01}\right|$ & $|\Delta \mu|$ & $\mathrm{E}_{\text {exc }}^{\text {polGS }}$ & $\Delta \mathrm{E}_{\mathrm{exc}}^{(\mathrm{SS}-\mathrm{GS})}$ & $\Delta \mathrm{E}_{\mathrm{exc}}^{(\mathrm{LR}-\mathrm{GS})}$ \\
\hline & & & & TDDFT & & \\
\hline 4 & 2.652 & 11.86 & 6.89 & 2.680 & -0.034 & -0.160 \\
\hline 8 & 2.630 & 11.87 & 4.71 & 2.627 & -0.018 & -0.158 \\
\hline 12 & 2.651 & 11.83 & 6.18 & 2.662 & -0.028 & -0.160 \\
\hline & & & & CASPT2 & & \\
\hline 4 & 2.645 & 10.39 & 14.75 & 2.784 & -0.136 & -0.100 \\
\hline 8 & 2.609 & 11.02 & 12.38 & 2.686 & -0.129 & -0.127 \\
\hline 12 & 2.724 & 10.54 & 13.55 & 2.809 & -0.113 & -0.119 \\
\hline
\end{tabular}

Table 6.2: Excitation energies (eV) of rhodopsin computed with static QM/MM and the polGS scheme using TDDFT/CAM-B3LYP and CASPT2/ANO-L-VDZP. The polSS and polLR corrections to the polGS excitation energies are listed. The polLR values are computed in linear response at the TDDFT level and estimated in CASPT2 by rescaling the CASPT2 polSS corrections according to Eq. 6.1. The QM/MM transition dipole moment, $\left|\boldsymbol{\mu}_{01}\right|$, and the variation of the molecular dipole moment, $|\Delta \boldsymbol{\mu}|$, are given in Debye.

resulting from the flux of electronic charge from the $\beta$-ionone ring to the nitrogen terminus of RPSB. When this physical feature is properly reproduced by the quantum method chosen to describe the chromophore as in the case of CASPT2, the statespecific correction gives indeed a sizable red-shift of more than $0.1 \mathrm{eV}$, which can be ascribed to the response of several residues in the protein pocket of the chromophore. Nevertheless, the inclusion of electrostatic differential polarization effects does not account for the complete description of the choromophore-protein coupling. The same residues which couple electrostatically to the excitation of the chromophore also resonate to its electronic transition density when treated within a linear-response scheme at the TDDFT level. The resulting correction to the excitation energies is of the same order of magnitude as the electrostatic state-specific one if we account for the relative size of the transition dipole moment and the molecular dipole difference between the ground and the excited state. Their added contribution to the excitation energies results in a good agreement with absorption experiments.

Our analysis demonstrates that the protein-chromophore interactions in rhodopsin are not only of electrostatic nature and are therefore not straightforwardly amenable to a description in terms of a multiscale quantum-in-classical or quantum-in-quantum approach based on the product state of chromophore and protein wave functions. In rhodopsin and any other photobiological system, the process of light absorption is however modulated by the concurrent presence of electrostatic and resonance effects. A computational option to recover them at once is to perform an explicit quantum calculation on an extended region surrounding the photoactive moiety, employing a technique able to describe coupled excitations between the chromophore and the many residues we have shown to participate in the excitation process. This is unfortunately very expensive and currently limited to the use of low-correlation approaches. 
Here, we have however outlined an alternative, practical route where a classical embedding model is adapted to mimic both electrostatic and resonant coupling, and to provide key information on the origin of the interactions and the detailed quantitative role of specific amino acids in the photoexcitation of a complex chromophore-protein system.

\subsection{Computational methods}

Our model system consists of two rhodopsin monomers embedded in an explicit phospholipidic membrane. All details about the construction of the model are given in Ref. [4].

We use the MOLCAS 7.8 suite of programs [42] for the CASPT2 [43,44] calculations always employing the recommended zero-order Hamiltonian [45] with the IPEA shift set to 0.25 a.u. To prevent the occurrence of intruder states in the perturbation treatment, we use an additional constant imaginary shift [46] of 0.1 a.u. Unless otherwise specified, the values of the excitation energies reported are computed at the single-state level. The zero-order wave function is a state-average SA-CASSCF wave function with an active space CAS $(12,13)$ correlating 12 electrons in $13 \pi$ orbitals. Two roots with equal weights are used in the calculation. For all CASSCF and CASPT2 calculations, we employ the ANO-L-VDZP basis set [47].

The CASPT2/MMpol results are computed in a two-step procedure [48]: The $\mathrm{MM}$ induced dipoles and charges from a CASSCF/MMpol calculation with the Gaussian code [49] are later used in MOLCAS as a static external potential to obtain the CASPT2 values. We perform the CASSCF/MMpol calculations with a locally modified version of Gaussian09, revision A.02. Also these calculations are performed in a state-average fashion as with the same parameters described above, and we adapt the MM dipoles either to the ground state (polGS) or to the ground and excited states in two separate calculations (polSS).

The TDDFT excitation energies are computed using a locally modified version of Gaussian09, revision A.02 [49] and the CAM-B3LYP [50] exchange-correlation functional in combination with the $6-31+\mathrm{G}^{*}$ basis set. We employ the MMpol embedding in a limited region of the protein environment, which includes the residues placed within $15 \AA$ of the chromophore, while the rest of the protein is described with static point charges only. This polarization threshold has been shown to provide converged values of the excitation energies on previous studies on rhodopsin [51,52]. In all the calculations the boundary of the quantum region is placed at the $\mathrm{C}_{\gamma}-\mathrm{C}_{\delta}$ bond of the Lys296 residue covalently linked to the retinal moiety, creating the retinal protonated Schiff base (see Fig. 6.1).

In the QM/MMpol calculations, we use the Amber pol12 parameters (AL model in Ref. [53,54]) to describe the protein and the water solvent. For water, we derived the point charges from a standard RESP fit using the electrostatic potential computed at the MP2/aug-cc-pVTZ level on the TIP3P water geometry $\left(\mathrm{q}_{\mathrm{O}}=-0.726, \mathrm{q}_{\mathrm{H}}=\right.$ 0.363). For the residues further than $15 \AA$ from the chromophore, we use the static Amber 03 force field. 


\section{Bibliography}

[1] T. Ebrey and Y. Koutalos, Prog. Retinal and Eye Res. 20, 49 (2001).

[2] B. Nickle and P. R. Robinson, Cell. Mol. Life. Sci. 64, 2917 (2007).

[3] O. P. Ernst, D. T. Lodowski, M. Elstner, P. Hegemann, L. S. Brown, and H. Kandori, Chem. Rev. 114, 126 (2014).

[4] O. Valsson, P. Campomanes, I. Tavernelli, U. Rothlisberger, and C. Filippi, J. Chem. Theory Comput. 9, 2441 (2013).

[5] C.-M. Suomivuori, L. Lang, D. Sundholm, A. P. Gamiz-Hernandez, and V. R. I. Kaila, Chem. Eur. J. 22, 8254 (2016).

[6] M. Wanko, M. Hoffmann, T. Frauenheim, and M. Elstner, J. Phys. Chem. B 112, 11462 (2008).

[7] M. Wanko, M. Hoffmann, J. Frähmcke, T. Frauenheim, and M. Elstner, J. Phys. Chem. B 112, 11468 (2008).

[8] K. Katayama, S. Sekharan, and Y. Sudo, in Optogenetics: Light-Sensing Proteins and Their Applications, edited by H. Yawo, H. Kandori, and A. Koizumi (Springer Japan, 2015), Chap. Color Tuning in Retinylidene Proteins, pp. 89107.

[9] K. Palczewski, Annu. Rev. Biochem 75, 743 (2006).

[10] K. Palczewski, T. Kumasaka, T. Hori, C. A. Behnke, H. Motoshima, B. A. Fox, I. L. Trong, D. C. Teller, T. Okada, R. E. Stenkamp, M. Yamamoto, and M. Miyano, Science 289, 739 (2000).

[11] E. C. Y. Yan, M. A. Kazmi, S. De, B. S. W. Chang, C. Seibert, E. P. Marin, R. A. Mathies, and T. P. Sakmar, Biochemistry 41, 3620 (2002).

[12] H. Houjou, Y. Inoue, and M. Sakurai, J. Phys. Chem. B 105, 867 (2001).

[13] N. Ferré and M. Olivucci, J. Am. Chem. Soc. 125, 6868 (2003).

[14] M. Wanko, M. Hoffmann, P. Strodel, A. Koslowski, W. Thiel, F. Neese, T. Frauenheim, and M. Elstner, J. Phys. Chem. B 109, 3606 (2005). 
[15] U. F. Röhrig, L. Guidoni, and U. Rothlisberger, ChemPhysChem 6, 1836 (2005).

[16] P. B. Coto, A. Strambi, N. Ferré, and M. Olivucci, Proc. Natl. Acad. Sci. U.S.A. 103, 17154 (2006).

[17] A. Matsuura, H. Sato, H. Houjou, S. Saito, T. Hayashi, and M. Sakurai, J. Comput. Chem. 27, 1623 (2006).

[18] M. Hoffmann, M. Wanko, P. Strodel, P. H. König, T. Frauenheim, K. Schulten, W. Thiel, E. Tajkhorshid, and M. Elstner, J. Am. Chem. Soc. 128, 10808 (2006).

[19] K. Fujimoto, S. Hayashi, J. ya Hasegawa, and H. Nakatsuji, J. Chem. Theory Comput. 3, 605 (2007).

[20] K. Bravaya, A. Bochenkova, A. Granovsky, and A. Nemukhin†, J. Am. Chem. Soc. 129, 13035 (2007).

[21] A. Strambi, P. B. Coto, N. Ferré, and M. Olivucci, Theor. Chem. Acc. 118, 185 (2007).

[22] A. Altun, S. Yokoyama, and K. Morokuma, J. Phys. Chem. B 112, 16883 (2008).

[23] A. Altun, S. Yokoyama, and K. Morokuma, J. Phys. Chem. B 112, 6814 (2008).

[24] K. Fujimoto, J. Hasegawa, and H. Nakatsuji, Bull. Chem. Soc. Jpn 82, 1140 (2009).

[25] T. Andruniów and M. Olivucci, J. Chem. Theory Comput. 5, 3096 (2009).

[26] G. Tomasello, G. Olaso-González, P. Altoè, M. Stenta, L. Serrano-Andrés, M. Merchán, G. Orlandi, A. Bottoni, and M. Garavelli, J. Am. Chem. Soc. 131, 5172 (2009).

[27] I. V. Rostov, R. D. Amos, R. Kobayashi, G. Scalmani, and M. J. Frisch, J. Phys. Chem. B 114, 5547 (2010).

[28] J. Hasegawa, K. J. Fujimoto, and H. Nakatsuji, ChemPhysChem 12, 3106 (2011).

[29] F. Melaccio, M. Olivucci, R. Lindh, and N. Ferré, Int. J. Quantum Chem. 111, 3339 (2011).

[30] S. Sekharan and K. Morokuma, J. Am. Chem. Soc. 133, 19052 (2011).

[31] P. Campomanes, M. Neri, B. A. C. Horta, U. F. Röhrig, S. Vanni, I. Tavernelli, and U. Rothlisberger, J. Am. Chem. Soc. 136, 3842 (2014).

[32] X. Zhou, D. Sundholm, T. A. Wesołowski, and V. R. I. Kaila, J. Am. Chem. Soc. 136, 2723 (2014). 
[33] E. M. Sproviero, J. Chem. Theory and Comput. 11, 1206 (2015).

[34] T. Yanai, D. P. Tew, and N. C. Handy, Chem. Phys. Lett. 393, 51 (2004).

[35] S. Corni, R. Cammi, B. Mennucci, and J. Tomasi, J. Chem. Phys. 123, 134512 (2005).

[36] J. P. Perdew, K. Burke, and M. Ernzerhof, Phys. Rev. Letters 77, 3865 (1996).

[37] R. Cammi, S. Corni, B. Mennucci, and J. Tomasi, J. Chem. Phys. 122, 104513 (2005).

[38] R. Mathies and L. Stryer, Proc. Natl Acad. Sci. USA 73, 2169 (1976).

[39] M. Ponder and R. Mathies, J. Phys. Chem. 87, 5090 (1983).

[40] J. Bednarska, A. Roztoczyńska, W. Bartkowiak, and R. Zaleśny, Chem. Phys. Lett. 584, 58 (2013).

[41] M. Caricato, G. W. Trucks, M. J. Frisch, and K. B. Wiberg, J. Chem. Theory Comput. 7, 456 (2011).

[42] F. Aquilante, L. De Vico, N. Ferré, G. Ghigo, P.-å. Malmqvist, P. Neogrády, T. B. Pedersen, M. Pitoňák, M. Reiher, B. O. Roos, L. Serrano-Andrés, M. Urban, V. Veryazov, and R. Lindh, J. Comput. Chem. 31, 224 (2010).

[43] K. Andersson, P.-A. Malmqvist, B. O. Roos, A. J. Sadlej, and K. Wolinski, J. Phys. Chem. 94, 5483 (1990).

[44] K. Andersson, P.-A. Malmqvist, and B. O. Roos, J. Chem. Phys. 96, 1218 (1992).

[45] G. Ghigo, B. O. Roos, and P.-Å. Malmqvist, Chem. Phys. Lett. 396, 142 (2004).

[46] N. Forsberg and P.-Å. Malmqvist, Chem. Phys. Lett. 274, 196 (1997).

[47] P. Widmark, P. Malmqvist, and B. O. Roos, Theor. Chem. Acc. 77, 291 (1990).

[48] Q. Li, B. Mennucci, M. A. Robb, L. Blancafort, and C. Curutchet, J. Chem. Theory Comput. 11, 1674 (2015).

[49] M. J. Frisch, G. W. Trucks, H. B. Schlegel, G. E. Scuseria, M. A. Robb, J. R. Cheeseman, G. Scalmani, V. Barone, B. Mennucci, G. A. Petersson, H. Nakatsuji, M. Caricato, X. Li, H. P. Hratchian, A. F. Izmaylov, J. Bloino, G. Zheng, J. L. Sonnenberg, M. Hada, M. Ehara, K. Toyota, R. Fukuda, J. Hasegawa, M. Ishida, T. Nakajima, Y. Honda, O. Kitao, H. Nakai, T. Vreven, J. A. Montgomery, Jr., J. E. Peralta, F. Ogliaro, M. Bearpark, J. J. Heyd, E. Brothers, K. N. Kudin, V. N. Staroverov, R. Kobayashi, J. Normand, K. Raghavachari, A. Rendell, J. C. Burant, S. S. Iyengar, J. Tomasi, M. Cossi, N. Rega, J. M. Millam, 
M. Klene, J. E. Knox, J. B. Cross, V. Bakken, C. Adamo, J. Jaramillo, R. Gomperts, R. E. Stratmann, O. Yazyev, A. J. Austin, R. Cammi, C. Pomelli, J. W. Ochterski, R. L. Martin, K. Morokuma, V. G. Zakrzewski, G. A. Voth, P. Salvador, J. J. Dannenberg, S. Dapprich, A. D. Daniels, Ö. Farkas, J. B. Foresman, J. V. Ortiz, J. Cioslowski, and D. J. Fox, Gaussian 09 Revision A.02, Gaussian Inc. Wallingford CT 2009.

[50] T. Yanai, D. P. Tew, and N. C. Handy, Chem. Phys. Lett. 393, 51 (2004).

[51] P. Söderhjelm, C. Husberg, A. Strambi, M. Olivucci, and U. Ryde, J. Chem. Theory Comput. 5, 649 (2009).

[52] M. T. Beerepoot, A. H. Steindal, K. Ruud, J. M. H. Olsen, and J. Kongsted, Comput. Theor. Chem. 1040, 304 (2014).

[53] J. Wang, P. Cieplak, J. Li, T. Hou, L. Ray, and D. Yong, J. Chem. Phys. B 8, 3091 (2011).

[54] J. Wang, P. Cieplak, J. Li, J. Wang, Q. Cai, M. Hsieh, H. Lei, R. Luo, and Y. Duan, J. Chem. Phys. B 8, 3100 (2011). 


\section{List of publications}

- R. Guareschi, and C. Filippi,

"Ground- and excited-state geometry optimization of small organic molecules with quantum Monte Carlo"

J. Chem. Theory Comput. 9, 5513 (2013). (Chapter 3)

- R. Guareschi, F. M. Floris, C. Amovilli, and C. Filippi

"Solvent effects on excited-state structures: A quantum Monte Carlo and density functional study"

J. Chem. Theory Comput. 10, 5528 (2014). (Chapter 4)

- R. Guareschi, H. Zulfikri, C. Daday, F. M. Floris, C. Amovilli, B. Mennucci, and C. Filippi,

"Introducing QMC/MMpol: Quantum Monte Carlo in polarizable force fields for excited states"

J. Chem. Theory Comput. 12, 1674 (2016). (Chapter 5)

- R. Guareschi, O. Valsson, C. E. Curutchet, B. Mennucci, and C. Filippi, "Dissecting polarization in photoexcited rhodopsin: Quantum effects from classical modeling"

In preparation (2016). (Chapter 6) 


\section{Samenvatting}

De ontwikkeling van theoretische methoden in de kwantumchemie heeft zich lange tijd beperkt tot de beschrijving van grondtoestand eigenschappen, en pas sinds kort de aandacht verlegd naar de studie van aangeslagen toestanden. In het algemeen is het moeilijk betrouwbare berekenen uit te voeren van excitatie eigenschappen, en de methoden die gebruikt worden om foto-actieve moleculen te bestuderen gaan gepaard met grote problemen, vooral als men zich ver buiten het Franck-Condon gebied begeeft en de invloed van omringende moleculen in rekening wil brengen. In dit proefschrift zullen we nieuwe theoretische methoden ontwikkelen en diepgaand inzicht bieden in de simulatie van structurele relaxaties van moleculen in een aangeslagen toestand in gecondenseerde fasen, en in de beschrijving van de koppeling van de actieve spot met zijn omgeving bij de berekening van absorptie energieën van een realistisch foto-biologisch systeem.

In Hoofdstuk 3, onderzoeken we de geometrie optimalisatie in de aangeslagen toestand van kleine prototypische organische moleculen in de gasfase met behulp van kwantum Monte Carlo (QMC) methoden. Voor alle moleculen levert QMC in zijn eenvoudigste variationele variant geometrieën in zeer goede overeenstemming met storings-theoretische resultaten, terwijl tegelijkertijd de methode veel robuuster is met betrekking tot de rekenkundige parameters die bij de berekening gebruikt worden. Dit staat in contrast met de grote variatie in structuren verkregen met de rekenkundig goedkopere tijdsafhankelijke dichtheidsfunctionaal theorie (TDDFT) wanneer gebruik wordt gemaakt van verschillende exchange-correlatie functionalen, terwijl er geen duidelijke relatie bestaat tussen de gekozen klasse van functionalen en de kwaliteit van de corresponderende structuren. Onze bevindingen tonen de goede prestaties van QMC ook in gevallen waarin andere gecorreleerde of TDDFT benaderingen onnauwkeurig zijn, en tonen aan dat QMC een krachtige methode is die als referentie kan dienen voor toekomstige structurele studies, ook van grotere systemen.

Gestimuleerd door de resultaten van hoofdstuk 3, combineren we nu, in Hoofdstuk 4, voor de eerste keer QMC, in zijn variationele variant, met het polariseerbare continuüm model (PCM) om geometrie optimalisatie van de aangeslagen toestand uit te voeren voor moleculen in oplossing. In onze aanpak wordt het externe veld ten gevolge van het continuüm medium, dat hier een wateroplossing representeert, tijdens de QMC optimalisatie van de geometrie van het opgeloste molecuul, consistent met de moleculaire golffunctie gehouden. Deze methode wordt hier gebruikt om de kwaliteit van de respons van structuren in de aangeslagen toestand op de aan- 
wezigheid van een omgeving, zoals die berekend wordt met TDDFT, te beoordelen. We tonen aan dat alle onderzochte TDDFT functionalen de geometrische respons in de aangeslagen toestand op de aanwezigheid van een oplosmiddel overschatten, soms zelfs bond variaties laten zien met trends tegenovergesteld aan die met QMC worden gevonden.

In Hoofdstuk 5 verlaten wij het onderwerp van de geometrie optimalisatie en richten wij ons op het modelleren van absorptie-eigenschappen met gebruikmaking van een meer realistische, discrete weergave van de responsieve omgeving. Wij introduceren een kwantum-in-klassieke multiscale schema dat kwantum Monte Carlo combineert met een reactie veld van klassieke, polariseerbare dipolen (QMC / MMpol). We onderzoeken de kwaliteit van dit multiscale model voor het beschrijven van verticale excitatie energieën van prototypische kleine gesolvateerde moleculen. De vergelijking met super-moleculaire berekeningen, waarin zowel de opgeloste stof als het oplosmiddel kwantum mechanisch worden behandeld, laat zien dat het gebruik van sets van dipolen, die afzonderlijk in evenwicht gebracht worden met de twee toestanden die betrokken zijn bij de elektronische transitie, leidt tot betere resultaten dan het gebruik van een bevroren omgeving (puntladingen of dipolen gepolariseerd door de opgeloste stof in zijn grondtoestand), in het bijzonder wanneer de chromofoor zeer verschillende moleculaire dipolen heeft in de grondtoestand en de aangeslagen toestand. QMC / MMpol geldt daarom als een zeer effectieve methode voor de behandeling van belangrijke elektrostatische omgevingseffecten voorbij statische puntladingen, die de nauwkeurigheid van QMC combineert met de eenvoud van een klassieke benadering.

In Hoofdstuk 6 richten wij ons op het moeilijke geval van lichtabsorptie van de 11- cis retinaal geprotoneerde Schiff base (RPSB) chromofoor in rhodopsine, die sterk wordt gemoduleerd door interacties met de aminozuren van het bindende pocket van het eiwit. Met behulp van het superieure instrument van polariseerbare krachtvelden, willen we de aard van deze interacties verhelderen, en begrijpen of men verder moet gaan dan een elektrostatische beschrijving, en subtielere kwantumeffecten moet inbouwen. Voor een reeks van frames gekozen uit een kwantumin-klassieke moleculaire dynamica, berekenen we de excitatie energieën in een veld van dipolen in evenwicht met de grondtoestand en die in een veld van toestandsspecifieke dipolen. Daarnaast voeren wij berekeningen uit waarin wij, met behulp van lineaire-response theorie, klassieke dipolen induceren als reactie op de RPSB overgangsdichtheid geassocieerd met de elektronische overgang. In overeenstemming met het grote verschil in elektronen dichtheden tussen de grond en aangeslagen toestand, verbeteren differentiële polarisatie-effecten berekend met behulp van toestand-specifieke dipolen de overeenkomst met absorptie experimenten vergeleken met die berekend met punt ladingen aanzienlijk, maar zijn zij niet in staat een volledige beschrijving te geven van de chromofoor-eiwit koppeling. Het hier voorgestelde gebruik van dipolen verkregen in resonantie met de chromofoor overgang heeft ook een grote invloed op de excitatie, wat aangeeft dat niet-klassieke, resonante interacties tussen RPSB en verschillende omringende aminozuren belangrijk zijn. Wanneer dit effect in rekening wordt gebracht tezamen met de elektrostatische reactie van de omgeving op de excitatie, krijgen we excitatie energieën in goede overeenstemming 
met het experimentele absorptie maximum van rhodopsine. 


\section{Acknowledgments}

First and foremost, I want to thank my promoter, Prof. Claudia Filippi, for her precious work done with me during the years of this Ph.D. I am particularly grateful not only for her undisputed scientific strength, but also for the daily example of dedication and passion for this work that she has always showed. Needless to say, none of this would have been possible without her constant careful supervision.

In the same way, I want to express my gratitude to Prof. Claudio Amovilli and Dr. Franca Maria Floris for welcoming me twice as visiting Ph.D. student in their group at the University of Pisa. During the weeks spent with them, I had the chance to learn about the polarizable continuum model and also a first approach to the use of polarizable force fields.

This topic was then thoroughly investigated during another visit at the same University, within the group of Prof. Benedetta Mennucci. For this reason, I want to thank her and all her group for their teachings and valuable help.

Among the people met in my daily university life, I want to mention my previous colleague Csaba, who gave me a big help during my first months and always productively collaborated with me. I want also to thank Omar, for our recent work on the study of rhodopsin and for his careful scientific support. I wish all the best to my colleagues and friends who are currently walking their way through the Ph.D. studies, Habib, Monika, Matteo, and The Vishals. Together with the Professors Wim Briels and Wouter den Otter, I cannot forget to mention Ioana, who recently earned her Ph.D. and has been a true enthusiastic leader in the organization of group activities and outings during these years. I also want to thank Barry for our sport-related conversations during lunch and coffee breaks which gave me the chance to learn a lot also in extra-scientific fields. Thanks also to the secretary of our group, Els, for her fundamental help with all the paperwork and documents needed during these years.

Outside work, I had the chance to come in touch with many people during these years, who have been an important part of my Ph.D. experience. First of all, I want to remember and acknowledge the large Italian community I met here in Enschede, for all the good moments we shared: Virginia, Giorgio, Matteo, Federico, and Caterina, who have been a constant presence during my four years here, and also Tommaso, Loredana, Alberto, and Ludovica, who joined more recently. A special mention goes of course to the Sicilians, Marco, the first friend that I met in the Netherlands, Giuseppe, who came to stay a few months and ended up spending almost two years in Enschede, making them for sure more enjoyable, and Giorgio for his dialect lessons. I also want to remember Alessia who has become a sincere friend and to whom I wish 
all the best for her studies in Utrecht. Finally, thanks to Riccardo for the evenings spent commenting football games and Roberto for our swimming sessions.

Beside them, my acknowledgments go to Alex, Xavier, and Reshi for our basketball afternoons and for the several tips that I received to improve my playing skills. Hard work pays off, in work as much as in sports.

Obviously, I cannot forget the Greeks, Vasilis, Dimitris (all of them), Stergios, Kostas, and ... of course Katerina. To her, in particular, goes my gratitude for always showing patience and support, for the many sacrifices she made to maximize the time spent together every weekend, and for her constant pure enthusiasm that makes every day special.

In the last four years, I surely met many new people, but I never forgot the old friends. Thanks to Mattia and Nadine for always being at my side to give me advice and assistance and for all the good holidays together. Those moments and memories are precious to me and will not be forgotten. Most of all, than you for cheering me up when I was feeling down and for making me feel even better in my good days.

Although I am not a nostalgic person, being in touch with my pals from Rimini has been important during all this time abroad and for this reason my thoughts go to Alessandro, Matteo, Giagio-M , Giacomo, Kappa, Luca, Zazzo, Nick, Casa, Mazza, Giordano, Cicca, Lorenzo, and Scarmo. Thank you guys, for all the chats and fun.

Last but not least, I have to express my fullest thankfulness to my parents, for always giving me everything I needed, for my education, for leaving me the total freedom of my choices and for bearing my absence for large part of the year. You really make me feel blessed. 
